\title{
BI-PARAMETER TRILINEAR FOURIER MULTIPLIERS AND PSEUDO-DIFFERENTIAL OPERATORS WITH FLAG SYMBOLS
}

\author{
GUOZHEN LU, JILL PIPHER, AND LU ZHANG
}

\begin{abstract}
The main purpose of this paper is to study $L^{r}$ Hölder type estimates for a bi-parameter trilinear Fourier multiplier with flag singularity, and the analogous pseudo-differential operator, when the symbols are in a certain product form. More precisely, for $f, g, h \in \mathcal{S}\left(\mathbb{R}^{2}\right)$, the bi-parameter trilinear flag Fourier multiplier operators we consider are defined by
\end{abstract}

$$
T_{m_{1}, m_{2}}(f, g, h)(x):=\int_{\mathbb{R}^{6}} m_{1}(\xi, \eta, \zeta) m_{2}(\eta, \zeta) \hat{f}(\xi) \hat{g}(\eta) \hat{h}(\zeta) e^{2 \pi i(\xi+\eta+\zeta) \cdot x} d \xi d \eta d \zeta,
$$

when $m_{1}, m_{2}$ are two bi-parameter symbols. We study Hölder type estimates: $L^{p_{1}} \times L^{p_{2}} \times L^{p_{3}} \rightarrow L^{r}$ for $1<p_{1}, p_{2}, p_{3}<\infty$ with $1 / p_{1}+1 / p_{2}+1 / p_{3}=1 / r$, and $0<r<\infty$. We will show that our problem can be reduced to establish the $L^{r}$ estimate for the special multiplier $m_{1}\left(\xi_{1}, \eta_{1}, \zeta_{1}\right) m_{2}\left(\eta_{2}, \zeta_{2}\right)$ (see Theorem 1.7).

We also study these $L^{r}$ estimates for the corresponding bi-parameter trilinear pseudo-differential operators defined by

$$
T_{a b}(f, g, h)(x):=\int_{\mathbb{R}^{6}} a(x, \xi, \eta, \zeta) b(x, \eta, \zeta) \hat{f}(\xi) \hat{g}(\eta) \hat{h}(\zeta) e^{2 \pi i x(\xi+\eta+\zeta)} d \xi d \eta d \zeta,
$$

where the smooth symbols $a, b$ satisfy certain bi-parameter Hörmander conditions. We will also show that the $L^{r}$ estimate holds for $T_{a b}$ as long as the $L^{r}$ estimate for the flag multiplier operator holds when the multiplier has the special form $m_{1}\left(\xi_{1}, \eta_{1}, \zeta_{1}\right) m_{2}\left(\eta_{2}, \zeta_{2}\right)$ (see Theorem 1.10). Using our reduction of the flag multiplier, we also provide an alternative proof of some of the mixed norm estimates recently established by Muscalu and Zhai [23] when the functions $g$ and $h$ are of tensor product forms (Theorem 1.8). Moreover, our method also allow us to establish the weighted mixed norm estimates (Theorem 1.9).

The bi-parameter and trilinear flag Fourier multipliers considered in this paper do not satisfy the conditions of the classical bi-parameter trilinear Fourier multipliers considered by Muscalu, Tao, Thiele and the second author 21,22. They may also be viewed as the bi-parameter trilinear variants of estimates obtained for the one-parameter flag paraproducts by Muscalu [18.

Key words and phrases. Bi-parameter, trilinear operator, flag paraproducts, Fourier multipliers, pseudo-differential operators.

The first author's research was partly supported by a collaboration grant from the Simons Foundation. 


\section{INTRODUCTION}

For $n \geq 1$ we denote by $\mathcal{M}\left(\mathbb{R}^{n}\right)$ the set of all bounded symbols $m \in L^{\infty}\left(\mathbb{R}^{n}\right)$, smooth away from the origin and satisfying the classical Marcinkiewcz-MikhlinHörmander condition

$$
\left|\partial^{\alpha} m(\xi)\right| \lesssim \frac{1}{|\xi|^{\alpha}}
$$

for every $\xi \in \mathbb{R}^{n} \backslash\{0\}$ and sufficiently many multi-indices $\alpha$. Denote by $T_{m}$ the n-linear operator

$$
T_{m}\left(f_{1}, \ldots, f_{n}\right)(x):=\int_{\mathbb{R}^{n}} m(\xi) \hat{f}_{1}\left(\xi_{1}\right) \cdots \hat{f}_{n}\left(\xi_{n}\right) e^{2 \pi i\left(\xi_{1}+\cdots+\xi_{n}\right) \cdot x} d \xi,
$$

where $\xi=\left(\xi_{1}, \ldots, \xi_{n}\right) \in \mathbb{R}^{n}$ and $f_{1}, \ldots, f_{n}$ are Schwartz functions on $\mathbb{R}$, denoted by $\mathcal{S}(\mathbb{R})$. From the classical Coifman-Meyer theorem we know $T$ extends to a bounded n-linear operator from $L^{p_{1}}(\mathbb{R}) \times \cdots \times L^{p_{n}}(\mathbb{R})$ to $L^{r}(\mathbb{R})$ for $1<p_{1}, \ldots, p_{n} \leq \infty$ and $1 / p_{1}+\cdots+1 / p_{n}=1 / r>0$. In fact this property holds in higher dimensions when $f_{i} \in L^{p_{i}}\left(\mathbb{R}^{d}\right), i=1, \ldots, n$ and $m \in \mathcal{M}\left(\mathbb{R}^{n d}\right)$, see [5, 13, 14. The case $p \geq 1$ was proved by Coifman and Meyer [5] and was extended to $p<1$ by Grafakos and Torres [13, and Kenig and Stein [14.

For the corresponding pseudo-differential variant of the classical Coifman-Meyer theorem, suppose that the symbol $\sigma(x, \xi)$ belongs to the Hörmander symbol class $S_{1,0}^{0}\left(\mathbb{R} \times \mathbb{R}^{n}\right)$; that is, $\sigma$ satisfies the condition

$$
\left|\partial_{x}^{l} \partial_{\xi}^{\alpha} \sigma(x, \xi)\right| \lesssim \frac{1}{(1+|\xi|)^{|\alpha|}}
$$

for any $x \in \mathbb{R}, \xi=\left(\xi_{1}, \ldots, \xi_{n}\right) \in \mathbb{R}^{n}$ and all indices $l, \alpha$. For these symbols, the following multi-linear, single parameter case has been studied.

Theorem 1.1 ( [13, 20]). The operator

$$
T_{\sigma}\left(f_{1}, \ldots, f_{n}\right)(x):=\int_{\mathbb{R}^{n}} \sigma(x, \xi) \hat{f}_{1}\left(\xi_{1}\right) \cdots f_{n}\left(\xi_{n}\right) e^{2 \pi i\left(\xi_{1}+\cdots+\xi_{n}\right) \cdot x} d \xi
$$

is bounded from $L^{p_{1}}(\mathbb{R}) \times \cdots \times L^{p_{n}}(\mathbb{R})$ to $L^{r}(\mathbb{R})$ for $1<p_{1}, \ldots, p_{n} \leq \infty$ and $1 / p_{1}+$ $\cdots+1 / p_{n}=1 / r>0$, where $f_{1}, \ldots, f_{n} \in \mathcal{S}(\mathbb{R})$ and $\sigma$ satisfies (1.3). Again, this result still hold if the functions are defined on $\mathbb{R}^{d}$.

We now consider the multi-parameter setting of the above operators, introduced and studied via time-frequency analysis in [20 22]. For simplicity, we just state the bi-linear, bi-parameter case when $f, g$ are defined on $\mathbb{R}^{2}$. The results extend to the $n$-linear, d-parameter case where $f_{1}, \ldots, f_{n}$ are defined on $\mathbb{R}^{d}$. We denote by $m \in \mathcal{B M}\left(\mathbb{R}^{4}\right)$ the set of smooth bi-parameter symbols satisfying

$$
\left|\partial_{\xi_{1}, \xi_{2}}^{\alpha_{1}, \alpha_{2}} \partial_{\eta_{1}, \eta_{2}}^{\beta_{1}, \beta_{2}} m(\xi, \eta)\right| \lesssim \prod_{i=1}^{2} \frac{1}{\left(\left|\xi_{i}\right|+\left|\eta_{i}\right|\right)^{\alpha_{i}+\beta_{i}}}
$$


for any $\xi=\left(\xi_{1}, \xi_{2}\right), \eta=\left(\eta_{1}, \eta_{2}\right) \in \mathbb{R}^{2} \backslash\{0\}$ and sufficiently many multi-indices $\alpha=\left(\alpha_{1}, \alpha_{2}\right), \beta=\left(\beta_{1}, \beta_{2}\right)$

Theorem $1.2([2022])$. Let $1<p, q \leq \infty, 1 / p+1 / q=1 / r, 0<r<\infty$ and $m \in \mathcal{B} \mathcal{M}\left(\mathbb{R}^{4}\right)$, then the operator

$$
T_{m}(f, g)(x)=\int_{\mathbb{R}^{4}} m\left(\xi_{1}, \xi_{2}, \eta_{1}, \eta_{2}\right) e^{2 \pi i x(\xi+\eta)} \hat{f}\left(\xi_{1}, \xi_{2}\right) \hat{g}\left(\eta_{1}, \eta_{2}\right) d \xi d \eta
$$

is bounded from $L^{p}\left(\mathbb{R}^{2}\right) \times L^{q}\left(\mathbb{R}^{2}\right) \rightarrow L^{r}\left(\mathbb{R}^{2}\right)$.

A Hörmander type multiplier theorem with limited smoothness on the multiparameter and multilinear multipliers was obtained in [3].

Theorem 1.3 ([3]). Let $m \in C^{2 d+1}\left(\mathbb{R}^{2 d} \backslash\{0\} \times \mathbb{R}^{2 d} \backslash\{0\}\right)$ satisfy (1.4) for all $\left|\alpha_{1}\right|+\left|\beta_{1}\right| \leq d+1,\left|\alpha_{2}\right|+\left|\beta_{2}\right| \leq d+1$ and $\left(\xi_{1}, \xi_{2}, \eta_{1}, \eta_{2}\right) \in\left(\mathbb{R}^{2 d} \backslash\{0\} \times \mathbb{R}^{2 d} \backslash\{0\}\right)$. Then $T_{m}$ defined in (1.5) is bounded from $L^{p}\left(\mathbb{R}^{2 d}\right) \times L^{q}\left(\mathbb{R}^{2 d}\right) \rightarrow L^{r}\left(\mathbb{R}^{2 d}\right)$ for $1<p, q<\infty$, $1 / p+1 / q=1 / r, 0<r<\infty$.

The corresponding bi-parameter pseudo-differential operator was studied in [7].

Theorem 1.4 ( [7]). Define

$$
T_{a}(f, g)(x):=\int_{\mathbb{R}^{4}} a(x, \xi, \eta) \hat{f}\left(\xi_{1}, \xi_{2}\right) \hat{g}\left(\eta_{1}, \eta_{2}\right) e^{2 \pi i x \cdot(\xi+\eta)} d \xi d \eta
$$

where

$$
\left|\partial_{x_{1}}^{l_{1}} \partial_{x_{2}}^{l_{2}} \partial_{\xi_{1}}^{\alpha_{1}} \partial_{\xi_{2}}^{\alpha_{2}} \partial_{\eta_{1}}^{\beta_{1}} \partial_{\eta_{2}}^{\beta_{2}} a(x, \xi, \eta)\right| \lesssim \frac{1}{\left(1+\left|\xi_{1}\right|+\left|\eta_{1}\right|\right)^{\alpha_{1}+\beta_{1}}} \frac{1}{\left(1+\left|\xi_{2}\right|+\left|\eta_{2}\right|\right)^{\alpha_{2}+\beta_{2}}}
$$

Then $T_{a}$ is bounded on $L^{p_{1}} \times L^{p_{2}} \rightarrow L^{r}$ provided that $1<p_{1}, p_{2} \leq \infty$ and $\frac{1}{r}=$ $\frac{1}{p_{1}}+\frac{1}{p_{2}}>0$.

In particular, in the proof of trilinear bi-parameter version of Theorem 1.4 above, the following localized $L^{r}$ estimates hold and these estimates will also play a role in our current paper.

Theorem 1.5 ( [7]). Let $m(\xi, \eta, \zeta)$ be a smooth symbol satisfying

$$
\left|\partial_{\xi_{1}, \xi_{2}}^{\alpha_{1}, \alpha_{2}} \partial_{\eta_{1}, \eta_{2}}^{\beta_{1}, \beta_{2}} \partial_{\zeta_{1}, \zeta_{2}}^{\gamma_{1}, \gamma_{2}} m(\xi, \eta, \zeta)\right| \lesssim \prod_{i=1}^{2} \frac{1}{\left(1+\left|\xi_{i}\right|+\left|\eta_{i}\right|+\left|\zeta_{i}\right|\right)^{\alpha_{i}+\beta_{i}+\gamma_{i}}}
$$

for any $\xi=\left(\xi_{1}, \xi_{2}\right), \eta=\left(\eta_{1}, \eta_{2}\right), \zeta=\left(\zeta_{1}, \zeta_{2}\right) \in \mathbb{R}^{2}$ and sufficiently many multi-indices $\alpha=\left(\alpha_{1}, \alpha_{2}\right), \beta=\left(\beta_{1}, \beta_{2}\right), \gamma=\left(\gamma_{1}, \gamma_{2}\right)$. And define the operator $T_{m}^{0}(f, g, h)\left(x_{1}, x_{2}\right):=\left(\int_{\mathbb{R}^{6}} m(\xi, \eta, \zeta) e^{2 \pi i x(\xi+\eta+\zeta)} \hat{f}(\xi) \hat{g}(\eta) \hat{h}(\zeta) d \xi d \eta d \zeta\right) \varphi_{0}\left(x_{1}\right) \varphi_{0}^{\prime}\left(x_{2}\right)$ 
where $\varphi_{0}, \varphi_{0}^{\prime} \in \mathcal{S}(\mathbb{R})$ are supported on $I^{0}=[-1,1]$. Then for $1<p_{1}, p_{2}, p_{3} \leq \infty$ and $1 / p_{1}+1 / p_{2}+1 / p_{3}=1 / r>0$ there holds

$$
\left\|T_{m}^{0}(f, g, h)\left(x_{1}, x_{2}\right)\right\|_{L^{r}} \lesssim\left\|f \tilde{\chi}_{0} \otimes \tilde{\chi}_{0}\right\|_{p_{1}}\left\|g \tilde{\chi}_{0} \otimes \tilde{\chi}_{0}\right\|_{p_{2}}\left\|h \tilde{\chi}_{0} \otimes \tilde{\chi}_{0}\right\|_{p_{3}},
$$

where $\tilde{\chi}_{0}:=\tilde{\chi}_{I^{0}}$ is defined as (2.1).

We now return to the discussion of the classical single-parameter Coifman-Meyer type operator (1.2) under the condition (1.1). Note that in (1.1) the only singularity for the symbol $m$ is at the origin. In [18, Muscalu considered some types of symbols having flag singularities. More precisely, in the trilinear case, the symbol $m(\xi, \eta, \zeta)$ is a product of two symbols in $\mathcal{M}\left(\mathbb{R}^{3}\right)$ and $\mathcal{M}\left(\mathbb{R}^{2}\right)$ respectively, i.e, $m(\xi, \eta, \zeta)=$ $m_{1}(\xi, \eta, \zeta) m_{2}(\eta, \zeta)$ for $m_{1} \in \mathcal{M}\left(\mathbb{R}^{3}\right), m_{2} \in \mathcal{M}\left(\mathbb{R}^{2}\right)$ satisfying

$$
\begin{aligned}
\left|\partial_{\xi}^{\alpha} \partial_{\eta}^{\beta} \partial_{\zeta}^{\gamma} m_{1}(\xi, \eta, \zeta)\right| & \lesssim \frac{1}{(|\xi|+|\eta|+|\zeta|)^{\alpha+\beta+\gamma}} \\
\left|\partial_{\eta}^{\beta} \partial_{\zeta}^{\gamma} m_{2}(\eta, \zeta)\right| & \lesssim \frac{1}{(|\eta|+|\zeta|)^{\beta+\gamma}}
\end{aligned}
$$

for every $\xi, \eta, \zeta \in \mathbb{R}$ and sufficiently many indices $\alpha, \beta$ and $\gamma$. Define

$$
T_{m_{1}, m_{2}}(f, g, h)(x):=\int_{\mathbb{R}^{3}} m_{1}(\xi, \eta, \zeta) m_{2}(\eta, \zeta) \hat{f}(\xi) \hat{g}(\eta) \hat{h}(\zeta) e^{2 \pi i(\xi+\eta+\zeta) \cdot x} d \xi d \eta d \zeta
$$

where $f, g, h \in \mathcal{S}(\mathbb{R})$. Then there holds

Theorem 1.6. ( [18]) The operator defined in (1.6) maps $L^{p_{1}} \times L^{p_{2}} \times L^{p_{3}} \rightarrow L^{r}$ for $1<p_{1}, p_{2}, p_{3} \leq \infty$ with $1 / p_{1}+1 / p_{2}+1 / p_{3}=1 / r$, and $0<r<\infty$. In particular, the boundedness $L^{\infty} \times L^{\infty} \times L^{p_{3}} \rightarrow L^{p_{3}}, L^{\infty} \times L^{p_{2}} \times L^{\infty} \rightarrow L^{p_{2}}, L^{p_{1}} \times L^{\infty} \times L^{p_{3}} \rightarrow L^{r}$ and $L^{p_{1}} \times L^{p_{2}} \times L^{\infty} \rightarrow L^{r}$ are true.

Moreover, for the above theorem, the estimates like $L^{t} \times L^{\infty} \times L^{\infty} \rightarrow L^{t}$ or $L^{\infty} \times L^{\infty} \times L^{\infty} \rightarrow L^{\infty}$ are false, and these can be checked by setting one of the symbols to be identically 1 . Moreover, the method in [18] can be applied when studying the adjoints of those operators. Also, when $0<p_{1}, p_{2}, p_{3} \leq \infty$, Miyachi and Tomita in [16] proved the boundedness of (1.6) on Hardy and BMO spaces.

The main purpose of this paper is to study the $L^{r}$ estimates for the bi-parameter trilinear Fourier multipliers with flag singularity as defined in (1.6), as well as the corresponding bi-parameter trilinear pseudo-differential variants. We consider the multipliers $m_{1} \in \mathcal{B} \mathcal{M}\left(\mathbb{R}^{6}\right)$ and $m_{2} \in \mathcal{B} \mathcal{M}\left(\mathbb{R}^{4}\right)$ satisfying the following conditions:

$$
\begin{gathered}
\left|\partial_{\xi_{1}}^{\alpha_{1}} \partial_{\xi_{2}}^{\alpha_{2}} \partial_{\eta_{1}}^{\beta_{1}} \partial_{\eta_{2}}^{\beta_{2}} \partial_{\zeta_{1}}^{\gamma_{1}} \partial_{\zeta_{2}}^{\gamma_{2}} m_{1}(\xi, \eta, \zeta)\right| \\
\lesssim \frac{1}{\left(\left|\xi_{1}\right|+\left|\eta_{1}\right|+\left|\zeta_{1}\right|\right)^{\alpha_{1}+\beta_{1}+\gamma_{1}}} \frac{1}{\left(\left|\xi_{2}\right|+\left|\eta_{2}\right|+\left|\zeta_{2}\right|\right)^{\alpha_{2}+\beta_{2}+\gamma_{2}}}, \\
\left|\partial_{\eta_{1}}^{\beta_{1}} \partial_{\eta_{2}}^{\beta_{2}} \partial_{\zeta_{1}}^{\gamma_{1}} \partial_{\zeta_{2}}^{\gamma_{2}} m_{2}(\eta, \zeta)\right| \lesssim \frac{1}{\left(\left|\eta_{1}\right|+\left|\zeta_{1}\right|\right)^{\beta_{1}+\gamma_{1}}} \frac{1}{\left(\left|\eta_{2}\right|+\left|\zeta_{2}\right|\right)^{\beta_{2}+\gamma_{2}}},
\end{gathered}
$$


for every $\xi=\left(\xi_{1}, \xi_{2}\right), \eta=\left(\eta_{1}, \eta_{2}\right), \zeta=\left(\zeta_{1}, \zeta_{2}\right) \in \mathbb{R} \times \mathbb{R}$ and all multi-indices $\alpha=$ $\left(\alpha_{1}, \alpha_{2}\right), \beta=\left(\beta_{1}, \beta_{2}\right)$ and $\gamma=\left(\gamma_{1}, \gamma_{2}\right)$.

Our main theorems are as follows.

Theorem 1.7. For $f, g, h \in \mathcal{S}\left(\mathbb{R}^{2}\right)$, the bi-parameter operators

$$
T_{m_{1}, m_{2}}(f, g, h)(x):=\int_{\mathbb{R}^{6}} m_{1}(\xi, \eta, \zeta) m_{2}(\eta, \zeta) \hat{f}(\xi) \hat{g}(\eta) \hat{h}(\zeta) e^{2 \pi i(\xi+\eta+\zeta) \cdot x} d \xi d \eta d \zeta
$$

would map $L^{p_{1}} \times L^{p_{2}} \times L^{p_{3}} \rightarrow L^{r}$ for $1<p_{1}, p_{2}, p_{3}<\infty$ with $1 / p_{1}+1 / p_{2}+1 / p_{3}=1 / r$ and $0<r<\infty$, if one assumes that the operators

$$
\int_{\mathbb{R}^{6}} m^{\prime}\left(\xi_{1}, \eta_{1}, \zeta_{1}\right) m^{\prime \prime}\left(\eta_{2}, \zeta_{2}\right) \hat{f}(\xi) \hat{g}(\eta) \hat{h}(\zeta) e^{2 \pi i(\xi+\eta+\zeta) \cdot x} d \xi d \eta d \zeta,
$$

satisfy the same Hölder type estimates, where $m^{\prime} \in \mathcal{M}\left(\mathbb{R}^{3}\right)$ and $m^{\prime \prime} \in \mathcal{M}\left(\mathbb{R}^{2}\right)$ are two one-parameter symbols.

In fact, we will reduce (1.7) to a sum of Fourier multipliers, each with different types of symbols; for some of these operators we prove Hölder type estimates, and the remaining operators are treated under an additional assumption about symbols of form (1.8). While the helicoidal method of [1] can be used to treat certain of the operators in our reduction, it does not give the boundedness of (1.8). We conjecture that Hölder-type estimates for these operators are true. More details are in Section 4 and Remark 1 .

The above theorem indicates that providing estimates for the operator (1.7) are the fundamentally new obstacles in obtaining estimates for the operator (1.8). In fact, the proof of this theorem shows that the study of the bi-parameter flag multiplier (1.7) can be essentially reduced to the study of classical bi-parameter multilinear Fourier multipliers like (1.5) as well as the multipliers with tensor product symbols like (1.8).

As it turns out, estimates for the operator (1.7) are of interest even in restricted function spaces. Recently, Muscalu and Zhai ( [23]), and see also [26]) proved the following estimates for (1.7) under a certain tensor product assumption.

Theorem $1.8([23])$. Let $g(x)=g_{1}\left(x_{1}\right) \otimes g_{2}\left(x_{2}\right), h(x)=h_{1}\left(x_{1}\right) \otimes h_{2}\left(x_{2}\right), \frac{1}{p}+\frac{1}{p_{2}}+\frac{1}{p_{3}}=$ $\frac{1}{p}+\frac{1}{q_{2}}+\frac{1}{q_{3}}=\frac{1}{r}$, and $0<r<\infty$. Then (1.7) maps

(a) $L^{p} \times L_{x_{1}}^{p_{2}}\left(L_{x_{2}}^{q_{2}}\right) \times L_{x_{1}}^{p_{3}}\left(L_{x_{2}}^{q_{3}}\right) \rightarrow L^{r}$, for $1<p, p_{2}, p_{3}, q_{2}, q_{3} \leq \infty$ and $\left(p_{2}, p_{3}\right) \neq$ $(\infty, \infty)$ and $\left(q_{2}, q_{3}\right) \neq(\infty, \infty)$.

(b) $L^{p} \times L^{\infty} \times L^{p_{3}} \rightarrow L^{r}$ and $L^{p} \times L^{p_{2}} \times L^{\infty} \rightarrow L^{r}$, for $1<p \leq \infty$ and $1<p_{2}, p_{3}<\infty$.

The methods of [23] used flag paraproducts and some novel and careful stoppingtime arguments. Note that when $1<p, p_{2}, q_{2}, p_{3}, q_{3}<\infty$, our reduction of (1.7) 
essentially into the sum of bi-parameter trilinear multipliers (namely, trilinear version of (1.5)), and our reduced multipliers $m^{\prime}\left(\xi_{1}, \eta_{1}, \zeta_{1}\right) m^{\prime \prime}\left(\eta_{2}, \zeta_{2}\right)$ as in (1.8) can be used to offer an alternative proof for some of these mixed norm estimates as above under the tensor product setting. We include some details in Appendix B. To obtain the mixed norm estimates involving $L^{\infty}$, it seems that further ideas are required and that the arguments of [23] are essential.

In fact, our argument, which gives an alternative proof of the above Theorem 1.8, also allows us to establish the following weighted mixed norm estimates whose proof will be given in Appendix C. We will denote $A_{p}$ as the class of Muckenhoupt weights below and we refer the reader to Appendix $\mathrm{C}$ for definition.

Theorem 1.9. Let $g(x)=g_{1}\left(x_{1}\right) \otimes g_{2}\left(x_{2}\right), h(x)=h_{1}\left(x_{1}\right) \otimes h_{2}\left(x_{2}\right)$. Assume that

$$
w_{1}^{1}\left(x_{1}\right), w_{2}^{1}\left(x_{2}\right) \in A_{p}, w_{1}^{2}\left(x_{1}\right) \in A_{p_{2}}, w_{1}^{3}\left(x_{1}\right) \in A_{p_{3}}, w_{2}^{2}\left(x_{2}\right) \in A_{q_{2}}, w_{2}^{3}\left(x_{2}\right) \in A_{q_{3}},
$$

then (1.7) maps $L^{p}\left(w_{1}^{1} \otimes w_{2}^{1}\right) \times L_{x_{1}}^{p_{2}}\left(w_{1}^{2}\right)\left(L_{x_{2}}^{q_{2}}\left(w_{2}^{2}\right)\right) \times L_{x_{1}}^{p_{3}}\left(w_{1}^{3}\right)\left(L_{x_{2}}^{q_{3}}\left(w_{2}^{3}\right)\right) \rightarrow L^{r}\left(w_{1} \otimes w_{2}\right)$ for $1<p, p_{2}, p_{3}, q_{2}, q_{3}<\infty, 0<r<\infty$ and $\frac{1}{p}+\frac{1}{p_{2}}+\frac{1}{p_{3}}=\frac{1}{p}+\frac{1}{q_{2}}+\frac{1}{q_{3}}=\frac{1}{r}$, where

$$
\begin{aligned}
& w_{1}\left(x_{1}\right)=\left(w_{1}^{1}\right)^{r / p} \cdot\left(w_{1}^{2}\right)^{r / p_{2}} \cdot\left(w_{1}^{3}\right)^{r / p_{3}}, \\
& w_{2}\left(x_{2}\right)=\left(w_{2}^{1}\right)^{r / p} \cdot\left(w_{2}^{2}\right)^{r / q_{2}} \cdot\left(w_{2}^{3}\right)^{r / q_{3}} .
\end{aligned}
$$

In particular, by taking $w_{1}^{1}=w_{1}^{2}=w_{1}^{3}=w_{1} \in A_{\min \left(p, p_{2}, p_{3}\right)}, w_{2}^{1}=w_{2}^{2}=w_{2}^{3}=$ $w_{2} \in A_{\min \left(p, q_{2}, q_{3}\right)}$, (1.7) maps $L^{p}\left(w_{1} \otimes w_{2}\right) \times L_{x_{1}}^{p_{2}}\left(w_{1}\right)\left(L_{x_{2}}^{q_{2}}\left(w_{2}\right)\right) \times L_{x_{1}}^{p_{3}}\left(w_{1}\right)\left(L_{x_{2}}^{q_{3}}\left(w_{2}\right)\right) \rightarrow$ $L^{r}\left(w_{1} \otimes w_{2}\right)$.

Now we state our result for the corresponding bi-parameter trilinear pseudodifferential operators. The one-parameter case was studied in [15]. Let

$$
T_{a b}(f, g, h)(x):=\int_{\mathbb{R}^{6}} a(x, \xi, \eta, \zeta) b(x, \eta, \zeta) \hat{f}(\xi) \hat{g}(\eta) \hat{h}(\zeta) e^{2 \pi i x(\xi+\eta+\zeta)} d \xi d \eta d \zeta
$$

where $f, g, h \in \mathcal{S}\left(\mathbb{R}^{2}\right)$, and the bi-parameter smooth symbols $a, b \in B S_{1,0}^{0}$ satisfy the following conditions

$$
\begin{gathered}
\left|\partial_{x_{1}}^{l_{1}} \partial_{x_{2}}^{l_{2}} \partial_{\xi_{1}}^{\alpha_{1}} \partial_{\xi_{2}}^{\alpha_{2}} \partial_{\eta_{1}}^{\beta_{1}} \partial_{\eta_{2}}^{\beta_{2}} \partial_{\zeta_{1}}^{\gamma_{1}} \partial_{\zeta_{2}}^{\gamma_{2}} a(x, \xi, \eta, \zeta)\right| \\
\lesssim \frac{1}{\left(1+\left|\xi_{1}\right|+\left|\eta_{1}\right|+\left|\zeta_{1}\right|\right)^{\alpha_{1}+\beta_{1}+\gamma_{1}}} \frac{1}{\left(1+\left|\xi_{2}\right|+\left|\eta_{2}\right|+\left|\zeta_{2}\right|\right)^{\alpha_{2}+\beta_{2}+\gamma_{2}}}, \\
\left|\partial_{x_{1}}^{l_{1}} \partial_{x_{2}}^{l_{2}} \partial_{\eta_{1}}^{\beta_{1}} \partial_{\eta_{2}}^{\beta_{2}} \partial_{\zeta_{1}}^{\gamma_{1}} \partial_{\zeta_{2}}^{\gamma_{2}} b(x, \eta, \zeta)\right| \lesssim \frac{1}{\left(1+\left|\eta_{1}\right|+\left|\zeta_{1}\right|\right)^{\beta_{1}+\gamma_{1}}} \frac{1}{\left(1+\left|\eta_{2}\right|+\left|\zeta_{2}\right|\right)^{\beta_{2}+\gamma_{2}}} .
\end{gathered}
$$

for every $x=\left(x_{1}, x_{2}\right), \xi=\left(\xi_{1}, \xi_{2}\right), \eta=\left(\eta_{1}, \eta_{2}\right), \zeta=\left(\zeta_{1}, \zeta_{2}\right) \in \mathbb{R} \times \mathbb{R}$ and all multiindices $\alpha=\left(\alpha_{1}, \alpha_{2}\right), \beta=\left(\beta_{1}, \beta_{2}\right)$ and $\gamma=\left(\gamma_{1}, \gamma_{2}\right)$. We will prove the following estimate when assuming the estimates in Theorem 1.7

Theorem 1.10. The operators $T_{a b}$ defined as (1.9) map $L^{p_{1}} \times L^{p_{2}} \times L^{p_{3}} \rightarrow L^{r}$ for $1<p_{1}, p_{2}, p_{3}<\infty$ with $1 / p_{1}+1 / p_{2}+1 / p_{3}=1 / r$ and $0<r<\infty$, provided that the multiplier operator defined in (1.8) satisfies the same $L^{r}$ estimate. 
The proof of the $L^{r}$ estimates for the bi-parameter trilinear flag Fourier multipliers of Theorem 1.7 proceeds by reducing to a decomposition into multipliers based on the support on the frequency variables, then studying the Hölder type $L^{r}$ estimates for each. Such a reduction is partly inspired by earlier work in both the singleparameter and bi-parameter settings, for instance, [16, 19, 21, 22. To prove Theorem 1.10, we reduce the bi-parameter trilinear pseudo-differential operator to a localized version. Then by taking advantage of the paraproducts studied in [18, 20], but with all dyadic intervals having lengths at most 1, and Theorem 1.7, we prove the Hölder estimates for the localized operator. This is Theorem 5.1 .

The rest of the paper is organized as follows. In Section 2, we collect some notation and definitions used in the paper. Section 4 contains the proof of Theorem 1.7. In Section 5, we show that the main Theorem 1.10 can be reduced to an estimate for a localized operator (Theorem 5.1). In Section 6, we give the proof of Theorem 5.1. In fact, the localized operator will be written as certain bi-parameter paraproducts, where all the involved dyadic intervals have lengths at most 1 . This allows us to avoid the more complicated "size" and "energy" estimates used in [19,21,22] to deal with paraproducts.

Acknowledgement. The authors are grateful to Camil Muscalu for pointing out an error in our first version posted in the arxiv.org and for many useful comments as we were revising the paper. To be precise, the derivation of (4.5) in the original version of this paper was incorrect. Indeed, we do not claim to have a proof of the earlier version of Theorem 1.7. Instead we prove here that the $L^{r}$ estimates for the bi-parameter trilinear flag multiplier can be reduced to the $L^{r}$ estimate for multipliers of the form $m_{1}\left(\xi_{1}, \eta_{1}, \zeta_{1}\right) m_{2}\left(\eta_{2}, \zeta_{2}\right)$. The main revision is in subsection 4.2.2 where we have adapted a new method of reduction of the general bi-parameter trilinear multiplier to the special one of the form $m_{1}\left(\xi_{1}, \eta_{1}, \zeta_{1}\right) m_{2}\left(\eta_{2}, \zeta_{2}\right)$. We also thank Camil Muscalu for communicating the results of Zhai's thesis, which alerted us to the interest in addressing the tensor product case (Theorem 1.8) (See Appendix $\mathrm{B})$.

\section{NOTATIONS AND PRELIMINARIES}

Let $\mathcal{S}\left(\mathbb{R}^{n}\right)$ denote the Schwartz space of rapidly decreasing, $C^{\infty}$ functions in $\mathbb{R}^{n}$. Define the Fourier transform of a function $f$ in $\mathcal{S}\left(\mathbb{R}^{n}\right)$ as

$$
F(f)(\xi)=\hat{f}(\xi)=\int_{\mathbb{R}^{n}} f(x) e^{-2 \pi i x \cdot \xi} d x
$$

extended in the usual way to the space of tempered distribution $\mathcal{S}^{\prime}\left(\mathbb{R}^{n}\right)$, which is the dual space of $\mathcal{S}\left(\mathbb{R}^{n}\right)$. The use $\mathcal{F}^{-1}(f)$ to denote the inverse Fourier transform of $f$. 
Throughout the paper, expressions of the form $A \lesssim B$ are used to mean that there exists a universal constant $C>1$ so that $A \leq C B$, and the notation $A \sim B$ denotes that both $A \lesssim B$ and $B \lesssim A$.

Intervals in the form of $\left[2^{k} n, 2^{k}(n+1)\right]$ in $\mathbb{R}$, where $k, n \in \mathbb{Z}$, are called dyadic intervals; and $\mathbb{D}$ is the set of all such dyadic intervals. Moreover, the occurrence of any of these expressions in this paper means the following: $I^{0}:=[-1,1], I_{n}=J_{n}=$ $I_{n}^{\prime}=J_{n}^{\prime}:=[n, n+1]$ for $n \in \mathbb{Z}$.

Definition 1. For $I \in \mathbb{D}$, we define the approximate cutoff function as

$$
\tilde{\chi}_{I}(x):=\left(1+\frac{\operatorname{dist}(x, I)}{|I|}\right)^{-100}
$$

Definition 2. Let $I \subseteq \mathbb{R}$ be an arbitrary interval. A smooth function $\varphi$ is said to be a bump adapted to $I$ if and only if one has

$$
\left|\varphi^{(l)}\right| \leq C_{l} C_{M} \frac{1}{|I|^{l}} \frac{1}{\left(1+\left|x-x_{I}\right| /|I|\right)^{M}}
$$

for every integer $M \in \mathbb{N}$ and sufficiently many derivatives $l \in \mathbb{N}$, where $x_{I}$ denotes the center of $I$ and $|I|$ is the length of $I$.

If $\varphi_{I}$ is a bump adapted to $I$, we say that $|I|^{-1 / p} \varphi_{I}$ is an $L^{p}$-normalized bump adapted to $I$, for $1 \leq p \leq \infty$.

Definition 3. A sequence of $L^{2}$-normalized bumps $\left(\Phi_{I}\right)_{I \in \mathbb{D}}$ adapted to dyadic intervals $I \in \mathbb{D}$ is called a non-lacunary sequence if and only if for each $I \in \mathbb{D}$ there exists an interval $\omega_{I}=\omega_{|I|}$ symmetric with respect to the origin so that supp $\widehat{\Phi_{I}} \subseteq \omega_{I}$ and $\left|\omega_{I}\right| \sim|I|^{-1}$.

Definition 4. A sequence of $L^{2}$-normalized bumps $\left(\Phi_{I}\right)_{I \in \mathbb{D}}$ adapted to dyadic intervals $I \in \mathbb{D}$ is called a lacunary sequence if and only if for each $I \in \mathbb{D}$ there exists an interval $\omega_{I}=\omega_{|I|}$ so that supp $\widehat{\Phi_{I}} \subseteq \omega_{I},\left|\omega_{I}\right| \sim|I|^{-1} \sim \operatorname{dist}\left(0, \omega_{I}\right)$ and $0 \notin 5 \omega_{I}$.

Definition 5. Let $\mathcal{I}, \mathcal{J} \subseteq \mathbb{D}$ be two families of dyadic intervals that have lengths at most 1. Suppose that $\left(\phi_{I}^{j}\right)_{I \in \mathcal{I}}$ for $j=1,2,3$ are three families of $L^{2}$-normalized bump functions such that the family $\left(\phi_{I}^{2}\right)_{I \in \mathcal{I}}$ is non-lacunary while the families $\left(\phi_{I}^{j}\right)_{I \in \mathcal{I}}$ for $j \neq 2$ are both lacunary, and $\left(\phi_{J}^{j}\right)_{J \in \mathcal{J}}$ for $j=1,2,3$ are three families of $L^{2}$ normalized bump functions, where at least two of the three are lacunary.

We define as in [18] the discrete model operators $T_{1}$ and $T_{1, k_{0}}$ for a positive integer $k_{0}$ by

$$
\begin{gathered}
T_{1}(f, g, h)=\sum_{I \in \mathcal{I}} \frac{1}{|I|^{\frac{1}{2}}}\left\langle f, \phi_{I}^{1}\right\rangle\left\langle B_{I}^{1}(g, h), \phi_{I}^{2}\right\rangle \phi_{I}^{3} \\
\text { where } \quad B_{I}^{1}(g, h)=\sum_{\substack{J \in \mathcal{J} \\
\left|\omega_{J}^{3}\right| \leq\left|\omega_{I}^{2}\right|}} \frac{1}{|J|^{\frac{1}{2}}}\left\langle g, \phi_{J}^{1}\right\rangle\left\langle h, \phi_{J}^{2}\right\rangle \phi_{J}^{3}
\end{gathered}
$$




$$
\begin{gathered}
T_{1, k_{0}}(f, g, h)=\sum_{I \in \mathcal{I}} \frac{1}{|I|^{\frac{1}{2}}}\left\langle f, \phi_{I}^{1}\right\rangle\left\langle B_{I, k_{0}}^{1}(g, h), \phi_{I}^{2}\right\rangle \phi_{I}^{3} \\
\text { where } \quad B_{I, k_{0}}^{1}(g, h)=\sum_{\substack{J \in \mathcal{J} \\
\left|\omega_{J}^{3}\right| \simeq\left|\omega_{I}^{2}\right|}} \frac{1}{|J|^{\frac{1}{2}}}\left\langle g, \phi_{J}^{1}\right\rangle\left\langle h, \phi_{J}^{2}\right\rangle \phi_{J}^{3}
\end{gathered}
$$

\section{A LeibNiz RULe}

Before giving the proof of Theorem 1.7, we give an example where the operator we consider plays a role. This is one of the possible motivations for the study of such operators. The details are included in the Appendix A.

Let $f, g, h \in \mathcal{S}\left(\mathbb{R}^{2}\right)$, and for $\alpha_{1}, \alpha_{2}>0$ define

$$
\widehat{D_{1}^{\alpha_{1}} h}(u)=\left(2 \pi\left|u_{1}\right|\right)^{\alpha_{1}} \hat{h}(u), \quad \widehat{D_{2}^{\alpha_{2}} h}(u)=\left(2 \pi\left|u_{2}\right|\right)^{\alpha_{2}} \hat{h}(u), \quad u=\left(u_{1}, u_{2}\right) \in \mathbb{R}^{2} .
$$

In [20 22], it was proved that the boundedness of the bi-parameter bilinear Fourier multiplier in Theorem 1.2 implies the following Leibniz rule.

$$
\begin{aligned}
\left\|D_{1}^{\alpha_{1}} D_{2}^{\alpha_{2}}(f g)\right\|_{L^{r}} \lesssim & \left\|D_{1}^{\alpha_{1}} D_{2}^{\alpha_{2}} f\right\|_{L^{p_{1}}}\|g\|_{L^{q_{1}}}+\left\|D_{1}^{\alpha_{1}} f\right\|_{L^{p_{2}}}\left\|D_{2}^{\alpha_{2}} g\right\|_{L^{q_{2}}} \\
& +\left\|D_{2}^{\alpha_{2}} f\right\|_{L^{p_{3}}}\left\|D_{1}^{\alpha_{1}} g\right\|_{L^{q_{3}}}+\|f\|_{L^{p_{4}}}\left\|D_{1}^{\alpha_{1}} D_{2}^{\alpha_{2}} g\right\|_{L^{q_{4}}},
\end{aligned}
$$

where $1 / p_{i}+1 / q_{i}=1 / r, 1<p_{i}, q_{i} \leq \infty$ for $i=1,2,3,4$ and $\max \left(\frac{1}{1+\alpha_{1}}, \frac{1}{1+\alpha_{2}}\right)<r<$ $\infty$ (one can refer to [20 22] to see how such restrictions appear).

Then it's very natural and interesting to ask if such bi-parameter Leibniz rule holds when there is higher complexity of the differentiation. In particular, a Leibniz estimate for an expression like the following relies on our theorem:

$$
\left\|D_{1}^{\alpha_{1}} D_{2}^{\alpha_{2}}\left(f \cdot D_{1}^{\beta_{1}} D_{2}^{\beta_{2}}(g h)\right)\right\|_{L^{r}},
$$

where $\max \left(\frac{1}{1+\alpha_{1}}, \frac{1}{1+\alpha_{2}}, \frac{1}{1+\beta_{1}}, \frac{1}{1+\beta_{2}}\right)<r<\infty$. First note that an iteration of (3.1) results in the inequality

$$
\begin{aligned}
& \left\|D_{1}^{\alpha_{1}} D_{2}^{\alpha_{2}}\left(f \cdot D_{1}^{\beta_{1}} D_{2}^{\beta_{2}}(g h)\right)\right\|_{L^{r}} \\
\lesssim & \left\|D_{1}^{\alpha_{1}} D_{2}^{\alpha_{2}} f\right\|_{L^{p_{1}}}\left\|D_{1}^{\beta_{1}} D_{2}^{\beta_{2}}(g h)\right\|_{L^{t_{1}}}+\left\|D_{1}^{\alpha_{1}} f\right\|_{L^{p_{2}}}\left\|D_{1}^{\beta_{1}} D_{2}^{\alpha_{2}+\beta_{2}}(g h)\right\|_{L^{t_{2}}} \\
& +\left\|D_{2}^{\alpha_{2}} f\right\|_{L^{p_{3}}}\left\|D_{1}^{\alpha_{1}+\beta_{1}} D_{2}^{\beta_{2}}(g h)\right\|_{L^{t_{3}}}+\|f\|_{L^{p_{4}}}\left\|D_{1}^{\alpha_{1}+\beta_{1}} D_{2}^{\alpha_{2}+\beta_{2}}(g h)\right\|_{L^{t_{4}}},
\end{aligned}
$$

where $1 / r=1 / p_{i}+1 / t_{i}$ for $i=1,2,3,4$. However, this argument using (3.1) requires $t_{i}>1$, while the ideal restriction is $t_{i}>\frac{1}{2}$, since we expect to further expand the differentiation on $g h$. Thus, it's a non-trivial question to get a general Leibniz rule for (3.2), that is

$$
\begin{aligned}
& \left\|D_{1}^{\alpha_{1}} D_{2}^{\alpha_{2}}\left(f \cdot D_{1}^{\beta_{1}} D_{2}^{\beta_{2}}(g h)\right)\right\|_{L^{r}} \\
\lesssim & \left\|D_{1}^{\alpha_{1}} D_{2}^{\alpha_{2}} f\right\|_{L^{p_{1}}} \cdot\left\|D_{1}^{\beta_{1}} D_{2}^{\beta_{2}} g\right\|_{L^{q_{1}}} \cdot\|h\|_{L^{s_{1}}}+\left\|D_{1}^{\alpha_{1}} D_{2}^{\alpha_{2}} f\right\|_{L^{p_{2}}} \cdot\left\|D_{1}^{\beta_{1}} g\right\|_{L^{q_{2}}} \cdot\left\|D_{2}^{\beta_{2}} h\right\|_{L^{s_{2}}} \\
& +\left\|D_{1}^{\alpha_{1}} D_{2}^{\alpha_{2}} f\right\|_{L^{p_{3}}} \cdot\left\|D_{2}^{\beta_{2}} g\right\|_{L^{q_{3}}} \cdot\left\|D_{1}^{\beta_{1}} h\right\|_{L^{s_{3}}}+\left\|D_{1}^{\alpha_{1}} D_{2}^{\alpha_{2}} f\right\|_{L^{p_{4}}} \cdot\|g\|_{L^{q_{4}}} \cdot\left\|D_{1}^{\beta_{1}} D_{2}^{\beta_{2}} h\right\|_{L^{s_{4}}} \\
& +\left\|D_{1}^{\alpha_{1}} f\right\|_{L^{p_{5}}} \cdot\left\|D_{1}^{\beta_{1}} D_{2}^{\alpha_{2}+\beta_{2}} g\right\|_{L^{q_{5}}} \cdot\|h\|_{L^{s_{5}}}+\left\|D_{1}^{\alpha_{1}} f\right\|_{L^{p_{6}}} \cdot\left\|D_{1}^{\beta_{1}} g\right\|_{L^{q_{6}}} \cdot\left\|D_{2}^{\alpha_{2}+\beta_{2}} h\right\|_{L^{s_{6}}}
\end{aligned}
$$




$$
\begin{aligned}
& +\left\|D_{1}^{\alpha_{1}} f\right\|_{L^{p_{7}}} \cdot\left\|D_{2}^{\alpha_{2}+\beta_{2}} g\right\|_{L^{q_{7}}} \cdot\left\|D_{1}^{\beta_{1}} h\right\|_{L^{s_{7}}}+\left\|D_{1}^{\alpha_{1}} f\right\|_{L^{p_{8}}} \cdot\|g\|_{L^{q_{8}}} \cdot\left\|D_{1}^{\beta_{1}} D_{2}^{\alpha_{2}+\beta_{2}} h\right\|_{L^{s_{8}}} \\
& +\left\|D_{2}^{\alpha_{2}} f\right\|_{L^{p_{9}}} \cdot\left\|D_{1}^{\alpha_{1}+\beta_{1}} D_{2}^{\beta_{2}} g\right\|_{L^{q_{9}}} \cdot\|h\|_{L^{s_{9}}}+\left\|D_{2}^{\alpha_{2}} f\right\|_{L^{p_{10}}} \cdot\left\|D_{1}^{\alpha_{1}+\beta_{1}} g\right\|_{L^{q_{10}}} \cdot\left\|D_{2}^{\beta_{2}} h\right\|_{L^{s_{10}}} \\
& +\left\|D_{2}^{\alpha_{2}} f\right\|_{L^{p_{11}}} \cdot\left\|D_{2}^{\beta_{2}} g\right\|_{L^{q_{11}}} \cdot\left\|D_{1}^{\alpha_{1}+\beta_{1}} h\right\|_{L^{s_{11}}}+\left\|D_{2}^{\alpha_{2}} f\right\|_{L^{p_{12}}} \cdot\|g\|_{L^{q_{12}}} \cdot\left\|D_{1}^{\alpha_{1}+\beta_{1}} D_{2}^{\beta_{2}} h\right\|_{L^{s_{12}}} \\
& +\|f\|_{L^{p_{13}}} \cdot\left\|D_{1}^{\alpha_{1}+\beta_{1}} D_{2}^{\alpha_{2}+\beta_{2}} g\right\|_{L^{q_{13}}} \cdot\|h\|_{L^{s_{13}}}+\|f\|_{L^{p_{14}}} \cdot\left\|D_{1}^{\alpha_{1}+\beta_{1}} g\right\|_{L^{q_{14}}} \cdot\left\|D_{2}^{\alpha_{2}+\beta_{2}} h\right\|_{L^{s_{14}}} \\
& +\|f\|_{L^{p_{15}}} \cdot\left\|D_{2}^{\alpha_{2}+\beta_{2}} g\right\|_{L^{q_{15}}} \cdot\left\|D_{1}^{\alpha_{1}+\beta_{1}} h\right\|_{L^{s_{15}}}+\|f\|_{L^{p_{16}}} \cdot\|g\|_{L^{q_{16}}}\left\|D_{1}^{\alpha_{1}+\beta_{1}} D_{2}^{\alpha_{2}+\beta_{2}} h\right\|_{L^{s_{16}}},
\end{aligned}
$$

where $1 / p_{i}+1 / q_{i}+1 / s_{i}=1 / r, 1<p_{i}, q_{i}, s_{i}<\infty$ for $i=1, \ldots, 16$ and $\max \left(\frac{1}{1+\alpha_{1}}, \frac{1}{1+\alpha_{2}}, \frac{1}{1+\beta_{1}}, \frac{1}{1+\beta_{2}}\right)<$ $r<\infty$.

It turns out, we can write $D_{1}^{\alpha_{1}} D_{2}^{\alpha_{2}}\left(f \cdot D_{1}^{\beta_{1}} D_{2}^{\beta_{2}}(g h)\right)$ as a sum of essentially two types of Fourier multipliers. More precisely, we can write

$$
D_{1}^{\alpha_{1}} D_{2}^{\alpha_{2}}\left(f \cdot D_{1}^{\beta_{1}} D_{2}^{\beta_{2}}(g h)\right)=T_{m_{1}, m_{2}}(f, g, h)+T_{m_{3}, m_{4}}(f, g, h)
$$

where $T_{m_{1}, m_{2}}$ is the operator (1.7) in Theorem 1.7, and

$$
T_{m_{3}, m_{4}}(f, g, h)=\int_{\mathbb{R}^{6}} m_{3}(\xi, \eta) m_{4}(\eta, \zeta) \hat{f}(\xi) \hat{g}(\eta) \hat{h}(\zeta) d \xi d \eta d \zeta,
$$

with $m_{3}, m_{4} \in \mathcal{B M}\left(\mathbb{R}^{4}\right)$. Thus, in order to get the Leibniz estimate, we just need to show the Hölder $L^{r}$ estimate for each of the above two operators implies those pieces in (3.3).

Now let's take a quick look at how the estimate in Theorem 1.7 is associated with the 16 terms appearing in (3.3). We indicate some key steps here, and more details can be found in Appendix $\mathrm{A}$. Let $\psi \in \mathcal{S}(\mathbb{R})$ be a Schwartz function satisfying $\operatorname{supp} \hat{\psi} \subseteq\{1 / 2 \leq|u| \leq 2\}$ and

$$
1=\sum_{k \in \mathbb{Z}} \widehat{\psi}_{k}(u), \quad u \neq 0
$$

Now $f \cdot g \cdot h$ can be rewritten by using

$$
\begin{gathered}
\hat{f}(\xi) \hat{g}(\eta) \hat{h}(\zeta)= \\
\left(\sum_{j_{1}, j_{2}} \widehat{\psi}_{j_{1}}\left(\xi_{1}\right) \widehat{\psi}_{j_{2}}\left(\xi_{2}\right) \hat{f}(\xi)\right)\left(\sum_{k_{1}, k_{2}} \widehat{\psi}_{k_{1}}\left(\eta_{1}\right) \widehat{\psi}_{k_{2}}\left(\eta_{2}\right) \hat{g}(\eta)\right)\left(\sum_{l_{1}, l_{2}} \widehat{\psi}_{l_{1}}\left(\zeta_{1}\right) \widehat{\psi}_{l_{2}}\left(\zeta_{2}\right) \hat{h}(\zeta)\right) .
\end{gathered}
$$

Then by using a sequence of appropriate reductions, it turns out that our goal $D_{1}^{\alpha_{1}} D_{2}^{\alpha_{2}}\left(f \cdot D_{1}^{\beta_{1}} D_{2}^{\beta_{2}}(g h)\right)$ can be written as a summation of terms that includes, for example,

$$
D_{1}^{\alpha_{1}} D_{2}^{\alpha_{2}}\left(\sum_{j_{1}, j_{2}}\left(\left(f *\left(\psi_{j_{1}} \otimes \psi_{j_{2}}\right)\right)\left(D_{1}^{\beta_{1}} D_{2}^{\beta_{2}} \Pi_{1}(g, h) *\left(\varphi_{j_{1}} \otimes \varphi_{j_{2}}\right)\right)\right) *\left(\psi_{j_{1}} \otimes \psi_{j_{2}}\right)\right)
$$




$$
=\sum_{j_{1}, j_{2}}\left(\left(D_{1}^{\alpha_{1}} D_{2}^{\alpha_{2}} f *\left(\psi_{j_{1}}^{\prime \prime} \otimes \psi_{j_{2}}^{\prime \prime}\right)\right)\left(D_{1}^{\beta_{1}} D_{2}^{\beta_{2}} \Pi_{1}(g, h) *\left(\varphi_{j_{1}} \otimes \varphi_{j_{2}}\right)\right)\right) *\left(\psi_{j_{1}}^{\prime} \otimes \psi_{j_{2}}^{\prime}\right) .
$$

where $D_{1}^{\beta_{1}} D_{2}^{\beta_{2}} \Pi_{1}(g, h)$ has the form

$$
\sum_{k_{1} \ll j_{1}} \sum_{k_{2} \ll j_{2}}\left(\left(D_{1}^{\beta_{1}} D_{2}^{\beta_{2}} g *\left(\psi_{k_{1}}^{\prime \prime} \otimes \psi_{k_{2}}^{\prime \prime}\right)\right) \cdot\left(h *\left(\varphi_{k_{1}} \otimes \varphi_{k_{2}}\right)\right)\right) *\left(\psi_{k_{1}}^{\prime} \otimes \psi_{k_{2}}^{\prime}\right) .
$$

Here for $i=1,2, k_{i} \ll j_{i}$ means $k_{i}<j_{i}-100, \varphi_{k_{i}}=\sum_{l_{i} \ll k_{i}} \psi_{l_{i}}$, and $\psi_{k_{i}}$ may actually represent $\sum_{k_{i}-100 \leq l_{i} \leq k_{i}+100} \psi_{l_{i}}$. Moreover, $\widehat{\psi}_{k_{i}}^{\prime}(u)=\widehat{\psi}_{k_{i}}(u)\left|\frac{u}{2^{k_{i}}}\right|^{\beta_{i}}, \widehat{\psi}_{k_{i}}^{\prime \prime}(u)=$ $\widehat{\psi}_{k_{i}}(u)\left|\frac{2^{k_{i}}}{u}\right|^{\beta_{i}}$, and $\otimes$ represents the tensor product.

In fact, the expression (3.5) is a Fourier multiplier with symbol having the form $m_{1}(\xi, \eta, \zeta) m_{2}(\eta, \zeta)$, where $m_{1} \in \mathcal{B} \mathcal{M}\left(\mathbb{R}^{6}\right)$ and $m_{2} \in \mathcal{B} \mathcal{M}\left(\mathbb{R}^{4}\right)$ respectively. Then one can see the boundedness of (1.7) in Theorem (1.7) implies the bound

$$
\left\|D_{1}^{\alpha_{1}} D_{2}^{\alpha_{2}} f\right\|_{L^{p_{1}}} \cdot\left\|D_{1}^{\beta_{1}} D_{2}^{\beta_{2}} g\right\|_{L^{q_{1}}} \cdot\|h\|_{L^{s_{1}}},
$$

which appears on the right hand side of (3.3). To see how to get the other terms in (3.3) from the boundedness of (1.7), we need to look at the terms that are similar to (3.5), which appear in the process of reduction. Here we just give one more example,

$$
\begin{aligned}
& D_{1}^{\alpha_{1}} D_{2}^{\alpha_{2}}\left(\sum_{j_{1}, j_{2}}\left(\left(f *\left(\psi_{j_{1}} \otimes \varphi_{j_{2}}\right)\right)\left(D_{1}^{\beta_{1}} D_{2}^{\beta_{2}} \Pi_{2}(g, h) *\left(\varphi_{j_{1}} \otimes \psi_{j_{2}}\right)\right)\right) *\left(\psi_{j_{1}} \otimes \psi_{j_{2}}\right)\right) \\
= & \sum_{j_{1}, j_{2}}\left(\left(D_{1}^{\alpha_{1}} f *\left(\psi_{j_{1}}^{\prime \prime} \otimes \varphi_{j_{2}}\right)\right)\left(D_{1}^{\beta_{1}} D_{2}^{\alpha_{2}+\beta_{2}} \Pi_{2}(g, h) *\left(\varphi_{j_{1}} \otimes \psi_{j_{2}}^{\prime \prime}\right)\right)\right) *\left(\psi_{j_{1}}^{\prime} \otimes \psi_{j_{2}}^{\prime}\right) .
\end{aligned}
$$

where $D_{1}^{\beta_{1}} D_{2}^{\alpha_{2}+\beta_{2}} \Pi_{2}(g, h)$ has the form

$$
\sum_{k_{1} \ll j_{1}} \sum_{k_{2} \ll j_{2}}\left(\left(D_{2}^{\beta_{2}+\alpha_{2}} g *\left(\varphi_{k_{1}} \otimes \psi_{k_{2}}^{\prime \prime}\right)\right) \cdot\left(D_{1}^{\beta_{1}} h *\left(\varphi_{k_{1}} \otimes \psi_{k_{2}}^{\prime \prime}\right)\right)\right) *\left(\psi_{k_{1}}^{\prime} \otimes \psi_{k_{2}}^{\prime}\right) .
$$

As before, (3.6) corresponds to an operator with the symbol $m_{1}(\xi, \eta, \zeta) m_{2}(\eta, \zeta)$, and its boundedness gives

$$
\left\|D_{1}^{\alpha_{1}} f\right\|_{L^{p_{7}}} \cdot\left\|D_{2}^{\alpha_{2}+\beta_{2}} g\right\|_{L^{q_{7}}} \cdot\left\|D_{1}^{\beta_{1}} h\right\|_{L^{s_{7}}} .
$$

However, there are still terms in the reduction that can not be covered by the operators (1.7). Such terms appear when, for example, $j_{1} \gg k_{1} \gg l_{1}, j_{2} \ll k_{2} \ll l_{2}$ in (3.4), and they actually correspond to the operator $T_{m_{3}, m_{4}}$. Thus, in order to obtain the final goal (3.3), one also needs the Hölder type $L^{r}$ estimate for $T_{m_{3}, m_{4}}$. Note that the bi-parameter symbol $m_{3}(\xi, \eta) m_{4}(\eta, \zeta)$ is more singular than the one in $T_{m_{1}, m_{2}}$, and thus it is a more challenging task to obtain Hölder type estimates for the 
associated operator. Overcoming this obstacle is another issue for future research in this subject.

\section{Proof of Theorem 1.7}

4.1. Reduction of the symbols. To prove this theorem, we start with the decomposition and reduction of the symbols

$$
m(\xi, \eta, \zeta):=m_{1}(\xi, \eta, \zeta) m_{2}(\eta, \zeta) .
$$

We take smooth homogeneous functions $\phi_{0}$ and $\phi_{1}$ on $\mathbb{R}^{3} \backslash\{0\}$ such that $\phi_{0}(u, v, w)+$ $\phi_{1}(u, v, w)=1$ and

$$
\operatorname{supp} \phi_{0} \subset\{(u, v, w)|| v|+| w|\leq \epsilon| u \mid\}, \quad \operatorname{supp} \phi_{1} \subset\left\{(u, v, w)|| v|+| w\left|\geq \frac{1}{2} \epsilon\right| u \mid\right\}
$$

for some small $\epsilon$. Then we decompose $m_{1}$ as

$$
\begin{aligned}
& m_{1}=m_{1}\left(\phi_{0}\left(\xi_{1}, \eta_{1}, \zeta_{1}\right)+\phi_{1}\left(\xi_{1}, \eta_{1}, \zeta_{1}\right)\right)\left(\phi_{0}\left(\xi_{2}, \eta_{2}, \zeta_{2}\right)+\phi_{1}\left(\xi_{2}, \eta_{2}, \zeta_{2}\right)\right) \\
= & m_{1} \phi_{0}\left(\xi_{1}, \eta_{1}, \zeta_{1}\right) \phi_{0}\left(\xi_{2}, \eta_{2}, \zeta_{2}\right)+m_{1} \phi_{0}\left(\xi_{1}, \eta_{1}, \zeta_{1}\right) \phi_{1}\left(\xi_{2}, \eta_{2}, \zeta_{2}\right) \\
& +m_{1} \phi_{1}\left(\xi_{1}, \eta_{1}, \zeta_{1}\right) \phi_{0}\left(\xi_{2}, \eta_{2}, \zeta_{2}\right)+m_{1} \phi_{1}\left(\xi_{1}, \eta_{1}, \zeta_{1}\right) \phi_{1}\left(\xi_{2}, \eta_{2}, \zeta_{2}\right) \\
:= & m_{0,0}+m_{0,1}+m_{1,0}+m_{1,1}
\end{aligned}
$$

Obviously $m_{1,1}(\xi, \eta, \zeta) m_{2}(\eta, \zeta)$ satisfies the condition

$$
\begin{aligned}
& \left|\partial_{\xi_{1}}^{\alpha_{1}} \partial_{\xi_{2}}^{\alpha_{2}} \partial_{\eta_{1}}^{\beta_{1}} \partial_{\eta_{2}}^{\beta_{2}} \partial_{\zeta_{1}}^{\gamma_{1}} \partial_{\zeta_{2}}^{\gamma_{2}}\left(m_{1,1}(\xi, \eta, \zeta) m_{2}(\eta, \zeta)\right)\right| \\
& \lesssim \frac{1}{\left(\left|\xi_{1}\right|+\left|\eta_{1}\right|+\left|\zeta_{1}\right|\right)^{\alpha_{1}+\beta_{1}+\gamma_{1}}} \frac{1}{\left(\left|\xi_{2}\right|+\left|\eta_{2}\right|+\left|\zeta_{2}\right|\right)^{\alpha_{2}+\beta_{2}+\gamma_{2}}}
\end{aligned}
$$

and the desired estimate follows from the multilinear version of Theorem 1.2 .

$m_{0,1}$ and $m_{1,0}$ satisfy the similar conditions. For example,

$$
\lesssim \sum_{\substack{\beta_{1}^{\prime}+\beta_{1}^{\prime \prime}=\beta_{1} \\ \gamma_{1}^{\prime}+\gamma_{1}^{\prime \prime}=\gamma_{1}}} \frac{\left|\partial_{\xi_{1}}^{\alpha_{1}} \partial_{\xi_{2}}^{\alpha_{2}} \partial_{\eta_{1}}^{\beta_{1}} \partial_{\eta_{2}}^{\beta_{2}} \partial_{\zeta_{1}}^{\gamma_{1}} \partial_{\zeta_{2}}^{\gamma_{2}}\left(m_{0,1}(\xi, \eta, \zeta) m_{2}(\eta, \zeta)\right)\right|}{\left(\left|\xi_{1}\right|+\left|\eta_{1}\right|+\left|\zeta_{1}\right|\right)^{\alpha_{1}+\beta_{1}^{\prime}+\gamma_{1}^{\prime}}} \frac{1}{\left(\left|\eta_{1}\right|+\left|\zeta_{1}\right|\right)^{\beta_{1}^{\prime \prime}+\gamma_{1}^{\prime \prime}}} \frac{1}{\left(\left|\xi_{2}\right|+\left|\eta_{2}\right|+\left|\zeta_{2}\right|\right)^{\alpha_{2}+\beta_{2}+\gamma_{2}}},
$$

Note that such a condition is stronger than what $m_{0,0}(\xi, \eta, \zeta) m_{2}(\eta, \zeta)$ satisfies, since the estimate for the second parameter is in a classical type. More precisely,

$$
\begin{aligned}
& \left|\partial_{\xi_{1}}^{\alpha_{1}} \partial_{\xi_{2}}^{\alpha_{2}} \partial_{\eta_{1}}^{\beta_{1}} \partial_{\eta_{2}}^{\beta_{2}} \partial_{\zeta_{1}}^{\gamma_{1}} \partial_{\zeta_{2}}^{\gamma_{2}}\left(m_{0,0}(\xi, \eta, \zeta) m_{2}(\eta, \zeta)\right)\right| \\
& \lesssim \sum_{\substack{\beta_{1}^{\prime}+\beta_{1}^{\prime \prime}=\beta_{1} \\
\gamma_{1}^{\prime}+\gamma_{1}^{\prime \prime}=\gamma_{1}}} \sum_{\beta_{2}^{\prime}+\beta_{2}^{\prime \prime}=\gamma_{2}^{\prime \prime}=\gamma_{2}} \frac{1}{\left(\left|\xi_{1}\right|+\left|\eta_{1}\right|+\left|\zeta_{1}\right|\right)^{\alpha_{1}+\beta_{1}^{\prime}+\gamma_{1}^{\prime}}} \frac{1}{\left(\left|\eta_{1}\right|+\left|\zeta_{1}\right|\right)^{\beta_{1}^{\prime \prime}+\gamma_{1}^{\prime \prime}}}
\end{aligned}
$$




$$
\cdot \frac{1}{\left(\left|\xi_{2}\right|+\left|\eta_{2}\right|+\left|\zeta_{2}\right|\right)^{\alpha_{2}+\beta_{2}^{\prime}+\gamma_{2}^{\prime}}} \frac{1}{\left(\left|\eta_{2}\right|+\left|\zeta_{2}\right|\right)^{\beta_{2}^{\prime \prime}+\gamma_{2}^{\prime \prime}}} .
$$

Thus, if suffices to study $m_{0,0}(\xi, \eta, \zeta) m_{2}(\eta, \zeta)$.

We choose $\widehat{\psi}(u) \in \mathcal{S}(\mathbb{R})$ such that $\operatorname{supp} \widehat{\psi} \subseteq\{u \in \mathbb{R}: 1 / 2 \leq|u| \leq 2\}$, and

$$
\sum_{j \in \mathbb{Z}} \widehat{\psi}_{j}(u):=\sum_{j \in \mathbb{Z}} \widehat{\psi}\left(\frac{u}{2^{j}}\right)=1 \quad \text { for } u \neq 0 .
$$

We define $\varphi$ by

$$
\widehat{\varphi}(u)=\sum_{k=-\infty}^{-3} \widehat{\psi}\left(2^{-k} u\right) \text { for } u \neq 0, \quad \widehat{\varphi}(0)=1
$$

which implies

$$
\operatorname{supp} \widehat{\varphi} \subset\left\{u \in \mathbb{R}:|u| \leq 2^{-2}\right\}, \quad \text { and } \widehat{\varphi}(u)=1 \text { for }|u| \leq 2^{-3} .
$$

We also set

$$
\chi(u, v, w)=\sum_{j \in \mathbb{Z}} \widehat{\psi}\left(2^{-j} u\right) \widehat{\varphi}\left(2^{-j+10} v\right) \widehat{\varphi}\left(2^{-j+10} w\right) .
$$

Note that $\chi \in \mathcal{M}\left(\mathbb{R}^{3}\right)$ and $\chi\left(\xi_{1}, \eta_{1}, \zeta_{1}\right)=1, \chi\left(\xi_{2}, \eta_{2}, \zeta_{2}\right)=1$ on $\operatorname{supp} m_{0,0}$.

Since it is sufficient to consider $m_{0,0}(\xi, \eta, \zeta) m_{2}(\eta, \zeta)$, we now use $m_{1}$ to represent $m_{0,0}$. From Taylor's theorem, we have:

$$
\begin{aligned}
m_{1}(\xi, \eta, \zeta)= & \sum_{\beta_{2}^{\prime}+\gamma_{2}^{\prime}<N} \frac{\eta_{2}^{\beta_{2}^{\prime}} \zeta_{2}^{\gamma_{2}^{\prime}}}{\beta_{2}^{\prime} ! \gamma_{2}^{\prime} !} \partial_{\eta_{2}}^{\beta_{2}^{\prime}} \partial_{\zeta_{2}}^{\gamma_{2}^{\prime}} m_{1}\left(\xi, \eta_{1}, 0, \zeta_{1}, 0\right) \\
& +N \sum_{\beta_{2}^{\prime}+\gamma_{2}^{\prime}=N} \frac{\eta_{2}^{\beta_{2}^{\prime}} \zeta_{2}^{\gamma_{2}^{\prime}}}{\beta_{2}^{\prime} ! \gamma_{2}^{\prime} !} \int_{0}^{1}(1-t)^{N-1} \partial_{\eta_{2}}^{\beta_{2}^{\prime}} \partial_{\zeta_{2}}^{\gamma_{2}^{\prime}} m_{1}\left(\xi, \eta_{1}, t \eta_{2}, \zeta_{1}, t \zeta_{2}\right) d t
\end{aligned}
$$

and

$$
\begin{aligned}
m_{1}(\xi, \eta, \zeta)= & \sum_{\beta_{1}^{\prime}+\gamma_{1}^{\prime}<N} \frac{\eta_{1}^{\beta_{1}^{\prime}} \zeta_{1}^{\gamma_{1}^{\prime}}}{\beta_{1}^{\prime} ! \gamma_{1}^{\prime} !} \partial_{\eta_{1}}^{\beta_{1}^{\prime}} \partial_{\zeta_{1}}^{\gamma_{1}^{\prime}} m_{1}\left(\xi, 0, \eta_{2}, 0, \zeta_{2}\right) \\
& +N \sum_{\beta_{1}^{\prime}+\gamma_{1}^{\prime}=N} \frac{\eta_{1}^{\beta_{1}^{\prime}} \zeta_{1}^{\gamma_{1}^{\prime}}}{\beta_{1}^{\prime} ! \gamma_{1}^{\prime} !} \int_{0}^{1}(1-s)^{N-1} \partial_{\eta_{1}}^{\beta_{1}^{\prime}} \partial_{\zeta_{1}}^{\gamma_{1}^{\prime}} m_{1}\left(\xi, s \eta_{1}, \eta_{2}, s \zeta_{1}, \zeta_{2}\right) d s .
\end{aligned}
$$

This gives the expression

$$
=\sum_{\beta_{1}^{\prime}+\gamma_{1}^{\prime}<N} \sum_{\beta_{2}^{\prime}+\gamma_{2}^{\prime}<N} \frac{\eta_{1}^{\beta_{1}^{\prime}} \zeta_{1}^{\gamma_{1}^{\prime}}}{\beta_{1}^{\prime} ! \gamma_{1}^{\prime} !} \frac{\eta_{2}^{\beta_{2}^{\prime}} \zeta_{2}^{\gamma_{2}^{\prime}}}{\beta_{2}^{\prime} ! \gamma_{2}^{\prime} !} \partial_{\eta_{1}}^{\beta_{1}^{\prime}} \partial_{\eta_{2}}^{\beta_{2}^{\prime}} \partial_{\zeta_{1}}^{\gamma_{1}^{\prime}} \partial_{\zeta_{2}}^{\gamma_{2}^{\prime}} m_{1}(\xi, 0,0)
$$




$$
\begin{aligned}
& +N \sum_{\beta_{1}^{\prime}+\gamma_{1}^{\prime}<N} \sum_{\beta_{2}^{\prime}+\gamma_{2}^{\prime}=N} \frac{\eta_{1}^{\beta_{1}^{\prime}} \zeta_{1}^{\gamma_{1}^{\prime}}}{\beta_{1}^{\prime} ! \gamma_{1}^{\prime} !} \frac{\eta_{2}^{\beta_{2}^{\prime}} \zeta_{2}^{\gamma_{2}^{\prime}}}{\beta_{2}^{\prime} ! \gamma_{2}^{\prime} !} \int_{0}^{1}(1-t)^{N-1} \partial_{\eta_{1}}^{\beta_{1}^{\prime}} \partial_{\eta_{2}}^{\beta_{2}^{\prime}} \partial_{\zeta_{1}}^{\gamma_{1}^{\prime}} \partial_{\zeta_{2}}^{\gamma_{2}^{\prime}} m_{1}\left(\xi, 0, t \eta_{2}, 0, t \zeta_{2}\right) d t \\
& +N \sum_{\beta_{1}^{\prime}+\gamma_{1}^{\prime}=N} \sum_{\beta_{2}^{\prime}+\gamma_{2}^{\prime}<N} \frac{\eta_{1}^{\beta_{1}^{\prime}} \zeta_{1}^{\gamma_{1}^{\prime}}}{\beta_{1}^{\prime} ! \gamma_{1}^{\prime} !} \frac{\eta_{2}^{\beta_{2}^{\prime}} \zeta_{2}^{\gamma_{2}^{\prime}}}{\beta_{2}^{\prime} ! \gamma_{2}^{\prime} !} \int_{0}^{1}(1-s)^{N-1} \partial_{\eta_{1}}^{\beta_{1}^{\prime}} \partial_{\eta_{2}}^{\beta_{2}^{\prime}} \partial_{\zeta_{1}}^{\gamma_{1}^{\prime}} \partial_{\zeta_{2}}^{\gamma_{2}^{\prime}} m_{1}\left(\xi, s \eta_{1}, 0, s \zeta_{1}, 0\right) d t \\
& +N^{2} \sum_{\beta_{1}^{\prime}+\gamma_{1}^{\prime}=N} \sum_{\beta_{2}^{\prime}+\gamma_{2}^{\prime}=N} \frac{\eta_{1}^{\beta_{1}^{\prime}} \zeta_{1}^{\gamma_{1}^{\prime}}}{\beta_{1}^{\prime} ! \gamma_{1}^{\prime} !} \frac{\eta_{2}^{\beta_{2}^{\prime}} \zeta_{2}^{\gamma_{2}^{\prime}}}{\beta_{2}^{\prime} ! \gamma_{2}^{\prime} !} \int_{0}^{1} \int_{0}^{1}(1-s)^{N-1}(1-t)^{N-1} \\
& :=m_{1}^{1,1}(\xi, \eta, \zeta)+m_{1}^{1,2}(\xi, \eta, \zeta)+m_{1}^{2,1}(\xi, \eta, \zeta)+m_{1}^{2,2}(\xi, \eta, \zeta)
\end{aligned}
$$

These computations imply that our original symbol $m_{1}(\xi, \eta, \zeta) m_{2}(\eta, \zeta)$ can actually be reduced to

$$
\left(m_{1}^{1,1}(\xi, \eta, \zeta)+m_{1}^{1,2}(\xi, \eta, \zeta)+m_{1}^{2,1}(\xi, \eta, \zeta)+m_{1}^{2,2}(\xi, \eta, \zeta)\right) m_{2}(\eta, \zeta) .
$$

In the following subsections, we deal with each of these four types of symbols.

\subsubsection{The symbol $m_{1}^{2,2}(\xi, \eta, \zeta) m_{2}(\eta, \zeta)$.}

A straightforward calculation shows that

$$
\left|\partial_{\xi_{1}}^{\alpha_{1}} \partial_{\xi_{2}}^{\alpha_{2}} \partial_{\eta_{1}}^{\beta_{1}} \partial_{\eta_{2}}^{\beta_{2}} \partial_{\zeta_{1}}^{\gamma_{1}} \partial_{\zeta_{2}}^{\gamma_{2}}\left(m_{1}^{2,2}(\xi, \eta, \zeta) m_{2}(\eta, \zeta)\right)\right| \lesssim \frac{\left(\left|\eta_{1}\right|+\left|\zeta_{1}\right|\right)^{N-\beta_{1}-\gamma_{1}}}{\left|\xi_{1}\right|^{N+\alpha_{1}}} \frac{\left(\left|\eta_{2}\right|+\left|\zeta_{2}\right|\right)^{N-\beta_{2}-\gamma_{2}}}{\left|\xi_{2}\right|^{N+\alpha_{2}}}
$$

which means that

$$
\begin{aligned}
& \left|\partial_{\xi_{1}}^{\alpha_{1}} \partial_{\xi_{2}}^{\alpha_{2}} \partial_{\eta_{1}}^{\beta_{1}} \partial_{\eta_{2}}^{\beta_{2}} \partial_{\zeta_{1}}^{\gamma_{1}} \partial_{\zeta_{2}}^{\gamma_{2}}\left(m_{1}^{2,2}(\xi, \eta, \zeta) m_{2}(\eta, \zeta) \chi\left(\xi_{1}, \eta_{1}, \zeta_{1}\right) \chi\left(\xi_{2}, \eta_{2}, \zeta_{2}\right)\right)\right| \\
& \lesssim \frac{1}{\left(\left|\xi_{1}\right|+\left|\eta_{1}\right|+\left|\zeta_{1}\right|\right)^{\alpha_{1}+\beta_{1}+\gamma_{1}}} \frac{1}{\left(\left|\xi_{2}\right|+\left|\eta_{2}\right|+\left|\zeta_{2}\right|\right)^{\alpha_{2}+\beta_{2}+\gamma_{2}}}
\end{aligned}
$$

for $\beta_{1}+\gamma_{1} \leq N$ and $\beta_{2}+\gamma_{2} \leq N$ for $N$ sufficiently large. Therefore this symbol also falls within the scope of Theorem 1.2 .

\subsubsection{The symbol $m_{1}^{1,2}(\xi, \eta, \zeta) m_{2}(\eta, \zeta), m_{1}^{2,1}(\xi, \eta, \zeta) m_{2}(\eta, \zeta)$.}

As in the argument for $m_{1}^{1,1}(\xi, \eta, \zeta)$, by a direct calculation one can see that $m_{1}^{1,2}(\xi, \eta, \zeta) m_{2}(\eta, \zeta)$ and $m_{1}^{2,1}(\xi, \eta, \zeta) m_{2}(\eta, \zeta)$ satisfy the classical restriction on the second and first parameter respectively. More precisely, for example,

$$
\lesssim \sum_{\substack{\beta_{1}^{\prime}+\beta_{1}^{\prime \prime}=\beta_{1} \\ \gamma_{1}^{\prime}+\gamma_{1}^{\prime \prime}=\gamma_{1}}} \frac{\left|\partial_{\xi_{1}}^{\alpha_{1}} \partial_{\xi_{2}}^{\alpha_{2}} \partial_{\eta_{1}}^{\beta_{1}} \partial_{\eta_{2}}^{\beta_{2}} \partial_{\zeta_{1}}^{\gamma_{1}} \partial_{\zeta_{2}}^{\gamma_{2}}\left(m_{1}^{1,2}(\xi, \eta, \zeta) m_{2}(\eta, \zeta)\right)\right|}{1} \frac{1}{\left(\left|\xi_{1}\right|+\left|\eta_{1}\right|+\left|\zeta_{1}\right|\right)^{\alpha_{1}+\beta_{1}^{\prime}+\gamma_{1}^{\prime}}} \frac{1}{\left(\left|\eta_{1}\right|+\left|\zeta_{1}\right|\right)^{\beta_{1}^{\prime \prime}+\gamma_{1}^{\prime \prime}}} \frac{1}{\left(\left|\xi_{2}\right|+\left|\eta_{2}\right|+\left|\zeta_{2}\right|\right)^{\alpha_{2}+\beta_{2}+\gamma_{2}}},
$$


for $\beta_{2}+\gamma_{2} \leq N$, where $N$ sufficiently large. Note that this is stronger than the condition that $m_{1}^{1,1}(\xi, \eta, \zeta) m_{2}(\eta, \zeta)$ satisfies, i.e.,

$$
\begin{gathered}
\lesssim \sum_{\substack{\beta_{1}^{\prime}+\beta_{1}^{\prime \prime}=\beta_{1} \\
\gamma_{1}^{\prime}+\gamma_{1}^{\prime \prime}=\gamma_{1}}} \sum_{\substack{\beta_{2}^{\prime}+\beta_{2}^{\prime \prime}=\gamma_{2}=\gamma_{2} \\
\gamma_{\xi_{1}}^{\prime}}}^{\partial_{\xi_{2}}^{\alpha_{1}} \partial_{\eta_{1}}^{\alpha_{2}} \partial_{\eta_{2}}^{\beta_{1}} \partial_{\eta_{2}}^{\beta_{2}} \partial_{\zeta_{1}}^{\gamma_{1}} \partial_{\zeta_{2}}^{\gamma_{2}}\left(m_{1}^{1,1}(\xi, \eta, \zeta) m_{2}(\eta, \zeta)\right) \mid} \frac{1}{\left(\left|\xi_{1}\right|+\left|\eta_{1}\right|+\left|\zeta_{1}\right|\right)^{\alpha_{1}+\beta_{1}^{\prime}+\gamma_{1}^{\prime}}} \frac{1}{\left(\left|\eta_{1}\right|+\left|\zeta_{1}\right|\right)^{\beta_{1}^{\prime \prime}+\gamma_{1}^{\prime \prime}}} \\
\cdot \frac{1}{\left(\left|\xi_{2}\right|+\left|\eta_{2}\right|+\left|\zeta_{2}\right|\right)^{\alpha_{2}+\beta_{2}^{\prime}+\gamma_{2}^{\prime}}} \frac{1}{\left(\left|\eta_{2}\right|+\left|\zeta_{2}\right|\right)^{\beta_{2}^{\prime \prime}+\gamma_{2}^{\prime \prime}}} .
\end{gathered}
$$

Thus, we only need to consider the symbol $m_{1}^{1,1}(\xi, \eta, \zeta) m_{2}(\eta, \zeta)$.

4.1.3. The symbol $m_{1}^{1,1}(\xi, \eta, \zeta) m_{2}(\eta, \zeta)$.

To handle the symbol $m_{1}^{1,1}(\xi, \eta, \zeta) m_{2}(\eta, \zeta)$, we use the standard decomposition for $m_{2}(\eta, \zeta)$. We denote by $\widehat{\psi}^{\prime}(u):=\sum_{k=-2}^{2} \widehat{\psi}\left(2^{-k} u\right)$, so that $\widehat{\psi}^{\prime} \in \mathcal{S}(\mathbb{R})$ and $\operatorname{supp} \widehat{\psi}^{\prime} \subset$ $\left\{u \in \mathbb{R}: 2^{-3} \leq|u| \leq 2^{3}\right\}$. Then we can write

$$
\begin{aligned}
1= & \sum_{k_{1}, k_{1}{ }^{\prime} \in \mathbb{Z}} \sum_{k_{2}, k_{2}{ }^{\prime} \in \mathbb{Z}} \widehat{\psi}\left(2^{-k_{1}} \eta_{1}\right) \widehat{\psi}\left(2^{-k_{1}^{\prime}} \zeta_{1}\right) \widehat{\psi}\left(2^{-k_{2}} \eta_{2}\right) \widehat{\psi}\left(2^{-k_{2}{ }^{\prime}} \zeta_{2}\right) \\
= & \left(\sum_{\left|k_{1}-k_{1}{ }^{\prime}\right| \leq 2}+\sum_{k_{1}-k_{1}{ }^{\prime}>2}+\sum_{k_{1}-k_{1}{ }^{\prime}<-2}\right)\left(\sum_{\left|k_{2}-k_{2}{ }^{\prime}\right| \leq 2}+\sum_{k_{2}-k_{2}{ }^{\prime}>2}+\sum_{k_{2}-k_{2}{ }^{\prime}<-2}\right) \\
& \left.\sum_{k_{1}, k_{2} \in \mathbb{Z}}\left(2^{-k_{1}} \eta_{1}\right) \widehat{\psi}\left(2^{-k_{1}^{\prime}} \zeta_{1}\right) \widehat{\psi}\left(2^{-k_{1}} \eta_{1}\right) \widehat{\psi}^{\prime}\left(2^{-k_{1}} \eta_{2}\right) \widehat{\psi}\left(2^{-k_{2}{ }^{\prime}} \zeta_{2}\right)+\widehat{\psi}\left(2^{-k_{1}} \eta_{1}\right) \widehat{\varphi}\left(2^{-k_{1}} \zeta_{1}\right)+\widehat{\varphi}\left(2^{-k_{1}} \eta_{1}\right) \widehat{\psi}\left(2^{-k_{1}} \zeta_{1}\right)\right) \\
& \cdot\left(\widehat{\psi}\left(2^{-k_{2}} \eta_{2}\right) \widehat{\psi}^{\prime}\left(2^{-k_{2}} \zeta_{2}\right)+\widehat{\psi}\left(2^{-k_{2}} \eta_{2}\right) \widehat{\varphi}\left(2^{-k_{2}} \zeta_{2}\right)+\widehat{\varphi}\left(2^{-k_{2}} \eta_{2}\right) \widehat{\psi}\left(2^{-k_{2}} \zeta_{2}\right)\right)
\end{aligned}
$$

Applying the above decomposition to $m_{2}(\eta, \zeta)$, by symmetry it suffices to consider the following cases:

$$
\begin{aligned}
m_{2}^{1,1}(\eta, \zeta) & =\sum_{k_{1}, k_{2} \in \mathbb{Z}} m_{2}(\eta, \zeta) \widehat{\psi}\left(2^{-k_{1}} \eta_{1}\right) \widehat{\psi}^{\prime}\left(2^{-k_{1}} \zeta_{1}\right) \widehat{\psi}\left(2^{-k_{2}} \eta_{2}\right) \widehat{\psi}^{\prime}\left(2^{-k_{2}} \zeta_{2}\right) \\
m_{2}^{1,2}(\eta, \zeta) & =\sum_{k_{1}, k_{2} \in \mathbb{Z}} m_{2}(\eta, \zeta) \widehat{\psi}\left(2^{-k_{1}} \eta_{1}\right) \widehat{\psi}\left(2^{-k_{1}} \zeta_{1}\right) \widehat{\psi}\left(2^{-k_{2}} \eta_{2}\right) \widehat{\varphi}\left(2^{-k_{2}} \zeta_{2}\right) \\
m_{2}^{2,2}(\eta, \zeta) & =\sum_{k_{1}, k_{2} \in \mathbb{Z}} m_{2}(\eta, \zeta) \widehat{\psi}\left(2^{-k_{1}} \eta_{1}\right) \widehat{\varphi}\left(2^{-k_{1}} \zeta_{1}\right) \widehat{\psi}\left(2^{-k_{2}} \eta_{2}\right) \widehat{\varphi}\left(2^{-k_{2}} \zeta_{2}\right) \\
m_{2}^{2,3}(\eta, \zeta) & =\sum_{k_{1}, k_{2} \in \mathbb{Z}} m_{2}(\eta, \zeta) \widehat{\psi}\left(2^{-k_{1}} \eta_{1}\right) \widehat{\varphi}\left(2^{-k_{1}} \zeta_{1}\right) \widehat{\varphi}\left(2^{-k_{2}} \eta_{2}\right) \widehat{\psi}\left(2^{-k_{2}} \zeta_{2}\right) .
\end{aligned}
$$


We now rewrite these using their Fourier expansions. For example,

$$
\begin{gathered}
m_{2}^{1,1}(\eta, \zeta)=\sum_{k_{1}, k_{2} \in \mathbb{Z}} \sum_{n_{1}, n_{2} \in \mathbb{Z}} \sum_{n_{1}^{\prime}, n_{2}^{\prime} \in \mathbb{Z}} c_{k_{1}, k_{2}, n_{1}, n_{1}^{\prime}, n_{2}, n_{2}^{\prime}}^{1,1} e^{i n_{1} \frac{\eta_{1}}{2^{k_{1}}} e^{i \frac{n_{2}}{4} \frac{\zeta_{1}}{2^{k_{1}}}} e^{i n_{1}^{\prime} \frac{\eta_{2}}{2^{k_{2}}} e^{i \frac{n_{2}^{\prime}}{4} \frac{\zeta_{2}}{2^{k_{2}}}}}} \\
\cdot \widehat{\psi}_{k_{1}}\left(\eta_{1}\right) \widehat{\psi}_{k_{1}}^{\prime}\left(\zeta_{1}\right) \widehat{\psi}_{k_{2}}\left(\eta_{2}\right) \widehat{\psi}_{k_{2}}^{\prime}\left(\zeta_{2}\right),
\end{gathered}
$$

where

$$
\sup _{k_{1}, k_{2} \in \mathbb{Z}}\left|c_{k_{1}, k_{2}, n_{1}, n_{1}^{\prime}, n_{2}, n_{2}^{\prime}}^{1,1}\right| \lesssim\left(1+\left|n_{1}\right|+\left|n_{2}\right|\right)^{-M}\left(1+\left|n_{1}^{\prime}\right|+\left|n_{2}^{\prime}\right|\right)^{-M}
$$

for sufficiently large $M>0$.

Moreover,for any index $\beta \in \mathbb{N}$ and $n \in \mathbb{Z}$ we denote by

$$
\widehat{\psi}_{\beta, n}(u)=u^{\beta} e^{i n u} \widehat{\psi}(u), \widehat{\psi}_{\beta, n}^{\prime}(u)=u^{\beta} e^{i \frac{n}{4} u} \widehat{\psi}^{\prime}(u), \widehat{\varphi}_{\beta, n}(u)=u^{\beta} e^{i n u} \widehat{\varphi}(u) .
$$

Then we have

$$
\begin{aligned}
& \eta_{1}^{\beta_{1}^{\prime}} \eta_{2}^{\beta_{2}^{\prime}} \zeta_{1}^{\gamma_{1}^{\prime}} \zeta_{2}^{\gamma_{2}^{\prime}} m_{2}^{1,1}(\eta, \zeta)=\sum_{k_{1}, k_{2} \in \mathbb{Z}} \sum_{n_{1}, n_{2} \in \mathbb{Z}} \sum_{n_{1}^{\prime}, n_{2}^{\prime} \in \mathbb{Z}} c_{k_{1}, k_{2}, n_{1}, n_{1}^{\prime}, n_{2}, n_{2}^{\prime}}^{1,1} 2^{k_{1}\left(\beta_{1}^{\prime}+\gamma_{1}^{\prime}\right)} 2^{k_{2}\left(\beta_{2}^{\prime}+\gamma_{2}^{\prime}\right)} \\
& \cdot \widehat{\psi}_{\beta_{1}^{\prime}, n_{1}}\left(2^{-k_{1}} \eta_{1}\right) \widehat{\psi}_{\gamma_{1}^{\prime}, n_{2}}^{\prime}\left(2^{-k_{1}} \zeta_{1}\right) \widehat{\psi}_{\beta_{2}^{\prime}, n_{1}^{\prime}}\left(2^{-k_{2}} \eta_{2}\right) \widehat{\psi}_{\gamma_{2}^{\prime}, n_{2}^{\prime}}^{\prime}\left(2^{-k_{2}} \zeta_{2}\right) \\
& \eta_{1}^{\beta_{1}^{\prime}} \eta_{2}^{\beta_{2}^{\prime}} \zeta_{1}^{\gamma_{1}^{\prime}} \zeta_{2}^{\gamma_{2}^{\prime}} m_{2}^{1,2}(\eta, \zeta)=\sum_{k_{1}, k_{2} \in \mathbb{Z}} \sum_{n_{1}, n_{2} \in \mathbb{Z}} \sum_{n_{1}^{\prime}, n_{2}^{\prime} \in \mathbb{Z}} c_{k_{1}, k_{2}, n_{1}, n_{1}^{\prime}, n_{2}, n_{2}^{\prime}}^{1,2} 2^{k_{1}\left(\beta_{1}^{\prime}+\gamma_{1}^{\prime}\right)} 2^{k_{2}\left(\beta_{2}^{\prime}+\gamma_{2}^{\prime}\right)} \\
& \cdot \widehat{\psi}_{\beta_{1}^{\prime}, n_{1}}\left(2^{-k_{1}} \eta_{1}\right) \widehat{\psi}_{\gamma_{1}^{\prime}, n_{2}}^{\prime}\left(2^{-k_{1}} \zeta_{1}\right) \widehat{\psi}_{\beta_{2}^{\prime}, n_{1}^{\prime}}\left(2^{-k_{2}} \eta_{2}\right) \widehat{\varphi}_{\gamma_{2}^{\prime}, n_{2}^{\prime}}\left(2^{-k_{2}} \zeta_{2}\right) \\
& \eta_{1}^{\beta_{1}^{\prime}} \eta_{2}^{\beta_{2}^{\prime}} \zeta_{1}^{\gamma_{1}^{\prime}} \zeta_{2}^{\gamma_{2}^{\prime}} m_{2}^{2,2}(\eta, \zeta)=\sum_{k_{1}, k_{2} \in \mathbb{Z}} \sum_{n_{1}, n_{2} \in \mathbb{Z}} \sum_{n_{1}^{\prime}, n_{2}^{\prime} \in \mathbb{Z}} c_{k_{1}, k_{2}, n_{1}, n_{1}^{\prime}, n_{2}, n_{2}^{\prime}}^{2,} 2^{k_{1}\left(\beta_{1}^{\prime}+\gamma_{1}^{\prime}\right)} 2^{k_{2}\left(\beta_{2}^{\prime}+\gamma_{2}^{\prime}\right)} \\
& \cdot \widehat{\psi}_{\beta_{1}^{\prime}, n_{1}}\left(2^{-k_{1}} \eta_{1}\right) \widehat{\varphi}_{\gamma_{1}^{\prime}, n_{2}}\left(2^{-k_{1}} \zeta_{1}\right) \widehat{\psi}_{\beta_{2}^{\prime}, n_{1}^{\prime}}\left(2^{-k_{2}} \eta_{2}\right) \widehat{\varphi}_{\gamma_{2}^{\prime}, n_{2}^{\prime}}\left(2^{-k_{2}} \zeta_{2}\right) \\
& \eta_{1}^{\beta_{1}^{\prime}} \eta_{2}^{\beta_{2}^{\prime}} \zeta_{1}^{\gamma_{1}^{\prime}} \zeta_{2}^{\gamma_{2}^{\prime}} m_{2}^{2,3}(\eta, \zeta)=\sum_{k_{1}, k_{2} \in \mathbb{Z}} \sum_{n_{1}, n_{2} \in \mathbb{Z}} \sum_{n_{1}^{\prime}, n_{2}^{\prime} \in \mathbb{Z}} c_{k_{1}, k_{2}, n_{1}, n_{1}^{\prime}, n_{2}, n_{2}^{\prime}}^{2,3} 2^{k_{1}\left(\beta_{1}^{\prime}+\gamma_{1}^{\prime}\right)} 2^{k_{2}\left(\beta_{2}^{\prime}+\gamma_{2}^{\prime}\right)} \\
& \cdot \widehat{\psi}_{\beta_{1}^{\prime}, n_{1}}\left(2^{-k_{1}} \eta_{1}\right) \widehat{\varphi}_{\gamma_{1}^{\prime}, n_{2}}\left(2^{-k_{1}} \zeta_{1}\right) \widehat{\varphi}_{\beta_{2}^{\prime}, n_{1}^{\prime}}\left(2^{-k_{2}} \eta_{2}\right) \widehat{\psi}_{\gamma_{2}^{\prime}, n_{2}^{\prime}}\left(2^{-k_{2}} \zeta_{2}\right) \text {, }
\end{aligned}
$$

where the coefficients, for all four pairs $\left(i_{1}, i_{2}\right)$ above, satisfy $\sup _{k_{1}, k_{2} \in \mathbb{Z}}\left|c_{k_{1}, k_{2}, n_{1}, n_{1}^{\prime}, n_{2}, n_{2}^{\prime}}^{i_{1}, i_{2}}\right| \lesssim$ $\left(1+\left|n_{1}\right|+\left|n_{2}\right|\right)^{-M}\left(1+\left|n_{1}^{\prime}\right|+\left|n_{2}^{\prime}\right|\right)^{-M}$.

In similar fashion, for $m_{1}^{1,1}(\xi, \eta, \zeta)$ in (4.4), we denote

$$
M_{\beta_{1}^{\prime}, \beta_{2}^{\prime}, \gamma_{1}^{\prime}, \gamma_{2}^{\prime}}(\xi):=\frac{1}{\beta_{1}^{\prime} ! \beta_{2}^{\prime} ! \gamma_{1}^{\prime} ! \gamma_{2}^{\prime} !} \partial_{\eta_{1}}^{\beta_{1}^{\prime}} \partial_{\eta_{2}}^{\beta_{2}^{\prime}} \partial_{\zeta_{1}}^{\gamma_{1}^{\prime}} \partial_{\zeta_{2}}^{\gamma_{2}^{\prime}} m_{1}(\xi, 0,0,0,0) \text {. }
$$

Note that for any indices $\alpha_{1}, \alpha_{2}$ there holds

$$
\left|\partial_{\xi_{1}}^{\alpha_{1}} \partial_{\xi_{2}}^{\alpha_{2}} M_{\beta_{1}^{\prime}, \beta_{2}^{\prime}, \gamma_{1}^{\prime}, \gamma_{2}^{\prime}}(\xi)\right| \lesssim\left|\xi_{1}\right|^{-\left(\alpha_{1}+\beta_{1}^{\prime}+\gamma_{1}^{\prime}\right)}\left|\xi_{2}\right|^{-\left(\alpha_{2}+\beta_{2}^{\prime}+\gamma_{2}^{\prime}\right)}
$$


which means we can expand in Fourier series to write as:

$$
\begin{gathered}
M_{\beta_{1}^{\prime}, \beta_{2}^{\prime}, \gamma_{1}^{\prime}, \gamma_{2}^{\prime}}(\xi) \widehat{\psi}\left(2^{-j_{1}} \xi_{1}\right) \widehat{\psi}\left(2^{-j_{2}} \xi_{2}\right) \\
=\sum_{l_{1}, l_{2} \in \mathbb{Z}} c_{j_{1}, j_{2}, l_{1}, l_{2}}^{\beta_{1}^{\prime}, \beta_{2}^{\prime}, \gamma_{2}^{\prime}, \gamma_{2}^{\prime}} 2^{-j_{1}\left(\beta_{1}^{\prime}+\gamma_{1}^{\prime}\right)} 2^{-j_{2}\left(\beta_{2}^{\prime}+\gamma_{2}^{\prime}\right)} \widehat{\psi}_{0, l_{1}}\left(2^{-j_{1}} \xi_{1}\right) \widehat{\psi}_{0, l_{2}}\left(2^{-j_{2}} \xi_{2}\right),
\end{gathered}
$$

where $\widehat{\psi}_{0, l_{1}}(u)=e^{i n u} \widehat{\psi}(u), \widehat{\psi}_{0, l_{2}}(v)=e^{i n u} \widehat{\psi}(v)$ for $u, v \in \mathbb{R}$ are defined as before, and

$$
\sup _{j_{1}, j_{2}}\left|c_{j_{1}, j_{2}, l_{1}, l_{2}}^{\beta_{1}, \beta_{2}, \gamma_{1}, \gamma_{2}}\right| \lesssim\left(1+\left|l_{1}\right|\right)^{-M}\left(1+\left|l_{2}\right|\right)^{-M} .
$$

Then if we denote by $d_{1}=\beta_{1}^{\prime}+\gamma_{1}^{\prime}$ and $d_{2}=\beta_{2}^{\prime}+\gamma_{2}^{\prime}$, and put everything together, we have that

$$
\begin{aligned}
& m_{1}^{1,1}(\xi, \eta, \zeta) m_{2}(\eta, \zeta) \chi\left(\xi_{1}, \eta_{1}, \zeta_{1}\right) \chi\left(\xi_{2}, \eta_{2}, \zeta_{2}\right) \\
& =\sum c^{1,1} 2^{-\left(j_{1}-k_{1}\right) d_{1}} 2^{-\left(j_{2}-k_{2}\right) d_{2}} \widehat{\psi}_{0, l_{1}}\left(\frac{\xi_{1}}{2^{j_{1}}}\right) \widehat{\psi}_{0, l_{2}}\left(\frac{\xi_{2}}{2^{j_{2}}}\right) \widehat{\varphi}\left(\frac{\eta_{1}}{2^{j_{1}-10}}\right) \widehat{\varphi}\left(\frac{\eta_{2}}{2^{j_{2}-10}}\right) \\
& \widehat{\varphi}\left(\frac{\zeta_{1}}{2^{j_{1}-10}}\right) \widehat{\varphi}\left(\frac{\zeta_{2}}{2^{j_{2}-10}}\right) \widehat{\psi}_{\beta_{1}^{\prime}, n_{1}}\left(\frac{\eta_{1}}{2^{k_{1}}}\right) \widehat{\psi}_{\gamma_{1}^{\prime}, n_{2}}^{\prime}\left(\frac{\zeta_{1}}{2^{k_{1}}}\right) \widehat{\psi}_{\beta_{2}^{\prime}, n_{1}^{\prime}}\left(\frac{\eta_{2}}{2^{k_{2}}}\right) \widehat{\psi}_{\gamma_{2}^{\prime}, n_{2}^{\prime}}^{\prime}\left(\frac{\zeta_{2}}{2^{k_{2}}}\right) \\
& +\sum c^{1,2} 2^{-\left(j_{1}-k_{1}\right) d_{1}} 2^{-\left(j_{2}-k_{2}\right) d_{2}} \widehat{\psi}_{0, l_{1}}\left(\frac{\xi_{1}}{2^{j_{1}}}\right) \widehat{\psi}_{0, l_{2}}\left(\frac{\xi_{2}}{2^{j_{2}}}\right) \widehat{\varphi}\left(\frac{\eta_{1}}{2^{j_{1}-10}}\right) \widehat{\varphi}\left(\frac{\eta_{2}}{2^{j_{2}}-10}\right) \\
& \widehat{\varphi}\left(\frac{\zeta_{1}}{2^{j_{1}-10}}\right) \widehat{\varphi}\left(\frac{\zeta_{2}}{2^{j_{2}-10}}\right) \widehat{\psi}_{\beta_{1}^{\prime}, n_{1}}\left(\frac{\eta_{1}}{2^{k_{1}}}\right) \widehat{\psi}_{\gamma_{1}^{\prime}, n_{2}}^{\prime}\left(\frac{\zeta_{1}}{2^{k_{1}}}\right) \widehat{\psi}_{\beta_{2}^{\prime}, n_{1}^{\prime}}\left(\frac{\eta_{2}}{2^{k_{2}}}\right) \widehat{\varphi}_{\gamma_{2}^{\prime}, n_{2}^{\prime}}\left(\frac{\zeta_{2}}{2^{k_{2}}}\right) \\
& +\sum c^{2,2} 2^{-\left(j_{1}-k_{1}\right) d_{1}} 2^{-\left(j_{2}-k_{2}\right) d_{2}} \widehat{\psi}_{0, l_{1}}\left(\frac{\xi_{1}}{2^{j_{1}}}\right) \widehat{\psi}_{0, l_{2}}\left(\frac{\xi_{2}}{2^{j_{2}}}\right) \widehat{\varphi}\left(\frac{\eta_{1}}{2^{j_{1}}-10}\right) \widehat{\varphi}\left(\frac{\eta_{2}}{2^{j_{2}}-10}\right) \\
& \widehat{\varphi}\left(\frac{\zeta_{1}}{2^{j_{1}-10}}\right) \widehat{\varphi}\left(\frac{\zeta_{2}}{2^{j_{2}-10}}\right) \widehat{\psi}_{\beta_{1}^{\prime}, n_{1}}\left(\frac{\eta_{1}}{2^{k_{1}}}\right) \widehat{\varphi}_{\gamma_{1}^{\prime}, n_{2}}\left(\frac{\zeta_{1}}{2^{k_{1}}}\right) \widehat{\psi}_{\beta_{2}^{\prime}, n_{1}^{\prime}}\left(\frac{\eta_{2}}{2^{k_{2}}}\right) \widehat{\varphi}_{\gamma_{2}^{\prime}, n_{2}^{\prime}}\left(\frac{\zeta_{2}}{2^{k_{2}}}\right) \\
& +\sum c^{2,3} 2^{-\left(j_{1}-k_{1}\right) d_{1}} 2^{-\left(j_{2}-k_{2}\right) d_{2}} \widehat{\psi}_{0, l_{1}}\left(\frac{\xi_{1}}{2^{j_{1}}}\right) \widehat{\psi}_{0, l_{2}}\left(\frac{\xi_{2}}{2^{j_{2}}}\right) \widehat{\varphi}\left(\frac{\eta_{1}}{2^{j_{1}}-10}\right) \widehat{\varphi}\left(\frac{\eta_{2}}{2^{j_{2}}-10}\right) \\
& \widehat{\varphi}\left(\frac{\zeta_{1}}{2^{j_{1}-10}}\right) \widehat{\varphi}\left(\frac{\zeta_{2}}{2^{j_{2}-10}}\right) \widehat{\psi}_{\beta_{1}^{\prime}, n_{1}}\left(\frac{\eta_{1}}{2^{k_{1}}}\right) \widehat{\varphi}_{\gamma_{1}^{\prime}, n_{2}}\left(\frac{\zeta_{1}}{2^{k_{1}}}\right) \widehat{\varphi}_{\beta_{2}^{\prime}, n_{1}^{\prime}}\left(\frac{\eta_{2}}{2^{k_{2}}}\right) \widehat{\psi}_{\gamma_{2}^{\prime}, n_{2}^{\prime}}^{\prime}\left(\frac{\zeta_{2}}{2^{k_{2}}}\right),
\end{aligned}
$$

where the summation is taken over $j_{1}, j_{2}, k_{1}, k_{2}, l_{1}, l_{2}, n_{1}, n_{2}, n_{1}^{\prime}, n_{2}^{\prime}$ and

$$
c^{i_{1}, i_{2}}=c_{j_{1}, j_{2}, l_{1}, l_{2}}^{\beta_{1}^{\prime}, \beta_{2}^{\prime}, \gamma_{1}^{\prime}} c_{k_{1}, k_{2}, n_{1}, n_{1}^{\prime}, n_{2}, n_{2}^{\prime}}^{i_{1}, i_{2}}
$$

for all the four pairs $\left(i_{1}, i_{2}\right)$ as above.

Note that when $j_{1}-k_{1}<10$ or $j_{2}-k_{2}<10$, each of the four parts in (4.6) must be zero. Actually, if we take a look at the expressions in (4.6), for example,

$$
\begin{gathered}
\sum c^{2,3} 2^{-\left(j_{1}-k_{1}\right) d_{1}} 2^{-\left(j_{2}-k_{2}\right) d_{2}} \widehat{\psi}_{0, l_{1}}\left(\frac{\xi_{1}}{2^{j_{1}}}\right) \widehat{\psi}_{0, l_{2}}\left(\frac{\xi_{2}}{2^{j_{2}}}\right) \widehat{\varphi}\left(\frac{\eta_{1}}{2^{j_{1}-10}}\right) \widehat{\varphi}\left(\frac{\eta_{2}}{2^{j_{2}-10}}\right) \\
\cdot \widehat{\varphi}\left(\frac{\zeta_{1}}{2^{j_{1}-10}}\right) \widehat{\varphi}\left(\frac{\zeta_{2}}{2^{j_{2}-10}}\right) \widehat{\psi}_{\beta_{1}^{\prime}, n_{1}}\left(\frac{\eta_{1}}{2^{k_{1}}}\right) \widehat{\varphi}_{\gamma_{1}^{\prime}, n_{2}}\left(\frac{\zeta_{1}}{2^{k_{1}}}\right) \widehat{\varphi}_{\beta_{2}^{\prime}, n_{1}^{\prime}}\left(\frac{\eta_{2}}{2^{k_{2}}}\right) \widehat{\psi}_{\gamma_{2}^{\prime}, n_{2}^{\prime}}^{\prime}\left(\frac{\zeta_{2}}{2^{k_{2}}}\right),
\end{gathered}
$$


we can see actually $\widehat{\varphi}\left(\frac{\eta_{1}}{2^{j_{1}}-10}\right) \widehat{\psi}_{\beta_{1}^{\prime}, n_{1}}\left(\frac{\eta_{1}}{2^{k_{1}}}\right)=0$, since they have disjoint supports. More precisely,

$$
\begin{gathered}
\operatorname{supp} \widehat{\varphi}\left(\frac{\eta_{1}}{2^{j_{1}-10}}\right) \subset\left\{\eta_{1}:\left|\eta_{1}\right| \leq 2^{j_{1}-12}\right\}, \\
\widehat{\psi}_{\beta_{1}^{\prime}, n_{1}}\left(\frac{\eta_{1}}{2^{k_{1}}}\right) \subset\left\{\eta_{1}: 2^{k_{1}-1} \leq\left|\eta_{1}\right| \leq 2^{k_{1}+1}\right\}, \\
\operatorname{supp} \widehat{\varphi}\left(\frac{\eta_{1}}{2^{j_{1}-10}}\right) \cap \operatorname{supp} \widehat{\psi}_{\beta_{1}^{\prime}, n_{1}}\left(\frac{\eta_{1}}{2^{k_{1}}}\right)=\emptyset \quad \text { if } \quad j_{1}-k_{1}<10 .
\end{gathered}
$$

Other terms are handled similarly.

Therefore, we just need to consider the case $j_{1}-k_{1} \geq 10$ and $j_{2}-k_{2} \geq 10$. Moreover, when $j_{1}-k_{1} \geq 20, \widehat{\varphi}\left(\frac{\eta_{1}}{2^{j_{1}}-10}\right)=1$ on $\operatorname{supp} \widehat{\psi}_{\beta_{1}^{\prime}, n_{1}}\left(\frac{\eta_{1}}{2^{k_{1}}}\right) \cup \operatorname{supp} \widehat{\varphi}_{\beta_{1}^{\prime}, n_{1}}\left(\frac{\eta_{1}}{2^{k_{1}}}\right)$ and $\widehat{\varphi}\left(\frac{\zeta_{1}}{2^{j_{1}}-10}\right)=1$ on $\operatorname{supp} \widehat{\psi}_{\gamma_{1}^{\prime}, n_{2}}\left(\frac{\zeta_{1}}{2^{k_{1}}}\right) \cup \operatorname{supp} \widehat{\varphi}_{\gamma_{1}^{\prime}, n_{2}}\left(\frac{\zeta_{1}}{2^{k_{1}}}\right)$. Further, when $10<j_{1}-k_{1}<20$, one can see that the summation of the terms involving $\xi_{1}, \eta_{1}, \zeta_{1}$ gives a multiplier in $\mathcal{M}\left(\mathbb{R}^{3}\right)$. The same argument works for the other half of variables $\xi_{2}, \eta_{2}, \zeta_{2}$ as well based on the similar choice of $k_{2}, j_{2}$.

Due to the decay in the coefficients $a_{j_{1}, j_{2}}:=c_{j_{1}, j_{2}, l_{1}, l_{2}}^{\beta_{1}^{\prime}, \beta_{2}^{\prime}, \gamma_{2}^{\prime}, \gamma_{k_{1}, k_{2}}^{\prime}}:=c_{k_{1}, k_{2}, n_{1}, n_{1}^{\prime}, n_{2}, n_{2}^{\prime}}^{i_{1}, i_{2}}$, we can fix $l_{1}, l_{2}, n_{1}, n_{2}, n_{1}^{\prime}, n_{2}^{\prime}$ and only take the summation over $j_{1}, j_{2}, k_{1}, k_{2}$. And without loss of generality we can assume $\left|a_{j_{1}, j_{2}}\right| \leq 1,\left|b_{k_{1}, k_{2}}\right| \leq 1$.

Thus, when $10 \leq j_{1}-k_{1} \leq 20$ and $10 \leq j_{2}-k_{2} \leq 20$, the above multipliers belong to $B \mathcal{M}\left(\mathbb{R}^{6}\right)$ and Theorem 1.2 gives us the desired estimate. So we only need to consider the case $j_{1}-k_{1} \geq 20, j_{2}-k_{2} \geq 20$, and the cases $j_{1}-k_{1} \geq 20,10 \leq j_{2}-k_{2} \leq 20$ and $10 \leq j_{1}-k_{1} \leq 20, j_{2}-k_{2} \geq 20$ correspond to the estimates like (4.1) and (4.5).

For the reduction in (4.6), one will see later what really matters there is the "type" of those $\widehat{\psi}$ and $\widehat{\varphi}$ functions, i.e, whether 0 is contained in the supports of functions. We call these functions $\Psi$ type and $\Phi$ type functions respectively. Because of that, we can simplify the notations for operators in (4.6) as below, where we also denote by $d_{i}=\beta_{i}^{\prime}+\gamma_{i}^{\prime}<N$ for $i=1,2$.

$$
\begin{gathered}
m_{d_{1}, d_{2}}^{1}(\xi, \eta, \zeta)= \\
\sum_{\substack{j_{1}-k_{1} \geq 20 \\
j_{2}-k_{2} \geq 20}} a_{j_{1}, j_{2}} b_{k_{1}, k_{2}} 2^{-\left(j_{1}-k_{1}\right) d_{1}} 2^{-\left(j_{2}-k_{2}\right) d_{2}} \widehat{\psi}^{0}\left(\frac{\xi_{1}}{2^{j_{1}}}\right) \widehat{\widetilde{\psi}^{0}}\left(\frac{\xi_{2}}{2^{j_{2}}}\right) \widehat{\psi}^{1}\left(\frac{\eta_{1}}{2^{k_{1}}}\right) \widehat{\psi}^{2}\left(\frac{\zeta_{1}}{2^{k_{1}}}\right) \widehat{\widetilde{\psi}^{1}}\left(\frac{\eta_{2}}{2^{k_{2}}}\right) \widehat{\widetilde{\psi}^{2}}\left(\frac{\zeta_{2}}{2^{k_{2}}}\right), \\
m_{d_{1}, d_{2}}^{2}(\xi, \eta, \zeta)=
\end{gathered}
$$


$\sum_{\substack{j_{1}-k_{1} \geq 20 \\ j_{2}-k_{2} \geq 20}} a_{j_{1}, j_{2}} b_{k_{1}, k_{2}} 2^{-\left(j_{1}-k_{1}\right) d_{1}} 2^{-\left(j_{2}-k_{2}\right) d_{2}} \widehat{\psi}^{0}\left(\frac{\xi_{1}}{2^{j_{1}}}\right) \widehat{\widetilde{\psi}^{0}}\left(\frac{\xi_{2}}{2^{j_{2}}}\right) \widehat{\psi}^{1}\left(\frac{\eta_{1}}{2^{k_{1}}}\right) \widehat{\psi}^{2}\left(\frac{\zeta_{1}}{2^{k_{1}}}\right) \widehat{\bar{\psi}^{1}}\left(\frac{\eta_{2}}{2^{k_{2}}}\right) \widehat{\widetilde{\varphi}^{0}}\left(\frac{\zeta_{2}}{2^{k_{2}}}\right)$,

$m_{d_{1}, d_{2}}^{3}(\xi, \eta, \zeta)=$
$\sum_{\substack{j_{1}-k_{1} \geq 20 \\ j_{2}-k_{2} \geq 20}} a_{j_{1}, j_{2}} b_{k_{1}, k_{2}} 2^{-\left(j_{1}-k_{1}\right) d_{1}} 2^{-\left(j_{2}-k_{2}\right) d_{2}} \widehat{\psi}^{0}\left(\frac{\xi_{1}}{2^{j_{1}}}\right) \widehat{\widetilde{\psi}^{0}}\left(\frac{\xi_{2}}{2^{j_{2}}}\right) \widehat{\psi}^{1}\left(\frac{\eta_{1}}{2^{k_{1}}}\right) \widehat{\varphi}^{0}\left(\frac{\zeta_{1}}{2^{k_{1}}}\right) \widehat{\widetilde{\psi}^{1}}\left(\frac{\eta_{2}}{2^{k_{2}}}\right) \widehat{\widetilde{\varphi}^{0}}\left(\frac{\zeta_{2}}{2^{k_{2}}}\right)$,

$m_{d_{1}, d_{2}}^{4}(\xi, \eta, \zeta)=$
$\sum_{\substack{j_{1}-k_{1} \geq 20 \\ j_{2}-k_{2} \geq 20}} a_{j_{1}, j_{2}} b_{k_{1}, k_{2}} 2^{-\left(j_{1}-k_{1}\right) d_{1}} 2^{-\left(j_{2}-k_{2}\right) d_{2}} \widehat{\psi}^{0}\left(\frac{\xi_{1}}{2^{j_{1}}}\right) \widehat{\widetilde{\psi}^{0}}\left(\frac{\xi_{2}}{2^{j_{2}}}\right) \widehat{\psi}^{1}\left(\frac{\eta_{1}}{2^{k_{1}}}\right) \widehat{\varphi}^{0}\left(\frac{\zeta_{1}}{2^{k_{1}}}\right) \widehat{\widetilde{\varphi}^{0}}\left(\frac{\eta_{2}}{2^{k_{2}}}\right) \widehat{\widetilde{\psi}^{1}}\left(\frac{\zeta_{2}}{2^{k_{2}}}\right)$.

Here $\widehat{\psi}^{i}, \widehat{\varphi}^{0}, \widehat{\bar{\psi}^{i}}, \widehat{\widetilde{\varphi}^{0}}$ satisfy

$$
\begin{aligned}
& \operatorname{supp} \widehat{\psi}^{i}, \operatorname{supp} \widetilde{\widetilde{\psi}^{i}} \subset\left\{u\left|2^{-1} \leq\right| u \mid \leq 2\right\}, \quad i=0,1, \\
& \operatorname{supp} \widehat{\psi}^{2}, \operatorname{supp} \widehat{\widetilde{\psi}^{2}} \subset\left\{u\left|2^{-3} \leq\right| u \mid \leq 2^{3}\right\}, \\
& \operatorname{supp} \widehat{\varphi}^{0}, \operatorname{supp} \widehat{\widetilde{\varphi}^{0}} \subset\left\{u|| u \mid \leq 2^{-2}\right\} .
\end{aligned}
$$

As previously mentioned, because of the "type" of the functions, we do not distinguish between $\widehat{\psi}^{i}$ and $\widehat{\psi^{i}}(i=0,1,2)$, between $\widehat{\varphi}^{0}$ and $\widehat{\widetilde{\varphi}^{0}}$, and we denote them to be $\widehat{\psi}$ and $\widehat{\varphi}$ respectively. But note that $\widehat{\psi}, \widehat{\varphi}$ are different from the ones in (4.2) and (4.3). And we use the notations

$$
\widehat{\Delta_{j} f}(\xi)=\widehat{\psi}\left(\frac{\xi}{2^{j}}\right) \hat{f}(\xi), \quad \widehat{S_{k} f}(\xi)=\widehat{\varphi}\left(\frac{\xi}{2^{k}}\right) \hat{f}(\xi) .
$$

Then finally we reduce our original problem to the study of the following cases

$$
\begin{aligned}
T_{d_{1}, d_{2}}^{1} & =\sum_{\substack{j_{1}-k_{1} \geq 20 \\
j_{2}-k_{2} \geq 20}} a_{j_{1}, j_{2}} b_{k_{1}, k_{2}} 2^{-\left(j_{1}-k_{1}\right) d_{1}} 2^{-\left(j_{2}-k_{2}\right) d_{2}} \Delta_{j_{1}} \Delta_{j_{2}} f \Delta_{k_{1}} \Delta_{k_{2}} g \Delta_{k_{1}} \Delta_{k_{2}} h \\
T_{d_{1}, d_{2}}^{2} & =\sum_{\substack{j_{1}-k_{1} \geq 20 \\
j_{2}-k_{2} \geq 20}} a_{j_{1}, j_{2}} b_{k_{1}, k_{2}} 2^{-\left(j_{1}-k_{1}\right) d_{1}} 2^{-\left(j_{2}-k_{2}\right) d_{2}} \Delta_{j_{1}} \Delta_{j_{2}} f \Delta_{k_{1}} \Delta_{k_{2}} g \Delta_{k_{1}} S_{k_{2}} h \\
T_{d_{1}, d_{2}}^{3}= & \sum_{\substack{j_{1}-k_{1} \geq 20 \\
j_{2}-k_{2} \geq 20}} a_{j_{1}, j_{2}} b_{k_{1}, k_{2}} 2^{-\left(j_{1}-k_{1}\right) d_{1}} 2^{-\left(j_{2}-k_{2}\right) d_{2}} \Delta_{j_{1}} \Delta_{j_{2}} f \Delta_{k_{1}} \Delta_{k_{2}} g S_{k_{1}} S_{k_{2}} h
\end{aligned}
$$




$$
T_{d_{1}, d_{2}}^{4}=\sum_{\substack{j_{1}-k_{1} \geq 20 \\ j_{2}-k_{2} \geq 20}} a_{j_{1}, j_{2}} b_{k_{1}, k_{2}} 2^{-\left(j_{1}-k_{1}\right) d_{1}} 2^{-\left(j_{2}-k_{2}\right) d_{2}} \Delta_{j_{1}} \Delta_{j_{2}} f \Delta_{k_{1}} S_{k_{2}} g S_{k_{1}} \Delta_{k_{2}} h
$$

Note the fact that in $T_{d_{1}, d_{2}}^{i}(i=1,2,3,4)$, the support for each of the Fourier transforms of $\left(\Delta_{k_{1}} \Delta_{k_{2}} g \Delta_{k_{1}} S_{k_{2}} h\right)\left(x_{1}, x_{2}\right),\left(\Delta_{k_{1}} \Delta_{k_{2}} g S_{k_{1}} S_{k_{2}} h\right)\left(x_{1}, x_{2}\right),\left(\Delta_{k_{1}} S_{k_{2}} g S_{k_{1}} \Delta_{k_{2}} h\right)\left(x_{1}, x_{2}\right)$ is contained in $\left\{\left|u_{1}\right| \lesssim 2^{k_{1}},\left|u_{2}\right| \lesssim 2^{k_{2}}\right\}$. Thus the Fourier transform of $T_{d_{1}, d_{2}}^{i}(i=$ $1,2,3,4)$ is supported in $\left\{\left|u_{1}\right| \approx 2^{j_{1}},\left|u_{2}\right| \approx 2^{j_{2}}\right\}$ for fixed $j_{1}, j_{2}$. Moreover, from the argument below, one can see it suffices to consider the case for $d_{1}=d_{2}=0$ since $j_{1}-k_{1} \geq 20, j_{2}-k_{2} \geq 20$.

\subsection{The $L^{r}$ boundedness of Hölder type.}

In this subsection we study the $L^{p_{1}} \times L^{p_{2}} \times L^{p_{3}} \rightarrow L^{r}$ estimate for $1<p_{1}, p_{2}, p_{3}<\infty$ for the operators $T_{d_{1}, d_{2}}^{i}(i=1,2,3,4)$.

4.2.1. $d_{1}, d_{2}>0$.

The approach for the case $d_{1}, d_{2}>0$ works for all $T_{d_{1}, d_{2}}^{i},(1 \leq i \leq 4)$. Consider $T_{d_{1}, d_{2}}^{2}$ for example, with other cases treated similarly. Since the support of the Fourier transform of $T_{d_{1}, d_{2}}^{2}$ for fixed $j_{1}, j_{2}$ is included in $\left\{\left|u_{1}\right| \approx 2^{j_{1}},\left|u_{2}\right| \approx 2^{j_{2}}\right\}$, there holds

$$
\begin{aligned}
& \left\|T_{d_{1}, d_{2}}^{2}(f, g, h)\right\|_{L^{r}} \lesssim \|\left\{\sum_{j_{1}, j_{2} \in \mathbb{Z}} \mid a_{j_{1}, j_{2}} \Delta_{j_{1}} \Delta_{j_{2}} f\left(\sum_{k_{1}=-\infty}^{j_{1}-20} \sum_{k_{2}=-\infty}^{j_{2}-20} b_{k_{1}, k_{2}} 2^{-\left(j_{1}-k_{1}\right) d_{1}} 2^{-\left(j_{2}-k_{2}\right) d_{2}}\right.\right. \\
& \left.\left.\Delta_{k_{1}} \Delta_{k_{2}} g \Delta_{k_{1}} S_{k_{2}} h\right)\left.\right|^{2}\right\}^{\frac{1}{2}} \|_{L^{r}} \\
& \lesssim\left\|\left\{\sum_{j_{1}, j_{2} \in \mathbb{Z}}\left|a_{j_{1}, j_{2}} \Delta_{j_{1}} \Delta_{j_{2}} f\right|^{2}\right\}^{\frac{1}{2}}\left(\sup _{k_{1}, k_{2}}\left|\Delta_{k_{1}} \Delta_{k_{2}} g\right|\right)\left(\sup _{k_{1}, k_{2}}\left|\Delta_{k_{1}} S_{k_{2}} h\right|\right)\right\|_{L^{r}} \\
& \lesssim\left\|\left\{\sum_{j_{1}, j_{2} \in \mathbb{Z}}\left|a_{j_{1}, j_{2}} \Delta_{j_{1}} \Delta_{j_{2}} f\right|^{2}\right\}^{\frac{1}{2}}\right\|_{L^{p_{1}}}\left\|\sup _{k_{1}, k_{2}}\left|\Delta_{k_{1}} \Delta_{k_{2}} g\right|\right\|_{L^{p_{2}}}\left\|\sup _{k_{1}, k_{2}}\left|\Delta_{k_{1}} S_{k_{2}} h\right|\right\|_{L^{p_{3}}} \\
& \lesssim\left\|\left\{\sum_{j_{1}, j_{2} \in \mathbb{Z}}\left|\Delta_{j_{1}} \Delta_{j_{2}} f\right|^{2}\right\}^{\frac{1}{2}}\right\|_{L^{p_{1}}}\left\|M_{s} g\right\|_{L^{p_{2}}}\left\|M_{s} h\right\|_{L^{p_{3}}} \\
& \lesssim\|f\|_{L^{p_{1}}}\|g\|_{L^{p_{2}}}\|h\|_{L^{p_{3}}}
\end{aligned}
$$

where $0<1 / r=1 / p_{1}+1 / p_{2}+1 / p_{3}$ with $1<p_{1}, p_{2}, p_{3}<\infty, M_{s}$ is the strong maximal operator.

4.2.2. $d_{1}=d_{2}=0$.

Note that for $d_{1}>0$ and $d_{2}=0$, or $d_{1}=0$ and $d_{2}>0$, we can treat the decay factors 
$2^{-\left(j_{1}-k_{1}\right) d_{1}}$ or $2^{-\left(j_{2}-k_{2}\right) d_{2}}$ as uniform constants. That means they are particular cases of $d_{1}=d_{2}=0$. For the case $d_{1}=d_{2}=0$, in (4.4) we have $\beta_{1}^{\prime}=\gamma_{1}^{\prime}=\beta_{2}^{\prime}=\gamma_{2}^{\prime}=0$. Here we consider $T_{d_{1}, d_{2}}^{4}$, and one can check the following argument is valid for the other three operators.

We write the the $L^{r}$ norm of $T_{0,0}^{4}$ as

$$
\begin{aligned}
& \left\|\sum_{\substack{j_{1}-k_{1} \geq 20 \\
j_{2}-k_{2} \geq 20}} a_{j_{1}, j_{2}} b_{k_{1}, k_{2}} \Delta_{j_{1}} \Delta_{j_{2}} f \Delta_{k_{1}} S_{k_{2}} g S_{k_{1}} \Delta_{k_{2}} h\right\|_{L^{r}} \\
& =\left\|\sum_{k_{1}, k_{2} \in \mathbb{Z}} b_{k_{1}, k_{2}} \Delta_{k_{1}} S_{k_{2}} g S_{k_{1}} \Delta_{k_{2}} h \sum_{j_{1}, j_{2}=k_{1}, k_{2}+20}^{\infty} a_{j_{1}, j_{2}} \Delta_{j_{1}} \Delta_{j_{2}} f\right\|_{L^{r}}
\end{aligned}
$$

We first consider the part $\sum_{j_{1}, j_{2}=k_{1}, k_{2}+20}^{\infty} \Delta_{j_{1}} \Delta_{j_{2}} f$. have

In one-parameter case, since $\operatorname{supp} \widehat{\psi}_{j} \subset\left\{2^{j-1} \leq|\xi| \leq 2^{j+1}\right\}(j \in \mathbb{Z}, \xi \in \mathbb{R})$, we

$$
\operatorname{supp} \sum_{j=k+20}^{\infty} \widehat{\psi}_{j} \subset\left\{2^{k+19} \leq|\xi|\right\} .
$$

Now we choose a function $\phi$ such that $\operatorname{supp} \widehat{\phi}_{k} \subset\left\{|\xi| \leq 2^{k-2}\right\}$ and $\widehat{\phi}_{k}=1$ on $\left\{|\xi| \leq 2^{k-4}\right\}$. Note that

$$
\begin{gathered}
1-\widehat{\phi}_{k}=1 \quad \text { on } \quad \operatorname{supp} \sum_{j=k+20}^{\infty} \widehat{\psi}_{j}, \\
1-\widehat{\phi}_{k}=0 \quad \text { on } \quad\left\{|\xi| \leq 2^{k-4}\right\} .
\end{gathered}
$$

Thus,

$$
\begin{aligned}
\sum_{j=k+20}^{\infty} \widehat{\psi}_{j} & =\left(1-\widehat{\phi}_{k}\right)\left(\sum_{j=k+20}^{\infty} \widehat{\psi}_{j}\right)=\left(1-\widehat{\phi}_{k}\right)\left(\sum_{j \in \mathbb{Z}} \widehat{\psi}_{j}-\sum_{j=-\infty}^{k+19} \widehat{\psi}_{j}\right) \\
& =\left(1-\widehat{\phi}_{k}\right)\left(\sum_{j \in \mathbb{Z}} \widehat{\psi}_{j}-\sum_{j=k-5}^{k+19} \widehat{\psi}_{j}\right)=\left(1-\widehat{\phi}_{k}\right)\left(\sum_{j \in \mathbb{Z}} \widehat{\psi}_{j}-\sum_{j \approx k} \widehat{\psi}_{j}\right) \\
& =\left(1-\widehat{\phi}_{k}\right)\left(\sum_{j \in \mathbb{Z}} \widehat{\psi}_{j}-\widehat{\widetilde{\psi}}_{k}\right) .
\end{aligned}
$$

Here we use the fact $\left(1-\widehat{\phi}_{k}\right)\left(\sum_{j=-\infty}^{k-6} \widehat{\psi}_{j}\right)=0$ since they have disjoint supports.

That means

$$
\sum_{j=k+20}^{\infty} \Delta_{j} f=\left(1-S_{k}\right)\left(\sum_{j \in \mathbb{Z}} \Delta_{j}-\widetilde{\Delta}_{k}\right) f .
$$


Now we come back to the bi-parameter case. In the following arguments, we will omit the uniformly bounded constants $a_{j_{1}, j_{2}}$ and $b_{k_{1}, k_{2}}$ in (4.7)for simplicity, since one can see they do not play an essential role in our argument

$$
\begin{aligned}
& \sum_{j_{1}=k_{1}+20}^{\infty} \sum_{j_{2}=k_{2}+20}^{\infty} \Delta_{j_{1}} \Delta_{j_{2}} f \\
= & \left(1-S_{k_{1}}\right)\left(1-S_{k_{2}}\right)\left(\sum_{j_{1} \in \mathbb{Z}} \Delta_{j_{1}}-\widetilde{\Delta}_{k_{1}}\right)\left(\sum_{j_{2} \in \mathbb{Z}} \Delta_{j_{2}}-\widetilde{\Delta}_{k_{2}}\right) f \\
= & \left(1-S_{k_{1}}-S_{k_{2}}+S_{k_{1}} S_{k_{2}}\right)\left(\sum_{j_{1}, j_{2} \in \mathbb{Z}} \Delta_{j_{1}} \Delta_{j_{2}}-\widetilde{\Delta}_{k_{1}} \sum_{j_{2} \in \mathbb{Z}} \Delta_{j_{2}}-\sum_{j_{1} \in \mathbb{Z}} \Delta_{j_{1}} \widetilde{\Delta}_{k_{2}}+\widetilde{\Delta}_{k_{1}} \widetilde{\Delta}_{k_{2}}\right) f \\
:= & \left(1-S_{k_{1}}-S_{k_{2}}+S_{k_{1}} S_{k_{2}}\right)\left(O_{1}(f)+O_{2}(f)+O_{3}(f)+O_{4}(f)\right)
\end{aligned}
$$

We consider $\left(1-S_{k_{1}}-S_{k_{2}}+S_{k_{1}} S_{k_{2}}\right) O_{1}(f)$ first. Using $O_{1}(f)$, our operator becomes

$$
\begin{aligned}
I_{1}: & =\left\|\sum_{k_{1}, k_{2} \in \mathbb{Z}} \Delta_{k_{1}} S_{k_{2}} g S_{k_{1}} \Delta_{k_{2}} h O_{1}(f)\right\|_{L^{r}} \\
& \lesssim\left\|\sum_{k_{1}, k_{2} \in \mathbb{Z}} \Delta_{k_{1}} S_{k_{2}} g S_{k_{1}} \Delta_{k_{2}} h\right\|_{L^{s}}\left\|O_{1}(f)\right\|_{L^{p_{1}}} \\
& \lesssim\|g\|_{L^{p_{2}}}\|h\|_{L^{p_{3}}}\left\|O_{1}(f)\right\|_{L^{p_{1}}},
\end{aligned}
$$

where the first inequality follows from the Hölder's inequality with $1 / p_{2}+1 / p_{3}=1 / s$, and the second inequality follows from the classical bilinear bi-parameter multiplier boundedness.

Using $S_{k_{1}} S_{k_{2}} O_{1}(f)$, we have

$$
\begin{aligned}
I I_{1}: & =\left\|\sum_{k_{1}, k_{2} \in \mathbb{Z}} \Delta_{k_{1}} S_{k_{2}} g S_{k_{1}} \Delta_{k_{2}} h S_{k_{1}} S_{k_{2}} O_{1}(f)\right\|_{L^{r}} \\
& \lesssim\|g\|_{L^{p_{2}}}\|h\|_{L^{p_{3}}}\left\|O_{1}(f)\right\|_{L^{p_{1}}},
\end{aligned}
$$

where the inequality follows from the classical trilinear bi-parameter boundedness. Then our desired Hölder type estimate would hold since the classical linear theory gives

$$
\left\|O_{1}(f)\right\|_{L^{p_{1}}}=\left\|\sum_{j_{1}, j_{2} \in \mathbb{Z}} \Delta_{j_{1}} \Delta_{j_{2}} f\right\|_{L^{p_{1}}} \lesssim\|f\|_{L^{p_{1}}}, \quad 1<p_{1}<\infty .
$$


Note that actually $I_{1}$ corresponds to the boundedness of the following trilinear Fourier multiplier.

$$
\begin{aligned}
T_{I_{1}} & =\int \sum_{k_{1}, k_{2}} \widehat{\psi}_{k_{1}}\left(\eta_{1}\right) \widehat{\varphi}_{k_{2}}\left(\eta_{2}\right) \widehat{\varphi}_{k_{1}}\left(\zeta_{1}\right) \widehat{\psi}_{k_{2}}\left(\zeta_{2}\right) \widehat{O}_{1}(f)(\xi) \hat{g}(\eta) \hat{h}(\zeta) e^{2 \pi i x(\xi+\eta+\zeta)} d \xi d \eta d \zeta \\
& =\int\left(\sum_{k_{1}} \widehat{\psi}_{k_{1}}\left(\eta_{1}\right) \widehat{\varphi}_{k_{1}}\left(\zeta_{1}\right)\right)\left(\sum_{k_{2}} \widehat{\varphi}_{k_{2}}\left(\eta_{2}\right) \widehat{\psi}_{k_{2}}\left(\zeta_{2}\right)\right) \widehat{O}_{1}(f)(\xi) \hat{g}(\eta) \hat{h}(\zeta) e^{2 \pi i x(\xi+\eta+\zeta)} d \xi d \eta d \zeta \\
& =\int m^{\prime}\left(\eta_{1}, \zeta_{1}\right) m^{\prime \prime}\left(\eta_{2}, \zeta_{2}\right) \widehat{O}_{1}(f)(\xi) \hat{g}(\eta) \hat{h}(\zeta) e^{2 \pi i x(\xi+\eta+\zeta)} d \xi d \eta d \zeta \\
& =\int m^{\prime}\left(\eta_{1}, \zeta_{1}\right) m^{\prime \prime}\left(\eta_{2}, \zeta_{2}\right) \hat{g}(\eta) \hat{h}(\zeta) e^{2 \pi i x(\eta+\zeta)} d \eta d \zeta \cdot O_{1}(f)(x)
\end{aligned}
$$

This trilinear operator has a special bilinear symbol $m^{\prime}\left(\eta_{1}, \zeta_{1}\right) m^{\prime \prime}\left(\eta_{2}, \zeta_{2}\right)$, which is actually the product of $O_{1}(f)(x)$ and a bilinear bi-parameter multiplier. Then we can take advantage of the Hölder's inequality and a bilinear bi-parameter result. More precisely,

$$
\begin{aligned}
& \left\|T_{I_{1}}(f, g, h)\right\|_{L^{r}} \\
\lesssim & \left\|\int m^{\prime}\left(\eta_{1}, \zeta_{1}\right) m^{\prime \prime}\left(\eta_{2}, \zeta_{2}\right) \hat{g}(\eta) \hat{h}(\zeta) e^{2 \pi i x(\eta+\zeta)} d \eta d \zeta\right\|_{L^{s}}\left\|O_{1}(f)(x)\right\|_{L^{p_{1}}} \\
\lesssim & \|g\|_{L^{p_{2}}}\|h\|_{L^{p_{3}}}\|f\|_{L^{p_{1}}} .
\end{aligned}
$$

Similarly, the boundedness $I I_{1}$ corresponds to the trilinear Fourier multiplier

$$
\begin{aligned}
T_{I I_{1}}= & \int \sum_{k_{1}, k_{2}} \widehat{\varphi}_{k_{1}}\left(\xi_{1}\right) \widehat{\varphi}_{k_{2}}\left(\xi_{2}\right) \widehat{\psi}_{k_{1}}\left(\eta_{1}\right) \widehat{\varphi}_{k_{2}}\left(\eta_{2}\right) \widehat{\varphi}_{k_{1}}\left(\zeta_{1}\right) \widehat{\psi}_{k_{2}}\left(\zeta_{2}\right) \widehat{O}_{1}(f)(\xi) \hat{g}(\eta) \hat{h}(\zeta) e^{2 \pi i x(\xi+\eta+\zeta)} d \xi d \eta d \zeta \\
= & \int\left(\sum_{k_{1}} \widehat{\varphi}_{k_{1}}\left(\xi_{1}\right) \widehat{\psi}_{k_{1}}\left(\eta_{1}\right) \widehat{\varphi}_{k_{1}}\left(\zeta_{1}\right)\right)\left(\sum_{k_{2}} \widehat{\varphi}_{k_{2}}\left(\xi_{2}\right) \widehat{\varphi}_{k_{2}}\left(\eta_{2}\right) \widehat{\psi}_{k_{2}}\left(\zeta_{2}\right)\right) \\
& \widehat{O}_{1}(f)(\xi) \hat{g}(\eta) \hat{h}(\zeta) e^{2 \pi i x(\xi+\eta+\zeta)} d \xi d \eta d \zeta \\
= & \int m^{\prime}\left(\xi_{1}, \eta_{1}, \zeta_{1}\right) m^{\prime \prime}\left(\xi_{2}, \eta_{2}, \zeta_{2}\right) \widehat{O}_{1}(f)(\xi) \hat{g}(\eta) \hat{h}(\zeta) e^{2 \pi i x(\xi+\eta+\zeta)} d \xi d \eta d \zeta .
\end{aligned}
$$

Note that this is a standard bi-parameter trilinear Fourier multiplier, and that's why its Hölder type estimate holds.

Then we consider the terms like $S_{k_{1}} O(f)$ in (4.8)

$$
I I I_{1}:=\left\|\sum_{k_{1}, k_{2} \in \mathbb{Z}} \Delta_{k_{1}} S_{k_{2}} g S_{k_{1}} \Delta_{k_{2}} h S_{k_{1}} O_{1}(f)\right\|_{L^{r}}
$$


Similar as before, this corresponds to the following trilinear operator

$$
\begin{aligned}
T_{I I I_{1}}= & \int \sum_{k_{1}, k_{2}} \widehat{\varphi}_{k_{1}}\left(\xi_{1}\right) \widehat{\psi}_{k_{1}}\left(\eta_{1}\right) \widehat{\varphi}_{k_{2}}\left(\eta_{2}\right) \widehat{\varphi}_{k_{1}}\left(\zeta_{1}\right) \widehat{\psi}_{k_{2}}\left(\zeta_{2}\right) \widehat{O}_{1}(f)(\xi) \hat{g}(\eta) \hat{h}(\zeta) e^{2 \pi i x(\xi+\eta+\zeta)} d \xi d \eta d \zeta \\
= & \int\left(\sum_{k_{1}} \widehat{\varphi}_{k_{1}}\left(\xi_{1}\right) \widehat{\psi}_{k_{1}}\left(\eta_{1}\right) \widehat{\varphi}_{k_{1}}\left(\zeta_{1}\right)\right)\left(\sum_{k_{2}} \widehat{\varphi}_{k_{2}}\left(\eta_{2}\right) \widehat{\psi}_{k_{2}}\left(\zeta_{2}\right)\right) \\
& \widehat{O}_{1}(f)(\xi) \hat{g}(\eta) \hat{h}(\zeta) e^{2 \pi i x(\xi+\eta+\zeta)} d \xi d \eta d \zeta \\
= & \int m^{\prime}\left(\xi_{1}, \eta_{1}, \zeta_{1}\right) m^{\prime \prime}\left(\eta_{2}, \zeta_{2}\right) \widehat{O}_{1}(f)(\xi) \hat{g}(\eta) \hat{h}(\zeta) e^{2 \pi i x(\xi+\eta+\zeta)} d \xi d \eta d \zeta .
\end{aligned}
$$

Remark 1. Note that this trilinear multiplier has a symbol

$$
m(\xi, \eta, \zeta)=m_{1}\left(\xi_{1}, \eta_{1}, \zeta_{1}\right) m_{2}\left(\eta_{2}, \zeta_{2}\right)
$$

where one variable is missing for the second parameter, i.e., the function $f$ is not actually transformed in the second variable. Such a symbol can be interpreted as an intermediate case between the previous $I_{1}$ and $I I_{1}$, and that's why we conjecture that Hölder type estimates should hold for operator. If we make the assumption that this operator is bounded, we have the following.

$$
\left\|T_{I I I_{1}}(f, g, h)\right\|_{L^{r}} \lesssim\|g\|_{L^{p_{2}}}\|h\|_{L^{p_{3}}}\left\|O_{1}(f)\right\|_{L^{p_{1}}} \lesssim\|g\|_{L^{p_{2}}}\|h\|_{L^{p_{3}}}\|f\|_{L^{p_{1}}}
$$

Now we consider $\left(1-S_{k_{1}}-S_{k_{2}}+S_{k_{1}} S_{k_{2}}\right) O_{4}(f)$. We still consider the three parts $O_{4}(f), S_{k_{1}} S_{k_{2}} O_{4}(f)$ and $S_{k_{1}} O_{4}(f)$. Recall

$$
O_{4}(f)=\left(\widetilde{\Delta}_{k_{1}} \widetilde{\Delta}_{k_{2}}\right) f
$$

Thus, with $O_{4}(f)$, we have

$$
\begin{aligned}
I_{4}: & =\left\|\sum_{k_{1}, k_{2} \in \mathbb{Z}} \Delta_{k_{1}} S_{k_{2}} g S_{k_{1}} \Delta_{k_{2}} h O_{4}(f)\right\|_{L^{r}} \\
& =\left\|\sum_{k_{1}, k_{2} \in \mathbb{Z}} \Delta_{k_{1}} S_{k_{2}} g S_{k_{1}} \Delta_{k_{2}} h \widetilde{\Delta}_{k_{1}} \widetilde{\Delta}_{k_{2}} f\right\|_{L^{r}} \\
& \lesssim\|g\|_{L^{p_{2}}}\|h\|_{L^{p_{3}}}\|f\|_{L^{p_{1}}},
\end{aligned}
$$

where the estimate follows from the classical trilinear bi-parameter multiplier boundedness, as what we argued for $T_{I_{1}}$.

With $S_{k_{1}} S_{k_{2}} O_{4}(f)$

$$
I I_{4}:=\left\|\sum_{k_{1}, k_{2} \in \mathbb{Z}} \Delta_{k_{1}} S_{k_{2}} g S_{k_{1}} \Delta_{k_{2}} h S_{k_{1}} S_{k_{2}} O_{4}(f)\right\|_{L^{r}}
$$




$$
=\left\|\sum_{k_{1}, k_{2} \in \mathbb{Z}} \Delta_{k_{1}} S_{k_{2}} g S_{k_{1}} \Delta_{k_{2}} h S_{k_{1}} S_{k_{2}} \widetilde{\Delta}_{k_{1}} \widetilde{\Delta}_{k_{2}} f\right\|_{L^{r}}
$$

Recall that

Thus, we can write

$$
\operatorname{supp} \widehat{\phi}_{k} \subset\left\{|\xi| \leq 2^{k-2}\right\}
$$

$$
\begin{aligned}
& \operatorname{supp} \underset{\widehat{\psi}_{k}}{ } \subset\left\{2^{k-1} \leq|\xi| \leq 2^{k+1}\right\} \\
& \operatorname{supp} \underset{\widetilde{\psi}_{k}}{ } \subset\left\{2^{k-6} \leq|\xi| \leq 2^{k+20}\right\}
\end{aligned}
$$

$$
S_{k_{1}} S_{k_{2}} \widetilde{\Delta}_{k_{1}} \widetilde{\Delta}_{k_{2}} f=\widetilde{\Delta}_{k_{1}}^{\prime} \widetilde{\Delta}_{k_{2}}^{\prime} f
$$

where $\widetilde{\Delta}_{k}^{\prime} f=\left(\widehat{\widetilde{\psi}_{k}^{\prime}} \hat{f}\right)^{\vee}=\left(\widehat{\phi}_{k} \widehat{\widetilde{\psi}_{k}} \hat{f}\right)^{\vee}$. Note that $\operatorname{supp} \widehat{\widetilde{\psi}_{k}^{\prime}} \subset\left\{2^{k-6} \leq|\xi| \leq 2^{k-2}\right\}$.

Then the estimate

$$
\begin{aligned}
I I_{4}: & =\left\|\sum_{k_{1}, k_{2} \in \mathbb{Z}} \Delta_{k_{1}} S_{k_{2}} g S_{k_{1}} \Delta_{k_{2}} h S_{k_{1}} S_{k_{2}} O_{4}(f)\right\|_{L^{r}} \\
& =\left\|\sum_{k_{1}, k_{2} \in \mathbb{Z}} \Delta_{k_{1}} S_{k_{2}} g S_{k_{1}} \Delta_{k_{2}} h \widetilde{\Delta}_{k_{1}}^{\prime} \widetilde{\Delta}_{k_{2}}^{\prime} f\right\|_{L^{r}} \\
& \lesssim\|g\|_{L^{p_{2}}}\|h\|_{L^{p_{3}}}\|f\|_{L^{p_{1}}}
\end{aligned}
$$

follows from the classical trilinear bi-parameter boundedness.

With $S_{k_{1}} O_{4}(f)$, we have

$$
\begin{aligned}
I I I_{4}: & =\left\|\sum_{k_{1}, k_{2} \in \mathbb{Z}} \Delta_{k_{1}} S_{k_{2}} g S_{k_{1}} \Delta_{k_{2}} h S_{k_{1}} O_{4}(f)\right\|_{L^{r}} \\
& =\left\|\sum_{k_{1}, k_{2} \in \mathbb{Z}} \Delta_{k_{1}} S_{k_{2}} g S_{k_{1}} \Delta_{k_{2}} h S_{k_{1}} \widetilde{\Delta}_{k_{1}} \widetilde{\Delta}_{k_{2}} f\right\|_{L^{r}} \\
& =\left\|\sum_{k_{1}, k_{2} \in \mathbb{Z}} \Delta_{k_{1}} S_{k_{2}} g S_{k_{1}} \Delta_{k_{2}} h \widetilde{\Delta}_{k_{1}}^{\prime} \widetilde{\Delta}_{k_{2}} f\right\|_{L^{r}} \\
& \lesssim\|g\|_{L^{p_{2}}}\|h\|_{L^{p_{3}}}\|f\|_{L^{p_{1}}}
\end{aligned}
$$

where we use the same trick

$$
S_{k_{1}} \widetilde{\Delta}_{k_{1}} \widetilde{\Delta}_{k_{2}} f=\widetilde{\Delta}_{k_{1}}^{\prime} \widetilde{\Delta}_{k_{2}} f
$$


Then we consider the last situation left in (4.8), which is $O_{2}(f)$. Using the same argument as above, we can write

$$
\begin{aligned}
& \left(1-S_{k_{1}}-S_{k_{2}}+S_{k_{1}} S_{k_{2}}\right) O_{2}(f) \\
= & \left(1-S_{k_{1}}-S_{k_{2}}+S_{k_{1}} S_{k_{2}}\right) \widetilde{\Delta}_{k_{1}} \sum_{j_{2} \in \mathbb{Z}} \Delta_{j_{2}}(f) \\
= & \widetilde{\Delta}_{k_{1}}\left(\sum_{j_{2} \in \mathbb{Z}} \Delta_{j_{2}} f\right)-\widetilde{\Delta}_{k_{1}}^{\prime}\left(\sum_{j_{2} \in \mathbb{Z}} \Delta_{j_{2}} f\right)-\widetilde{\Delta}_{k_{1}} S_{k_{2}}\left(\sum_{j_{2} \in \mathbb{Z}} \Delta_{j_{2}} f\right)+\widetilde{\Delta}_{k_{1}}^{\prime} S_{k_{2}}\left(\sum_{j_{2} \in \mathbb{Z}} \Delta_{j_{2}} f\right) \\
:= & \widetilde{\Delta}_{k_{1}} Q(f)-\widetilde{\Delta}_{k_{1}}^{\prime} Q(f)-\widetilde{\Delta}_{k_{1}} S_{k_{2}} Q(f)+\widetilde{\Delta}_{k_{1}}^{\prime} S_{k_{2}} Q(f),
\end{aligned}
$$

where obviously

$$
\|Q(f)\|_{L^{p_{1}}}=\left\|\sum_{j_{2} \in \mathbb{Z}} \Delta_{j_{2}} f\right\|_{L^{p_{1}}} \lesssim\|f\|_{L^{p_{1}}}
$$

Now we consider the $L^{r}$ norm of .

$$
\begin{aligned}
& \left\|\sum_{k_{1}, k_{2} \in \mathbb{Z}} \Delta_{k_{1}} S_{k_{2}} g S_{k_{1}} \Delta_{k_{2}} h\left(1-S_{k_{1}}-S_{k_{2}}+S_{k_{1}} S_{k_{2}}\right) O_{2}(f)\right\|_{L^{r}} \\
= & \left\|\sum_{k_{1}, k_{2} \in \mathbb{Z}} \Delta_{k_{1}} S_{k_{2}} g S_{k_{1}} \Delta_{k_{2}} h\left(\widetilde{\Delta}_{k_{1}} Q(f)-\widetilde{\Delta}_{k_{1}}^{\prime} Q(f)-\widetilde{\Delta}_{k_{1}} S_{k_{2}} Q(f)+\widetilde{\Delta}_{k_{1}}^{\prime} S_{k_{2}} Q(f)\right)\right\|_{L^{r}} \\
\lesssim & \left\|\sum_{k_{1}, k_{2} \in \mathbb{Z}} \Delta_{k_{1}} S_{k_{2}} g S_{k_{1}} \Delta_{k_{2}} h \widetilde{\Delta}_{k_{1}} Q(f)\right\|_{L^{r}}+\left\|\sum_{k_{1}, k_{2} \in \mathbb{Z}} \Delta_{k_{1}} S_{k_{2}} g S_{k_{1}} \Delta_{k_{2}} h \widetilde{\Delta}_{k_{1}}^{\prime} Q(f)\right\|_{L^{r}} \\
+ & \left\|\sum_{k_{1}, k_{2} \in \mathbb{Z}} \Delta_{k_{1}} S_{k_{2}} g S_{k_{1}} \Delta_{k_{2}} h \widetilde{\Delta}_{k_{1}} S_{k_{2}} Q(f)\right\|_{L^{r}}+\left\|\sum_{k_{1}, k_{2} \in \mathbb{Z}} \Delta_{k_{1}} S_{k_{2}} g S_{k_{1}} \Delta_{k_{2}} h \widetilde{\Delta}_{k_{1}}^{\prime} S_{k_{2}} Q(f)\right\|_{L^{r}} \\
\lesssim & \|g\|_{L^{p_{2}}}\|h\|_{L^{p_{3}}}\|Q(f)\|_{L^{p_{1}}} \lesssim\|g\|_{L^{p_{2}}}\|h\|_{L^{p_{3}}}\|f\|_{L^{p_{1}}}
\end{aligned}
$$

where the estimates for the last two terms in (4.9) are given by the classical trilinear bi-parameter boundedness, while the first two terms correspond to situation $I I I_{1}$ in Remark 1, where in the second parameter we just have a bilinear multiplier.

Moreover, this approach should work for all of the operators $T^{1}, T^{2}, T^{3}$ and $T^{4}$, since more $\psi$-type functions appear in $T^{1}, T^{2}$ and $T^{3}$. In fact, these four operators differ from each other in the decomposition for $g$ and $h$ part. However, what they have in common is that for each parameter, there is at least one $\psi$-function or $\Delta$, and this is the key in our argument. Thus, the boundedness of (1.7) would follow, as long as the assumption in Remark 1 is true. 


\section{Reduction of Theorem 1.10}

In this section we give the idea to prove Theorem 1.10, the strategy is to reduce the pseudo-differential operator to a localized version. From now on we will redefine the functions that were used in the previous sections: $\psi, \varphi$, and $\phi$.

First pick two sequences of smooth functions $\left(\varphi_{n}\right)_{n \in \mathbb{Z}},\left(\varphi_{m}^{\prime}\right)_{m \in \mathbb{Z}}$ such that $\operatorname{supp} \varphi_{n} \subseteq$ $[n-1, n+1]$ and $\operatorname{supp} \varphi_{m}^{\prime} \subseteq[m-1, m+1]$ satisfying

$$
\sum_{n \in \mathbb{Z}} \varphi_{n}\left(x_{1}\right)=1, \quad \sum_{m \in \mathbb{Z}} \varphi_{m}^{\prime}\left(x_{2}\right)=1, \quad \text { where }\left(x_{1}, x_{2}\right) \in \mathbb{R}^{2} .
$$

Then we can decompose the operator $T_{a b}$ in (1.9) as

$$
T_{a b}=\sum_{n, m \in \mathbb{Z}} T_{a b}^{n, m}
$$

where

$$
T_{a b}^{n, m}(f, g, h)(x):=T_{a b}(f, g, h)(x) \varphi_{n}\left(x_{1}\right) \varphi_{m}^{\prime}\left(x_{2}\right) .
$$

Suppose we can prove the estimate

$$
\left\|T_{a b}^{n, m}(f, g, h)\right\|_{r} \lesssim\left\|f \tilde{\chi}_{R_{n m}}\right\|_{p_{1}}\left\|g \tilde{\chi}_{R_{n m}}\right\|_{p_{2}}\left\|h \tilde{\chi}_{R_{n m}}\right\|_{p_{3}},
$$

where $R_{n m}=I_{n} \times J_{m}, I_{n}=[n, n+1], J_{m}=[m, m+1]$ and $\tilde{\chi}_{R_{n m}}=\tilde{\chi}_{I_{n}}\left(x_{1}\right) \times \tilde{\chi}_{J_{m}}\left(x_{2}\right)$ as defined in (2.1).

Then our main Theorem 1.10 can be proved by the following estimate

$$
\begin{aligned}
\left\|T_{a b}(f, g, h)\right\|_{r} & \lesssim\left(\sum_{n, m \in \mathbb{Z}}\left\|T_{a b}^{n, m}(f, g, h)\right\|_{r}^{r}\right)^{1 / r} \\
& \lesssim\left(\sum_{n, m \in \mathbb{Z}}\left\|f \tilde{\chi}_{R_{n m}}\right\|_{p_{1}}^{r}\left\|g \tilde{\chi}_{R_{n m}}\right\|_{p_{2}}^{r}\left\|h \tilde{\chi}_{R_{n m}}\right\|_{p_{3}}^{r}\right)^{1 / r} \\
& \lesssim\left(\sum_{n, m \in \mathbb{Z}}\left\|f \tilde{\chi}_{R_{n m}}\right\|_{p_{1}}^{p_{1}}\right)^{1 / p_{1}}\left(\sum_{n, m \in \mathbb{Z}}\left\|g \tilde{\chi}_{R_{n, m}}\right\|_{p_{2}}^{p_{2}}\right)^{1 / p_{2}}\left(\sum_{n, m \in \mathbb{Z}}\left\|h \tilde{\chi}_{R_{n m}}\right\|_{p_{3}}^{p_{3}}\right)^{1 / p_{3}} \\
& \lesssim\|f\|_{p_{1}}\|g\|_{p_{2}}\|h\|_{p_{3}} .
\end{aligned}
$$

Thus, we only need to prove (5.1).

Consider that for a fixed $n_{0}, m_{0} \in \mathbb{Z}$, we have

$$
\begin{aligned}
T_{a b}^{n_{0}, m_{0}}(f, g, h)(x)= & \int_{\mathbb{R}^{6}} a(x, \xi, \eta, \zeta) \tilde{\varphi}_{n_{0}}\left(x_{1}\right) \tilde{\varphi}_{m_{0}}^{\prime}\left(x_{2}\right) b(x, \eta, \zeta) \tilde{\varphi}_{n_{0}}\left(x_{1}\right) \tilde{\varphi}_{m_{0}}^{\prime}\left(x_{2}\right) \\
& \cdot \varphi_{n_{0}}\left(x_{1}\right) \varphi_{m_{0}}^{\prime}\left(x_{2}\right) \hat{f}(\xi) \hat{g}(\eta) \hat{h}(\zeta) e^{2 \pi i x(\xi+\eta+\zeta)} d \xi d \eta d \zeta,
\end{aligned}
$$

where $\tilde{\varphi}_{n_{0}}, \tilde{\varphi}_{m_{0}}^{\prime}$ are smooth functions supported on the intervals $\left[n_{0}-2, n_{0}+2\right]$, $\left[m_{0}-2, m_{0}+2\right]$, which equal 1 on the supports of $\varphi_{n_{0}}, \varphi_{m_{0}}^{\prime}$ respectively. Then we rewrite the symbols $a(x, \xi, \eta, \zeta) \tilde{\varphi}_{n_{0}}\left(x_{1}\right) \tilde{\varphi}_{m_{0}}^{\prime}\left(x_{2}\right)$ and $b(x, \eta, \zeta) \tilde{\varphi}_{n_{0}}\left(x_{1}\right) \tilde{\varphi}_{m_{0}}^{\prime}\left(x_{2}\right)$ by using Fourier series with respect to the $x$ variable 


$$
\begin{gathered}
a(x, \xi, \eta, \zeta) \tilde{\varphi}_{n_{0}}\left(x_{1}\right) \tilde{\varphi}_{m_{0}}^{\prime}\left(x_{2}\right)=\sum_{l_{1}, l_{2} \in \mathbb{Z}} a_{l_{1}, l_{2}}(\xi, \eta, \zeta, \zeta) e^{2 \pi i\left(x_{1} l_{1}+x_{2} l_{2}\right)} \\
b(x, \eta, \zeta) \tilde{\varphi}_{n_{0}}\left(x_{1}\right) \tilde{\varphi}_{m_{0}}^{\prime}\left(x_{2}\right)=\sum_{l_{1}^{\prime}, l_{2}^{\prime} \in \mathbb{Z}} b_{l_{1}^{\prime}, l_{2}^{\prime}}(\eta, \zeta) e^{2 \pi i\left(x_{1} l_{1}^{\prime}+x_{2} l_{2}^{\prime}\right)}
\end{gathered}
$$

where

$$
\begin{aligned}
a_{l_{1}, l_{2}}(\xi, \eta, \zeta) & =\int_{\mathbb{R}^{2}} a(x, \xi, \eta, \zeta) \tilde{\varphi}_{n_{0}}\left(x_{1}\right) \tilde{\varphi}_{m_{0}}^{\prime}\left(x_{2}\right) e^{-2 \pi i\left(x_{1} l_{1}+x_{2} l_{2}\right)} d x \\
b_{l_{1}^{\prime}, l_{2}^{\prime}}(\eta, \zeta) & =\int_{\mathbb{R}^{2}} b(x, \eta, \zeta) \tilde{\varphi}_{n_{0}}\left(x_{1}\right) \tilde{\varphi}_{m_{0}}^{\prime}\left(x_{2}\right) e^{-2 \pi i\left(x_{1} l_{1}^{\prime}+x_{2} l_{2}^{\prime}\right)} d x
\end{aligned}
$$

By taking advantage of conditions (1.10) we have

$$
\begin{gathered}
\lesssim \frac{1}{\left(1+\left|\left(l_{1}, l_{2}\right)\right|\right)^{M}} \frac{\left|\partial_{\xi_{1}, \eta_{1}, \zeta_{1}}^{\alpha_{1}, \beta_{1}, \gamma_{1}} \partial_{\xi_{2}, \eta_{2}, \zeta_{2}}^{\alpha_{2}, \beta_{2}, \gamma_{2}} a_{l_{1}, l_{2}}(\xi, \eta, \zeta)\right|}{\left(1+\left|\xi_{1}\right|+\left|\eta_{1}\right|+\left|\zeta_{1}\right|\right)^{\alpha_{1}+\beta_{1}+\gamma_{1}}} \frac{1}{\left(1+\left|\xi_{2}\right|+\left|\eta_{2}\right|+\left|\zeta_{2}\right|\right)^{\alpha_{2}+\beta_{2}+\gamma_{2}}} \\
\left|\partial_{\eta_{1}, \zeta_{1}}^{\beta_{1}, \gamma_{1}} \partial_{\eta_{2}, \zeta_{2}}^{\beta_{2}, \gamma_{2}} b_{l_{1}^{\prime}, l_{2}^{\prime}}(\eta, \zeta)\right| \lesssim \frac{1}{\left(1+\left|\left(l_{1}^{\prime}, l_{2}^{\prime}\right)\right|\right)^{M}} \frac{1}{\left(1+\left|\eta_{1}\right|+\left|\zeta_{1}\right|\right)^{\beta_{1}+\gamma_{1}}} \frac{1}{\left(1+\left|\eta_{2}\right|+\left|\zeta_{2}\right|\right)^{\beta_{2}+\gamma_{2}}}
\end{gathered}
$$

for a large number $M$ and all indices $\alpha_{1}, \alpha_{2}, \beta_{1}, \beta_{2}, \gamma_{1}, \gamma_{2}$. Note that the decay in $l_{1}, l_{2}, l_{1}^{\prime}, l_{2}^{\prime}$ allows one to take summation $T_{a b}^{n_{0}, m_{0}}=\sum_{l_{1}, l_{2}, l_{1}^{\prime}, l_{2}^{\prime}} T_{a b}^{n_{0}, m_{0}, l_{1}, l_{2}, l_{1}^{\prime}, l_{2}^{\prime}}$, where

$$
\begin{gathered}
T_{a b}^{n_{0}, m_{0}, l_{1}, l_{2}, l_{1}^{\prime}, l_{2}^{\prime}}(f, g, h)(x)= \\
\left(\int_{\mathbb{R}^{6}} a_{l_{1}, l_{2}}(\xi, \eta, \zeta) b_{l_{1}^{\prime}, l_{2}^{\prime}}(\eta, \zeta) \hat{f}(\xi) \hat{g}(\eta) \hat{h}(\zeta) e^{2 \pi i x(\xi+\eta+\zeta)} d \xi d \eta d \zeta\right) \varphi_{n_{0}}\left(x_{1}\right) \varphi_{m_{0}}^{\prime}\left(x_{2}\right) .
\end{gathered}
$$

That means we only need to consider the case for $l_{1}, l_{2}, l_{1}^{\prime}, l_{2}^{\prime}=0$. For simplicity, we denote it by

$$
\begin{gathered}
T_{a b}^{n_{0}, m_{0}, 0,0}(f, g, h)(x)= \\
\left(\int_{\mathbb{R}^{6}} a_{0}(\xi, \eta, \zeta) b_{0}(\eta, \zeta) \hat{f}(\xi) \hat{g}(\eta) \hat{h}(\zeta) e^{2 \pi i x(\xi+\eta+\zeta)} d \xi d \eta d \zeta\right) \varphi_{n_{0}}\left(x_{1}\right) \varphi_{m_{0}}^{\prime}\left(x_{2}\right),
\end{gathered}
$$

where the symbols $a_{0}, b_{0}$ satisfy the following conditions

$$
\begin{gathered}
\lesssim \frac{1}{\partial_{\xi_{1}, \eta_{1}, \zeta_{1}}^{\alpha_{1}, \beta_{1}, \gamma_{1}} \partial_{\xi_{2}, \eta_{2}, \zeta_{2}}^{\alpha_{2}, \beta_{2}, \gamma_{2}} a_{0}(\xi, \eta, \zeta) \mid} \\
\left|\partial_{\eta_{1}, \zeta_{1}}^{\beta_{1}, \gamma_{1}} \partial_{\eta_{2}, \zeta_{2}}^{\beta_{2}, \gamma_{2}} b_{0}(\eta, \zeta)\right| \lesssim \frac{1}{\left(1+\left|\eta_{1}\right|+\left|\zeta_{1}\right|\right)^{\beta_{1}+\gamma_{1}}} \frac{1}{\left(1+\left|\eta_{2}\right|+\left|\zeta_{2}\right|\right)^{\beta_{2}+\gamma_{2}}}
\end{gathered}
$$

for all indices $\alpha_{1}, \alpha_{2}, \beta_{1}, \beta_{2}, \gamma_{1}, \gamma_{2}$. 
By translation invariance, we only need to prove the following localized result for $n_{0}, m_{0}=0$.

Theorem 5.1. For $1<p_{1}, p_{2}, p_{3}<\infty$, and $1 / p_{1}+1 / p_{2}+1 / p_{3}=1 / r$ the operator

$$
\begin{gathered}
T_{a b}^{0}:=T_{a b}^{0,0,0,0}(f, g, h)(x)= \\
\left(\int_{\mathbb{R}^{6}} a_{0}(\xi, \eta, \zeta) b_{0}(\eta, \zeta) \hat{f}(\xi) \hat{g}(\eta) \hat{h}(\zeta) e^{2 \pi i x(\xi+\eta+\zeta)} d \xi d \eta d \zeta\right) \varphi_{0}\left(x_{1}\right) \varphi_{0}^{\prime}\left(x_{2}\right)
\end{gathered}
$$

has the following boundedness property

$$
\left\|T_{a b}^{0}(f, g, h)\right\|_{r} \lesssim\left\|f \tilde{\chi}_{R_{00}}\right\|_{p_{1}}\left\|g \tilde{\chi}_{R_{00}}\right\|_{p_{2}}\left\|h \tilde{\chi}_{R_{00}}\right\|_{p_{3}},
$$

where $\varphi_{0}, \varphi_{0}^{\prime}$ are smooth functions supported within $I^{0}=[-1,1], \tilde{\chi}_{R_{00}}(x)=\tilde{\chi}_{I^{0}}\left(x_{1}\right)$. $\tilde{\chi}_{I^{0}}\left(x_{2}\right)$ and $a_{0}, b_{0}$ satisfy the conditions (5.2).

In short, the proof of Theorem 1.10 can be reduced to the above theorem, and in the next section we will show how to deal with the operator in (5.3).

\section{Proof of Theorem 5.1}

In this section we prove Theorem 5.1. The first step is to use Fourier series as before and rewrite the operator (5.3). Here we make use of the fact that the conditions (5.2) do not involve any singularity. That means that there is no problem when the variables $\xi, \eta, \zeta$ are close to zero. More precisely, we can modify the Littlewood-Paley decomposition as follows.

Let $\varphi$ be a Schwartz function such that $\operatorname{supp} \hat{\varphi} \subseteq[-1,1]$ and $\hat{\varphi}(u)=1$ on $[-1 / 2,1 / 2]$, and let $\psi$ be the Schwartz function satisfying

$$
\hat{\psi}(u):=\hat{\varphi}(u / 2)-\hat{\varphi}(u),
$$

and let

Note that

$$
\widehat{\psi_{k}}(\cdot)=\widehat{\psi}\left(\cdot / 2^{k}\right) \quad \text { and } \quad \widehat{\psi_{-1}}(\cdot)=\hat{\varphi}(\cdot)
$$

$$
1=\sum_{k \geq-1} \widehat{\psi_{k}}, \quad \text { where supp } \hat{\psi} \subseteq\left[-2^{k+1},-2^{k-1}\right] \cup\left[2^{k-1}, 2^{k+1}\right] \text { for } k \geq 0 .
$$

The key thing here is that one does not have to decompose the identity near 0 . Moreover, for any smooth function $\phi$ supported on a closed interval, we write $\tilde{\phi}$ to denote a smooth function that is supported on a slightly larger interval and equal to 1 on the support of $\phi$. Actually, we will use $\phi$ to represent either a $\varphi$ function or a $\psi$ function. For simplicity, let us consider the single-parameter case first, i.e. temporarily assume $\xi, \eta, \zeta \in \mathbb{R}$. By expanding in Fourier series as before, it can be seen to be sufficient to replace the symbols $a_{0}(\xi, \eta, \zeta)$ and $b_{0}(\xi, \eta, \zeta)$ with

$$
a_{0}(\xi, \eta, \zeta)=\sum_{k \geq 0} \widehat{\phi_{k}^{1}}(\xi) \widehat{\phi_{k}^{2}}(\eta) \widehat{\phi_{k}^{3}}(\zeta)+\hat{\varphi}(\xi) \hat{\varphi}(\eta) \hat{\varphi}(\zeta)
$$


where at least one of the families $\left.\left(\widehat{\phi_{k}^{1}}(\xi)\right)_{k}, \widehat{\left(\phi_{k}^{2}\right.}(\eta)\right)_{k}$, and $\left.\widehat{\left(\phi_{k}^{3}\right.}(\zeta)\right)_{k}$ is supported away from the origin. Similarly,

$$
b_{0}(\eta, \zeta)=\sum_{k \geq 0} \widehat{\phi_{k}^{2}}(\eta) \widehat{\phi_{k}^{3}}(\zeta)+\hat{\varphi}(\eta) \hat{\varphi}(\zeta)
$$

where at least one of the families $\left.\left(\widehat{\phi_{k}^{2}}(\eta)\right)_{k}, \widehat{\left(\phi_{k}^{3}\right.}(\zeta)\right)_{k}$ is supported away from the origin. Now we can replace the symbol $a_{0}(\xi, \eta, \zeta) b_{0}(\eta, \zeta)$ by

$$
\begin{aligned}
& a_{0}(\xi, \eta, \zeta) b_{0}(\eta, \zeta) \\
= & \left(\sum_{k_{1} \geq 0} \widehat{\phi_{k_{1}}^{1}}(\xi) \widehat{\phi_{k_{1}}^{2}}(\eta) \widehat{\phi_{k_{1}}^{3}}(\zeta)+\hat{\varphi}(\xi) \hat{\varphi}(\eta) \hat{\varphi}(\zeta)\right)\left(\sum_{k_{2} \geq 0} \widehat{\phi_{k_{2}}^{2}}(\eta) \widehat{\phi_{k_{2}}^{3}}(\zeta)+\hat{\varphi}(\eta) \hat{\varphi}(\zeta)\right) \\
\approx & \left(\sum_{k_{1} \geq 0} \widehat{\phi_{k_{1}}^{1}}(\xi) \widehat{\phi_{k_{1}}^{2}}(\eta) \widehat{\phi_{k_{1}}^{3}}(\zeta) \sum_{k_{2} \geq 0} \widehat{\phi_{k_{2}}^{1}}(\eta) \widehat{\phi_{k_{2}}^{2}}(\zeta)\right)+\left(\sum_{k_{1} \geq 0} \widehat{\phi_{k_{1}}^{1}}(\xi) \widehat{\phi_{k_{1}}^{2}}(\eta) \widehat{\phi_{k_{1}}^{3}}(\zeta)\right) \hat{\varphi}(\eta) \hat{\varphi}(\zeta) \\
& +\left(\sum_{k_{2} \geq 0} \widehat{\phi_{k_{2}}^{1}}(\eta) \widehat{\phi_{k_{2}}^{2}}(\zeta)\right) \hat{\varphi}(\xi) \hat{\varphi}(\eta) \varphi(\zeta)+\hat{\varphi}(\xi) \hat{\varphi}(\eta) \hat{\varphi}(\zeta) \hat{\varphi}(\eta) \hat{\varphi}(\zeta) \\
= & \left(\sum_{k_{1} \geq 0} \widehat{\phi_{k_{1}}^{1}}(\xi) \widehat{\phi_{k_{1}}^{2}}(\eta) \widehat{\phi_{k_{1}}^{3}}(\zeta) \sum_{k_{2} \ll k_{1}} \widehat{\phi_{k_{2}}^{1}}(\eta) \widehat{\phi_{k_{2}}^{2}}(\zeta)\right) \\
& +\left(\sum_{k_{1} \geq 0} \widehat{\phi_{k_{1}}^{1}}(\xi) \widehat{\phi_{k_{1}}^{2}}(\eta) \widehat{\phi_{k_{1}}^{3}}(\zeta) \sum_{k_{2} \gg k_{1}} \widehat{\phi_{k_{2}}^{1}}(\eta) \widehat{\phi_{k_{2}}^{2}}(\zeta)\right) \\
& +\left(\sum_{k_{1} \geq 0} \widehat{\phi_{k_{1}}^{1}}(\xi) \widehat{\phi_{k_{1}}^{2}}(\eta) \widehat{\phi_{k_{1}}^{3}}(\zeta) \sum_{k_{2} \simeq k_{1}} \widehat{\phi_{k_{2}}^{1}}(\eta) \widehat{\phi_{k_{2}}^{2}}(\zeta)\right) \\
& +\left(\sum_{k_{1} \geq 0} \widehat{\phi_{k_{1}}^{1}}(\xi) \widehat{\phi_{k_{1}}^{2}}(\eta) \widehat{\phi_{k_{1}}^{3}}(\zeta)\right) \hat{\varphi}(\eta) \hat{\varphi}(\zeta) \\
& +\left(\sum_{k_{2} \geq 0} \widehat{\phi_{k_{2}}^{1}}(\eta) \widehat{\phi_{k_{2}}^{2}}(\zeta)\right) \hat{\varphi}(\xi) \hat{\varphi}(\eta) \hat{\varphi}(\zeta)+\hat{\varphi}(\xi) \hat{\varphi}(\eta) \hat{\varphi}(\eta) \varphi(\zeta) \hat{\varphi}(\zeta) \\
:= & (E+F+G+1)
\end{aligned}
$$

First note that it is not possible that $k_{2} \gg k_{1}$, which implies that $F=0$, since at least one of $\left.\left(\widehat{\phi_{k}^{2}}(\eta)\right)_{k}, \widehat{\left(\phi_{k}^{3}\right.}(\zeta)\right)_{k}$ is supported away from the origin.

To take care of other terms, the essential idea here is to compare the sizes of the supports of $\xi, \eta, \zeta$, as we have done before. Roughly speaking, one can consider the following two cases:

- Case I: When $\{|\xi| \leq c(|\eta|+|\zeta|)\}$ for some constant $c$, i.e. the terms $G, K, L$, such terms correspond to the following estimate of the symbol

$$
\left|\partial_{\xi}^{\alpha} \partial_{\eta}^{\beta} \partial_{\zeta}^{\gamma} a_{0}(\xi, \eta, \zeta) b_{0}(\eta, \zeta)\right| \lesssim \frac{1}{(|\xi|+|\eta|+|\gamma|)^{\alpha+\beta+\gamma}}
$$

- Case II: When $\left\{|\xi| \geq \frac{1}{2} c(|\eta|+|\zeta|)\right\}$, i.e. the terms $E, G$ correspond to the operators in Definition [5 - see [18, 19] for more details. 
With the above argument, we can simplify (6.1) to

$$
E+F+G+H+K+L \approx E+G,
$$

where we use $E$ to represent Case II, and $G$ to represent Case I.

Now we come back to the bi-parameter case. By doing the decomposition as above in each parameter, i.e. $\left(\xi_{1}, \eta_{1}, \zeta_{1}\right)$ and $\left(\xi_{2}, \eta_{2}, \zeta_{2}\right)$, one should have four cases to estimate. More precisely, we can replace $a_{0}(\xi, \eta, \zeta) b_{0}(\eta, \zeta)$ by

$$
a_{0}(\xi, \eta, \zeta) b_{0}(\eta, \zeta) \approx(E+G)\left(\xi_{1}, \eta_{1}, \zeta_{1}\right)\left(E^{\prime}+G^{\prime}\right)\left(\xi_{2}, \eta_{2}, \zeta_{2}\right)
$$

Correspondingly, the localized operator is changed to

$$
\begin{aligned}
& T_{a b}^{0}(f, g, h)(x) \\
= & \left(\int_{\mathbb{R}^{6}} a_{0}(\xi, \eta, \zeta) b_{0}(\eta, \zeta) \hat{f}(\xi) \hat{g}(\eta) \hat{h}(\zeta) e^{2 \pi i x(\xi+\eta+\zeta)} d \xi d \eta d \zeta\right) \varphi_{0}\left(x_{1}\right) \varphi_{0}^{\prime}\left(x_{2}\right) \\
= & \left(\int_{\mathbb{R}^{6}}(E+G)\left(E^{\prime}+G^{\prime}\right) \hat{f}(\xi) \hat{g}(\eta) \hat{h}(\zeta) e^{2 \pi i x(\xi+\eta+\zeta)} d \xi d \eta d \zeta\right) \varphi_{0}(x) \varphi_{0}^{\prime}\left(x_{2}\right) \\
:= & T_{a b}^{E, E^{\prime}, 0}+T_{a b}^{E, G^{\prime}, 0}+T_{a b}^{G, E^{\prime}, 0}+T_{a b}^{G, G^{\prime}, 0} .
\end{aligned}
$$

6.1. Estimates for $T_{a b}^{G, G^{\prime}, 0}$.

First consider $T_{a b}^{G, G^{\prime}, 0}$, recall

$$
\begin{gathered}
T_{a b}^{G, G^{\prime}, 0}(f, g, h)(x) \\
=\left(\int_{\mathbb{R}^{6}} m_{G, G^{\prime}}^{0}(\xi, \eta, \zeta) \hat{f}(\xi) \hat{g}(\eta) \hat{h}(\zeta) e^{2 \pi i x(\xi+\eta+\zeta)} d \xi d \eta d \zeta\right) \varphi_{0}\left(x_{1}\right) \varphi_{0}^{\prime}\left(x_{2}\right),
\end{gathered}
$$

where $m_{G, G^{\prime}}^{0}(\xi, \eta, \zeta):=G G^{\prime}$ satisfies

$$
\lesssim \frac{\left|\partial_{\xi_{1}, \xi_{2}}^{\alpha_{1}, \alpha_{2}} \partial_{\eta_{1}, \eta_{2}}^{\beta_{1}, \beta_{2}} \partial_{\zeta_{1}, \zeta_{2}}^{\gamma_{1}, \gamma_{2}} m_{G, G^{\prime}}^{0}(\xi, \eta, \zeta)\right|}{\left(1+\left|\xi_{1}\right|+\left|\eta_{1}\right|+\left|\zeta_{1}\right|\right)^{\alpha_{1}+\beta_{1}+\gamma_{1}}} \frac{1}{\left(1+\left|\xi_{2}\right|+\left|\eta_{2}\right|+\left|\zeta_{2}\right|\right)^{\alpha_{2}+\beta_{2}+\gamma_{2}}}
$$

for sufficiently many indices $\alpha_{1}, \alpha_{2}, \beta_{1}, \beta_{2}, \gamma_{1}, \gamma_{2}$. Then our desired localized estimate

$$
\left\|T_{a b}^{G, G^{\prime}, 0}(f, g, h)\right\|_{r} \lesssim\left\|f \tilde{\chi}_{R_{00}}\right\|_{p_{1}}\left\|g \tilde{\chi}_{R_{00}}\right\|_{p_{2}}\left\|h \tilde{\chi}_{R_{00}}\right\|_{p_{3}}
$$

follows from the proof of Theorem [1.5, see [7,20].

\subsection{Estimates for $T_{a b}^{E, E^{\prime}, 0}$.}

Recall

$$
E \cdot E^{\prime}=\left(\sum_{k_{1}} \widehat{\phi_{k_{1}}^{1}}\left(\xi_{1}\right) \widehat{\phi_{k_{1}}^{2}}\left(\eta_{1}\right) \widehat{\phi_{k_{1}}^{3}}\left(\zeta_{1}\right) \sum_{k_{2} \ll k_{1}} \widehat{\phi_{k_{2}}^{1}}\left(\eta_{1}\right) \widehat{\phi_{k_{2}}^{2}}\left(\zeta_{1}\right)\right)
$$




$$
\cdot\left(\sum_{k_{1}^{\prime}} \widehat{\phi_{k_{1}^{\prime}}^{1}}\left(\xi_{2}\right) \widehat{\phi_{k_{1}^{\prime}}^{2}}\left(\eta_{2}\right) \widehat{\phi_{k_{1}^{\prime}}^{3}}\left(\zeta_{2}\right) \sum_{k_{2}^{\prime} \ll k_{1}^{\prime}} \widehat{\phi_{k_{2}^{\prime}}^{1}}\left(\eta_{2}\right) \widehat{\phi_{k_{2}^{\prime}}^{2}}\left(\zeta_{2}\right)\right),
$$

where for each $l=k_{1}, k_{2}, k_{1}^{\prime}, k_{2}^{\prime}$, at least one of the families $\left(\widehat{\phi}_{l}^{1}\right)_{l}$ and $\left(\widehat{\phi}_{l}^{2}\right)_{l}$ is $\Psi$ type. And

$T_{a b}^{E, E^{\prime}, 0}(f, g, h)(x)=\left(\int_{\mathbb{R}^{6}}\left(E \cdot E^{\prime}\right) e^{2 \pi i x(\xi+\eta+\zeta)} \hat{f}(\xi) \hat{g}(\eta) \hat{h}(\zeta) d \xi d \eta d \zeta\right) \varphi_{0}\left(x_{1}\right) \varphi_{0}^{\prime}\left(x_{2}\right)$,

where we have removed multipliers $a_{0}, b_{0}$ by using Fourier series as before.

We now give two lemmas for single parameter operators when $x, \xi, \eta, \zeta \in \mathbb{R}$, which will be used later.

$$
\begin{gathered}
T^{E}(f, g, h)(x) \cdot \varphi_{0}(x)=: T_{a b}^{E, 0}(f, g, h)(x):= \\
\left(\int_{\mathbb{R}^{3}}\left(\sum_{k_{1}} \widehat{\phi_{k_{1}}^{1}}(\xi) \widehat{\phi_{k_{1}}^{2}}(\eta) \widehat{\phi_{k_{1}}^{3}}(\zeta)\right)\left(\sum_{k_{2} \ll k_{1}} \widehat{\phi_{k_{2}}^{1}}(\eta) \widehat{\phi_{k_{2}}^{2}}(\zeta)\right) \hat{f}(\xi) \hat{g}(\eta) \hat{h}(\zeta) e^{2 \pi i x(\xi+\eta+\zeta)} d \xi d \eta d \zeta\right) \varphi_{0}(x),
\end{gathered}
$$

where $x, \xi, \eta, \zeta \in \mathbb{R}$. From [18, 20], we can show $T^{E}$ can be decomposed into paraproducts. Before we state this result, we introduce some notations which are needed in the statement of the result.

Then we introduce some notations that will appear in the next lemma.

(a) We take $T_{1}(f, g, h)$ and $B_{I}^{1}(g, h)$ from (2.2) and (2.3) in Definition 5 .

(b) For positive integers $l$ and $k_{0} \geq 100$, let

$$
\begin{aligned}
& T_{l, k_{0}}^{1}(f, g, h)=\sum_{I \in \mathcal{I}} \frac{1}{|I|^{\frac{1}{2}}}\left\langle f, \phi_{I}^{l, 1}\right\rangle\left\langle B_{I, k_{0}}^{1, l}(g, h), \phi_{I}^{l, 2}\right\rangle \phi_{I}^{l, 3}
\end{aligned}
$$

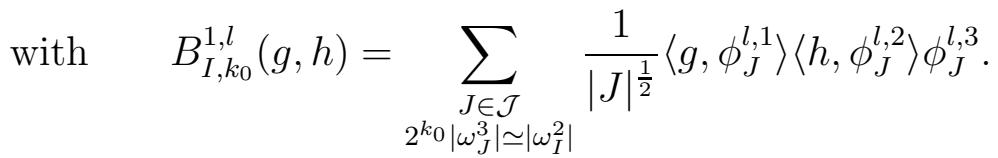

The functions $\left(\phi_{I}^{l, k}\right)_{k=1,2,3}$ in $T_{l, k_{0}}^{1}(f, g, h)$ and the functions $\left(\phi_{I}^{k}\right)_{k=1,2,3}$ in (2.4) are of the same "types" (whether each one is lacunary or non-lacunary), and so are the functions $\left(\phi_{J}^{l, k}\right)_{k=1,2,3}$ in (6.3) and the functions $\left(\phi_{J}^{k}\right)_{k=1,2,3}$ in(2.5) . Note the only difference between them is the dependence on $l$. In fact, $\phi_{I}^{l, 2}(x)$ could be $\left(\widehat{\phi}_{I}^{2}(\xi) \xi^{l}\right)^{\vee}$, but fortunately, this does not change the "types" of those functions. In this sense, $l$ will not play an important role in our estimates. For simplification, we omit this dependence on $l$ for all the expressions in the rest of the work.

(c) For a large positive integer $M$, let $T_{M, k_{0}}^{1}$ be a Fourier multiplier operator with $m_{M, k_{0}}^{1}(\xi, \eta, \zeta)$ satisfying the condition

$$
\left|\partial_{\xi}^{\alpha} \partial_{\eta}^{\beta} \partial_{\zeta}^{\gamma} m_{M, k_{0}}^{1}(\xi, \eta, \zeta)\right| \lesssim\left(2^{k_{0}}\right)^{\alpha+\beta+\gamma} \frac{1}{(1+|\xi|+|\eta|+|\zeta|)^{\alpha+\beta+\gamma}}
$$


for sufficiently many indices $\alpha, \beta, \gamma$.

(d) For $T_{1}$ and $T_{l, k_{0}}^{1}$ in $(a),(b)$, all the dyadic intervals have lengths at most 1 for all $k_{0} \geq 100,1 \leq l \leq M-1$.

With these notations, we are ready to state the following

Lemma 6.1. Define $T^{E}$ as in (6.2), then we can write

$$
\begin{gathered}
T^{E}(f, g, h)(x)= \\
T_{1}(f, g, h)(x)+\sum_{l=1}^{M-1} \sum_{k_{0}=100}^{\infty}\left(2^{-k_{0}}\right)^{l} T_{l, k_{0}}^{1}(f, g, h)(x)+\sum_{k_{0}=100}^{\infty}\left(2^{-k_{0}}\right)^{M} T_{M, k_{0}}^{1}(f, g, h)(x) .
\end{gathered}
$$

Proof. One can follow the work [18] closely, where the Taylor expansions of proper functions are used to get such forms of paraproducts. The only two statements we need to show are that all the dyadic intervals there have lengths at most one and the decay number 1 in the denominator from (6.4). In fact both of them follow from the fact $k_{1}, k_{2} \geq 0$ in (6.2).

Some more remarks for $T_{l, k_{0}}^{1}$ and $T_{M, k_{0}}^{1}$ are given below.

\section{Remark 2.}

(a) $T_{l, k_{0}}^{1}$ : For each $k_{0}$, one can see $T_{l, k_{0}}^{1}$ and $T_{1}$ are defined in very similar forms, and that means $T_{l, k_{0}}^{1}$ can be treated in the same way as $T_{1}$, since what really matters in the proof is the forms of paraproducts. More precisely, the bound of $T_{l, k_{0}}^{1}$ is actually independent of $k_{0}$, and then the factor $2^{-k_{0} l}$ allows us to take the summation over $k_{0}$. Thus, we will only deal with $T_{1}$ here, and one can easily get a similar argument for the $T_{l, k_{0}}^{1}$ part.

(b) $T_{M, k_{0}}^{1}$ : the condition (6.4) actually guarantees the estimate

$$
\left\|T_{M, k_{0}}^{1}(f, g, h)\right\|_{r} \lesssim 2^{100 k_{0}}\|f\|_{p_{1}}\|g\|_{p_{2}}\|h\|_{p_{3}},
$$

see [20]. By picking $M$ to be sufficiently large, we are able to take the summation over $k_{0}$ for $\left(2^{-k_{0}}\right)^{M} T_{M, k_{0}}^{1}(f, g, h)(x)$.

Now we return to the bi-parameter case. First consider $T_{a b}^{E, E^{\prime}, 0}$. Combining the ideas and the proof in those two lemmas (see [7, 18, 20] for details), one can check

$$
\begin{gathered}
T_{a b}^{E, E^{\prime}, 0}(f, g, h)(x) \\
\approx T_{1,1}^{E, E^{\prime}, 0}(f, g, h)(x)+T_{1, m_{M, k_{0}}^{\prime, 1}}^{E, E^{\prime}, 0}(f, g, h)(x) \\
+T_{m_{M, k_{0}}^{1}, E^{\prime}, 0}^{E}(f, g, h)(x)+T_{m_{M, k_{0}}^{1}, m_{M, k_{0}}^{\prime}, E^{\prime}, 0}^{1}(f, g, h)(x),
\end{gathered}
$$

where the definitions of the four operators will be given below. 
First let's see the simplest case, which is

$$
\begin{gathered}
T_{m_{M, k_{0}}^{1}, m_{M, k_{0}}^{\prime \prime, 1}}^{E, E^{\prime}, 0}(f, g, h)(x)= \\
\left(\int_{\mathbb{R}^{6}} m_{M, k_{0}}^{1}\left(\xi_{1}, \eta_{1}, \zeta_{1}\right) m_{M, k_{0}}^{\prime, 1}\left(\xi_{2}, \eta_{2}, \zeta_{2}\right) e^{2 \pi i x(\xi+\eta+\zeta)} \hat{f}(\xi) \hat{g}(\eta) \hat{h}(\zeta) d \xi d \eta d \zeta\right) \varphi_{0}\left(x_{1}\right) \varphi_{0}^{\prime}\left(x_{2}\right),
\end{gathered}
$$

where the symbols $m_{M, k_{0}}^{1}, m_{M, k_{0}}^{\prime, 1}$ are defined as in Lemma 6.1. In this case we can ignore $k_{0}$ as discussed in Remark 2 and clearly the desired estimate follows from Theorem 1.5.

Then we consider $T_{1,1}^{E, E^{\prime}, 0}(f, g, h)$.

$$
\begin{gathered}
T_{1,1}^{E, E^{\prime}, 0}(f, g, h):= \\
\left(\sum_{\substack{I \in \mathcal{I} \\
I^{\prime} \in \mathcal{I}^{\prime}}} \frac{1}{|I|^{\frac{1}{2}}} \frac{1}{\left|I^{\prime}\right| \frac{1}{2}}\left\langle f, \phi_{I}^{1} \otimes \phi_{I^{\prime}}^{1}\right\rangle\left\langle B_{I, I^{\prime}}^{1}(g, h), \phi_{I}^{2} \otimes \phi_{I^{\prime}}^{2}\right\rangle \phi_{I}^{3} \otimes \phi_{I^{\prime}}^{3}\right) \varphi_{0}\left(x_{1}\right) \varphi_{0}^{\prime}\left(x_{2}\right)
\end{gathered}
$$

with

$$
B_{I, I^{\prime}}^{1}(g, h)=\sum_{\substack{J \in \mathcal{J}, J \in \mathcal{J}^{\prime} \\\left|\omega_{J}^{3}\right| \leq\left|\omega_{I}^{2}\right| \\\left|\omega_{J^{\prime}}^{3}\right| \leq\left|\omega_{I^{\prime}}^{2}\right|}} \frac{1}{|J|^{\frac{1}{2}}} \frac{1}{\left|J^{\prime}\right|^{\frac{1}{2}}}\left\langle g, \phi_{J}^{1} \otimes \phi_{J^{\prime}}^{1}\right\rangle\left\langle h, \phi_{J}^{2} \otimes \phi_{J^{\prime}}^{2}\right\rangle \phi_{J}^{3} \otimes \phi_{J^{\prime}}^{3},
$$

where the families $\left(\phi_{I}^{j}\right)_{I \in \mathcal{I}},\left(\phi_{I^{\prime}}^{j}\right)_{I^{\prime} \in \mathcal{I}^{\prime}}(j=1,2,3)$ are defined as $\left(\phi_{I}^{j}\right)_{I \in \mathcal{I}}$, and the families $\left(\phi_{J}^{j}\right)_{I \in \mathcal{J}},\left(\phi_{J^{\prime}}^{j}\right)_{J^{\prime} \in \mathcal{J}^{\prime}}$ are defined as $\left(\phi_{J}^{j}\right)_{J \in \mathcal{J}}$ in Definition 5. Taking advantage of that $|I|,\left|I^{\prime}\right| \leq 1$, we can split

$$
\begin{gathered}
T_{1,1}^{E, E^{\prime}, 0}(f, g, h)=\left\{\left(\sum_{\substack{I \subseteq 5 I^{0} \\
I^{\prime} \subseteq 5 I^{0}}}+\sum_{\substack{I \subseteq\left(5 I^{0}\right)^{c} \\
I^{\prime} \subseteq 5 I^{0}}}+\sum_{\substack{I \subseteq 5 I^{0} \\
I^{\prime} \subseteq\left(5 I^{0}\right)^{c}}}+\sum_{\substack{I \subseteq\left(5 I^{0}\right)^{c} \\
I^{\prime} \subseteq\left(5 I^{0}\right)^{c}}}\right)\right. \\
\left.\frac{1}{|I|^{\frac{1}{2}}} \frac{1}{\left|I^{\prime}\right|^{\frac{1}{2}}}\left\langle f, \phi_{I}^{1} \otimes \phi_{I^{\prime}}^{1}\right\rangle\left\langle B_{I, I^{\prime}}^{1}(g, h), \phi_{I}^{2} \otimes \phi_{I^{\prime}}^{2}\right\rangle \phi_{I}^{3} \otimes \phi_{I^{\prime}}^{3}\right\} \varphi_{0}\left(x_{1}\right) \varphi_{0}^{\prime}\left(x_{2}\right) \\
=T_{1,1}^{E_{1}, E_{1}^{\prime}, 0}(f, g, h)+T_{1,1}^{E_{2}, E_{1}^{\prime}, 0}(f, g, h)+T_{1,1}^{E_{1}, E_{2}^{\prime}, 0}(f, g, h)+T_{1,1}^{E_{2}, E_{2}^{\prime}, 0}(f, g, h) .(6.5)
\end{gathered}
$$

We start with $T_{1,1}^{E_{1}, E_{1}^{\prime}, 0}(f, g, h)$. Consider the following decompositions

$$
\begin{gathered}
f(x)=\sum_{n_{1}, n_{1}^{\prime} \in \mathbb{Z}} f \chi_{I_{n_{1}}}\left(x_{1}\right) \chi_{I_{n_{1}^{\prime}}}\left(x_{2}\right), \quad g(x)=\sum_{n_{2}, n_{2}^{\prime} \in \mathbb{Z}} g \chi_{I_{n_{2}}}\left(x_{1}\right) \chi_{I_{n_{2}^{\prime}}}\left(x_{2}\right), \\
h(x)=\sum_{n_{3}, n_{3}^{\prime} \in \mathbb{Z}} h \chi_{I_{n_{3}}}\left(x_{1}\right) \chi_{I_{n_{3}^{\prime}}}\left(x_{2}\right) .
\end{gathered}
$$

Then we can write

$$
T_{1,1}^{E_{1}, E_{1}^{\prime}, 0}(f, g, h)(x)=
$$


$\sum_{n_{1}, n_{1}^{\prime}} \sum_{n_{2}, n_{2}^{\prime}} \sum_{n_{3}, n_{3}^{\prime}} T_{1,1}^{E_{1}, E_{1}^{\prime}, 0}\left(f \cdot \chi_{I_{n_{1}}} \otimes \chi_{I_{n_{1}^{\prime}}}, g \cdot \chi_{I_{n_{2}}} \otimes \chi_{I_{n_{2}^{\prime}}}, h \cdot \chi_{I_{n_{3}}} \otimes \chi_{I_{n_{3}^{\prime}}}\right)(x)$.

When $\left|n_{1}\right|,\left|n_{1}^{\prime}\right|>10$, we write

$$
\begin{aligned}
& \left\|T_{1,1}^{E_{1}, E_{1}^{\prime}, 0}\left(f \cdot \chi_{I_{n_{1}}} \otimes \chi_{I_{n_{1}^{\prime}}}, g \cdot \chi_{I_{n_{2}}} \otimes \chi_{I_{n_{2}^{\prime}}}, h \cdot \chi_{I_{n_{3}}} \otimes \chi_{I_{n_{3}^{\prime}}}\right)(x)\right\|_{r} \\
& =\| \sum_{I \in \mathcal{I}, I^{\prime} \in \mathcal{I}^{\prime}} \sum_{\substack{J \in \mathcal{J}, J^{\prime} \in \mathcal{J}^{\prime} \\
\left|\omega_{J}^{3}\right| \leq\left|\omega_{I}^{2}\right| \\
\left|\omega_{J^{\prime}}^{3}\right| \leq\left|\omega_{I^{\prime}}^{2}\right|}} \frac{1}{|I|^{\frac{1}{2}}} \frac{1}{|J|^{\frac{1}{2}}} \frac{1}{\left|I^{\prime}\right|^{\frac{1}{2}}} \frac{1}{\left|J^{\prime}\right|^{\frac{1}{2}}} \\
& \cdot\left\langle f \chi_{I_{n_{1}}} \otimes \chi_{I_{n_{1}^{\prime}}}, \phi_{I}^{1} \otimes \phi_{I^{\prime}}^{1}\right\rangle\left\langle g \chi_{I_{n_{2}}} \otimes \chi_{I_{n_{2}^{\prime}}}, \phi_{J}^{1} \otimes \phi_{J^{\prime}}^{1}\right\rangle\left\langle h \chi_{I_{n_{3}}} \otimes \chi_{I_{n_{3}^{\prime}}}, \phi_{J}^{2} \otimes \phi_{J^{\prime}}^{2}\right\rangle \\
& \cdot\left\langle\phi_{I}^{2} \otimes \phi_{I^{\prime}}^{2}, \phi_{J}^{3} \otimes \phi_{J^{\prime}}^{3}\right\rangle \phi_{I}^{3}\left(x_{1}\right) \phi_{I^{\prime}}^{3}\left(x_{2}\right) \varphi_{0}\left(x_{1}\right) \varphi_{0}^{\prime}\left(x_{2}\right) \|_{r} .
\end{aligned}
$$

Then we use Hölder's inequality to get

$$
\begin{aligned}
& \| \frac{1}{|I|^{\frac{1}{2}}} \frac{1}{|J|^{\frac{1}{2}}} \frac{1}{\left|I^{\prime}\right|^{\frac{1}{2}}} \frac{1}{\left|J^{\prime}\right|^{\frac{1}{2}}}\left\langle f \chi_{I_{n_{1}}} \otimes \chi_{I_{n_{1}^{\prime}}}, \phi_{I}^{1} \otimes \phi_{I^{\prime}}^{1}\right\rangle\left\langle g \chi_{I_{n_{2}}} \otimes \chi_{I_{n_{2}^{\prime}}}, \phi_{J}^{1} \otimes \phi_{J^{\prime}}^{1}\right\rangle \\
& \cdot\left\langle h \chi_{I_{n_{3}}} \otimes \chi_{I_{n_{3}^{\prime}}}, \phi_{J}^{2} \otimes \phi_{J^{\prime}}^{2}\right\rangle\left\langle\phi_{I}^{2} \otimes \phi_{I^{\prime}}^{2}, \phi_{J}^{3} \otimes \phi_{J^{\prime}}^{3}\right\rangle \phi_{I}^{3}\left(x_{1}\right) \phi_{I^{\prime}}^{3}\left(x_{2}\right) \varphi_{0}\left(x_{1}\right) \varphi_{0}^{\prime}\left(x_{2}\right) \|_{r} \\
& \lesssim \frac{1}{|I|^{2}} \frac{1}{|J|^{2}} \frac{1}{\left|I^{\prime}\right|^{2}} \frac{1}{\left|J^{\prime}\right|^{2}} \\
& \cdot\left(1+\frac{\operatorname{dist}\left(I_{n_{1}}, I\right)}{|I|}\right)^{-M_{1}}\left(1+\frac{\operatorname{dist}\left(I_{n_{1}^{\prime}}, I^{\prime}\right)}{\left|I^{\prime}\right|}\right)^{-M_{1}^{\prime}}\left(\left\|f \chi_{I_{n_{1}}} \otimes \chi_{I_{n_{1}^{\prime}}}\right\|_{p_{1}}\left(|I|\left|I^{\prime}\right|\right)^{\frac{p_{1}-1}{p_{1}}}\right) \\
& \cdot\left(1+\frac{\operatorname{dist}\left(I_{n_{2}}, J\right)}{|J|}\right)^{-N_{1}}\left(1+\frac{\operatorname{dist}\left(I_{n_{2}^{\prime}}, J^{\prime}\right)}{\left|J^{\prime}\right|}\right)^{-N_{1}^{\prime}}\left(\left\|g \chi_{I_{n_{2}}} \otimes \chi_{I_{n_{2}}^{\prime}}\right\|_{p_{2}}\left(\left|J \| J^{\prime}\right|\right)^{\frac{p_{2}-1}{p_{2}}}\right) \\
& \cdot\left(1+\frac{\operatorname{dist}\left(I_{n_{3}}, J\right)}{|J|}\right)^{-N_{2}}\left(1+\frac{\operatorname{dist}\left(I_{n_{3}^{\prime}}, J^{\prime}\right)}{\left|J^{\prime}\right|}\right)^{-N_{2}^{\prime}}\left(\left\|h \chi_{I_{n_{3}}} \otimes \chi_{I_{n_{3}^{\prime}}}\right\|_{p_{3}}\left(|J|\left|J^{\prime}\right|\right)^{\frac{p_{3}-1}{p_{3}}}\right) \\
& \cdot\left(|I|\left|I^{\prime}\right|\right)^{\frac{1}{r}} \int_{\mathbb{R}^{2}}\left(1+\frac{\operatorname{dist}\left(x_{1}, I\right)}{|I|}\right)^{-M_{2}}\left(1+\frac{\operatorname{dist}\left(x_{1}, J\right)}{|J|}\right)^{-N_{3}} \\
& \cdot\left(1+\frac{\operatorname{dist}\left(x_{2}, I^{\prime}\right)}{\left|I^{\prime}\right|}\right)^{-M_{2}^{\prime}}\left(1+\frac{\operatorname{dist}\left(x_{2}, J^{\prime}\right)}{\left|J^{\prime}\right|}\right)^{-N_{3}^{\prime}} d x \\
& \lesssim \frac{1}{|I|\left|I^{\prime}\right|}\left(\frac{|I|\left|I^{\prime}\right|}{|J|\left|J^{\prime}\right|}\right)^{\frac{1}{p_{2}}+\frac{1}{p_{3}}}\left(1+\frac{\operatorname{dist}\left(I_{n_{1}}, I\right)}{|I|}\right)^{-M_{1}}\left(1+\frac{\operatorname{dist}\left(I_{n_{1}^{\prime}}, I^{\prime}\right)}{\left|I^{\prime}\right|}\right)^{-M_{1}^{\prime}} \\
& \cdot\left(1+\frac{\operatorname{dist}\left(I_{n_{2}}, J\right)}{|J|}\right)^{-N_{1}}\left(1+\frac{\operatorname{dist}\left(I_{n_{2}^{\prime}}, J^{\prime}\right)}{\left|J^{\prime}\right|}\right)^{-N_{1}^{\prime}} \\
& \cdot\left(1+\frac{\operatorname{dist}\left(I_{n_{3}}, J\right)}{|J|}\right)^{-N_{2}}\left(1+\frac{\operatorname{dist}\left(I_{n_{3}^{\prime}}, J^{\prime}\right)}{\left|J^{\prime}\right|}\right)^{-N_{2}^{\prime}}
\end{aligned}
$$




$$
\begin{aligned}
& \cdot \int_{\mathbb{R}^{2}}\left(1+\frac{\operatorname{dist}\left(x_{1}, I\right)}{|I|}\right)^{-M_{2}}\left(1+\frac{\operatorname{dist}\left(x_{1}, J\right)}{|J|}\right)^{-N_{3}} \\
& \cdot\left(1+\frac{\operatorname{dist}\left(x_{2}, I^{\prime}\right)}{\left|I^{\prime}\right|}\right)^{-M_{2}^{\prime}}\left(1+\frac{\operatorname{dist}\left(x_{2}, J^{\prime}\right)}{\left|J^{\prime}\right|}\right)^{-N_{3}^{\prime}} d x \\
& \cdot\left\|f \chi_{I_{n_{1}}} \otimes \chi_{I_{n_{1}^{\prime}}}\right\|_{p_{1}}\left\|g \chi_{I_{n_{2}}} \otimes \chi_{I_{n_{2}^{\prime}}}\right\|_{p_{2}}\left\|h \chi_{I_{n_{3}}} \otimes \chi_{I_{n_{3}^{\prime}}}\right\|_{p_{3}},
\end{aligned}
$$

where $M_{j}, M_{j}^{\prime}, N_{j}, N_{j}^{\prime}$ are sufficiently large integers and $\phi_{I}^{j}, \phi_{J}^{j}, \phi_{I^{\prime}}^{j}, \phi_{J^{\prime}}^{j}$ are $L^{2}$-normalized bump functions adapted to $I, I^{\prime}, J, J^{\prime}$ for $j=1,2,3$.

Then we use the fact that $\left|\omega_{J}^{3}\right| \leq\left|\omega_{I}^{2}\right|,\left|\omega_{J^{\prime}}^{3}\right| \leq\left|\omega_{I^{\prime}}^{2}\right|$, which implies $|I| \lesssim|J|,\left|I^{\prime}\right| \lesssim$ $\left|J^{\prime}\right|$ and take advantage of the locations of dyadic intervals $J$ as well. Using the notation $J_{m}=[m, m+1], m \in \mathbb{Z}$ and (6.7) we can get for $0<r<1(r>1$ will be similar, and from now on we always assume $0<r<1$ )

$$
\begin{aligned}
& \left\|T_{1,1}^{E_{1}, E_{1}^{\prime}, 0}\left(f \cdot \chi_{I_{n_{1}}} \otimes \chi_{I_{n_{1}^{\prime}}}, g \cdot \chi_{I_{n_{2}}} \otimes \chi_{I_{n_{2}^{\prime}}}, h \cdot \chi_{I_{n_{3}}} \otimes \chi_{I_{n_{3}^{\prime}}}\right)(x)\right\|_{r}^{r} \\
& \lesssim \sum_{\substack{i, i^{\prime} \geq 0 \\
j, j^{\prime} \geq 0}} \sum_{\substack{I, I^{\prime} \subseteq 5 I^{0} \\
|I|=2^{-i} \\
\left|I^{\prime}\right|=2^{-i^{\prime}}}} \sum_{m, m^{\prime} \in \mathbb{Z}} \sum_{\substack{J \subseteq J_{m},|J|=2^{-j} \\
J^{\prime} \subseteq J_{m^{\prime}},\left|J^{\prime}\right|=2^{-j^{\prime}}}}\left(\frac{1}{|I|\left|I^{\prime}\right|}\left(1+\frac{\operatorname{dist}\left(I_{n_{1}}, I\right)}{|I|}\right)^{-M_{1}}\left(1+\frac{\operatorname{dist}\left(I_{n_{1}^{\prime}}, I\right)}{|I|}\right)^{-M_{1}^{\prime}}\right. \\
& \left(1+\frac{\operatorname{dist}\left(I_{n_{2}}, J\right)}{|J|}\right)^{-N_{1}}\left(1+\frac{\operatorname{dist}\left(I_{n_{2}^{\prime}}, J^{\prime}\right)}{\left|J^{\prime}\right|}\right)^{-N_{1}^{\prime}} \\
& \cdot\left(1+\frac{\operatorname{dist}\left(I_{n_{3}}, J\right)}{|J|}\right)^{-N_{2}}\left(1+\frac{\operatorname{dist}\left(I_{n_{3}^{\prime}}, J^{\prime}\right)}{\left|J^{\prime}\right|}\right)^{-N_{2}^{\prime}} \\
& \cdot \int_{\mathbb{R}^{2}}\left(1+\frac{\operatorname{dist}\left(x_{1}, I\right)}{|I|}\right)^{-M_{2}}\left(1+\frac{\operatorname{dist}\left(x_{2}, I^{\prime}\right)}{\left|I^{\prime}\right|}\right)^{-M_{2}^{\prime}} \\
& \cdot\left(1+\frac{\operatorname{dist}\left(x_{1}, J\right)}{|J|}\right)^{-N_{3}}\left(1+\frac{\operatorname{dist}\left(x_{2}, J^{\prime}\right)}{\left|J^{\prime}\right|}\right)^{-N_{3}^{\prime}} d x \\
& \left.\cdot\left\|f \chi_{I_{n_{1}}} \otimes \chi_{I_{n_{1}^{\prime}}}\right\|_{p_{1}}\left\|g \chi_{I_{n_{2}}} \otimes \chi_{I_{n_{2}^{\prime}}}\right\|_{p_{2}}\left\|h \chi_{I_{n_{3}}} \otimes \chi_{I_{n_{3}^{\prime}}}\right\|_{p_{3}}\right)^{r} \\
& \lesssim \sum_{\substack{i, i^{\prime} \geq 0 \\
j, j^{\prime} \subseteq 0}} \sum_{\substack{I, I^{\prime} \subseteq 5 I_{0} \\
|I|=I^{-i} \\
\left|I^{\prime}\right|=2^{-i^{\prime}}}} \sum_{m, m^{\prime} \in \mathbb{Z}} \sum_{\substack{J \subseteq J_{m},|J|=2^{-j} \\
J^{\prime} \subseteq J_{m^{\prime}},\left|J^{\prime}\right|=2^{-j^{\prime}}}}\left(2^{i+i^{\prime}}\left(1+2^{i}\left(\left|n_{1}\right|-6\right)\right)^{-M_{1}}\left(1+2^{i^{\prime}}\left(\left|n_{1}^{\prime}\right|-6\right)\right)^{-M_{1}^{\prime}}\right. \\
& \cdot\left(1+2^{j}\left|m-n_{2}\right|\right)^{-N_{1}}\left(1+2^{j^{\prime}}\left|m^{\prime}-n_{2}^{\prime}\right|\right)^{-N_{1}^{\prime}}\left(1+2^{j}\left|m-n_{3}\right|\right)^{-N_{2}} \\
& \cdot\left(1+2^{j^{\prime}}\left|m^{\prime}-n_{3}^{\prime}\right|\right)^{-N_{2}^{\prime}}(1+|m|)^{-N_{0}}\left(1+\left|m^{\prime}\right|\right)^{-N_{0}^{\prime}} \\
& \left.\cdot\left\|f \chi_{I_{n_{1}}} \otimes \chi_{I_{n_{1}^{\prime}}}\right\|_{p_{1}}\left\|g \chi_{I_{n_{2}}} \otimes \chi_{I_{n_{2}^{\prime}}}\right\|_{p_{2}}\left\|h \chi_{I_{n_{3}}} \otimes \chi_{I_{n_{3}^{\prime}}}\right\|_{p_{3}}\right)^{r} \\
& \lesssim \sum_{m, m^{\prime} \in \mathbb{Z}}\left(\left(\left|n_{1}\right|-6\right)^{-\frac{M_{1}}{2}}\left(\left|n_{1}^{\prime}\right|-6\right)^{-\frac{M_{1}^{\prime}}{2}}\left(1+\left|n_{2}\right|\right)^{-L}\left(1+\left|n_{2}^{\prime}\right|\right)^{-L}\right.
\end{aligned}
$$




$$
\begin{aligned}
& \cdot\left(1+\left|n_{3}\right|\right)^{-L}\left(1+\left|n_{3}^{\prime}\right|\right)^{-L}(1+|m|)^{-\frac{N_{0}}{2}}\left(1+\left|m^{\prime}\right|\right)^{-\frac{N_{0}^{\prime}}{2}} \\
& \left.\cdot\left\|f \chi_{I_{n_{1}}} \otimes \chi_{I_{n_{1}^{\prime}}}\right\|_{p_{1}}\left\|g \chi_{I_{n_{2}}} \otimes \chi_{I_{n_{2}}}\right\|_{p_{2}}\left\|h \chi_{I_{n_{3}}} \otimes \chi_{I_{n_{3}^{\prime}}}\right\|_{p_{3}}\right)^{r}
\end{aligned}
$$

where the positive integers $N_{0}=\min \left\{M_{2}, N_{3}\right\}, N_{0}^{\prime}=\min \left\{M_{2}^{\prime}, N_{3}^{\prime}\right\}, L$ are sufficiently large and the last inequality holds since for any $l, m \in \mathbb{Z}$ and any large integer $M$, there exists a large integer $M^{\prime}$ such that

$$
(1+|l-m|)^{-M}(1+|m|)^{-\frac{N_{0}}{4}} \lesssim(1+|l|)^{-M^{\prime}} .
$$

And also note that in the above calculation we can take summation over $i, j, i^{\prime}, j^{\prime}$ because when $\left|n_{1}-6\right|,\left|n_{1}^{\prime}-6\right|>0$ the power $M_{1}, M_{1}^{\prime}$ can give a decay for $i, i^{\prime}$, and consequently a decay for $j, j^{\prime}$ as well since $i \gtrsim j \geq 0, i^{\prime} \gtrsim j^{\prime} \geq 0$.

Now we take the summation

$$
\begin{aligned}
& \left\|\sum_{\substack{\left|n_{1}\right|>10 \\
\left|n_{1}^{\prime}\right|>10}} \sum_{n_{2}, n_{3}, n_{2}^{\prime}, n_{3}^{\prime} \in \mathbb{Z}} T_{1,1}^{E_{1}, E_{1}^{\prime}, 0}\left(f \cdot \chi_{I_{n_{1}}} \otimes \chi_{I_{n_{1}^{\prime}}}, g \cdot \chi_{I_{n_{2}}} \otimes \chi_{I_{n_{2}^{\prime}}}, h \cdot \chi_{I_{n_{3}}} \otimes \chi_{I_{n_{3}^{\prime}}}\right)(x)\right\|_{r}^{r} \\
\lesssim & \sum_{\left|n_{1}\right|>10,\left|n_{1}^{\prime}\right|>10} \sum_{n_{2}, n_{3}, n_{2}^{\prime}, n_{3}^{\prime} \in \mathbb{Z}}\left(\left(\left|n_{1}\right|-6\right)^{-\frac{M_{1}}{2}}\left(\left|n_{1}^{\prime}\right|-6\right)^{-\frac{M_{1}^{\prime}}{2}}\left(1+\left|n_{2}\right|\right)^{-L}\left(1+\left|n_{2}^{\prime}\right|\right)^{-L}\right. \\
& \left.\cdot\left(1+\left|n_{3}\right|\right)^{-L}\left(1+\left|n_{3}^{\prime}\right|\right)^{-L}\left\|f \chi_{I_{n_{1}}} \otimes \chi_{I_{n_{1}^{\prime}}}\right\|_{p_{1}}\left\|g \chi_{I_{n_{2}}} \otimes \chi_{I_{n_{2}^{\prime}}}\right\|_{p_{2}}\left\|h \chi_{I_{n_{3}}} \otimes \chi_{I_{n_{3}^{\prime}}}\right\|_{p_{3}}\right)^{r} \\
\lesssim & \sum_{\left|n_{1}\right|>10,\left|n_{1}^{\prime}\right|>10} \sum_{n_{2}, n_{3}, n_{2}^{\prime}, n_{3}^{\prime} \in \mathbb{Z}}\left(\left(\left|n_{1}\right|-6\right)^{-\frac{M_{1}}{4}}\left(\left|n_{1}^{\prime}\right|-6\right)^{-\frac{M_{1}^{\prime}}{4}}\left(1+\left|n_{2}\right|\right)^{-\frac{L}{2}}\left(1+\left|n_{2}^{\prime}\right|\right)^{-\frac{L}{2}}\right. \\
& \left.\cdot\left(1+\left|n_{3}\right|\right)^{-\frac{L}{2}}\left(1+\left|n_{3}^{\prime}\right|\right)^{-\frac{L}{2}}\left\|f \tilde{\chi}_{R_{00}}\right\|_{p_{1}}\left\|g \tilde{\chi}_{R_{00}}\right\|_{p_{2}}\left\|h \tilde{\chi}_{R_{00}}\right\|_{p_{3}}\right)^{r} \\
\lesssim \quad & \left(\left\|f \tilde{\chi}_{R_{00}}\right\|_{p_{1}}\left\|g \tilde{\chi}_{R_{00}}\right\|_{p_{2}}\left\|h \tilde{\chi}_{R_{00}}\right\|_{p_{3}}\right)^{r},
\end{aligned}
$$

where we use the fact for any $n \in \mathbb{Z}$ and large integer $L$, there holds

$$
(1+|n|)^{-\frac{L}{2}} \cdot \chi_{I_{n}} \lesssim \tilde{\chi}_{I^{0}}
$$

When $\left|n_{1}\right| \leq 10$ or $\left|n_{1}^{\prime}\right| \leq 10$ things are different. Say $\left|n_{1}\right| \leq 10$, in this situation, the terms like $\left(1+\frac{\operatorname{dist}\left(I_{n_{1}}, I\right)}{|I|}\right)^{-M_{1}}$ in (6.7) won't give us a decay factor on $i$, which means we will have trouble taking the summation over dyadic intervals $I$. Actually the decay factors from other terms are with respect to $j$ which can't help since $i \gtrsim j$. And the same problem exists for $i^{\prime}, j^{\prime}$ as well. This is actually where such paraproducts behave differently from the classical ones. In the classical case only one class of dyadic intervals is involved, but here we have $I$ and $J, I^{\prime}$ and $J^{\prime}$, where the decay factors coming from either class might not be used for the other one. We will make use of Theorem 1.7 here. Without loss of generality, we assume both $\left|n_{1}\right| \leq 10$ and $\left|n_{1}^{\prime}\right| \leq 10$. Now the goal is 


$$
\begin{aligned}
&\left\|\sum_{\substack{\left|n_{1}\right|,\left|n_{n}^{\prime}\right| \leq 10 \\
n_{2}, n_{2}^{\prime}, n_{3}, n_{3}^{\prime} \in \mathbb{Z}}} T_{1,1}^{E_{1}, E_{1}^{\prime}, 0}\left(f \cdot \chi_{I_{n_{1}}} \otimes \chi_{I_{n_{1}^{\prime}}}, g \cdot \chi_{I_{n_{2}}} \otimes \chi_{I_{n_{2}^{\prime}}}, h \cdot \chi_{I_{n_{3}}} \otimes \chi_{I_{n_{3}^{\prime}}}\right)(x)\right\|_{r} \\
& \lesssim\left\|f \tilde{\chi}_{R_{00}}\right\|_{p_{1}}\left\|g \tilde{\chi}_{R_{00}}\right\|_{p_{2}}\left\|h \tilde{\chi}_{R_{00}}\right\|_{p_{3}} .
\end{aligned}
$$

Recall that when $I, I^{\prime} \subseteq 5 I^{0}$ and $J \in J_{m}, J^{\prime} \in J_{m^{\prime}}$, in (6.7) we can write

$$
\left\langle\phi_{I}^{2} \otimes \phi_{I^{\prime}}^{2}, \phi_{J}^{3} \otimes \phi_{J^{\prime}}^{3}\right\rangle \approx(1+|m|)^{-L}\left(1+\left|m^{\prime}\right|\right)^{-L}\left\langle\phi_{I}^{2} \otimes \phi_{I^{\prime}}^{2}, \tilde{\phi}_{J}^{3} \otimes \tilde{\phi}_{J^{\prime}}^{3}\right\rangle,
$$

$\left\langle g \chi_{I_{n_{2}}} \otimes \chi_{I_{n_{2}^{\prime}}}, \phi_{J}^{1} \otimes \phi_{J^{\prime}}^{1}\right\rangle \approx\left(1+\left|n_{2}-m\right|\right)^{-M}\left(1+\left|n_{2}^{\prime}-m^{\prime}\right|\right)^{-M}\left\langle g \chi_{I_{n_{2}}} \otimes \chi_{I_{n_{2}^{\prime}}}, \tilde{\phi}_{J}^{1} \otimes \tilde{\phi}_{J^{\prime}}^{1}\right\rangle$,

$\left\langle h \chi_{I_{n_{3}}} \otimes \chi_{I_{n_{3}^{\prime}}}, \phi_{J}^{2} \otimes \phi_{J^{\prime}}^{2}\right\rangle \approx\left(1+\left|n_{3}-m\right|\right)^{-M}\left(1+\left|n_{3}^{\prime}-m^{\prime}\right|\right)^{-M}\left\langle h \chi_{I_{n_{3}}} \otimes \chi_{I_{n_{3}^{\prime}}}, \tilde{\phi}_{J}^{2} \otimes \tilde{\phi}_{J^{\prime}}^{2}\right\rangle$,

where $\tilde{\phi}_{J}^{l}, \tilde{\phi}_{J^{\prime}}^{l}$ are properly chosen bump functions adapted to $J, J^{\prime}$ that have the same type as $\phi_{J}^{l}, \phi_{J^{\prime}}^{l}(l=1,2,3)$ respectively, and $L, M$ are sufficiently large integers. Also, by (6.8)

$$
\begin{aligned}
& \left(1+\left|n_{2}-m\right|\right)^{-M}\left(1+\left|n_{2}^{\prime}-m^{\prime}\right|\right)^{-M}(1+|m|)^{-L / 2}\left(1+\left|m^{\prime}\right|\right)^{-L / 2} \approx\left(1+\left|n_{2}\right|\right)^{-M^{\prime}} \cdot\left(1+\left|n_{2}^{\prime}\right|\right)^{-M^{\prime}}, \\
& \left(1+\left|n_{3}-m\right|\right)^{-M}\left(1+\left|n_{3}^{\prime}-m^{\prime}\right|\right)^{-M}(1+|m|)^{-L / 2}\left(1+\left|m^{\prime}\right|\right)^{-L / 2} \approx\left(1+\left|n_{3}\right|\right)^{-M^{\prime}} \cdot\left(1+\left|n_{3}^{\prime}\right|\right)^{-M^{\prime}},
\end{aligned}
$$

where $M^{\prime}$ can be sufficiently large.

That means when dealing with the paraproducts, we can go back to the original form of operators in Theorem [1.7, with additional decay factors $\left(1+\left|n_{2}\right|\right)^{-M^{\prime}}(1+$ $\left.\left|n_{2}^{\prime}\right|\right)^{-M^{\prime}} \cdot\left(1+\left|n_{3}\right|\right)^{-M^{\prime}}\left(1+\left|n_{3}^{\prime}\right|\right)^{-M^{\prime}}$. Thus,

$$
\begin{aligned}
& \left\|\sum_{\substack{\left|n_{1}\right|,\left|n_{1}^{\prime}\right| \leq 10 \\
n_{2}, n_{2}^{\prime}, n_{3}, n_{3}^{\prime} \in \mathbb{Z}}} T_{1,1}^{E_{1}, E_{1}^{\prime}, 0}\left(f \cdot \chi_{I_{n_{1}}} \otimes \chi_{I_{n_{1}^{\prime}}}, g \cdot \chi_{I_{n_{2}}} \otimes \chi_{I_{n_{2}^{\prime}}}, h \cdot \chi_{I_{n_{3}}} \otimes \chi_{I_{n_{3}^{\prime}}}\right)(x)\right\|_{r} \\
\lesssim & \sum_{\substack{\left|n_{1}\right|,\left|n_{1}^{\prime}\right| \leq 10 \\
n_{2}, n_{2}^{\prime}, n_{3}, n_{3}^{\prime} \in \mathbb{Z}\\
}}\left(1+\left|n_{2}\right|\right)^{-M^{\prime}}\left(1+\left|n_{2}^{\prime}\right|\right)^{-M^{\prime}} \cdot\left(1+\left|n_{3}\right|\right)^{-M^{\prime}}\left(1+\left|n_{3}^{\prime}\right|\right)^{-M^{\prime}} \\
\lesssim & \left\|f \cdot \chi_{I_{n_{1}}} \otimes \chi_{R_{00}}\right\|_{p_{1}}\left\|g \tilde{\chi}_{R_{00}}\right\|_{p_{1}}\left\|g \cdot \chi_{p_{2}}\right\| h \tilde{\chi}_{I_{n_{2}}} \otimes \chi_{I_{n_{2}^{\prime}}} \|_{p_{3}} .
\end{aligned}
$$

For the cases $n_{1} \leq 10, n_{1}^{\prime}>10$ or $n_{1}>10, n_{1}^{\prime} \leq 10$, one just needs to combine the ideas in the above two situations together and use Theorem 1.7. We omit the details and the case $T_{a b}^{E_{1}, E_{1}^{\prime}, 0}$ has been done, where $I, I^{\prime} \subseteq 5 I^{0}$.

Now we turn to the study of the operators $T_{1,1}^{E_{2}, E_{2}^{\prime}, 0}$.

$$
\left\|T_{1,1}^{E_{2}, E_{2}^{\prime}, 0}(f, g, h)(x)\right\|_{r}^{r}
$$


BI-PARAMETER TRILINEAR FOURIER MULTIPLIERS WITH FLAG SYMBOL

$$
\begin{aligned}
& =\left\|\sum_{\substack{I \subseteq\left(5 I^{0}\right)^{c} \\
I^{\prime} \subseteq\left(5 I^{\prime 0}\right)^{c}}} \frac{1}{|I|^{\frac{1}{2}}} \frac{1}{\left|I^{\prime}\right|^{\frac{1}{2}}}\left\langle f, \phi_{I}^{1} \otimes \phi_{I^{\prime}}^{1}\right\rangle\left\langle B_{I, I^{\prime}}^{1}(g, h), \phi_{I}^{2} \otimes \phi_{I^{\prime}}^{2}\right\rangle \phi_{I}^{3} \otimes \phi_{I^{\prime}}^{3} \varphi_{0}\left(x_{1}\right) \varphi_{0}^{\prime}\left(x_{2}\right)\right\|_{r}^{r} \\
& =\| \sum_{\substack{I \in \mathcal{I} \\
I^{\prime} \in \mathcal{I}^{\prime}}} \sum_{\substack{J \in \mathcal{J}, J^{\prime} \in \mathcal{J}^{\prime} \\
\left|\omega_{J}^{3}\right| \leq\left|\omega_{I}^{2}\right| \\
\left|\omega_{J^{\prime}}^{3}\right| \leq\left|\omega_{I^{\prime}}^{2}\right|}} \frac{1}{|I|^{\frac{1}{2}}} \frac{1}{|J|^{\frac{1}{2}}} \frac{1}{\left|I^{\prime}\right|^{\frac{1}{2}}} \frac{1}{\left|J^{\prime}\right|^{\frac{1}{2}}}\left\langle f, \phi_{I}^{1} \otimes \phi_{I^{\prime}}^{1}\right\rangle\left\langle g, \phi_{J}^{1} \otimes \phi_{J^{\prime}}^{1}\right\rangle \\
& \cdot\left\langle h, \phi_{J}^{2} \otimes \phi_{J^{\prime}}^{2}\right\rangle\left\langle\phi_{I}^{2} \otimes \phi_{I^{\prime}}^{2}, \phi_{J}^{3} \otimes \phi_{J^{\prime}}^{3}\right\rangle \phi_{I}^{3}\left(x_{1}\right) \phi_{I^{\prime}}^{3}\left(x_{2}\right) \varphi_{0}\left(x_{1}\right) \varphi_{0}^{\prime}\left(x_{2}\right) \|_{r}^{r}
\end{aligned}
$$

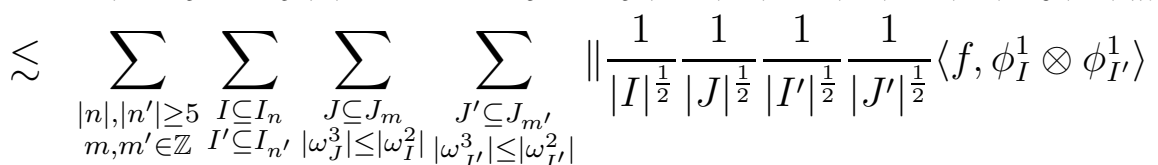

$$
\begin{aligned}
& \left\langle g, \phi_{J}^{1} \otimes \phi_{J^{\prime}}^{1}\right\rangle\left\langle h, \phi_{J}^{2} \otimes \phi_{J^{\prime}}^{2}\right\rangle\left\langle\phi_{I}^{2} \otimes \phi_{I^{\prime}}^{2}, \phi_{J}^{3} \otimes \phi_{J^{\prime}}^{3}\right\rangle \phi_{I}^{3}\left(x_{1}\right) \phi_{I^{\prime}}^{3}\left(x_{2}\right) \varphi_{0}\left(x_{1}\right) \varphi_{0}^{\prime}\left(x_{2}\right) \|_{r}^{r} .
\end{aligned}
$$

We use Hölder's inequality and take advantage of the decay factors as before

$$
\begin{aligned}
& \sum_{\substack{|n|,\left|n^{\prime}\right| \geq 5 \\
m, m^{\prime} \in \mathbb{Z}}} \sum_{\substack{i, j \geq 0 \\
i^{\prime}, j^{\prime} \geq 0}} \sum_{\substack{I \subseteq I_{n}, J \subseteq J_{m} \\
|I|=2^{-i},|J|=2^{-j}}} \sum_{\substack{I^{\prime} \subseteq I_{n^{\prime}, J^{\prime} \subseteq J_{m^{\prime}}} \\
\left|I^{\prime}\right|=2^{-i^{\prime}},\left|J^{\prime}\right|=2^{-j^{\prime}}}} \| \frac{1}{|I|^{\frac{1}{2}}} \frac{1}{|J|^{\frac{1}{2}}} \frac{1}{\left|I^{\prime}\right|^{\frac{1}{2}}} \frac{1}{\left|J^{\prime}\right|^{\frac{1}{2}}}\left\langle f, \phi_{I}^{1} \otimes \phi_{I^{\prime}}^{1}\right\rangle \\
& \left\langle g, \phi_{J}^{1} \otimes \phi_{J^{\prime}}^{1}\right\rangle\left\langle h, \phi_{J}^{2} \otimes \phi_{J^{\prime}}^{2}\right\rangle\left\langle\phi_{I}^{2} \otimes \phi_{I^{\prime}}^{2}, \phi_{J}^{3} \otimes \phi_{J^{\prime}}^{3}\right\rangle \phi_{I}^{3}\left(x_{1}\right) \phi_{I^{\prime}}^{3}\left(x_{2}\right) \varphi_{0}\left(x_{1}\right) \varphi_{0}^{\prime}\left(x_{2}\right) \|_{r}^{r} \\
& \lesssim \sum_{\substack{|n|,\left|n^{\prime}\right| \geq 5 \\
m, m^{\prime} \in \mathbb{Z}}} \sum_{\substack{i, j \geq 0 \\
i^{\prime}, j^{\prime} \geq 0}} \sum_{\substack{I \subseteq I_{n}, J \subseteq J_{m} \\
|I|=2^{-i},|J|=2^{-j}}} \sum_{\substack{\left.I^{\prime} \subseteq I_{n^{\prime}, J^{\prime} \subseteq J_{m^{\prime}}} I^{\prime}\left|=2^{-i^{\prime}},\right| J^{\prime}\left|=2^{-j^{\prime}}\\
\right| I\right|^{2}}}\left(\frac{1}{|J|^{2}} \frac{1}{\left|I^{\prime}\right|^{2}} \frac{1}{\left|J^{\prime}\right|^{2}}\right. \\
& \cdot\left(\left\|f \chi_{I_{n}} \otimes \chi_{I_{n^{\prime}}}\right\|_{p_{1}}\left(|I|\left|I^{\prime}\right|\right)^{\frac{p_{1}-1}{p_{1}}}\right)\left(\left\|g \chi_{J_{m}} \otimes \chi_{J_{m^{\prime}}}\right\|_{p_{2}}\left(|J|\left|J^{\prime}\right|\right)^{\frac{p_{2}-1}{p_{2}}}\right) \\
& \cdot\left(\left.\left\|h \chi_{J_{m}} \otimes \chi_{J_{m^{\prime}}}\right\|_{p_{3}}\left(|J| \mid J^{\prime}\right)\right|^{\frac{p_{3}-1}{p_{3}}}\right)\left(|I|\left|I^{\prime}\right|\right)^{\frac{1}{r}}\left(1+\frac{\operatorname{dist}\left(I, I^{0}\right)}{|I|}\right)^{-M_{3}} \\
& \cdot\left(1+\frac{\operatorname{dist}\left(I^{\prime}, I^{0}\right)}{\left|I^{\prime}\right|}\right)^{-M_{3}^{\prime}} \int_{\mathbb{R}^{2}}\left(1+\frac{\operatorname{dist}\left(x_{1}, I\right)}{|I|}\right)^{-M_{2}}\left(1+\frac{\operatorname{dist}\left(x_{2}, I^{\prime}\right)}{\left|I^{\prime}\right|}\right)^{-M_{2}^{\prime}} \\
& \left.\cdot\left(1+\frac{\operatorname{dist}\left(x_{1}, J\right)}{|J|}\right)^{-N_{3}}\left(1+\frac{\operatorname{dist}\left(x_{2}, J^{\prime}\right)}{\left|J^{\prime}\right|}\right)^{-N_{3}^{\prime}} d x\right)^{r} \\
& \lesssim \sum_{\substack{|n|,\left|n^{\prime}\right| \geq 5 \\
m, m^{\prime} \in \mathbb{Z}}} \sum_{\substack{i, j \geq 0 \\
i^{\prime}, j^{\prime} \geq 0}} \sum_{\substack{I \subseteq I_{n}, J \subseteq J_{m} \\
|I|=2^{-i},|J|=2^{-j}}} \sum_{\substack{I^{\prime} \subseteq I_{n^{\prime}}, J^{\prime} \subseteq J_{m^{\prime}} \\
\left|I^{\prime}\right|=2^{-i^{\prime}},\left|J^{\prime}\right|=2^{-j^{\prime}}}}\left(2^{i+i^{\prime}}\left(1+2^{i}(|n|-2)\right)^{-M_{3}}\right. \\
& \cdot\left(1+2^{i^{\prime}}\left(\left|n^{\prime}\right|-2\right)\right)^{-M_{3}^{\prime}}(1+|n-m|)^{-N_{0}}\left(1+\left|n^{\prime}-m^{\prime}\right|\right)^{-N_{0}^{\prime}} \\
& \left.\cdot\left\|f \chi_{I_{n}} \otimes \chi_{I_{n^{\prime}}}\right\|_{p_{1}}\left\|g \chi_{J_{m}} \otimes \chi_{J_{m^{\prime}}}\right\|_{p_{2}}\left\|\chi_{J_{m}} \otimes \chi_{J_{m^{\prime}}}\right\|_{p_{3}}\right)^{r} \\
& \lesssim \sum_{\substack{|n|,\left|n^{\prime}\right| \geq 5 \\
m, m^{\prime} \in \mathbb{Z}}}\left((|n|-2)^{\frac{-M_{3}}{2}}\left(\left|n^{\prime}\right|-2\right)^{\frac{-M_{3}^{\prime}}{2}}(1+|n-m|)^{-N_{0}}\left(1+\left|n^{\prime}-m^{\prime}\right|\right)^{-N_{0}^{\prime}}\right.
\end{aligned}
$$




$$
\left.\cdot\left\|f \chi_{I_{n}} \otimes \chi_{I_{n^{\prime}}}\right\|_{p_{1}}\left\|g \chi_{J_{m}} \otimes \chi_{J_{m^{\prime}}}\right\|_{p_{2}}\left\|h \chi_{J_{m}} \otimes \chi_{J_{m^{\prime}}}\right\|_{p_{3}}\right)^{r}
$$

where again $M_{j}, N_{j}, M_{j}^{\prime}, N_{j}^{\prime}, j=1,2,3$ are sufficiently large integers. The last inequality holds since $|n|,\left|n^{\prime}\right| \geq 5$ and $i \geq j, i^{\prime} \geq j^{\prime}$, from which we can get a decay for $i, i^{\prime}$ and consequently for $j, j^{\prime}$ as well. Similar to (6.8), there exist large integers $L, L^{\prime}$

and also

$$
\begin{aligned}
(|n|-2)^{-\frac{M_{3}}{6}}(1+|n-m|)^{-N_{0}} & \lesssim(1+|m|)^{-L}, \\
\left(\left|n^{\prime}\right|-2\right)^{-\frac{M_{3}^{\prime}}{6}}\left(1+\left|n^{\prime}-m^{\prime}\right|\right)^{-N_{0}^{\prime}} & \lesssim\left(1+\left|m^{\prime}\right|\right)^{-L^{\prime}},
\end{aligned}
$$

$$
\begin{aligned}
(|n|-2)^{-\frac{M}{6}} \tilde{\chi}_{I_{n}} \lesssim \tilde{\chi}_{I^{0}} & \text { and } & & \left(\left|n^{\prime}\right|-2\right)^{-\frac{M^{\prime}}{6}} \tilde{\chi}_{I_{n^{\prime}}} \lesssim \tilde{\chi}_{I^{0}}, \\
(1+|m|)^{-\frac{L}{3}} \tilde{\chi}_{J_{m}} \lesssim \tilde{\chi}_{I^{0}} & \text { and } & & \left(1+\left|m^{\prime}\right|\right)^{-\frac{L^{\prime}}{3}} \tilde{\chi}_{J_{m^{\prime}}} \lesssim \tilde{\chi}_{I^{0}} .
\end{aligned}
$$

Then (6.10) can be estimated by

$$
\begin{aligned}
\lesssim & \sum_{|n|,\left|n^{\prime}\right| \geq 5} \sum_{m, m^{\prime} \in \mathbb{Z}}\left(\left(|n-2|^{-\frac{M_{3}}{3}}\right)\left(\left|n^{\prime}-2\right|^{-\frac{M_{3}^{\prime}}{3}}\right)(1+|m|)^{-L}\left(1+\left|m^{\prime}\right|\right)^{-L^{\prime}}\right. \\
& \left.\cdot\left\|f \chi_{I_{n}} \otimes \chi_{I_{n^{\prime}}} \mid\right\|_{p_{1}}\left\|g \chi_{J_{m}} \otimes \chi_{J_{m^{\prime}}}\right\|_{p_{2}}\left\|h \chi_{J_{m}} \otimes \chi_{J_{m^{\prime}}}\right\|_{p_{3}}\right)^{r} \\
\lesssim & \sum_{\substack{|n|,\left|n^{\prime}\right| \geq 5\\
}} \sum_{m, m^{\prime} \in \mathbb{Z}}\left(|n-2|^{-\frac{M_{3}}{6}}\left|n^{\prime}-2\right|^{-\frac{M_{3}^{\prime}}{6}}(1+|m|)^{-\frac{L}{3}}\left(1+\left|m^{\prime}\right|\right)^{-\frac{L^{\prime}}{3}}\right. \\
\lesssim & \left(\left\|f \tilde{\chi}_{R_{00}}\right\|_{p_{1}}\left\|g \tilde{\chi}_{R_{00}}\right\|_{p_{1}}\left\|g \tilde{\chi}_{R_{00}}\right\| h\left\|_{p_{2}}\right\| h \tilde{\chi}_{R_{00}}\left\|_{p_{00}}\right\|_{p_{3}}\right)^{r},
\end{aligned}
$$

Now we have proved the desired estimate for $T_{1,1}^{E_{2}, E_{2}^{\prime}, 0}(f, g, h)(x)$.

For $T_{1,1}^{E_{1}, E_{2}^{\prime}, 0}(f, g, h)(x)$, we just need to combine the ideas for $T_{1,1}^{E_{1}, E_{1}^{\prime}, 0}(f, g, h)(x)$ and $T_{1,1}^{E_{2}, E_{2}^{\prime}, 0}(f, g, h)(x)$ together. More precisely, since we have $I \subseteq 5 I^{0}$ and $I^{\prime} \subseteq$ $\left(5 I^{0}\right)^{c}$, we can do the decomposition

$$
f(x)=\sum_{n_{1} \in \mathbb{Z}} f(x) \cdot \chi_{I_{n_{1}}}\left(x_{1}\right), \quad g(x)=\sum_{n_{2} \in \mathbb{Z}} g(x) \cdot \chi_{I_{n_{2}}}\left(x_{1}\right), \quad h(x)=\sum_{n_{3} \in \mathbb{Z}} h(x) \cdot \chi_{I_{n_{3}}}\left(x_{1}\right) .
$$

As before, first consider $\left|n_{1}\right| \geq 10$.

$$
\begin{aligned}
& \left\|T_{1,1}^{E_{1}, E_{2}^{\prime}, 0}\left(f \chi_{I_{n_{1}}}, g \chi_{I_{n_{2}}}, h \chi_{I_{n_{3}}}\right)(x)\right\|_{r}^{r} \\
& \lesssim \sum_{\substack{\left|n^{\prime}\right| \geq 5 \\
m, m^{\prime} \in \mathbb{Z}}} \sum_{\substack{i, i^{\prime} \geq 0 \\
j, j^{\prime} \geq 0}} \sum_{\substack{I \subseteq 5 I^{0} \\
|I|=2^{-i}}} \sum_{I^{\prime} \subseteq I_{n^{\prime}}} \sum_{\substack{J \subseteq I^{\prime}\left|=2^{-i^{\prime}} \\
J^{\prime} \subseteq J_{m},\right| J\left|=J^{\prime}\right|=2^{-j}}}\left(\frac{1}{|I|\left|I^{\prime}\right|}\left(1+\frac{\operatorname{dist}\left(I_{n_{1}}, I\right)}{|I|}\right)^{-M_{1}}\right. \\
& \left(1+\frac{\operatorname{dist}\left(I_{n_{2}}, J\right)}{|J|}\right)^{-N_{1}}\left(1+\frac{\operatorname{dist}\left(I_{n_{3}}, J\right)}{|J|}\right)^{-N_{2}}\left(1+\frac{\operatorname{dist}\left(I^{\prime}, I^{0}\right)}{\left|I^{\prime}\right|}\right)^{-M_{3}^{\prime}}
\end{aligned}
$$




$$
\begin{aligned}
& \int_{\mathbb{R}^{2}}\left(1+\frac{\operatorname{dist}\left(x_{1}, I\right)}{|I|}\right)^{-M_{2}}\left(1+\frac{\operatorname{dist}\left(x_{2}, I^{\prime}\right)}{\left|I^{\prime}\right|}\right)^{-M_{2}^{\prime}} \\
& \cdot\left(1+\frac{\operatorname{dist}\left(x_{1}, J\right)}{|J|}\right)^{-N_{3}}\left(1+\frac{\operatorname{dist}\left(x_{2}, J^{\prime}\right)}{\left|J^{\prime}\right|}\right)^{-N_{3}^{\prime}} d x \\
& \left.\cdot\left\|f \chi_{I_{n_{1}}} \otimes \chi_{I_{n^{\prime}}}\right\|_{p_{1}}\left\|g \chi_{I_{n_{2}}} \otimes \chi_{I_{n^{\prime}}}\right\|_{p_{2}}\left\|h \chi_{I_{n_{3}}} \otimes \chi_{I_{n^{\prime}}}\right\|_{p_{3}}\right)^{r} \\
& \lesssim \sum_{\substack{\left|n^{\prime}\right| \geq 5 \\
m, m^{\prime} \in \mathbb{Z}}} \sum_{\substack{i, i^{\prime} \geq 0 \\
j, j^{\prime} \geq 0}} \sum_{\substack{I \subseteq 5 I^{0} \\
|I|=2^{-i}}} \sum_{\substack{I^{\prime} \subseteq I_{n^{\prime}} \\
\left|I^{\prime}\right|=2^{-i^{\prime}}}} \sum_{\substack{J \subseteq J_{m},|J|=J^{\prime} \subseteq J_{m^{\prime}},\left|J^{\prime}\right|=2^{-j^{\prime}} \\
J^{\prime}}}\left(2^{i+i^{\prime}}\left(1+2^{i}\left(\left|n_{1}\right|-6\right)\right)^{-M_{1}}\right. \\
& \cdot\left(1+2^{i^{\prime}}\left(\left|n^{\prime}\right|-2\right)\right)^{-M_{3}^{\prime}}\left(1+2^{j}\left|m-n_{2}\right|\right)^{-N_{1}} \\
& \cdot\left(1+2^{j}\left|m-n_{3}\right|\right)^{-N_{2}}(1+|m|)^{-N_{0}}\left(1+\left|m^{\prime}-n^{\prime}\right|\right)^{-N_{0}^{\prime}} \\
& \left.\cdot\left\|f \chi_{I_{n_{1}}} \otimes \chi_{I_{n^{\prime}}}\right\|_{p_{1}}\left\|g \chi_{I_{n_{2}}} \otimes \chi_{I_{m^{\prime}}}\right\|_{p_{2}}\left\|h \chi_{I_{n_{3}}} \otimes \chi_{I_{m^{\prime}}}\right\|_{p_{3}}\right)^{r} \\
& \lesssim \sum_{\substack{\left|n^{\prime}\right| \geq 5 \\
m, m^{\prime} \in \mathbb{Z}}}\left(\left(\left|n_{1}\right|-6\right)^{-\frac{M_{1}}{2}}\left(\left|n^{\prime}\right|-2\right)^{-\frac{M_{3}^{\prime}}{2}}\left(1+\left|n_{2}\right|\right)^{-L}\left(1+\left|n_{3}\right|\right)^{-L}\left(1+\left|n_{3}^{\prime}\right|\right)^{-L}\right. \\
& \cdot(1+|m|)^{-\frac{N_{0}}{2}}\left(1+\left|n^{\prime}-m^{\prime}\right|\right)^{-N_{0}^{\prime}} \\
& \left.\cdot\left\|f \chi_{I_{n_{1}}} \otimes \chi_{I_{n^{\prime}}}\right\|_{p_{1}}\left\|g \chi_{I_{n_{2}}} \otimes \chi_{I_{m^{\prime}}}\right\|_{p_{2}}\left\|h \chi_{I_{n_{3}}} \otimes \chi_{I_{m^{\prime}}}\right\|_{p_{3}}\right)^{r} \\
& \lesssim \sum_{\left|n^{\prime}\right| \geq 5} \sum_{m^{\prime} \in \mathbb{Z}}\left(\left(\left|n_{1}\right|-6\right)^{-\frac{M_{1}}{4}}\left(\left|n^{\prime}\right|-2\right)^{-\frac{M_{1}^{\prime}}{6}}\left(1+\left|n_{2}\right|\right)^{-\frac{L}{2}}\right. \\
& \left.\cdot\left(1+\left|n_{3}\right|\right)^{-\frac{L}{2}} \cdot\left(1+\left|m^{\prime}\right|\right)^{-\frac{L}{3}}\left\|f \tilde{\chi}_{R_{00}}\right\|_{p_{1}}\left\|g \tilde{\chi}_{R_{00}}\right\|_{p_{2}}\left\|h \tilde{\chi}_{R_{00}}\right\|_{p_{3}}\right)^{r} \\
& \lesssim\left(\left(\left|n_{1}\right|-6\right)^{-\frac{M_{1}}{4}}\left(1+\left|n_{2}\right|\right)^{-\frac{L}{2}}\left(1+\left|n_{3}\right|\right)^{-\frac{L}{2}}\left\|f \tilde{\chi}_{R_{00}}\right\|_{p_{1}}\left\|g \tilde{\chi}_{R_{00}}\right\|_{p_{2}}\left\|h \tilde{\chi}_{R_{00}}\right\|_{p_{3}}\right)^{r} \text {, }
\end{aligned}
$$

Thus,

$$
\begin{aligned}
& \left\|\sum_{\left|n_{1}\right|>10} \sum_{n_{2}, n_{3} \in \mathbb{Z}} T_{1,1}^{E_{1}, E_{2}^{\prime}, 0}\left(f \chi_{I_{n_{1}}}, g \chi_{I_{n_{2}}}, h \chi_{I_{n_{3}}}\right)(x)\right\|_{r}^{r} \\
\lesssim & \sum_{\left|n_{1}\right|>10} \sum_{n_{2}, n_{3} \in \mathbb{Z}}\left(\left(\left|n_{1}\right|-6\right)^{-\frac{M_{1}}{4}}\left(1+\left|n_{2}\right|\right)^{-\frac{L}{2}}\left(1+\left|n_{3}\right|\right)^{-\frac{L}{2}}\right. \\
& \left.\cdot\left\|f \tilde{\chi}_{R_{00}}\right\|_{p_{1}}\left\|g \tilde{\chi}_{R_{00}}\right\|_{p_{2}}\left\|h \tilde{\chi}_{R_{00}}\right\|_{p_{3}}\right)^{r} \\
\lesssim & \left(\left\|f \tilde{\chi}_{R_{00}}\right\|_{p_{1}}\left\|g \tilde{\chi}_{R_{00}}\right\|_{p_{2}}\left\|h \tilde{\chi}_{R_{00}}\right\|_{p_{3}}\right)^{r} .
\end{aligned}
$$

When $\left|n_{1}\right|<10$, as before we can get some decay factors by using ([6.8), and with Theorem 1.7 one can get

$$
\left\|\sum_{\substack{\left|n_{1}\right|<10 \\ n_{2}, n_{3} \in \mathbb{Z}}} T_{1,1}^{E_{1}, E_{2}^{\prime}, 0}\left(f \chi_{I_{n_{1}}}, g \chi_{I_{n_{2}}}, h \chi_{I_{n_{3}}}\right)(x)\right\|_{r}^{r}
$$




$$
\begin{aligned}
& \lesssim \sum_{\left|n^{\prime}\right| \geq 5} \sum_{m^{\prime} \in \mathbb{Z}} \sum_{\substack{\left|n_{1}\right|<10 \\
n_{2}, n_{3} \in \mathbb{Z}}}\left(\left(1+\left|n_{2}\right|\right)^{-M^{\prime}}\left(1+\left|n_{3}\right|\right)^{-M^{\prime}}\left(\left|n^{\prime}\right|-2\right)^{-M^{\prime}}\left(1+\left|m^{\prime}\right|\right)^{-M^{\prime}}\right. \\
&\left.\left\|T_{1,1}^{E_{1}, E_{2}^{\prime}, 0}\left(f \cdot \chi_{I_{n_{1}}} \otimes \chi_{I_{n^{\prime}}}, g \cdot \chi_{I_{n_{2}}} \otimes \chi_{I_{m^{\prime}}}, h \cdot \chi_{I_{n_{3}}} \otimes \chi_{I_{m^{\prime}}}\right)(x)\right\|_{r}\right)^{r} \\
& \sum_{\left|n^{\prime}\right| \geq 5} \sum_{\substack{\left|n_{1}\right|<10 \\
n_{2}, n_{3} \in \mathbb{Z}}}\left(\left(1+\left|n_{2}\right|\right)^{-M^{\prime}}\left(1+\left|n_{3}\right|\right)^{-M^{\prime}}\left(\left|n^{\prime}\right|-2\right)^{-M^{\prime}}\right. \\
&\left.\cdot\left\|f \cdot \chi_{I_{n_{1}}} \otimes \chi_{I_{n^{\prime}}}\right\|_{p_{1}}\left\|g \cdot \chi_{I_{n_{2}}} \otimes \chi_{I_{n^{\prime}}}\right\|_{p_{2}}\left\|h \cdot \chi_{I_{n_{3}}} \otimes \chi_{I_{n^{\prime}}}\right\|_{p_{3}}\right)^{r} \\
& \lesssim \quad\left(\left\|f \tilde{\chi}_{R_{00}}\right\|_{p_{1}}\left\|g \tilde{\chi}_{R_{00}}\right\|_{p_{2}}\left\|h \tilde{\chi}_{R_{00}}\right\|_{p_{3}}\right)^{r} .
\end{aligned}
$$

for some sufficiently large integer $M^{\prime}$.

We omit the remaining details, and we are done with $T_{1,1}^{E, E^{\prime}, 0}(f, g, h)(x)$.

Then we turn to the study of $T_{1, m_{M, k_{0}}}^{E, E^{\prime}, 0}(f, g, h)(x)$. From the condition that $m_{M, k_{0}}$ satisfies, we see $T_{1, m_{M, k_{0}}}^{E, E^{\prime}, 0}(f, g, h)(x)$ corresponds to a classical trilinear paraproduct (see [7, 20]) in the second parameter, while in the first parameter the form is like what happens for $T_{1,1}^{E, E^{\prime}, 0}(f, g, h)(x)$. We have

$$
\begin{aligned}
\left\|T_{1, m_{M, k_{0}}}^{E, E^{\prime}, 0}(f, g, h)(x)\right\|_{r}^{r} \lesssim & \| \sum_{I \in \mathcal{I}, I^{\prime} \in \mathcal{I}^{\prime}} \sum_{\substack{J \in \mathcal{J} \\
\left|\omega_{J}^{3}\right| \leq\left|\omega_{I}^{2}\right|}} \frac{1}{|I|^{\frac{1}{2}}} \frac{1}{|J|^{\frac{1}{2}}} \frac{1}{\left|I^{\prime}\right|}\left\langle f, \phi_{I}^{1} \otimes \phi_{I^{\prime}}^{1}\right\rangle\left\langle g, \phi_{J}^{1} \otimes \phi_{I^{\prime}}^{2}\right\rangle \\
& \cdot\left\langle h, \phi_{J}^{2} \otimes \phi_{I^{\prime}}^{3}\right\rangle\left\langle\phi_{I}^{2}, \phi_{J}^{3}\right\rangle \phi_{I}^{3}\left(x_{1}\right) \phi_{I^{\prime}}^{4}\left(x_{2}\right) \varphi_{0}\left(x_{1}\right) \varphi_{0}^{\prime}\left(x_{2}\right) \|_{r},
\end{aligned}
$$

where the families $\left(\phi_{I}^{j}\right)_{I \in \mathcal{I}},\left(\phi_{J}^{j}\right)_{I \in \mathcal{J}}$ are defined as $\left(\phi_{J}^{j}\right)_{J \in \mathcal{J}}$ in Definition 5 , and two of $\left(\phi_{I^{\prime}}^{l}\right)_{I^{\prime} \in \mathcal{I}^{\prime}}(l=1,2,3,4)$ are lacunary functions. Actually this is an easier case than $T_{1,1}^{E, E^{\prime}, 0}(f, g, h)(x)$, since the implicit symbol in the second parameter satisfies a stronger condition than the one in $T_{1,1}^{E, E^{\prime}, 0}(f, g, h)(x)$ and there is only one class of dyadic intervals on the second parameter in the above paraproducts. That means one can mimic the proof for $T_{1,1}^{E, E^{\prime}, 0}(f, g, h)(x)$ to get the desired estimate, where the following theorem is needed, which plays a similar role as Theorem 1.7 for $T_{1,1}^{E, E^{\prime}, 0}(f, g, h)(x)$.

Theorem 6.2. For $f, g, h \in \mathcal{S}\left(\mathbb{R}^{2}\right)$, the bi-parameter operators

$$
T_{m^{\prime}}(f, g, h)(x):=\int_{\mathbb{R}^{6}} m^{\prime}(\xi, \eta, \zeta) \hat{f}(\xi) \hat{g}(\eta) \hat{h}(\zeta) e^{2 \pi i(\xi+\eta+\zeta) \cdot x} d \xi d \eta d \zeta
$$

map $L^{p_{1}} \times L^{p_{2}} \times L^{p_{3}} \rightarrow L^{r}$ for $1<p_{1}, p_{2}, p_{3}<\infty$ with $1 / p_{1}+1 / p_{2}+1 / p_{3}=1 / r$ and $0<r<\infty$, as long as the smooth symbol $m^{\prime}$ satisfies

$$
\left|\partial_{\xi_{1}}^{\alpha_{1}} \partial_{\xi_{2}}^{\alpha_{2}} \partial_{\eta_{1}}^{\beta_{1}} \partial_{\eta_{2}}^{\beta_{2}} \partial_{\zeta_{1}}^{\gamma_{1}} \partial_{\zeta_{2}}^{\gamma_{2}} m^{\prime}(\xi, \eta, \zeta)\right|
$$




$$
\begin{gathered}
\lesssim \sum_{\substack{\beta_{1}^{\prime}+\beta_{1}^{\prime \prime}=\beta_{1} \\
\gamma_{1}^{\prime}+\gamma_{1}^{\prime \prime}=\gamma_{1}}}\left(1+\left|\xi_{1}\right|+\left|\eta_{1}\right|+\left|\zeta_{1}\right|\right)^{-\left(\alpha_{1}+\beta_{1}^{\prime}+\gamma_{1}^{\prime}\right)}\left(1+\left|\eta_{1}\right|+\left|\zeta_{1}\right|\right)^{-\left(\beta_{1}^{\prime \prime}+\gamma_{1}^{\prime \prime}\right)} \\
\cdot\left(1+\left|\xi_{2}\right|+\left|\eta_{2}\right|+\left|\zeta_{2}\right|\right)^{-\left(\alpha_{2}+\beta_{2}+\gamma_{2}\right)}
\end{gathered}
$$

for sufficiently many multi-indices $\alpha_{1}, \beta_{1}, \gamma_{1}$ and $\alpha_{2}, \beta_{2}, \gamma_{2}$.

Proof. This is essentially a corollary of Theorem 1.7. since the symbol $\mathrm{m}^{\prime}$ satisfies a stronger condition in the second parameter than $m_{1}(\xi, \eta, \zeta) m_{2}(\eta, \zeta)$. To get the result, one just needs to keep the argument in [3] on the second parameter, and do the necessary modification as in Theorem 1.7 on the first parameter. We omit the details here.

Now we are ready to prove the estimate for $T_{1, m_{M, k_{0}}}^{E, E^{\prime}, 0}(f, g, h)(x)$.

Taking advantage of the fact that $|I|,\left|I^{\prime}\right| \leq 1$, we can split

$$
\begin{aligned}
& T_{1, m_{M, k_{0}}}^{E, E^{\prime}, 0}(f, g, h)=\left\{\left(\sum_{\substack{I \subseteq 5 I^{0} \\
I^{\prime} \subseteq 5 I^{0}}}+\sum_{\substack{I \subseteq\left(5 I^{0}\right)^{c} \\
I^{\prime} \subseteq 5 I^{0}}}+\sum_{\substack{I \subseteq 5 I^{0} \\
I^{\prime} \subseteq\left(5 I^{0}\right)^{c}}}+\sum_{\substack{I \subseteq\left(5 I^{0}\right)^{c} \\
I^{\prime} \subseteq\left(5 I^{0}\right)^{c}}}\right) \sum_{\substack{J \in \mathcal{J} \\
\left|\omega_{J}^{3}\right| \leq\left|\omega_{I}^{2}\right|}} \frac{1}{|I|^{\frac{1}{2}}} \frac{1}{|J|^{\frac{1}{2}}} \frac{1}{\left|I^{\prime}\right|}\right. \\
& \left\langle f, \phi_{I}^{1} \otimes \phi_{I^{\prime}}^{1}\right\rangle\left\langle g, \phi_{J}^{1} \otimes \phi_{I^{\prime}}^{2}\right\rangle\left\langle h, \phi_{J}^{2} \otimes \phi_{I^{\prime}}^{3}\right\rangle\left\langle\phi_{I}^{2}, \phi_{J}^{3}\right\rangle \phi_{I}^{3}\left(x_{1}\right) \phi_{I^{\prime}}^{4}\left(x_{2}\right) \varphi_{0}\left(x_{1}\right) \varphi_{0}^{\prime}\left(x_{2}\right) \\
= & T_{1, m_{M, k_{0}}}^{E_{1}, E_{1}^{\prime}, 0}(f, g, h)+T_{1, m_{M, k_{0}}}^{E_{2}, E_{1}^{\prime}, 0}(f, g, h)+T_{1, m_{M, k_{0}}}^{E_{1} E_{2}^{\prime}, 0}(f, g, h)+T_{1, m_{M, k_{0}}}^{E_{2} E_{2}^{\prime}, 0}(f, g, h) .
\end{aligned}
$$

We start with $T_{1, m_{M, k_{0}}}^{E_{1}, E_{1}^{\prime}, 0}(f, g, h)$. We still consider the decomposition (6.6), and we can write

$$
\begin{gathered}
T_{1, m_{M, k_{0}}}^{E_{1}, E_{1}^{\prime}, 0}(f, g, h)(x)= \\
\sum_{n_{1}, n_{1}^{\prime}} \sum_{n_{2}, n_{2}^{\prime}} \sum_{n_{3}, n_{3}^{\prime}} T_{1, m_{M, k_{0}}}^{E_{1}, E_{1}^{\prime}, 0}\left(f \cdot \chi_{I_{n_{1}}} \otimes \chi_{I_{n_{1}^{\prime}}}, g \cdot \chi_{I_{n_{2}}} \otimes \chi_{I_{n_{2}^{\prime}}}, h \cdot \chi_{I_{n_{3}}} \otimes \chi_{I_{n_{3}^{\prime}}}\right)(x) .
\end{gathered}
$$

When $\left|n_{1}\right|,\left|n_{1}^{\prime}\right|>10$, we write

$$
\begin{aligned}
& \left\|T_{1, m_{M, k_{0}}}^{E_{1}, E_{1}^{\prime}, 0}\left(f \cdot \chi_{I_{n_{1}}} \otimes \chi_{I_{n_{1}^{\prime}}}, g \cdot \chi_{I_{n_{2}}} \otimes \chi_{I_{n_{2}^{\prime}}}, h \cdot \chi_{I_{n_{3}}} \otimes \chi_{I_{n_{3}^{\prime}}}\right)(x)\right\|_{r} \\
= & \| \sum_{I \in \mathcal{I}, I^{\prime} \in \mathcal{I}^{\prime}} \sum_{\substack{J \in \mathcal{J} \\
\left|\omega_{J}^{3}\right| \leq\left|\omega_{I}^{2}\right|}} \frac{1}{|I|^{\frac{1}{2}}} \frac{1}{|J|^{\frac{1}{2}}} \frac{1}{\left|I^{\prime}\right|}\left\langle f \chi_{I_{n_{1}}} \otimes \chi_{I_{n_{1}^{\prime}}}, \phi_{I}^{1} \otimes \phi_{I^{\prime}}^{1}\right\rangle\left\langle g \chi_{I_{n_{2}}} \otimes \chi_{I_{n_{2}^{\prime}}}, \phi_{J}^{1} \otimes \phi_{I^{\prime}}^{2}\right\rangle \\
& \cdot\left\langle h \chi_{I_{n_{3}}} \otimes \chi_{I_{n_{3}^{\prime}}}, \phi_{J}^{2} \otimes \phi_{I^{\prime}}^{3}\right\rangle\left\langle\phi_{I}^{2}, \phi_{J}^{3}\right\rangle \phi_{I}^{3}\left(x_{1}\right) \phi_{I^{\prime}}^{4}\left(x_{2}\right) \varphi_{0}\left(x_{1}\right) \varphi_{0}^{\prime}\left(x_{2}\right) \|_{r} .
\end{aligned}
$$

Then we use Hölder's inequality to get

$$
\begin{aligned}
& \| \frac{1}{|I|^{\frac{1}{2}}} \frac{1}{|J|^{\frac{1}{2}}} \frac{1}{\left|I^{\prime}\right|}\left\langle f \chi_{I_{n_{1}}} \otimes \chi_{I_{n_{1}^{\prime}}}, \phi_{I}^{1} \otimes \phi_{I^{\prime}}^{1}\right\rangle\left\langle g \chi_{I_{n_{2}}} \otimes \chi_{I_{n_{2}^{\prime}}}, \phi_{J}^{1} \otimes \phi_{I^{\prime}}^{2}\right\rangle \\
& \quad \cdot\left\langle h \chi_{I_{n_{3}}} \otimes \chi_{I_{n_{3}^{\prime}}}, \phi_{J}^{2} \otimes \phi_{I^{\prime}}^{3}\right\rangle\left\langle\phi_{I}^{2}, \phi_{J}^{3}\right\rangle \phi_{I}^{3}\left(x_{1}\right) \phi_{I^{\prime}}^{4}\left(x_{2}\right) \varphi_{0}\left(x_{1}\right) \varphi_{0}^{\prime}\left(x_{2}\right) \|_{r}
\end{aligned}
$$




$$
\begin{aligned}
& \lesssim \frac{1}{|I|^{2}} \frac{1}{|J|^{2}} \frac{1}{\left|I^{\prime}\right|^{3}} \\
& \cdot\left(1+\frac{\operatorname{dist}\left(I_{n_{1}}, I\right)}{|I|}\right)^{-M_{1}}\left(1+\frac{\operatorname{dist}\left(I_{n_{1}^{\prime}}, I^{\prime}\right)}{\left|I^{\prime}\right|}\right)^{-M_{1}^{\prime}}\left(\left\|f \chi_{I_{n_{1}}} \otimes \chi_{I_{n_{1}^{\prime}}}\right\|_{p_{1}}\left(|I|\left|I^{\prime}\right|\right)^{\frac{p_{1}-1}{p_{1}}}\right) \\
& \cdot\left(1+\frac{\operatorname{dist}\left(I_{n_{2}}, J\right)}{|J|}\right)^{-N_{1}}\left(1+\frac{\operatorname{dist}\left(I_{n_{2}^{\prime}}, I^{\prime}\right)}{\left|I^{\prime}\right|}\right)^{-M_{2}^{\prime}}\left(\left\|g \chi_{I_{n_{2}}} \otimes \chi_{I_{n_{2}}^{\prime}}\right\|_{p_{2}}\left(|J||I|^{\prime}\right)^{\frac{p_{2}-1}{p_{2}}}\right) \\
& \left(1+\frac{\operatorname{dist}\left(I_{n_{3}}, J\right)}{|J|}\right)^{-N_{2}}\left(1+\frac{\operatorname{dist}\left(I_{n_{3}^{\prime}}, I^{\prime}\right)}{\left|I^{\prime}\right|}\right)^{-M_{3}^{\prime}}\left(\left\|h \chi_{I_{n_{3}}} \otimes \chi_{I_{n_{3}^{\prime}}}\right\|_{p_{3}}\left(|J|\left|I^{\prime}\right|\right)^{\frac{p_{3}-1}{p_{3}}}\right) \\
& \cdot\left(|I|\left|I^{\prime}\right|\right)^{\frac{1}{r}} \int_{\mathbb{R}^{2}}\left(1+\frac{\operatorname{dist}\left(x_{1}, I\right)}{|I|}\right)^{-M_{2}}\left(1+\frac{\operatorname{dist}\left(x_{1}, J\right)}{|J|}\right)^{-N_{3}} d x \\
& \lesssim \frac{1}{|I|}\left(\frac{|I|}{|J|}\right)^{\frac{1}{p_{2}}+\frac{1}{p_{3}}}\left(1+\frac{\operatorname{dist}\left(I_{n_{1}}, I\right)}{|I|}\right)^{-M_{1}}\left(1+\frac{\operatorname{dist}\left(I_{n_{1}^{\prime}}, I^{\prime}\right)}{\left|I^{\prime}\right|}\right)^{-M_{1}^{\prime}}\left(1+\frac{\operatorname{dist}\left(I_{n_{2}}, J\right)}{|J|}\right)^{-N_{1}} \\
& \cdot\left(1+\frac{\operatorname{dist}\left(I_{n_{2}^{\prime}}, I^{\prime}\right)}{\left|I^{\prime}\right|}\right)^{-M_{2}^{\prime}}\left(1+\frac{\operatorname{dist}\left(I_{n_{3}}, J\right)}{|J|}\right)^{-N_{2}}\left(1+\frac{\operatorname{dist}\left(I_{n_{3}^{\prime}}, I^{\prime}\right)}{\left|I^{\prime}\right|}\right)^{-M_{3}^{\prime}} \\
& \cdot \int_{\mathbb{R}}\left(1+\frac{\operatorname{dist}\left(x_{1}, I\right)}{|I|}\right)^{-M_{2}}\left(1+\frac{\operatorname{dist}\left(x_{1}, J\right)}{|J|}\right)^{-N_{3}} d x_{1} \\
& \cdot\left\|f \chi_{I_{n_{1}}} \otimes \chi_{I_{n_{1}^{\prime}}}\right\|_{p_{1}}\left\|g \chi_{I_{n_{2}}} \otimes \chi_{I_{n_{2}^{\prime}}}\right\|_{p_{2}}\left\|h \chi_{I_{n_{3}}} \otimes \chi_{I_{n_{3}^{\prime}}}\right\|_{p_{3}} \text {, }
\end{aligned}
$$

where $M_{j}, M_{j}^{\prime}, N_{j}$ are sufficiently large integers and $\phi_{I}^{j}, \phi_{J}^{j}, \phi_{I^{\prime}}^{k}$ are $L^{2}$-normalized bump functions adapted to $I, J, I^{\prime}$ for $j=1,2,3$ and $k=1,2,3,4$. Taking advantage of $\left|\omega_{J}^{3}\right| \leq\left|\omega_{I}^{2}\right|$ and $|I|,|J|,\left|I^{\prime}\right| \leq 1$, one can get

$$
\begin{aligned}
& \left\|T_{1, m_{M, k_{0}}}^{E_{1}, E_{1}^{\prime}, 0}\left(f \cdot \chi_{I_{n_{1}}} \otimes \chi_{I_{n_{1}^{\prime}}}, g \cdot \chi_{I_{n_{2}}} \otimes \chi_{I_{n_{2}^{\prime}}}, h \cdot \chi_{I_{n_{3}}} \otimes \chi_{I_{n_{3}^{\prime}}}\right)(x)\right\|_{r}^{r} \\
& \lesssim \sum_{\substack{i, i^{\prime} \geq 0 \\
j \geq 0}} \sum_{\substack{I, I^{\prime} \subseteq 5 I^{0} \\
|I|=I^{-i} \\
\left|I^{\prime}\right|=2^{-i^{\prime}}}} \sum_{m \in \mathbb{Z}} \sum_{\substack{J \subseteq J_{m} \\
|J|=2^{-j}}}\left(\frac{1}{|I|}\left(1+\frac{\operatorname{dist}\left(I_{n_{1}}, I\right)}{|I|}\right)^{-M_{1}}\left(1+\frac{\operatorname{dist}\left(I_{n_{1}^{\prime}}, I^{\prime}\right)}{\left|I^{\prime}\right|}\right)^{-M_{1}^{\prime}}\right. \\
& \cdot\left(1+\frac{\operatorname{dist}\left(I_{n_{2}}, J\right)}{|J|}\right)^{-N_{1}}\left(1+\frac{\operatorname{dist}\left(I_{n_{2}^{\prime}}, I^{\prime}\right)}{\left|I^{\prime}\right|}\right)^{-M_{2}^{\prime}}\left(1+\frac{\operatorname{dist}\left(I_{n_{3}}, J\right)}{|J|}\right)^{-N_{2}} \\
& \cdot\left(1+\frac{\operatorname{dist}\left(I_{n_{3}^{\prime}}, I^{\prime}\right)}{\left|I^{\prime}\right|}\right)^{-M_{3}^{\prime}} \int_{\mathbb{R}}\left(1+\frac{\operatorname{dist}\left(x_{1}, I\right)}{|I|}\right)^{-M_{2}}\left(1+\frac{\operatorname{dist}\left(x_{1}, J\right)}{|J|}\right)^{-N_{3}} d x_{1} \\
& \left.\cdot\left\|f \chi_{I_{n_{1}}} \otimes \chi_{I_{n_{1}^{\prime}}}\right\|_{p_{1}}\left\|g \chi_{I_{n_{2}}} \otimes \chi_{I_{n_{2}^{\prime}}}\right\|_{p_{2}}\left\|h \chi_{I_{n_{3}}} \otimes \chi_{I_{n_{3}^{\prime}}}\right\|_{p_{3}}\right)^{r} \\
& \lesssim \sum_{\substack{i, i^{\prime} \geq 0 \\
j \geq 0}} \sum_{\substack{I, I^{\prime} \subseteq 5 I_{0} \\
|I|=I^{-i} \\
\left|I^{\prime}\right|=2^{-i^{\prime}}}} \sum_{m \in \mathbb{Z}} \sum_{\substack{J \subseteq J_{m} \\
|J|=2^{-j}}}\left(2^{i}\left(1+2^{i}\left(\left|n_{1}\right|-6\right)\right)^{-M_{1}}\left(1+2^{i^{\prime}}\left(\left|n_{1}^{\prime}\right|-6\right)\right)^{-M_{1}^{\prime}}\right. \\
& \cdot\left(1+2^{j}\left|m-n_{2}\right|\right)^{-N_{1}}\left(1+2^{j}\left|m-n_{3}\right|\right)^{-N_{2}}\left(1+2^{i^{\prime}}\left|n_{2}^{\prime}\right|\right)^{-M_{2}^{\prime}}\left(1+2^{i^{\prime}}\left|n_{3}^{\prime}\right|\right)^{-M_{3}^{\prime}}
\end{aligned}
$$




$$
\begin{aligned}
& \left.\cdot(1+|m|)^{-N_{0}}\left\|f \chi_{I_{n_{1}}} \otimes \chi_{I_{n_{1}^{\prime}}}\right\|_{p_{1}}\left\|g \chi_{I_{n_{2}}} \otimes \chi_{I_{n_{2}^{\prime}}}\right\|_{p_{2}}\left\|\chi_{I_{n_{3}}} \otimes \chi_{I_{n_{3}^{\prime}}}\right\|_{p_{3}}\right)^{r} \\
\lesssim & \sum_{m \in \mathbb{Z}}\left(\left(\left|n_{1}\right|-6\right)^{-\frac{M_{1}}{2}}\left(\left|n_{1}^{\prime}\right|-6\right)^{-\frac{M_{1}^{\prime}}{2}}\left(1+\left|n_{2}\right|\right)^{-L}\left(1+\left|n_{3}\right|\right)^{-L}\right. \\
& \cdot\left(1+\left|n_{2}^{\prime}\right|\right)^{-M_{2}^{\prime}}\left(1+\left|n_{3}^{\prime}\right|\right)^{-M_{3}^{\prime}}(1+|m|)^{-\frac{N_{0}}{2}} \\
& \left.\cdot\left\|f \chi_{I_{n_{1}}} \otimes \chi_{I_{n_{1}^{\prime}}}\right\|_{p_{1}}\left\|g \chi_{I_{n_{2}}} \otimes \chi_{I_{n_{2}^{\prime}}}\right\|_{p_{2}}\left\|h \chi_{I_{n_{3}}} \otimes \chi_{I_{n_{3}^{\prime}}}\right\|_{p_{3}}\right)^{r} \\
\lesssim & \left(\left(1+\left|n_{1}\right|\right)^{-\frac{M_{1}}{2}}\left|n_{1}^{\prime}\right|^{-\frac{M_{1}^{\prime}}{2}}\left(1+\left|n_{2}\right|\right)^{-L}\left(1+\left|n_{3}\right|\right)^{-L}\left(1+\left|n_{2}^{\prime}\right|\right)^{-M_{2}^{\prime}}\left(1+\left|n_{3}^{\prime}\right|\right)^{-M_{3}^{\prime}}\right. \\
& \left.\cdot\left\|f \chi_{I_{n_{1}}} \otimes \chi_{I_{n_{1}^{\prime}}}\right\|_{p_{1}}\left\|g \chi_{I_{n_{2}}} \otimes \chi_{I_{n_{2}^{\prime}}}\right\|_{p_{2}}\left\|h \chi_{I_{n_{3}}} \otimes \chi_{I_{n_{3}^{\prime}}}\right\|_{p_{3}}\right)^{r} \\
\lesssim & \left(\left(\left|n_{1}\right|-6\right)^{-\frac{M_{1}}{4}}\left(\left|n_{1}^{\prime}\right|-6\right)^{-\frac{M_{1}^{\prime}}{4}}\left(1+\left|n_{2}\right|\right)^{-\frac{L}{2}}\left(1+\left|n_{2}^{\prime}\right|\right)^{-\frac{M_{2}^{\prime}}{2}}\right. \\
& \left.\cdot\left(1+\left|n_{3}\right|\right)^{-\frac{L}{2}}\left(1+\left|n_{3}^{\prime}\right|\right)^{-\frac{M_{3}^{\prime}}{2}}\left\|f \tilde{\chi}_{R_{00}}\right\|_{p_{1}}\left\|g \tilde{\chi}_{R_{00}}\right\|_{p_{2}}\left\|h \tilde{\chi}_{R_{00}}\right\|_{p_{3}}\right)^{r}
\end{aligned}
$$

where the positive integers $N_{0}=\min \left\{M_{2}, N_{3}\right\}, L$ are sufficiently large, and the summation over $i^{\prime}$ is allowed since $\left|n_{1}^{\prime}\right| \geq 10$. Thus,

$$
\begin{aligned}
& \left\|\sum_{\substack{\left|n_{1}\right|>10 \\
\left|n_{1}^{\prime}\right|>10}} \sum_{n_{2}, n_{3}, n_{2}^{\prime}, n_{3}^{\prime} \in \mathbb{Z}} T_{1,1}^{E_{1}, E_{1}^{\prime}, 0}\left(f \cdot \chi_{I_{n_{1}}} \otimes \chi_{I_{n_{1}^{\prime}}}, g \cdot \chi_{I_{n_{2}}} \otimes \chi_{I_{n_{2}^{\prime}}}, h \cdot \chi_{I_{n_{3}}} \otimes \chi_{I_{n_{3}^{\prime}}}\right)(x)\right\|_{r}^{r} \\
\lesssim & \sum_{\left|n_{1}\right|>10,\left|n_{1}^{\prime}\right|>10} \sum_{n_{2}, n_{3}, n_{2}^{\prime}, n_{3}^{\prime} \in \mathbb{Z}}\left(\left(\left|n_{1}\right|-6\right)^{-\frac{M_{1}}{4}}\left(\left|n_{1}^{\prime}\right|-6\right)^{-\frac{M_{1}^{\prime}}{4}}\left(1+\left|n_{2}\right|\right)^{-\frac{L}{2}}\left(1+\left|n_{2}^{\prime}\right|\right)^{-\frac{M_{2}^{\prime}}{2}}\right. \\
& \left.\cdot\left(1+\left|n_{3}\right|\right)^{-\frac{L}{2}}\left(1+\left|n_{3}^{\prime}\right|\right)^{-\frac{M_{3}^{\prime}}{2}}\left\|f \tilde{\chi}_{R_{00}}\right\|_{p_{1}}\left\|g \tilde{\chi}_{R_{00}}\right\|_{p_{2}}\left\|h \tilde{\chi}_{R_{00}}\right\|_{p_{3}}\right)^{r} \\
\lesssim & \left(\left\|f \tilde{\chi}_{R_{00}}\right\|_{p_{1}}\left\|g \tilde{\chi}_{R_{00}}\right\|_{p_{2}}\left\|h \tilde{\chi}_{R_{00}}\right\|_{p_{3}}\right)^{r},
\end{aligned}
$$

As before when $\left|n_{1}\right| \leq 10$ or $\left|n_{1}^{\prime}\right| \leq 10$ things are different, since we cannot take the summation over $i$ or $i^{\prime}$. Without loss of generality, we assume both $\left|n_{1}\right| \leq 10$ and $\left|n_{1}^{\prime}\right| \leq 10$.

$$
\begin{aligned}
& \left\|\sum_{\substack{\left|n_{1}\right|,\left|n_{1}^{\prime}\right| \leq 10 \\
n_{2}, n_{2}^{\prime}, n_{3}, n_{3}^{\prime} \in \mathbb{Z}}} T_{1, m_{M, k_{0}}}^{E_{1}, E_{1}^{\prime}, 0}\left(f \cdot \chi_{I_{n_{1}}} \otimes \chi_{I_{n_{1}^{\prime}}}, g \cdot \chi_{I_{n_{2}}} \otimes \chi_{I_{n_{2}^{\prime}}}, h \cdot \chi_{I_{n_{3}}} \otimes \chi_{I_{n_{3}^{\prime}}}\right)(x)\right\|_{r} \\
\lesssim & \left\|f \tilde{\chi}_{R_{00}}\right\|_{p_{1}}\left\|g \tilde{\chi}_{R_{00}}\right\|_{p_{2}}\left\|h \tilde{\chi}_{R_{00}}\right\|_{p_{3}} .
\end{aligned}
$$

Recall that when $I, I^{\prime} \subseteq 5 I^{0}$ and $J \in J_{m}$, in (6.11) we can write

$$
\begin{gathered}
\left\langle\phi_{I}^{2}, \phi_{J}^{3}\right\rangle \approx(1+|m|)^{-L}\left\langle\phi_{I}^{2}, \tilde{\phi}_{J}^{3}\right\rangle, \\
\left\langle g \chi_{I_{n_{2}}} \otimes \chi_{I_{n_{2}^{\prime}}}, \phi_{J}^{1} \otimes \phi_{I^{\prime}}^{2}\right\rangle \approx\left(1+\left|n_{2}-m\right|\right)^{-M}\left(1+\left|n_{2}^{\prime}\right|\right)^{-M^{\prime}}\left\langle g \chi_{I_{n_{2}}} \otimes \chi_{I_{n_{2}^{\prime}}}, \tilde{\phi}_{J}^{1} \otimes \tilde{\phi}_{I^{\prime}}^{2}\right\rangle, \\
\left\langle h \chi_{I_{n_{3}}} \otimes \chi_{I_{n_{3}^{\prime}}}, \phi_{J}^{2} \otimes \phi_{I^{\prime}}^{3}\right\rangle \approx\left(1+\left|n_{3}-m\right|\right)^{-M}\left(1+\left|n_{3}^{\prime}\right|\right)^{-M^{\prime}}\left\langle h \chi_{I_{n_{3}}} \otimes \chi_{I_{n_{3}^{\prime}}}, \tilde{\phi}_{J}^{2} \otimes \tilde{\phi}_{I^{\prime}}^{3}\right\rangle, \\
\left(1+\left|n_{2}-m\right|\right)^{-M}(1+|m|)^{-L / 3} \approx\left(1+\left|n_{2}\right|\right)^{-M^{\prime}},
\end{gathered}
$$


where $M^{\prime}$ can be sufficiently large.

$$
\left(1+\left|n_{3}-m\right|\right)^{-M}(1+|m|)^{-L / 3} \approx\left(1+\left|n_{3}\right|\right)^{-M^{\prime}}
$$

That means we can use Theorem 6.2 with additional decay factors $\left(1+\left|n_{2}\right|\right)^{-M^{\prime}}(1+$ $\left.\left|n_{3}\right|\right)^{-M^{\prime}}\left(1+\left|n_{2}^{\prime}\right|\right)^{-M^{\prime}}\left(1+\left|n_{3}^{\prime}\right|\right)^{-M^{\prime}}$. Thus,

$$
\begin{aligned}
& \left\|\sum_{\substack{\left|n_{1}\right|,\left|n_{1}^{\prime}\right| \leq 10 \\
n_{2}, n_{2}^{\prime}, n_{3}, n_{3}^{\prime} \in \mathbb{Z}}} T_{1,1}^{E_{1}, E_{1}^{\prime}, 0}\left(f \cdot \chi_{I_{n_{1}}} \otimes \chi_{I_{n_{1}^{\prime}}}, g \cdot \chi_{I_{n_{2}}} \otimes \chi_{I_{n_{2}^{\prime}}}, h \cdot \chi_{I_{n_{3}}} \otimes \chi_{I_{n_{3}^{\prime}}}\right)(x)\right\|_{r} \\
\lesssim & \sum_{\substack{\left|n_{1}\right|,\left|n_{1}^{\prime}\right| \leq 10 \\
n_{2}, n_{2}^{\prime}, n_{3}, n_{3}^{\prime} \in \mathbb{Z}}}\left(1+\left|n_{2}\right|\right)^{-M^{\prime}}\left(1+\left|n_{2}^{\prime}\right|\right)^{-M^{\prime}} \cdot\left(1+\left|n_{3}\right|\right)^{-M^{\prime}}\left(1+\left|n_{3}^{\prime}\right|\right)^{-M^{\prime}} \\
& \cdot\left\|f \cdot \chi_{I_{n_{1}}} \otimes \chi_{I_{n_{1}^{\prime}}}\right\|_{p_{1}}\left\|g \cdot \chi_{I_{n_{2}}} \otimes \chi_{I_{n_{2}^{\prime}}}\right\|_{p_{2}}\left\|h \cdot \chi_{I_{n_{3}}} \otimes \chi_{I_{n_{3}^{\prime}}}\right\|_{p_{3}} \\
\lesssim & \left\|f \tilde{\chi}_{R_{00}}\right\|_{p_{1}}\left\|g \tilde{\chi}_{R_{00}}\right\|_{p_{2}}\left\|h \tilde{\chi}_{R_{00}}\right\|_{p_{3}} .
\end{aligned}
$$

For the cases $n_{1} \leq 10, n_{1}^{\prime}>10$ or $n_{1}>10, n_{1}^{\prime} \leq 10$, one just needs to combine the ideas in the above two situations together and use Theorem 6.2. Then we are done with $T_{1, m_{M, k_{0}}}^{E_{1}, E_{1}^{\prime}, 0}(f, g, h)$.

Now we turn to the study of the operators $T_{1, m_{M, k_{0}}}^{E_{2}, E_{2}^{\prime}, 0}$.

$$
\begin{aligned}
& \left\|T_{1, m_{M, k_{0}}}^{E_{2}, E_{2}^{\prime}, 0}(f, g, h)(x)\right\|_{r}^{r} \\
& =\| \sum_{\substack{I \subseteq\left(5 I^{0}\right)^{c} \\
I^{\prime} \subseteq\left(5 I^{\prime 0}\right)^{c}}} \sum_{\substack{J \in \mathcal{J} \\
\left|\omega_{J}^{3}\right| \leq\left|\omega_{I}^{2}\right|}} \frac{1}{|I|^{\frac{1}{2}}} \frac{1}{|J|^{\frac{1}{2}}} \frac{1}{\left|I^{\prime}\right|}\left\langle f, \phi_{I}^{1} \otimes \phi_{I^{\prime}}^{1}\right\rangle\left\langle g, \phi_{J}^{1} \otimes \phi_{I^{\prime}}^{2}\right\rangle \\
& \cdot\left\langle h, \phi_{J}^{2} \otimes \phi_{I^{\prime}}^{3}\right\rangle\left\langle\phi_{I}^{2}, \phi_{J}^{3}\right\rangle \phi_{I}^{3}\left(x_{1}\right) \phi_{I^{\prime}}^{4}\left(x_{2}\right) \varphi_{0}\left(x_{1}\right) \varphi_{0}^{\prime}\left(x_{2}\right) \|_{r}^{r} \\
& \lesssim \| \sum_{\substack{|n|,\left|n^{\prime}\right| \geq 5 \\
m \in \mathbb{Z}}} \sum_{\substack{i, j \geq 0 \\
i^{\prime} \geq 0}} \sum_{\substack{I \subseteq I_{n}, J \subseteq J_{m} \\
|I|=2^{-i},|J|=2^{-j}}} \sum_{\substack{I^{\prime} \subseteq I_{n^{\prime}} \\
\left|I^{\prime}\right|=2^{-i^{\prime}}}} \frac{1}{|I|^{\frac{1}{2}}} \frac{1}{|J|^{\frac{1}{2}}} \frac{1}{\left|I^{\prime}\right|}\left\langle f, \phi_{I}^{1} \otimes \phi_{I^{\prime}}^{1}\right\rangle\left\langle g, \phi_{J}^{1} \otimes \phi_{I^{\prime}}^{2}\right\rangle \\
& \cdot\left\langle h, \phi_{J}^{2} \otimes \phi_{I^{\prime}}^{3}\right\rangle\left\langle\phi_{I}^{2}, \phi_{J}^{3}\right\rangle \phi_{I}^{3}\left(x_{1}\right) \phi_{I^{\prime}}^{4}\left(x_{2}\right) \varphi_{0}\left(x_{1}\right) \varphi_{0}^{\prime}\left(x_{2}\right) \|_{r}^{r} \\
& \lesssim \sum_{\substack{|n|,\left|n^{\prime}\right| \geq 5 \\
m \in \mathbb{Z}}} \sum_{\substack{i, j \geq 0 \\
i^{\prime} \geq 0}} \sum_{\substack{I \subseteq I_{n}, J \subseteq J_{m} \\
|I|=2^{-i},|J|=2^{-j}}} \sum_{\substack{I^{\prime} \subseteq I_{n^{\prime}} \\
\left|I^{\prime}\right|=2^{-i^{\prime}}}}\left(\frac{1}{|I|}\left(1+\frac{\operatorname{dist}\left(I, I^{0}\right)}{|I|}\right)^{-M_{3}}\right. \\
& \cdot\left(1+\frac{\operatorname{dist}\left(I^{\prime}, I^{0}\right)}{\left|I^{\prime}\right|}\right)^{-M_{4}^{\prime}} \int_{\mathbb{R}}\left(1+\frac{\operatorname{dist}\left(x_{1}, I\right)}{|I|}\right)^{-M_{2}}\left(1+\frac{\operatorname{dist}\left(x_{1}, J\right)}{|J|}\right)^{-N_{3}} d x_{1} \\
& \left.\left\|f \chi_{I_{n}} \otimes \chi_{I_{n^{\prime}}}\right\|_{p_{1}}\left\|g \chi_{J_{m}} \otimes \chi_{I_{n^{\prime}}}\right\|_{p_{2}}\left\|h \chi_{J_{m}} \otimes \chi_{I_{n^{\prime}}}\right\|_{p_{3}}\right)^{r} \\
& \lesssim \sum_{\substack{|n|,\left|n^{\prime}\right| \geq 5 \\
m \in \mathbb{Z}}} \sum_{\substack{i, j \geq 0 \\
i^{\prime} \geq 0}} \sum_{\substack{I \subseteq I_{n}, J \subseteq J_{m} \\
|I|=2^{-i},|J|=2^{-j}}} \sum_{\substack{I^{\prime} \subseteq I_{n^{\prime}} \\
\left|I^{\prime}\right|=2^{-i^{\prime}}}}\left(2^{i}\left(1+2^{i}(|n|-2)\right)^{-M_{3}}\left(1+2^{i^{\prime}}\left(\left|n^{\prime}\right|-2\right)\right)^{-M_{4}^{\prime}}\right.
\end{aligned}
$$




$$
\begin{aligned}
& \left.\cdot(1+|n-m|)^{-N_{0}}\left\|f \chi_{I_{n}} \otimes \chi_{I_{n^{\prime}}}\right\|_{p_{1}}\left\|g \chi_{J_{m}} \otimes \chi_{I_{n^{\prime}}}\right\|_{p_{2}}\left\|h \chi_{J_{m}} \otimes \chi_{I_{n^{\prime}}}\right\|_{p_{3}}\right)^{r} \\
\lesssim & \sum_{\substack{|n|,\left|n^{\prime}\right| \geq 5 \\
m \in \mathbb{Z}}}\left((|n|-2)^{\frac{-M_{3}}{2}}\left(\left|n^{\prime}\right|-2\right)^{\frac{-M_{4}^{\prime}}{2}}(1+|n-m|)^{-N_{0}}\right. \\
& \left.\cdot\left\|f \chi_{I_{n}} \otimes \chi_{I_{n^{\prime}}}\right\|_{p_{1}}\left\|g \chi_{J_{m}} \otimes \chi_{I_{n^{\prime}}}\right\|_{p_{2}}\left\|h \chi_{J_{m}} \otimes \chi_{I_{n^{\prime}}}\right\|_{p_{3}}\right)^{r} \\
\lesssim & \left(\left\|f \tilde{\chi}_{R_{00}}\right\|_{p_{1}}\left\|g \tilde{\chi}_{R_{00}}\right\|_{p_{2}}\left\|h \tilde{\chi}_{R_{00}}\right\|_{p_{3}}\right)^{r} .
\end{aligned}
$$

For $T_{1, m_{M, k_{0}}}^{E_{1}, E_{2}^{\prime}, 0}(f, g, h)(x)$, we just need to combine the ideas for $T_{1, m_{M, k_{0}}}^{E_{1}, E_{1}^{\prime}, 0}(f, g, h)(x)$ and $T_{1, m_{M, k_{0}}}^{E_{2}, E_{2}^{\prime}, 0}(f, g, h)(x)$ together. More precisely, since we have $I \subseteq 5 I^{0}$ and $I^{\prime} \subseteq$ $\left(5 I^{0}\right)^{c}$, we can do the decomposition

$$
f(x)=\sum_{n_{1} \in \mathbb{Z}} f \cdot \chi_{I_{n_{1}}}\left(x_{1}\right), \quad g(x)=\sum_{n_{2} \in \mathbb{Z}} g \cdot \chi_{I_{n_{2}}}\left(x_{1}\right), \quad h(x)=\sum_{n_{3} \in \mathbb{Z}} h \cdot \chi_{I_{n_{3}}}\left(x_{1}\right) .
$$

As before first consider when $\left|n_{1}\right| \geq 10$.

$$
\begin{aligned}
& \left\|T_{1, m_{M, k_{0}}}^{E_{1}, E_{2}^{\prime}, 0}\left(f \chi_{I_{n_{1}}}, g \chi_{I_{n_{2}}}, h \chi_{I_{n_{3}}}\right)(x)\right\|_{r}^{r} \\
& \lesssim \sum_{\substack{\left|n^{\prime}\right| \geq 5 \\
m \in \mathbb{Z}}} \sum_{\substack{i, i^{\prime} \geq 0 \\
j \geq 0}} \sum_{\substack{I \subseteq 5 I^{0} \\
|I|=2^{-i}}} \sum_{\substack{I^{\prime} \subseteq I_{n^{\prime}} \\
\left|I^{\prime}\right|=2^{-i^{\prime}}}} \sum_{\substack{J \subseteq J_{m} \\
|J|=2^{-j}}}\left(\frac{1}{|I|}\left(1+\frac{\operatorname{dist}\left(I_{n_{1}}, I\right)}{|I|}\right)^{-M_{1}}\right. \\
& \left(1+\frac{\operatorname{dist}\left(I_{n_{2}}, J\right)}{|J|}\right)^{-N_{1}}\left(1+\frac{\operatorname{dist}\left(I_{n_{3}}, J\right)}{|J|}\right)^{-N_{2}}\left(1+\frac{\operatorname{dist}\left(I^{\prime}, I^{0}\right)}{\left|I^{\prime}\right|}\right)^{-M_{4}^{\prime}} \\
& \cdot \int_{\mathbb{R}}\left(1+\frac{\operatorname{dist}\left(x_{1}, I\right)}{|I|}\right)^{-M_{2}}\left(1+\frac{\operatorname{dist}\left(x_{1}, J\right)}{|J|}\right)^{-N_{3}} d x_{1} \\
& \left.\cdot\left\|f \chi_{I_{n_{1}}} \otimes \chi_{I_{n^{\prime}}}\right\|_{p_{1}}\left\|g \chi_{I_{n_{2}}} \otimes \chi_{I_{n^{\prime}}}\right\|_{p_{2}}\left\|h \chi_{I_{n_{3}}} \otimes \chi_{I_{n^{\prime}}}\right\|_{p_{3}}\right)^{r} \\
& \lesssim \sum_{\substack{\left|n^{\prime}\right| \geq 5 \\
m \in \mathbb{Z}}} \sum_{i, i^{\prime} \geq 0} \sum_{\substack{I \subseteq 5 I^{0} \\
j \geq 0}} \sum_{\substack{I^{\prime} \subseteq I_{n^{\prime}} \\
|I|=2^{-i}}} \sum_{\substack{J \subseteq J_{m} \\
\left|I^{\prime}\right|=2^{-i^{\prime}}|J|=2^{-j}}}\left(2^{i}\left(1+2^{i}\left(\left|n_{1}\right|-6\right)\right)^{-M_{1}}\left(1+2^{i^{\prime}}\left(\left|n^{\prime}\right|-2\right)\right)^{-M_{4}^{\prime}}\right. \\
& \cdot\left(1+2^{j}\left|m-n_{2}\right|\right)^{-N_{1}}\left(1+2^{j}\left|m-n_{3}\right|\right)^{-N_{2}}(1+|m|)^{-N_{0}} \\
& \left.\cdot\left\|f \chi_{I_{n_{1}}} \otimes \chi_{I_{n^{\prime}}}\right\|_{p_{1}}\left\|g \chi_{I_{n_{2}}} \otimes \chi_{I_{n^{\prime}}}\right\|_{p_{2}}\left\|h \chi_{I_{n_{3}}} \otimes \chi_{I_{n^{\prime}}}\right\|_{p_{3}}\right)^{r} \\
& \lesssim \sum_{\substack{\left|n^{\prime}\right| \geq 5 \\
m \in \mathbb{Z}}}\left(\left(\left|n_{1}\right|-6\right)^{-\frac{M_{1}}{2}}\left(\left|n^{\prime}\right|-2\right)^{-\frac{M_{1}^{\prime}}{2}}\left(1+\left|n_{2}\right|\right)^{-L}\left(1+\left|n_{3}\right|\right)^{-L}\right. \\
& \left.\cdot(1+|m|)^{-\frac{N_{0}}{2}}\left\|f \chi_{I_{n_{1}}} \otimes \chi_{I_{n^{\prime}}}\right\|_{p_{1}}\left\|g \chi_{I_{n_{2}}} \otimes \chi_{I_{n^{\prime}}}\right\|_{p_{2}}\left\|h \chi_{I_{n_{3}}} \otimes \chi_{I_{n^{\prime}}}\right\|_{p_{3}}\right)^{r} \\
& \lesssim \sum_{\left|n^{\prime}\right| \geq 5}\left(\left(\left|n_{1}\right|-6\right)^{-\frac{M_{1}}{4}}\left(\left|n^{\prime}\right|-2\right)^{-\frac{M_{1}^{\prime}}{6}}\left(1+\left|n_{2}\right|\right)^{-\frac{L}{2}}\left(1+\left|n_{3}\right|\right)^{-\frac{L}{2}}\right. \\
& \left.\cdot\left\|f \tilde{\chi}_{R_{00}}\right\|_{p_{1}}\left\|g \tilde{\chi}_{R_{00}}\right\|_{p_{2}}\left\|h \tilde{\chi}_{R_{00}}\right\|_{p_{3}}\right)^{r}
\end{aligned}
$$




$$
\lesssim\left(\left(\left|n_{1}\right|-6\right)^{-\frac{M_{1}}{4}}\left(1+\left|n_{2}\right|\right)^{-\frac{L}{2}}\left(1+\left|n_{3}\right|\right)^{-\frac{L}{2}}\left\|f \tilde{\chi}_{R_{00}}\right\|_{p_{1}}\left\|g \tilde{\chi}_{R_{00}}\right\|_{p_{2}}\left\|h \tilde{\chi}_{R_{00}}\right\|_{p_{3}}\right)^{r} .
$$

Thus,

$$
\begin{aligned}
& \left\|\sum_{\left|n_{1}\right|>10} \sum_{n_{2}, n_{3} \in \mathbb{Z}} T_{1, m_{M, k_{0}}}^{E_{1}, E_{2}^{\prime}, 0}\left(f \cdot \chi_{I_{n_{1}}}, g \cdot \chi_{I_{n_{2}}}, h \cdot \chi_{I_{n_{3}}}\right)(x)\right\|_{r}^{r} \\
\lesssim & \sum_{\left|n_{1}\right|>10} \sum_{n_{2}, n_{3} \in \mathbb{Z}}\left(\left(\left|n_{1}\right|-6\right)^{-\frac{M_{1}}{4}}\left(1+\left|n_{2}\right|\right)^{-\frac{L}{2}}\left(1+\left|n_{3}\right|\right)^{-\frac{L}{2}}\right. \\
& \left.\cdot\left\|f \tilde{\chi}_{R_{00}}\right\|_{p_{1}}\left\|g \tilde{\chi}_{R_{00}}\right\|_{p_{2}}\left\|h \tilde{\chi}_{R_{00}}\right\|_{p_{3}}\right)^{r} \\
\lesssim & \left(\left\|f \tilde{\chi}_{R_{00}}\right\|_{p_{1}}\left\|g \tilde{\chi}_{R_{00}}\right\|_{p_{2}}\left\|h \tilde{\chi}_{R_{00}}\right\|_{p_{3}}\right)^{r} .
\end{aligned}
$$

When $\left|n_{1}\right|<10$, as before we need Theorem 6.2 and some decay factors by (6.8), and the following holds

$$
\begin{aligned}
& \left\|\sum_{\substack{\left|n_{1}\right|<10 \\
n_{2}, n_{3} \in \mathbb{Z}}} T_{1, m_{M, k_{0}}}^{E_{1}, E_{2}^{\prime}, 0}\left(f \cdot \chi_{I_{n_{1}}}, g \cdot \chi_{I_{n_{2}}}, h \cdot \chi_{I_{n_{3}}}\right)(x)\right\|_{r}^{r} \\
\lesssim & \sum_{\substack {\left|n^{\prime}\right| \geq 5 \\
\begin{subarray}{c}{\left|n_{1}\right|<10 \\
n_{2}, n_{3} \in \mathbb{Z}{ | n ^ { \prime } | \geq 5 \\
\begin{subarray} { c } { | n _ { 1 } | < 1 0 \\
n _ { 2 } , n _ { 3 } \in \mathbb { Z } } }\end{subarray}}\left(1+\left|n_{2}\right|\right)^{-M^{\prime}}\left(1+\left|n_{3}\right|\right)^{-M^{\prime}}\left(\left|n^{\prime}\right|-2\right)^{-M^{\prime}} \\
\lesssim & \sum_{\left|n^{\prime}\right| \geq 5}^{E_{1}, E_{2}^{\prime}, 0}\left(f \cdot \chi_{n_{I_{1}} \mid<10}\left(1+\left|n_{2}\right|\right)^{-M^{\prime}} \cdot\left(1+\left|n_{3}\right|\right)^{-M^{\prime}}\left(\left|n^{\prime}\right|-2\right)^{-M^{\prime}}\right. \\
& \left.\| f \cdot \chi_{n_{n^{\prime}}}, g \cdot \chi_{I_{n_{2}}} \otimes \chi_{I_{n^{\prime}}}, h \cdot \chi_{I_{n_{3}}} \otimes \chi_{I_{n^{\prime}}}\right)(x)\left\|_{r}^{r}\right\| g \cdot \chi_{I_{n_{2}}} \otimes \chi_{I_{n^{\prime}}}\left\|_{p_{2}}\right\| h \cdot \chi_{I_{n_{3}}} \otimes \chi_{I_{n^{\prime}}} \|_{p_{3}} \\
\lesssim & \left\|f \tilde{\chi}_{R_{00}}\right\|_{p_{1}}\left\|g \tilde{\chi}_{R_{00}}\right\|_{p_{2}}\left\|h \tilde{\chi}_{R_{00}}\right\|_{p_{3}} .
\end{aligned}
$$

where $M^{\prime}$ is sufficiently large. Then we are done with $T_{1, m_{M, k_{0}}}^{E_{1}, E_{1}^{\prime}, 0}$.

Moreover, it is obvious that $T_{1, m_{M, k_{0}}}^{E_{2} E_{1}^{\prime}, 0}$ can be treated similarly. We omit the details here. Now we are done with $T_{1, m_{M, k_{0}}}^{E, E^{\prime}, 0}(f, g, h)(x)$. Now we have proved the desired estimate for the operators $T_{a b}^{E, E^{\prime}, 0}(f, g, h)(x)$.

6.3. $T_{a b}^{E, G^{\prime}, 0}, T_{a b}^{G, E^{\prime}, 0}$.

Consider

$$
\begin{gathered}
T_{a b}^{E, G^{\prime}, 0}(f, g, h)(x) \\
\approx \sum_{I \in \mathcal{I}} \sum_{I^{\prime} \in \mathcal{I}^{\prime}} \sum_{\substack{J \in \mathcal{J} \\
\left|\omega_{J}^{3}\right| \leq\left|\omega_{I}^{2}\right| \\
\\
\cdot\left\langle\phi_{J}^{3}, \phi_{I}^{2}\right\rangle \phi_{I}^{3}\left(x_{1}\right) \phi_{I^{\prime}}^{4}\left(x_{2}\right) \varphi_{0}\left(x_{1}\right) \varphi_{0}^{\prime}\left(x_{2}\right)}} \frac{1}{|I|^{\frac{1}{2}}} \frac{1}{\left|I^{\prime}\right|}\left\langle f, \phi_{I}^{1} \otimes \phi_{I^{\prime}}^{1}\right\rangle\left\langle g, \phi_{J}^{1} \otimes \phi_{I^{\prime}}^{2}\right\rangle\left\langle h, \phi_{I}^{2} \otimes \phi_{I^{\prime}}^{3}\right\rangle
\end{gathered}
$$




$$
\begin{gathered}
+\left(\int_{\mathbb{R}^{6}} m_{M, k_{0}}^{1}\left(\xi_{1}, \eta_{1}, \zeta_{1}\right)\left(\sum_{k \geq 0} \phi_{k}^{1}\left(\xi_{2}\right) \phi_{k}^{2}\left(\eta_{2}\right) \phi_{k}^{3}\left(\zeta_{2}\right)\right)\right. \\
\cdot \hat{f}(\xi) \hat{g}(\eta) \hat{h}(\zeta) d \xi d \eta d \zeta) \varphi_{0}\left(x_{1}\right) \varphi_{0}^{\prime}\left(x_{2}\right) \\
:=T_{a b}^{E, G^{\prime}, 0,1}+T_{a b}^{E, G^{\prime}, 0,2},
\end{gathered}
$$

where $m_{M, k_{0}}^{1}$ is as described in Lemma 6.1. Also note

$$
\left|\partial_{\xi_{2}}^{\alpha} \partial_{\eta_{2}}^{\beta} \partial_{\zeta_{2}}^{\gamma}\left(\sum_{k \geq 0} \phi_{k}^{1}\left(\xi_{2}\right) \phi_{k}^{2}\left(\eta_{2}\right) \phi_{k}^{3}\left(\zeta_{2}\right)\right)\right| \lesssim \frac{1}{\left(1+\left|\xi_{2}\right|+\left|\eta_{2}\right|+\left|\zeta_{2}\right|\right)^{\alpha+\beta+\gamma}}
$$

for sufficiently many indices. Then the desired estimate for $T_{a b}^{E, G^{\prime}, 0,1}$ follows from the same argument as for $T_{1, m_{M, k_{0}}^{E}, E^{\prime}, 0}$, and the estimate for $T_{a b}^{E, G^{\prime}, 0,2}$ follows from Theorem 1.5 .

Having treated all the cases in Theorem 5.1, the proof of Theorem 1.10 is concluded.

\section{Appendix A.}

Here we give some details about the reductions used in the Leibniz rule. Here we will still use the notations introduced in Section 3. We start from the reduction of $D_{1}^{\beta_{1}} D_{2}^{\beta_{2}}(g h)$, and this part has appeared in [20 22]. We recall some arguments here. Let

$$
\begin{aligned}
& 1\left(\eta_{1}, \eta_{2}, \zeta_{1}, \zeta_{2}\right) \\
= & \left(\sum_{k_{1}} \widehat{\psi}_{k_{1}}\left(\eta_{1}\right) \sum_{l_{1}} \widehat{\psi}_{l_{1}}\left(\zeta_{1}\right)\right)\left(\sum_{k_{2}} \widehat{\psi}_{k_{2}}\left(\eta_{2}\right) \sum_{l_{2}} \widehat{\psi}_{l_{2}}\left(\zeta_{2}\right)\right) \\
= & \left(\sum_{k_{1}} \widehat{\varphi}_{k_{1}}\left(\eta_{1}\right) \widehat{\psi}_{k_{1}}\left(\zeta_{1}\right)+\sum_{k_{1}} \widehat{\psi}_{k_{1}}\left(\eta_{1}\right) \widehat{\psi}_{k_{1}}\left(\zeta_{1}\right)+\sum_{k_{1}} \widehat{\psi}_{k_{1}}\left(\eta_{1}\right) \widehat{\varphi}_{k_{1}}\left(\zeta_{1}\right)\right), \\
& \cdot\left(\sum_{k_{2}} \widehat{\varphi}_{k_{2}}\left(\eta_{2}\right) \widehat{\psi}_{k_{2}}\left(\zeta_{2}\right)+\sum_{k_{2}} \widehat{\psi}_{k_{2}}\left(\eta_{2}\right) \widehat{\psi}_{k_{2}}\left(\zeta_{2}\right)+\sum_{k_{2}} \widehat{\psi}_{k_{2}}\left(\eta_{2}\right) \widehat{\varphi}_{k_{2}}\left(\zeta_{2}\right)\right)
\end{aligned}
$$

where $\widehat{\varphi}_{k_{i}}=\sum_{l_{i} \leq k_{i}-100} \widehat{\psi}_{l_{i}}$, and $\widehat{\psi}_{k_{i}}$ may actually represent a function $\sum_{k_{i}-100 \leq l_{i} \leq k_{i}+100} \widehat{\psi}_{l_{i}}$ (we don't distinguish them since their supports are both away from 0 ) for $i=1,2$. Then $g \cdot h$ can be written as a summation of the terms like, for example,

$$
g \cdot h=\sum_{k_{1}, k_{2}}\left(g *\left(\psi_{k_{1}} \otimes \psi_{k_{2}}\right)\right) \cdot\left(h *\left(\varphi_{k_{1}} \otimes \varphi_{k_{2}}\right)\right) .
$$

Moreover, it can actually be rewritten as the following bi-parameter paraproduct

$$
\Pi(g, h)=\sum_{k_{1}, k_{2}}\left(\left(\left(g *\left(\psi_{k_{1}} \otimes \psi_{k_{2}}\right)\right) \cdot\left(h *\left(\varphi_{k_{1}} \otimes \varphi_{k_{2}}\right)\right)\right) * \widetilde{\psi}_{k_{1}} \otimes \tilde{\psi}_{k_{2}}\right),
$$


where $\psi_{k_{1}} \otimes \psi_{k_{2}}=\psi_{k_{1}}\left(x_{1}\right) \psi_{k_{2}}\left(x_{2}\right)$, and $\widehat{\widetilde{\psi}}_{k_{i}}$ is an inserted Schwartz function whose support is away from 0 and satisfying

$$
\widehat{\tilde{\psi}}_{k_{i}}=1 \quad \text { on } \quad \operatorname{supp} \widehat{\psi}_{k_{i}}+\operatorname{supp} \widehat{\varphi}_{k_{i}}, \quad i=1,2 .
$$

Note that in section 3 , we simply use $\psi_{k}$ instead of $\widetilde{\psi}_{k}$, since they are of the same type, i.e, they are supported on $\left\{u: c 2^{k-1} \leq|u| \leq c 2^{k+1}\right\}$ for appropriate constants $c$, which are away from 0 .

Now the differentiation $D_{1}^{\beta_{1}} D_{2}^{\beta_{2}}(g, h)$ can be written as a summation of the terms like $D_{1}^{\beta_{1}} D_{2}^{\beta_{2}} \Pi(g, h)$, which can be written as

$$
\begin{aligned}
& D_{1}^{\beta_{1}} D_{2}^{\beta_{2}} \Pi(g, h) \\
&= \sum_{k_{1}, k_{2}}\left(\left(\left(g *\left(\psi_{k_{1}} \otimes \psi_{k_{2}}\right)\right) \cdot\left(h *\left(\varphi_{k_{1}} \otimes \varphi_{k_{2}}\right)\right)\right) * D_{1}^{\beta_{1}} D_{2}^{\beta_{2}}\left(\widetilde{\psi}_{k_{1}} \otimes \widetilde{\psi}_{k_{2}}\right)\right) \\
&=\left.\sum_{k_{1}, k_{2}}\left(\left(g *\left(\psi_{k_{1}} \otimes \psi_{k_{2}}\right)\right) \cdot\left(h *\left(\varphi_{k_{1}} \otimes \varphi_{k_{2}}\right)\right)\right) * 2^{k_{1} \beta_{1}} 2^{k_{2} \beta_{2}}\left({\widetilde{\psi^{\prime}}}_{k_{1}} \otimes{\widetilde{\psi^{\prime}}}_{k_{2}}\right)\right) \\
&= \sum_{k_{1}, k_{2}}\left(\left(\left(g *\left(2^{k_{1} \beta_{1}} \psi_{k_{1}} \otimes 2^{k_{2} \beta_{2}} \psi_{k_{2}}\right)\right) \cdot\left(h *\left(\varphi_{k_{1}} \otimes \varphi_{k_{2}}\right)\right)\right) *\left({\widetilde{\psi^{\prime}}}_{k_{1}} \otimes{\widetilde{\psi^{\prime}}}_{k_{2}}\right)\right) \\
&= \sum_{k_{1}, k_{2}}\left(\left(\left(g *\left(D_{1}^{\beta_{1}} \psi_{k_{1}}^{\prime \prime} \otimes D_{2}^{\beta_{2}} \psi_{k_{2}}^{\prime \prime}\right)\right) \cdot\left(h *\left(\varphi_{k_{1}} \otimes \varphi_{k_{2}}\right)\right)\right) *\left({\widetilde{\psi^{\prime}}}_{k_{1}} \otimes{\widetilde{\psi^{\prime}}}_{k_{2}}\right)\right) \\
&= \sum_{k_{1}, k_{2}}\left(\left(\left(D_{1}^{\beta_{1}} D_{2}^{\beta_{2}} g *\left(\psi_{k_{1}}^{\prime \prime} \otimes \psi_{k_{2}}^{\prime \prime}\right)\right) \cdot\left(h *\left(\varphi_{k_{1}} \otimes \varphi_{k_{2}}\right)\right)\right) *\left({\widetilde{\psi^{\prime}}}_{k_{1}} \otimes{\widetilde{\psi^{\prime}}}_{k_{2}}\right)\right) \\
&:= \Pi\left(D_{1}^{\beta_{1}} D_{2}^{\beta_{2}} g, h\right), \\
& \text { where } \widehat{\widetilde{\psi}}_{k_{i}}(u):=\widehat{\widetilde{\psi}}_{k_{i}}(u)\left|\frac{u}{2^{k_{i}}}\right|^{\beta_{i}},{\widehat{\psi^{\prime \prime}}}_{k_{i}}(u):=\widehat{\psi}_{k_{i}}(u)\left(\frac{2^{k_{i}}}{|u|}\right)^{\beta_{i}} \text { for } i=1,2 .
\end{aligned}
$$

Note that the idea is to "move" the differential operator to appropriate functions. In the above expressions we finally apply the differential operators to $g$ because the associated convolution has both $\psi$ type functions involved. That allows us to multiply or divide them by functions $|u|^{\beta_{i}}$ as we need, i.e, we can always make $\psi^{\prime \prime}$ smooth.

Using a similar idea, we decompose $f$ as

$$
f=\sum_{j_{1}} \sum_{j_{2}}\left(f *\left(\psi_{j_{1}} \otimes \psi_{j_{2}}\right)\right)
$$

then $f \cdot g \cdot h$ can be written as a summation of the terms like, for example,

$$
\sum_{j_{1}} \sum_{j_{2}}\left(f *\left(\psi_{j_{1}} \otimes \psi_{j_{2}}\right)\right) \sum_{l_{1} \ll k_{1}} \sum_{l_{2} \ll k_{2}}\left(g *\left(\psi_{k_{1}} \otimes \psi_{k_{2}}\right)\right) \cdot\left(h *\left(\psi_{l_{1}} \otimes \psi_{\ell_{2}}\right)\right)
$$




$$
\begin{gathered}
=\left(\sum_{j_{1} \ll k_{1}}+\sum_{j_{1} \simeq k_{1}}+\sum_{j_{1} \gg k_{1}}\right)\left(\sum_{j_{2} \ll k_{2}}+\sum_{j_{2} \simeq k_{2}}+\sum_{j_{2} \gg k_{2}}\right) \sum_{l_{1} \ll k_{1}} \sum_{l_{2} \ll k_{2}} \\
\cdot\left(f *\left(\psi_{j_{1}} \otimes \psi_{j_{2}}\right)\right)\left(g *\left(\psi_{k_{1}} \otimes \psi_{k_{2}}\right)\right) \cdot\left(h *\left(\psi_{l_{1}} \otimes \psi_{\ell_{2}}\right)\right) .
\end{gathered}
$$

In the above summation, let's take a look at the following part with $\sum_{k_{1} \ll j_{1}} \sum_{k_{2} \ll j_{2}}$,

$$
\begin{aligned}
& \int_{\mathbb{R}^{6}} \sum_{l_{1} \ll k_{1} \ll j_{1}} \sum_{l_{2} \ll k_{2} \ll j_{2}}\left(\widehat{\psi}_{j_{1}}\left(\xi_{1}\right) \widehat{\psi}_{j_{2}}\left(\xi_{2}\right) \widehat{\psi}_{k_{1}}\left(\eta_{1}\right) \widehat{\psi}_{k_{2}}\left(\eta_{2}\right) \widehat{\psi}_{l_{1}}\left(\zeta_{1}\right) \widehat{\psi}_{l_{2}}\left(\zeta_{2}\right)\right) \\
& \cdot \hat{f}\left(\xi_{1}, \xi_{2}\right) \hat{g}\left(\eta_{1}, \eta_{2}\right) \hat{h}\left(\zeta_{1}, \zeta_{2}\right) e^{2 \pi i(\xi+\eta+\zeta) x} d \xi d \eta d \zeta \\
&:=\int_{\mathbb{R}^{6}} m(\xi, \eta, \zeta) \hat{f}\left(\xi_{1}, \xi_{2}\right) \hat{g}\left(\eta_{1}, \eta_{2}\right) \hat{h}\left(\zeta_{1}, \zeta_{2}\right) e^{2 \pi i(\xi+\eta+\zeta) x} d \xi d \eta d \zeta
\end{aligned}
$$

where the symbol can be rewritten as

$$
\begin{aligned}
& m(\xi, \eta, \zeta) \\
= & \left(\sum_{j_{1}} \sum_{j_{2}} \widehat{\psi}_{j_{1}}\left(\xi_{1}\right) \widehat{\psi}_{j_{2}}\left(\xi_{2}\right) \widehat{\varphi}_{j_{1}}\left(\eta_{1}+\zeta_{1}\right) \widehat{\varphi}_{j_{2}}\left(\eta_{2}+\zeta_{2}\right) \widehat{\psi}_{j_{1}}\left(\xi_{1}+\eta_{1}+\zeta_{1}\right) \widehat{\psi}_{j_{2}}\left(\xi_{2}+\eta_{2}+\zeta_{2}\right)\right) \\
& \cdot\left(\sum_{k_{1} \ll j_{1}} \sum_{k_{2} \ll j_{2}} \widehat{\psi}_{k_{1}}\left(\eta_{1}\right) \widehat{\psi}_{k_{2}}\left(\eta_{2}\right) \widehat{\varphi}_{k_{1}}\left(\zeta_{1}\right) \widehat{\varphi}_{k_{2}}\left(\zeta_{2}\right) \widehat{\psi}_{k_{1}}\left(\eta_{1}+\zeta_{1}\right) \widehat{\psi}_{k_{2}}\left(\eta_{2}+\zeta_{2}\right)\right),
\end{aligned}
$$

where some appropriate $\varphi$ type functions and $\psi$ type functions are inserted as before, based on the supports of the functions. With the above, (A.5) becomes

$$
\sum_{j_{1}, j_{2}}\left(\left(f *\left(\psi_{j_{1}} \otimes \psi_{j_{2}}\right)\right)\left(\Pi_{1}(g, h) *\left(\varphi_{j_{1}} \otimes \varphi_{j_{2}}\right)\right)\right) *\left(\psi_{j_{1}} \otimes \psi_{j_{2}}\right),
$$

where

$$
\Pi_{1}(g, h)=\sum_{k_{1} \ll j_{1}} \sum_{k_{2} \ll j_{2}}\left(\left(g *\left(\psi_{k_{1}} \otimes \psi_{k_{2}}\right)\right)\left(h *\left(\varphi_{k_{1}} \otimes \varphi_{k_{2}}\right)\right)\right) *\left(\psi_{k_{1}} \otimes \psi_{k_{2}}\right) .
$$

Recall by (A.3),$D_{1}^{\beta_{1}} D_{2}^{\beta_{2}} \Pi_{1}(g, h)$ can be written as a summation of the terms like, for example,

$$
\sum_{k_{1} \ll j_{1}} \sum_{k_{2} \ll j_{2}}\left(\left(D_{1}^{\beta_{1}} D_{2}^{\beta_{2}} g *\left(\psi_{k_{1}}^{\prime \prime} \otimes \psi_{k_{2}}^{\prime \prime}\right)\right) \cdot\left(h *\left(\varphi_{k_{1}} \otimes \varphi_{k_{2}}\right)\right)\right) *\left(\psi_{k_{1}}^{\prime} \otimes \psi_{k_{2}}^{\prime}\right)
$$

Then when we apply the differential operator $D_{1}^{\alpha_{1}} D_{2}^{\alpha_{2}}$ to $f \cdot D_{1}^{\beta_{1}} D_{2}^{\beta_{2}} \Pi_{1}(g, h)$,

$$
\begin{aligned}
& D_{1}^{\alpha_{1}} D_{2}^{\alpha_{2}}\left(\sum_{j_{1}, j_{2}}\left(\left(f *\left(\psi_{j_{1}} \otimes \psi_{j_{2}}\right)\right)\left(D_{1}^{\beta_{1}} D_{2}^{\beta_{2}} \Pi_{1}(g, h) *\left(\varphi_{j_{1}} \otimes \varphi_{j_{2}}\right)\right)\right) *\left(\psi_{j_{1}} \otimes \psi_{j_{2}}\right)\right) \\
= & \sum_{j_{1}, j_{2}}\left(\left(f *\left(\psi_{j_{1}} \otimes \psi_{j_{2}}\right)\right)\left(D_{1}^{\beta_{1}} D_{2}^{\beta_{2}} \Pi_{1}(g, h) *\left(\varphi_{j_{1}} \otimes \varphi_{j_{2}}\right)\right)\right) * D_{1}^{\alpha_{1}} D_{2}^{\alpha_{2}}\left(\psi_{j_{1}} \otimes \psi_{j_{2}}\right)
\end{aligned}
$$




$$
\begin{aligned}
& =\sum_{j_{1}, j_{2}}\left(\left(f *\left(\psi_{j_{1}} \otimes \psi_{j_{2}}\right)\right)\left(D_{1}^{\beta_{1}} D_{2}^{\beta_{2}} \Pi_{1}(g, h) *\left(\varphi_{j_{1}} \otimes \varphi_{j_{2}}\right)\right)\right) * 2^{j_{1} \alpha_{1}} 2^{j_{2} \alpha_{2}}\left(\psi_{j_{1}}^{\prime} \otimes \psi_{j_{2}}^{\prime}\right) \\
& =\sum_{j_{1}, j_{2}}\left(\left(f *\left(2^{j_{1} \alpha_{1}} \psi_{j_{1}} \otimes 2^{j_{2} \alpha_{2}} \psi_{j_{2}}\right)\right)\left(D_{1}^{\beta_{1}} D_{2}^{\beta_{2}} \Pi_{1}(g, h) *\left(\varphi_{j_{1}} \otimes \varphi_{j_{2}}\right)\right)\right) *\left(\psi_{j_{1}}^{\prime} \otimes \psi_{j_{2}}^{\prime}\right) \\
& =\sum_{j_{1}, j_{2}}\left(\left(f *\left(D_{1}^{\alpha_{1}} \psi_{j_{1}}^{\prime \prime} \otimes D_{2}^{\alpha_{2}} \psi_{j_{2}}^{\prime \prime}\right)\right)\left(D_{1}^{\beta_{1}} D_{2}^{\beta_{2}} \Pi_{1}(g, h) *\left(\varphi_{j_{1}} \otimes \varphi_{j_{2}}\right)\right)\right) *\left(\psi_{j_{1}}^{\prime} \otimes \psi_{j_{2}}^{\prime}\right) \\
& =\sum_{j_{1}, j_{2}}\left(\left(D_{1}^{\alpha_{1}} D_{2}^{\alpha_{2}} f *\left(\psi_{j_{1}}^{\prime \prime} \otimes \psi_{j_{2}}^{\prime \prime}\right)\right)\left(D_{1}^{\beta_{1}} D_{2}^{\beta_{2}} \Pi_{1}(g, h) *\left(\varphi_{j_{1}} \otimes \varphi_{j_{2}}\right)\right)\right) *\left(\psi_{j_{1}}^{\prime} \otimes \psi_{j_{2}}^{\prime}\right) \\
& :=\sum_{j_{1}, j_{2}}\left(\left(D_{1}^{\alpha_{1}} D_{2}^{\alpha_{2}} f *\left(\psi_{j_{1}}^{\prime \prime} \otimes \psi_{j_{2}}^{\prime \prime}\right)\right)\left(D_{1}^{\beta_{1}} D_{2}^{\beta_{2}} \Pi_{1}(g, h) *\left(\varphi_{j_{1}} \otimes \varphi_{j_{2}}\right)\right)\right) *\left(\psi_{j_{1}}^{\prime} \otimes \psi_{j_{2}}^{\prime}\right) .
\end{aligned}
$$

Based on the above form, and by removing some inserted and reinserting appropriate functions as before, we can write the associated symbol as

$$
\begin{aligned}
& \left(\sum_{j_{1}} \sum_{j_{2}} \widehat{\psi}_{j_{1}}^{\prime \prime}\left(\xi_{1}\right) \widehat{\psi}_{j_{2}}^{\prime \prime}\left(\xi_{2}\right) \widehat{\psi}_{j_{1}}^{\prime}\left(\xi_{1}+\eta_{1}+\zeta_{1}\right) \widehat{\psi}_{j_{2}}^{\prime}\left(\xi_{2}+\eta_{2}+\zeta_{2}\right)\right) \\
& \cdot\left(\sum_{k_{1} \ll j_{1}} \sum_{k_{2} \ll j_{2}} \widehat{\psi}_{k_{1}}^{\prime \prime}\left(\eta_{1}\right) \widehat{\psi}_{k_{2}}^{\prime \prime}\left(\eta_{2}\right) \widehat{\varphi}_{k_{1}}\left(\zeta_{1}\right) \widehat{\varphi}_{k_{2}}\left(\zeta_{2}\right) \widehat{\psi}_{k_{1}}^{\prime}\left(\eta_{1}+\zeta_{1}\right) \widehat{\psi}_{k_{2}}^{\prime}\left(\eta_{2}+\zeta_{2}\right)\right) \\
= & \left(\sum_{j_{1}} \sum_{j_{2}} \widehat{\psi}_{j_{1}}^{\prime \prime}\left(\xi_{1}\right) \widehat{\psi}_{j_{2}}^{\prime \prime}\left(\xi_{2}\right) \widehat{\varphi}_{j_{1}}\left(\eta_{1}\right) \widehat{\varphi}_{j_{2}}\left(\eta_{2}\right) \widehat{\psi}_{j_{1}}^{\prime}\left(\xi_{1}+\eta_{1}+\zeta_{1}\right) \widehat{\psi}_{j_{2}}^{\prime}\left(\xi_{2}+\eta_{2}+\zeta_{2}\right)\right) \\
= & \cdot\left(\sum_{k_{1} \ll j_{1}} \sum_{k_{2} \ll j_{2}} \widehat{\psi}_{k_{1}}^{\prime \prime}\left(\eta_{1}\right) \widehat{\psi}_{k_{2}}^{\prime \prime}\left(\eta_{2}\right) \widehat{\varphi}_{k_{1}}\left(\zeta_{1}\right) \widehat{\psi}_{k_{2}}\left(\zeta_{2}\right)\right) \\
& \cdot\left(\sum_{k_{1}} \sum_{k_{2}} \widehat{\psi}_{k_{1}}\left(\xi_{1}\right) \widehat{\psi}_{j_{2}}^{\prime \prime}\left(\xi_{2}\right) \widehat{\varphi}_{j_{1}}\left(\eta_{1}\right) \widehat{\psi}_{j_{2}}\left(\eta_{2}\right) \widehat{\psi}_{j_{1}}^{\prime \prime}\left(\eta_{2}\right) \widehat{\varphi}_{k_{1}}\left(\zeta_{1}\right) \widehat{\varphi}_{k_{2}}\left(\zeta_{2}\right)\right) \\
= & m_{1}(\xi, \eta, \zeta) \eta_{2}(\eta, \zeta),
\end{aligned}
$$

where in the last estimate we are able to ignore the restriction $k_{i} \ll j_{i}$ when taking the summation, because $\varphi_{j_{i}}$ is a properly chosen function such that $\widehat{\varphi}_{j_{i}}\left(\eta_{i}\right) \widehat{\psi}_{k_{i}}^{\prime \prime}\left(\eta_{i}\right)=0$ if the restriction is not satisfied $(i=1,2)$.

Note that $m_{1}$ and $m_{2}$ belong to the classical symbols $\mathcal{B M}\left(\mathbb{R}^{6}\right)$ and $\mathcal{B M}\left(\mathbb{R}^{4}\right)$ respectively. Then the bound

$$
\left\|D_{1}^{\alpha_{1}} D_{2}^{\alpha_{2}} f\right\|_{L^{p_{1}}} \cdot\left\|D_{1}^{\beta_{1}} D_{2}^{\beta_{2}} g\right\|_{L^{q_{1}}} \cdot\|h\|_{L^{s_{1}}}
$$


follows from the boundedness in Theorem 1.7. In fact, the other terms in the Leibniz estimate (3.3) can be obtained in the similar way. Taking the decomposition $g \cdot h$ for example, recall that the above argument is based on one of the terms in the decomposition of $g \cdot h$, i.e. (A.2). However, the are actually 9 terms in the decomposition, as indicated by (A.1). The similar thing happens after $f$ is introduced. In short, among the rest of these terms, parts of them are covered by Theorem 1.7. The other parts can take different forms, based on the "positions" of the $\psi$ functions $\varphi$ functions, and the $L^{r}$ estimate of them gives the other 15 pieces in (3.3). An example has been given earlier.

However, the other part of those remaining terms cannot be treated as the operator $T_{m_{1}, m_{2}}$. As we mentioned in Section 3 , these terms actually correspond to the multiplier $T_{m_{3}, m_{4}}$. More precisely, if we check following term in the decomposition of $f \cdot g \cdot h$,

$$
\sum_{l_{1} \ll k_{1} \ll j_{1}} \sum_{j_{2} \ll k_{2} \ll l_{2}}\left(f *\left(\psi_{j_{1}} \otimes \psi_{j_{2}}\right)\right)\left(g *\left(\psi_{k_{1}} \otimes \psi_{k_{2}}\right)\right)\left(h *\left(\psi_{l_{1}} \otimes \psi_{\ell_{2}}\right)\right),
$$

we can see it is actually a Fourier multiplier:

$$
\begin{aligned}
& \int_{\mathbb{R}^{6}} \sum_{l_{1} \ll k_{1} \ll j_{1}} \sum_{l_{2} \gg k_{2} \gg j_{2}}\left(\widehat{\psi}_{j_{1}}\left(\xi_{1}\right) \widehat{\psi}_{j_{2}}\left(\xi_{2}\right) \widehat{\psi}_{k_{1}}\left(\eta_{1}\right) \widehat{\psi}_{k_{2}}\left(\eta_{2}\right) \widehat{\psi}_{l_{1}}\left(\zeta_{1}\right) \widehat{\psi}_{l_{2}}\left(\zeta_{2}\right)\right) \\
& \cdot \hat{f}\left(\xi_{1}, \xi_{2}\right) \hat{g}\left(\eta_{1}, \eta_{2}\right) \hat{h}\left(\zeta_{1}, \zeta_{2}\right) e^{2 \pi i(\xi+\eta+\zeta) x} d \xi d \eta d \zeta \\
&:=\int_{\mathbb{R}^{6}} m^{\prime}(\xi, \eta, \zeta) \hat{f}\left(\xi_{1}, \xi_{2}\right) \hat{g}\left(\eta_{1}, \eta_{2}\right) \hat{h}\left(\zeta_{1}, \zeta_{2}\right) e^{2 \pi i(\xi+\eta+\zeta) x} d \xi d \eta d \zeta
\end{aligned}
$$

Using the trick of inserting terms as before, we can write

$$
\begin{aligned}
& m^{\prime}(\xi, \eta, \zeta) \\
= & \left(\sum_{j_{1}} \sum_{k_{2}} \widehat{\psi}_{j_{1}}\left(\xi_{1}\right) \widehat{\varphi}_{k_{2}}\left(\xi_{2}\right) \widehat{\varphi}_{j_{1}}\left(\eta_{1}+\zeta_{1}\right) \widehat{\psi}_{k_{2}}\left(\eta_{2}\right) \widehat{\psi}_{j_{1}}\left(\xi_{1}+\eta_{1}+\zeta_{1}\right) \widehat{\psi}_{k_{2}}\left(\xi_{2}+\eta_{2}\right)\right) \\
& \cdot\left(\sum_{k_{1} \ll j_{1}} \sum_{l_{2} \gg k_{2}} \widehat{\psi}_{k_{1}}\left(\eta_{1}\right) \widehat{\varphi}_{l_{2}}\left(\xi_{2}+\eta_{2}\right) \widehat{\varphi}_{k_{1}}\left(\zeta_{1}\right) \widehat{\psi}_{l_{2}}\left(\zeta_{2}\right) \widehat{\psi}_{k_{1}}\left(\eta_{1}+\zeta_{1}\right) \widehat{\psi}_{l_{2}}\left(\xi_{2}+\eta_{2}+\zeta_{2}\right)\right) \\
= & \left(\sum_{j_{1}} \sum_{l_{2}} \widehat{\psi}_{j_{1}}\left(\xi_{1}\right) \widehat{\varphi}_{j_{1}}\left(\eta_{1}+\zeta_{1}\right) \widehat{\psi}_{j_{1}}\left(\xi_{1}+\eta_{1}+\zeta_{1}\right) \widehat{\varphi}_{l_{2}}\left(\xi_{2}+\eta_{2}\right) \widehat{\psi}_{l_{2}}\left(\zeta_{2}\right) \widehat{\psi}_{l_{2}}\left(\xi_{2}+\eta_{2}+\zeta_{2}\right)\right) \\
& \cdot\left(\sum_{k_{1} \ll j_{1}} \sum_{k_{2} \ll l_{2}} \widehat{\psi}_{k_{1}}\left(\eta_{1}\right) \widehat{\varphi}_{k_{1}}\left(\zeta_{1}\right) \widehat{\psi}_{k_{1}}\left(\eta_{1}+\zeta_{1}\right) \widehat{\varphi}_{k_{2}}\left(\xi_{2}\right) \widehat{\psi}_{k_{2}}\left(\eta_{2}\right) \widehat{\psi}_{k_{2}}\left(\xi_{2}+\eta_{2}\right)\right) .
\end{aligned}
$$


Note that in this case $m^{\prime}$ is essentially a symbol

$$
m^{\prime}(\xi, \eta, \zeta)=\left(m^{\prime, 1}\left(\xi_{1}, \eta_{1}, \zeta_{1}\right) m^{\prime, 2}\left(\eta_{1}, \zeta_{1}\right)\right)\left(m^{\prime, 3}\left(\xi_{2}, \eta_{2}, \zeta_{2}\right) m^{\prime, 4}\left(\xi_{2}, \eta_{2}\right)\right),
$$

with $m^{\prime, 1}, m^{\prime, 3} \in \mathcal{M}\left(\mathbb{R}^{3}\right)$ and $m^{\prime, 2}, m^{\prime, 4} \in \mathcal{M}\left(\mathbb{R}^{2}\right)$. Strictly speaking, $m^{\prime}$ is not exactly a symbol having the form $m_{4}(\eta, \zeta) m_{3}(\xi, \eta)$, with $m_{3}, m_{4}$ in $\mathcal{B} \mathcal{M}\left(\mathbb{R}^{4}\right)$. However, it is a fact that these two symbols share the same difficulty in obtaining their Hölder-type estimates. Thus, without loss of generality, we treat them as if they were the same. As before, after we apply those differential operators, since the "types" of those $\psi$ and $\varphi$ functions do not change, the form of the associated symbol won't change, either. This means that the Leibniz rule for these terms will follow from the Hölder type $L^{r}$ estimate of $T_{m_{3}, m_{4}}$. Another difficulty in this case is that an analogue of (A.7) will have a more complicated form, another challenge in obtaining the desired estimate.

\section{Appendix B.}

In this section, we obtain the mixed norm estimates when $1<p, p_{2}, p_{3}, q_{2}, q_{3}<\infty$ in [26] via looking at the bi-parameter multilinear operators (1.5) and the reduced operator (1.8). We consider (1.8) under the tensor product setting first.

Proposition B.1. Let $g(x)=g_{1}\left(x_{1}\right) \otimes g_{2}\left(x_{2}\right), h(x)=h_{1}\left(x_{1}\right) \otimes h_{2}\left(x_{2}\right)$, and $\frac{1}{p}+$ $\frac{1}{p_{2}}+\frac{1}{p_{3}}=\frac{1}{p}+\frac{1}{q_{2}}+\frac{1}{q_{3}}=\frac{1}{r}$. Then (1.8) maps $L^{p} \times L_{x_{1}}^{p_{2}}\left(L_{x_{2}}^{q_{2}}\right) \times L_{x_{1}}^{p_{3}}\left(L_{x_{2}}^{q_{3}}\right) \rightarrow L^{r}$, with $0<r<\infty$ and $1<p, p_{2}, p_{3}, q_{2}, q_{3}<\infty$.

Proof. Using the tensor products, (1.8) becomes

$$
\begin{aligned}
& \int_{\mathbb{R}^{6}} m^{\prime}\left(\xi_{1}, \eta_{1}, \zeta_{1}\right) m^{\prime \prime}\left(\eta_{2}, \zeta_{2}\right) \hat{f}(\xi) \hat{g}_{1}\left(\eta_{1}\right) \hat{g}_{2}\left(\eta_{2}\right) \hat{h}_{1}\left(\zeta_{1}\right) \hat{h}_{2}\left(\zeta_{2}\right) e^{2 \pi i(\xi+\eta+\zeta) \cdot x} d \xi d \eta d \zeta \\
= & \left(\int_{\mathbb{R}^{4}} m^{\prime}\left(\xi_{1}, \eta_{1}, \zeta_{1}\right) \hat{f}(\xi) \hat{g}_{1}\left(\eta_{1}\right) \hat{h}_{1}\left(\zeta_{1}\right) e^{2 \pi i\left(\xi_{1}+\eta_{1}+\zeta_{1}\right) \cdot x_{1}} e^{2 \pi i \xi_{2} \cdot x_{2}} d \xi d \eta_{1} d \zeta_{1}\right) \\
& \cdot\left(\int_{\mathbb{R}^{2}} m^{\prime \prime}\left(\eta_{2}, \zeta_{2}\right) \hat{g}_{2}\left(\eta_{2}\right) \hat{h}_{2}\left(\zeta_{2}\right) e^{2 \pi i\left(\eta_{2}+\zeta_{2}\right) \cdot x_{2}} d \eta_{2} d \zeta_{2}\right) \\
= & \left(\int_{\mathbb{R}^{3}} m^{\prime}\left(\xi_{1}, \eta_{1}, \zeta_{1}\right) \mathscr{F}_{1} f\left(\xi_{1}, x_{2}\right) \hat{g}_{1}\left(\eta_{1}\right) \hat{h}_{1}\left(\zeta_{1}\right) e^{2 \pi i\left(\xi_{1}+\eta_{1}+\zeta_{1}\right) \cdot x_{1}} e^{2 \pi i \xi_{2} \cdot x_{2}} d \xi d \eta_{1} d \zeta_{1}\right) \\
& \cdot\left(\int_{\mathbb{R}^{2}} m^{\prime \prime}\left(\eta_{2}, \zeta_{2}\right) \hat{g}_{2}\left(\eta_{2}\right) \hat{h}_{2}\left(\zeta_{2}\right) e^{2 \pi i\left(\eta_{2}+\zeta_{2}\right) \cdot x_{2}} d \eta_{2} d \zeta_{2}\right) \\
=: \quad & T_{1}\left(f\left(\cdot, x_{2}\right), g_{1}(\cdot), h_{1}(\cdot)\right)\left(x_{1}\right) \cdot T_{2}\left(g_{2}, h_{2}\right)\left(x_{2}\right),
\end{aligned}
$$

where $\mathscr{F}_{1}$ represents the Fourier transform with respect to the first variable. Then its $L^{r}$ norm can be estimated as

$$
\left\|T_{1}\left(f\left(\cdot, x_{2}\right), g_{1}(\cdot), h_{1}(\cdot)\right)\left(x_{1}\right) \cdot T_{2}\left(g_{2}, h_{2}\right)\left(x_{2}\right)\right\|_{L^{r}}^{r}
$$




$$
\begin{aligned}
& =\int\left|T_{1}\left(f\left(\cdot, x_{2}\right), g_{1}(\cdot), h_{1}(\cdot)\right)\left(x_{1}\right)\right|^{r}\left|T_{2}\left(g_{2}, h_{2}\right)\left(x_{2}\right)\right|^{r} d x_{1} d x_{2} \\
& =\int\left(\int\left|T_{1}\left(f\left(\cdot, x_{2}\right), g_{1}(\cdot), h_{1}(\cdot)\right)\left(x_{1}\right)\right|^{r} d x_{1}\right)\left|T_{2}\left(g_{2}, h_{2}\right)\left(x_{2}\right)\right|^{r} d x_{2} \\
& \lesssim \int\left\|f\left(\cdot, x_{2}\right)\right\|_{L_{x_{1}}^{p}}^{r}\left\|g_{1}\right\|_{L_{x_{1}}^{p_{2}}}^{r}\left\|h_{1}\right\|_{L_{x_{1}}^{p_{3}}}^{r}\left|T_{2}\left(g_{2}, h_{2}\right)\left(x_{2}\right)\right|^{r} d x_{2} \\
& =\int\left\|f\left(\cdot, x_{2}\right)\right\|_{L_{x_{1}}^{p}}^{r}\left|T_{2}\left(g_{2}, h_{2}\right)\left(x_{2}\right)\right|^{r} d x_{2} \cdot\left\|g_{1}\right\|_{L_{x_{1}}^{p_{2}}}^{r}\left\|h_{1}\right\|_{L_{x_{1}}^{p_{3}}}^{r} \\
& \lesssim\left(\int\left\|f\left(\cdot, x_{2}\right)\right\|_{L_{x_{1}}^{p}}^{p} d x_{2}\right)^{\frac{r}{p}}\left(\int\left|T_{2}\left(g_{2}, h_{2}\right)\left(x_{2}\right)\right|^{s} d x_{2}\right)^{\frac{r}{s}} \cdot\left\|g_{1}\right\|_{L_{x_{1}}^{p_{2}}}^{r}\left\|h_{1}\right\|_{L_{x_{1}}^{p_{3}}}^{r} \\
& \lesssim\|f\|_{L^{p}}^{r}\left\|g_{2}\right\|_{L_{x_{2}}^{q_{2}}}^{r}\left\|h_{2}\right\|_{L_{x_{2}}^{q_{3}}}^{r}\left\|g_{1}\right\|_{L_{x_{1}}^{p_{2}}}^{r}\left\|h_{1}\right\|_{L_{x_{1}}^{p_{3}}}^{r},
\end{aligned}
$$

where $1<p, p_{2}, p_{3}, q_{2}, q_{3}<\infty, \frac{1}{p_{2}}+\frac{1}{p_{3}}=\frac{1}{q_{2}}+\frac{1}{q_{3}}=\frac{1}{s}$, and we just use the Hölder's inequality, the $L^{r}$ boundedness of the classical one-parameter trilinear Fourier multiplier $T_{1}$ and bilinear multiplier $T_{2}$, i.e.,

$$
\left\|T_{1}\left(f_{1}, f_{2}, f_{3}\right)\right\|_{L^{r}} \lesssim\left\|f_{1}\right\|_{L^{p}}\left\|g_{1}\right\|_{L^{p_{2}}}\left\|h_{1}\right\|_{L^{p_{3}}}, \quad\left\|T_{2}\left(g_{2}, h_{2}\right)\right\|_{L^{s}} \lesssim\left\|g_{2}\right\|_{L^{q_{2}}}\left\|h_{2}\right\|_{L^{q_{3}}} .
$$

Then one can get the same mixed $L^{r}$ estimates for (1.7) under the same tensor product assumption.

Proposition B.2. Let $g(x)=g_{1}\left(x_{1}\right) \otimes g_{2}\left(x_{2}\right), h(x)=h_{1}\left(x_{1}\right) \otimes h_{2}\left(x_{2}\right)$, and $\frac{1}{p}+$ $\frac{1}{p_{2}}+\frac{1}{p_{3}}=\frac{1}{p}+\frac{1}{q_{2}}+\frac{1}{q_{3}}=\frac{1}{r}$. Then (1.7) maps $L^{p} \times L_{x_{1}}^{p_{2}}\left(L_{x_{2}}^{q_{2}}\right) \times L_{x_{1}}^{p_{3}}\left(L_{x_{2}}^{q_{3}}\right) \rightarrow L^{r}$, with $1<p, p_{2}, p_{3}, q_{2}, q_{3}<\infty$ and $0<r<\infty$.

Proof. Recall in our earlier reduction in Section 4, the study of the multipliers (1.7) can be reduced to essentially two types of multipliers, i.e., the classical bi-parameter trilinear multipliers (under the tensor product setting)

$T_{3}\left(f, g_{1} \otimes g_{2}, h_{1} \otimes h_{2}\right)=\int_{\mathbb{R}^{6}} m(\xi, \eta, \zeta) \hat{f}(\xi) \hat{g}_{1}\left(\eta_{1}\right) \hat{g}_{2}\left(\eta_{2}\right) \hat{h}_{1}\left(\zeta_{1}\right) \hat{h}_{2}\left(\zeta_{2}\right) e^{2 \pi i(\xi+\eta+\zeta) \cdot x} d \xi d \eta d \zeta$,

and the ones like (1.8), i.e., (1.8) or

$$
\int_{\mathbb{R}^{6}} m^{\prime}\left(\xi_{2}, \eta_{2}, \zeta_{2}\right) m^{\prime \prime}\left(\eta_{1}, \zeta_{1}\right) \hat{f}(\xi) \hat{g}_{1}\left(\eta_{1}\right) \hat{g}_{2}\left(\eta_{2}\right) \hat{h}_{1}\left(\zeta_{1}\right) \hat{h}_{2}\left(\zeta_{2}\right) e^{2 \pi i(\xi+\eta+\zeta) \cdot x} d \xi d \eta d \zeta
$$

Note that the same argument for (1.8) shows (B.2) maps $L^{p} \times L_{x_{1}}^{p_{2}}\left(L_{x_{2}}^{q_{2}}\right) \times L_{x_{1}}^{p_{3}}\left(L_{x_{2}}^{q_{3}}\right) \rightarrow$ $L^{r}$, with $1<p, p_{2}, p_{3}, q_{2}, q_{3}<\infty$ and $0<r<\infty$.

Then the proof can be completed as long as we can show the mixed norm estimate for $T_{3}$.

Proposition B.3. The bi-parameter trilinear multiplier $T_{3}$ maps $L^{p} \times L_{x_{1}}^{p_{2}}\left(L_{x_{2}}^{q_{2}}\right) \times$ $L_{x_{1}}^{p_{3}}\left(L_{x_{2}}^{q_{3}}\right) \rightarrow L^{r}$, for $1<p, q_{1}, p_{2}, q_{2}, p_{3}, q_{3}<\infty$ and $0<r<\infty$, with $1 / r=$ 
$1 / p+1 / p_{2}+1 / p_{3}=1 / p+1 / q_{2}+1 / q_{3}$, if one assumes $g\left(x_{1}, x_{2}\right)=g\left(x_{1}\right) \otimes g\left(x_{2}\right)$ and $h\left(x_{1}, x_{2}\right)=h\left(x_{1}\right) \otimes h\left(x_{2}\right)$.

For a quick and direct way to see the proof, one can refer to the work [3], where the classical Hörmander type $L^{r}$ estimate was obtained for the multilinear and multiparameter multipliers with limited smoothness. The main idea was to control $m$ by using the Sobolev norm, and the main technique was to take care of the functions, i.e., $f, g, h, \ldots$, by strong maximal functions and Hölder's inequalities. One can see, such methods would go through if one assumes the tensor product form for these functions.

Definition 6. For $f \in \mathcal{S}^{\prime}\left(\mathbb{R}^{6}\right)$, define the bi-parameter Sobolev space

$$
\|f\|_{H^{s_{1}, s_{2}}}:=\left\|(I-\Delta)^{s_{1} / 2, s_{2} / 2} f\right\|_{L^{2}}<\infty,
$$

where

$$
\begin{gathered}
(I-\Delta)^{s_{1} / 2, s_{2} / 2} f= \\
\mathcal{F}^{-1}\left[\left(1+\left|\xi_{1}\right|^{2}+\left|\eta_{1}\right|^{2}+\left|\zeta_{1}\right|^{2}\right)^{s_{1} / 2}\left(1+\left|\xi_{2}\right|^{2}+\left|\eta_{2}\right|^{2}+\left|\zeta_{2}\right|^{2}\right)^{s_{2} / 2} \hat{f}\left(\xi_{1}, \xi_{2}, \eta_{1}, \eta_{2}, \zeta_{1}, \zeta_{2}\right)\right]
\end{gathered}
$$

for $\xi_{1}, \xi_{2}, \eta_{1}, \eta_{2}, \zeta_{1}, \zeta_{2} \in \mathbb{R}$.

Let $m_{j, k}\left(\xi_{1}, \xi_{2}, \eta_{1}, \eta_{2}, \zeta_{1}, \zeta_{2}\right)=m\left(2^{j} \xi_{1}, 2^{k} \xi_{2}, 2^{j} \eta_{1}, 2^{k} \eta_{2}, 2^{j} \zeta_{1}, 2^{k} \zeta_{2}\right) \psi_{1}\left(\xi_{1}, \eta_{1}, \zeta_{1}\right) \psi_{2}\left(\xi_{2}, \eta_{2}, \zeta_{2}\right)$, where smooth cutoff functions $\psi_{1}, \psi_{2}$ satisfy

$$
\operatorname{supp} \psi_{1}, \operatorname{supp} \psi_{2} \subset\left\{u \in \mathbb{R}^{3}: 1 / 2 \leq|u| \leq 2\right\} .
$$

Note that if $m \in \mathcal{B M}\left(\mathbb{R}^{6}\right)$ satisfying

$$
\left|\partial_{\xi_{1}, \xi_{2}}^{\alpha_{1}, \alpha_{2}} \partial_{\eta_{1}, \eta_{2}}^{\beta_{1}, \beta_{2}} \partial_{\zeta_{1}, \zeta_{2}}^{\gamma_{1}, \gamma_{2}} m(\xi, \eta, \zeta)\right| \lesssim \prod_{i=1}^{2} \frac{1}{\left(\left|\xi_{i}\right|+\left|\eta_{i}\right|+\left|\zeta_{i}\right|\right)^{\alpha_{i}+\beta_{i}+\gamma_{i}}},
$$

for $\alpha_{i}+\beta_{i}+\zeta_{i} \leq N_{i}(i=1,2)$, then there holds

$$
\sup _{j, k}\left\|m_{j, k}\right\|_{H^{N_{1}, N_{2}}}<\infty .
$$

We also give a lemma which will be useful later.

Lemma B.1 ( [3]). For any $\epsilon_{1}, \epsilon_{2}>0$, there exists a constant $C>0$ such that

$$
\sup _{r_{1}, r_{2}>0}\left(r_{1}^{n} r_{2}^{n} \int_{\mathbb{R}^{2}} \frac{f(u, v)}{\left(1+r_{1}\left|x_{1}-u\right|\right)^{1+\epsilon_{1}}\left(1+r_{2}\left|x_{2}-v\right|\right)^{1+\epsilon_{2}}} d u d v\right) \leq C M_{s} f\left(x_{1}, x_{2}\right),
$$

where $M_{s}$ is the strong maximal operator.

Now we give a proof for Proposition B.3. 
Proof. We first repeat some arguments in [3]. Consider a decomposition of the symbol, according to the size of the support of each variable. More precisely, take $\phi_{1} \in C^{\infty}$ on $[0, \infty)$ satisfying

$$
\phi_{1}(t)=1 \text { on }[0,1 / 12], \quad \operatorname{supp} \phi_{1} \subset[0,1 / 6],
$$

and also let $\phi_{2}(t)=1-\phi_{1}(t)$. Note that we can write

$$
\begin{aligned}
1\left(\xi_{1}, \eta_{1}, \zeta_{1}\right)= & {\left[\phi_{1}\left(\left|\xi_{1}\right| /\left|\left(\xi_{1}, \eta_{1}, \zeta_{1}\right)\right|\right)+\phi_{2}\left(\left|\xi_{1}\right| /\left|\left(\xi_{1}, \eta_{1}, \zeta_{1}\right)\right|\right)\right] } \\
& \cdot\left[\phi_{1}\left(\left|\eta_{1}\right| /\left|\left(\xi_{1}, \eta_{1}, \zeta_{1}\right)\right|\right)+\phi_{2}\left(\left|\eta_{1}\right| /\left|\left(\xi_{1}, \eta_{1}, \zeta_{1}\right)\right|\right)\right] \\
& \cdot\left[\phi_{1}\left(\left|\zeta_{1}\right| /\left|\left(\xi_{1}, \eta_{1}, \zeta_{1}\right)\right|\right)+\phi_{2}\left(\left|\zeta_{1}\right| /\left|\left(\xi_{1}, \eta_{1}, \zeta_{1}\right)\right|\right)\right] .
\end{aligned}
$$

Obviously a similar expression holds for $1\left(\xi_{2}, \eta_{2}, \zeta_{2}\right)$. Correspondingly the symbol $m$ can be decomposed as

$$
\begin{aligned}
& m\left(\xi_{1}, \xi_{2}, \eta_{1}, \eta_{2}, \zeta_{1}, \zeta_{2}\right) \\
= & m\left(\xi_{1}, \xi_{2}, \eta_{1}, \eta_{2}, \zeta_{1}, \zeta_{2}\right) \cdot 1\left(\xi_{1}, \eta_{1}, \zeta_{1}\right) \cdot 1\left(\xi_{2}, \eta_{2}, \zeta_{2}\right)
\end{aligned}
$$

We group the situations after the decomposition as follows.

For $1\left(\xi_{1}, \eta_{1}, \zeta_{1}\right)$, we consider two groups.

Group $I_{1}$ : The largest component is much larger than the other two, i.e.,

(a) $\left|\xi_{1}\right| \gg\left|\eta_{1}\right|+\left|\zeta_{1}\right|$.

(b) $\left|\eta_{1}\right| \gg\left|\xi_{1}\right|+\left|\zeta_{1}\right|$.

(c) $\left|\zeta_{1}\right| \gg\left|\xi_{1}\right|+\left|\eta_{1}\right|$.

Group $I I_{1}$ : The largest component is comparable to the second largest, i.e.,

(a) $\left|\xi_{1}\right| \approx\left|\eta_{1}\right| \approx\left|\xi_{1}\right|+\left|\eta_{1}\right|+\left|\zeta_{1}\right|$.

(b) $\left|\eta_{1}\right| \approx\left|\zeta_{1}\right| \approx\left|\xi_{1}\right|+\left|\eta_{1}\right|+\left|\zeta_{1}\right|$.

(c) $\left|\zeta_{1}\right| \approx\left|\xi_{1}\right| \approx\left|\xi_{1}\right|+\left|\eta_{1}\right|+\left|\zeta_{1}\right|$.

Similarly, for $1\left(\xi_{2}, \eta_{2}, \zeta_{2}\right)$, we consider two groups.

Group $I_{2}$ : The largest component is much larger than the other two, i.e.,

(a) $\left|\xi_{2}\right| \gg\left|\eta_{2}\right|+\left|\zeta_{2}\right|$.

(b) $\left|\eta_{2}\right| \gg\left|\xi_{2}\right|+\left|\zeta_{2}\right|$.

(c) $\left|\zeta_{2}\right| \gg\left|\xi_{2}\right|+\left|\eta_{2}\right|$.

Group $I I_{2}$ : The largest component is comparable to the second largest, i.e.,

(a) $\left|\xi_{2}\right| \approx\left|\eta_{2}\right| \approx\left|\xi_{2}\right|+\left|\eta_{2}\right|+\left|\zeta_{2}\right|$.

(b) $\left|\eta_{2}\right| \approx\left|\zeta_{2}\right| \approx\left|\xi_{2}\right|+\left|\eta_{2}\right|+\left|\zeta_{2}\right|$.

(c) $\left|\zeta_{2}\right| \approx\left|\xi_{2}\right| \approx\left|\xi_{2}\right|+\left|\eta_{2}\right|+\left|\zeta_{2}\right|$.

According to the symmetry, it suffices to consider

$I_{1} \otimes I_{2}:=\left\{I_{1}(a) \otimes I_{2}(a), I_{1}(a) \otimes I_{2}(b), I_{1}(b) \otimes I_{2}(b), I_{1}(b) \otimes I_{2}(c), \ldots\right\}$,

$I I_{1} \otimes I I_{2}:=\left\{I I_{1}(a) \otimes I I_{2}(a), I I_{1}(a) \otimes I I_{2}(b), I I_{1}(b) \otimes I I_{2}(b), \ldots\right\}$,

$I_{1} \otimes I I_{2}:=\left\{I_{1}(a) \otimes I I_{2}(a), I_{1}(a) \otimes I I_{2}(b), I_{1}(b) \otimes I I_{2}(a), I_{1}(b) \otimes I I_{2}(b), I_{1}(b) \otimes I I_{2}(c), \ldots\right\}$. 
In the rest of the proof, even though $m$ in different groups would be multiplied by corresponding cutoffs which were constructed at the beginning of the proof, for our convenience we will still use $m$ as the notation, since those cutoff functions do not actually play an important role in our calculation.

We first consider the cases that belong to $I_{1} \otimes I_{2}$. We take $I_{1}(a) \otimes I_{2}(b)$ for example. Consider the Littlewood-Paley characterization,

$$
\left\|T_{3}(f, g, h)\right\|_{L^{r}} \lesssim\left\|\left\{\sum_{j, k}\left|\psi_{1}\left(D / 2^{j}\right) \psi_{2}\left(D / 2^{k}\right) T_{3}(f, g, h)\right|^{2}\right\}^{\frac{1}{2}}\right\|_{L^{r}},
$$

where $\psi_{1}\left(D / 2^{j}\right) \psi_{2}\left(D / 2^{k}\right) T_{3}:=\mathcal{F}^{-1}\left[\psi_{1}\left(u / 2^{j}\right) \psi_{2}\left(v / 2^{k}\right) \mathcal{F}\left(T_{3}\right)(u, v)\right]$. For simplicity, we will just denote it by $\psi_{j}(D) \psi_{k}(D) T_{3}$ in the following work. Note that a subscript $j(k)$ always means that the operation is on the first (second) variable.

$$
\begin{aligned}
A_{j, k}= & \psi_{1}\left(D / 2^{j}\right) \psi_{2}\left(D / 2^{k}\right) T_{3}(f, g, h) \\
= & \int_{\mathbb{R}^{6}} m(\xi, \eta, \zeta) e^{i x_{1}\left(\xi_{1}+\eta_{1}+\zeta_{1}\right)} e^{i x_{2}\left(\xi_{2}+\eta_{2}+\zeta_{2}\right)} \psi_{j}\left(\xi_{1}+\eta_{1}+\zeta_{1}\right) \psi_{k}\left(\xi_{2}+\eta_{2}+\zeta_{2}\right) \\
& \cdot \hat{f}\left(\xi_{1}, \xi_{2}\right) \hat{g}\left(\eta_{1}, \eta_{2}\right) \hat{h}\left(\zeta_{1}, \zeta_{2}\right) d \xi_{1} d \xi_{2} d \eta_{1} d \eta_{2} d \zeta_{1} d \zeta_{2} \\
= & \int_{\mathbb{R}^{6}} m(\xi, \eta, \zeta) e^{i x_{1}\left(\xi_{1}+\eta_{1}+\zeta_{1}\right)} e^{i x_{2}\left(\xi_{2}+\eta_{2}+\zeta_{2}\right)} \psi_{j}\left(\xi_{1}+\eta_{1}+\zeta_{1}\right) \psi_{k}\left(\xi_{2}+\eta_{2}+\zeta_{2}\right) \\
& \tilde{\psi}_{j}\left(\xi_{1}\right) \tilde{\psi}_{k}\left(\eta_{2}\right) \hat{f}\left(\xi_{1}, \xi_{2}\right) \hat{g}\left(\eta_{1}, \eta_{2}\right) \hat{h}\left(\zeta_{1}, \zeta_{2}\right) d \xi_{1} d \xi_{2} d \eta_{1} d \eta_{2} d \zeta_{1} d \zeta_{2} \\
= & 2^{3 j+3 k} \int_{\mathbb{R}^{6}}\left(\mathcal{F}^{-1} m_{j, k}\right)\left(2^{j}\left(x_{1}-y_{1}\right), 2^{k}\left(x_{2}-y_{2}\right), 2^{j}\left(x_{1}-z_{1}\right), 2^{k}\left(x_{2}-z_{2}\right),\right. \\
& \left.2^{j}\left(x_{1}-w_{1}\right), 2^{k}\left(x_{2}-w_{2}\right)\right)\left(\tilde{\psi}_{j}(D) f\right)\left(y_{1}, y_{2}\right)\left(\tilde{\psi}_{k}(D)\right) \\
& g\left(z_{1}, z_{2}\right) h\left(w_{1}, w_{2}\right) d y_{1} d y_{2} d z_{1} d z_{2} d w_{1} d w_{2},
\end{aligned}
$$

where we use the properties that $\left|\xi_{1}\right|+\left|\eta_{1}\right|+\left|\zeta_{1}\right| \approx\left|\xi_{1}\right|,\left|\xi_{2}\right|+\left|\eta_{2}\right|+\left|\zeta_{2}\right| \approx\left|\eta_{2}\right|$ and $\tilde{\psi}$ is a properly chosen "completed" function based on the size of support, i.e., $\psi_{j}\left(\xi_{1}+\eta_{1}+\zeta_{1}\right)=\tilde{\psi}_{j}\left(\xi_{1}\right) \psi_{j}\left(\xi_{1}+\eta_{1}+\zeta_{1}\right)$ and similar for $\tilde{\psi}_{k}$.

Then we can estimate $A_{j, k}$ as

$$
\begin{aligned}
\leq \int_{\mathbb{R}^{6}}^{A_{j, k}} 2^{3 j+3 k}\left(1+\left|2^{j}\left(x_{1}-y_{1}\right)\right|+\left|2^{j}\left(x_{1}-z_{1}\right)\right|+\left|2^{j}\left(x_{1}-w_{1}\right)\right|\right)^{s_{1}} \\
\left(1+\left|2^{k}\left(x_{2}-y_{2}\right)\right|+\left|2^{k}\left(x_{2}-z_{2}\right)\right|+\left|2^{k}\left(x_{2}-w_{2}\right)\right|\right)^{s_{2}} \\
\left(\mathcal{F}^{-1} m_{j, k}\right)\left(2^{j}\left(x_{1}-y_{1}\right), 2^{k}\left(x_{2}-y_{2}\right), 2^{j}\left(x_{1}-z_{1}\right), 2^{k}\left(x_{2}-z_{2}\right),\right. \\
\left.2^{j}\left(x_{1}-w_{1}\right), 2^{k}\left(x_{2}-w_{2}\right)\right) \\
\left(1+\left|2^{j}\left(x_{1}-y_{1}\right)\right|+\left|2^{j}\left(x_{1}-z_{1}\right)\right|+\left|2^{j}\left(x_{1}-w_{1}\right)\right|\right)^{-s_{1}} \\
\left(1+\left|2^{k}\left(x_{2}-y_{2}\right)\right|+\left|2^{k}\left(x_{2}-z_{2}\right)\right|+\left|2^{k}\left(x_{2}-w_{2}\right)\right|\right)^{-s_{2}}
\end{aligned}
$$




$$
\begin{aligned}
&\left(\tilde{\psi}_{j}(D) f\right)\left(y_{1}, y_{2}\right)\left(\tilde{\psi}_{k}(D) g\right)\left(z_{1}, z_{2}\right) h\left(w_{1}, w_{2}\right) d y_{1} d y_{2} d z_{1} d z_{2} d w_{1} d w_{2} \\
& \lesssim\left(\int_{\mathbb{R}^{6}}\left(1+\left|y_{1}\right|+\left|z_{1}\right|+\left|w_{1}\right|\right)^{t^{\prime} s_{1}}\left(1+\left|y_{2}\right|+\left|z_{2}\right|+\left|w_{2}\right|\right)^{t^{\prime} s_{2}}\right. \\
&\left.\left|\left(\mathcal{F}^{-1} m_{j, k}\right)\left(y_{1}, y_{2}, z_{1}, z_{2}, w_{1}, w_{2}\right)\right|^{t^{\prime}} d y d z d w\right)^{1 / t^{\prime}} \\
&\left(\int_{\mathbb{R}^{6}} 2^{3 j+3 k}\left(1+\left|2^{j}\left(x_{1}-y_{1}\right)\right|+\left|2^{j}\left(x_{1}-z_{1}\right)\right|+\left|2^{j}\left(x_{1}-w_{1}\right)\right|\right)^{-t s_{1}}\right. \\
&\left(1+\left|2^{k}\left(x_{2}-y_{2}\right)\right|+\left|2^{k}\left(x_{2}-z_{2}\right)\right|+\left|2^{k}\left(x_{2}-w_{2}\right)\right|\right)^{-t s_{2}} \\
&\left.\left|\left(\tilde{\psi}_{j}(D) f\right)\left(y_{1}, y_{2}\right)\left(\tilde{\psi}_{k}(D) g\right)\left(z_{1}, z_{2}\right) h\left(w_{1}, w_{2}\right)\right|^{t} d y_{1} d y_{2} d z_{1} d z_{2} d w_{1} d w_{2}\right)^{\frac{1}{t}} \\
& \lesssim\left\|m_{j, k}\right\|_{H^{s_{1}, s_{2}}}\left(\int_{\mathbb{R}^{6}} \frac{2^{j+k}\left|\left(\tilde{\psi}_{j}(D) f\right)\left(y_{1}, y_{2}\right)\right|^{t}}{\left(1+\left|2^{j}\left(x_{1}-y_{1}\right)\right|\right)^{t s_{1} / 3}\left(1+\left|2^{k}\left(x_{2}-y_{2}\right)\right|\right)^{t s_{2} / 3}}\right. \frac{\left.2_{k}(D) g\right)\left.\left(z_{1}, z_{2}\right)\right|^{t}}{\left(1+\left|2^{j}\left(x_{1}-z_{1}\right)\right|\right)^{t s_{1} / 3}\left(1+\left|2^{k}\left(x_{2}-z_{2}\right)\right|\right)^{t s_{2} / 3}} \\
&\left.\frac{2^{j+k}\left|h\left(w_{1}, w_{2}\right)\right|^{t}}{\left(1+\left|2^{j}\left(x_{1}-w_{1}\right)\right|\right)^{t s_{1} / 3}\left(1+\left|2^{k}\left(x_{2}-w_{2}\right)\right|\right)^{t s_{2} / 3}} d y d z d w\right)^{\frac{1}{t}} \\
& \lesssim\left\|m_{j, k}\right\|_{H^{s_{1}, s_{2}}}\left(M_{s}\left(\left|\tilde{\psi}_{j}(D) f\right|^{t}\right)\left(x_{1}, x_{2}\right)\right)^{\frac{1}{t}}\left(M_{s}\left(\left|\tilde{\psi}_{k}(D) g\right|^{t}\right)\left(x_{1}, x_{2}\right)\right)^{\frac{1}{t}} \\
&\left(M_{s}\left(|h|^{t}\right)\left(x_{1}, x_{2}\right)\right)^{\frac{1}{t}},
\end{aligned}
$$

where $M_{s}$ appears because Lemma B.1 is used, with $t s_{1} / 3>1, t s_{2} / 3>1$. Here we need $t>1$ so that Hölder's inequality can be applied. Also, we take $t<2$ so that the term $\left\|m_{j, k}\right\|_{H^{s_{1}, s_{2}}}$ can be obtained (see [3] for details), and this restriction is also necessary in the arguments later. In short, we need $\max \left(1,3 / s_{1}, 3 / s_{2}\right)<t<2$.

Then

$$
\begin{aligned}
& \left\|T_{3}(f, g, h)\right\|_{L^{r}} \\
\lesssim & \left\|\left\{\sum_{j, k}\left|\psi_{1}\left(D / 2^{j}\right) \psi_{2}\left(D / 2^{k}\right) T_{3}(f, g, h)\right|^{2}\right\}^{\frac{1}{2}}\right\|_{L^{r}} \\
\lesssim & \sup _{j, k}\left\|m_{j, k}\right\|_{H^{s_{1}, s_{2}}} \|\left[\sum_{j, k}\left(M_{s}\left(\left|\tilde{\psi}_{j}(D) f\right|^{t}\right)\left(x_{1}, x_{2}\right)\right)^{\frac{2}{t}}\right. \\
& \left.\left(M_{s}\left(\left|\tilde{\psi}_{k}(D) g\right|^{t}\right)\left(x_{1}, x_{2}\right)\right)^{\frac{2}{t}} \cdot\left(M_{s}\left(|h|^{t}\right)\left(x_{1}, x_{2}\right)\right)^{\frac{2}{t}}\right]^{\frac{1}{2}} \|_{L^{r}} \\
= & \sup _{j, k}\left\|m_{j, k}\right\|_{H^{s_{1}, s_{2}}} \|\left[\sum_{j}\left(M_{s}\left(\left|\tilde{\psi}_{j}(D) f\right|^{t}\right)\left(x_{1}, x_{2}\right)\right)^{\frac{2}{t}}\right]^{\frac{1}{2}}
\end{aligned}
$$




$$
\left[\sum_{k}\left(M_{s}\left(\left|\tilde{\psi}_{k}(D) g\right|^{t}\right)\left(x_{1}, x_{2}\right)\right)^{\frac{2}{t}}\right]^{\frac{1}{2}} \cdot\left(M_{s}\left(|h|^{t}\right)\left(x_{1}, x_{2}\right)\right)^{\frac{1}{t}} \|_{L^{r}} .
$$

If we introduce the tensor product assumption $g\left(x_{1}, x_{2}\right)=g_{1}\left(x_{1}\right) \otimes g_{2}\left(x_{2}\right)$ and $h\left(x_{1}, x_{2}\right)=h_{1}\left(x_{1}\right) \otimes h_{2}\left(x_{2}\right)$, note that

$$
M_{s}(g)\left(x_{1}, x_{2}\right)=M\left(g_{1}\right)\left(x_{1}\right) M\left(g_{2}\right)\left(x_{2}\right), M_{s}(h)\left(x_{1}, x_{2}\right)=M\left(h_{1}\right)\left(x_{1}\right) M\left(h_{2}\right)\left(x_{2}\right),
$$

where $M$ is the Hardy-Littlewood maximal operator.

Thus, we can write

$$
\begin{aligned}
& \left\|T_{3}(f, g, h)\right\|_{L^{r}} \\
\lesssim & \left\|\left\{\sum_{j, k}\left|\psi_{1}\left(D / 2^{j}\right) \psi_{2}\left(D / 2^{k}\right) T_{3}(f, g, h)\right|^{2}\right\}^{\frac{1}{2}}\right\|_{L^{r}} \\
\lesssim & \sup _{j, k}\left\|m_{j, k}\right\|_{H^{s_{1}, s_{2}}} \|\left[\sum_{j}\left(M_{s}\left(\left|\tilde{\psi}_{j}(D) f\right|^{t}\right)\left(x_{1}, x_{2}\right)\right)^{\frac{2}{t}}\right]^{\frac{1}{2}} \\
& \left(M\left(\left|g_{1}\right|^{t}\right)\left(x_{1}\right)\right)^{\frac{1}{t}}\left[\sum_{k}\left(M\left(\left|\tilde{\psi}_{k}(D) g_{2}\right|^{t}\right)\left(x_{2}\right)\right)^{\frac{2}{t}}\right]^{\frac{1}{2}} \cdot\left(M\left(\left|h_{1}\right|^{t}\right)\left(x_{1}\right)\right)^{\frac{1}{t}}\left(M\left(\left|h_{2}\right|^{t}\right)\left(x_{2}\right)\right)^{\frac{1}{t}} \|_{L^{r}} \\
\lesssim & \sup _{j, k}\left\|m_{j, k}\right\|_{H^{s_{1}, s_{2}}}\left\|\left[\sum_{j}\left(M_{s}\left(\left|\tilde{\psi}_{j}(D) f\right|^{t}\right)\left(x_{1}, x_{2}\right)\right)^{\frac{2}{t}}\right]^{\frac{1}{2}}\right\|_{L^{p}} \\
& \left\|\left(M\left(\left|g_{1}\right|^{t}\right)\left(x_{1}\right)\right)^{\frac{1}{t}}\left[\sum_{k}\left(M\left(\left|\tilde{\psi}_{k}(D) g_{2}\right|^{t}\right)\left(x_{2}\right)\right)^{\frac{2}{t}}\right]^{\frac{1}{2}} \cdot\left(M\left(\left|h_{1}\right|^{t}\right)\left(x_{1}\right)\right)^{\frac{1}{t}}\left(M\left(\left|h_{2}\right|^{t}\right)\left(x_{2}\right)\right)^{\frac{1}{t}}\right\|_{L^{s}},
\end{aligned}
$$

where we just apply the Hölder's inequality and $1 / p+1 / s=1 / r$, i.e., $1 / p_{2}+1 / q_{2}=$ $1 / p_{3}+1 / q_{3}=1 / s$. Then using the tensor product setting, the above can be estimated by

$$
\begin{aligned}
=\sup _{j, k} & \left\|m_{j, k}\right\|_{H^{s_{1}, s_{2}}}\left\|\left[\sum_{j}\left(M_{s}\left(\left|\tilde{\psi}_{j}(D) f\right|^{t}\right)(x)\right)^{\frac{2}{t}}\right]^{\frac{1}{2}}\right\|_{L^{p}} \\
& \left\|\left(M\left(\left|g_{1}\right|^{t}\right)\left(x_{1}\right)\right)^{\frac{1}{t}}\left(M\left(\left|h_{1}\right|^{t}\right)\left(x_{1}\right)\right)^{\frac{1}{t}}\right\|_{L^{s}\left(x_{1}\right)} \\
& \left\|\left[\sum_{k}\left(M\left(\left|\tilde{\psi}_{k}(D) g_{2}\right|^{t}\right)\left(x_{2}\right)\right)^{\frac{2}{t}}\right]^{\frac{1}{2}}\left(M\left(\left|h_{2}\right|^{t}\right)\left(x_{2}\right)\right)^{\frac{1}{t}}\right\|_{L^{s}\left(x_{2}\right)}
\end{aligned}
$$

Again by Hölder's inequality, we have

$$
\lesssim \sup _{j, k}\left\|m_{j, k}\right\|_{H^{s_{1}, s_{2}}}\left\|\left[\sum_{j}\left(M_{s}\left(\left|\tilde{\psi}_{j}(D) f\right|^{t}\right)(x)\right)^{\frac{2}{t}}\right]^{\frac{1}{2}}\right\|_{L^{p}}
$$




$$
\begin{gathered}
\left\|\left(M\left(\left|g_{1}\right|^{t}\right)\left(x_{1}\right)\right)^{\frac{1}{t}}\right\|_{L^{p_{2}\left(x_{1}\right)}}\left\|\left[\sum_{k}\left(M\left(\left|\tilde{\psi}_{k}(D) g_{2}\right|^{t}\right)\left(x_{2}\right)\right)^{\frac{2}{t}}\right]^{\frac{1}{2}}\right\|_{L^{q_{2}\left(x_{2}\right)}} \\
\left\|\left(M\left(\left|h_{1}\right|^{t}\right)\left(x_{1}\right)\right)^{\frac{1}{t}}\right\|_{L^{p_{3}\left(x_{1}\right)}}\left\|\left(M\left(\left|h_{2}\right|^{t}\right)\left(x_{2}\right)\right)^{\frac{1}{t}}\right\|_{L^{q_{3}\left(x_{2}\right)}} \\
=\sup _{j, k}\left\|m_{j, k}\right\|_{H^{s_{1}, s_{2}}}\left\|\left[\sum_{j}\left(M_{s}\left(\left|\tilde{\psi}_{j}(D) f\right|^{t}\right)(x)\right)^{\frac{2}{t}}\right]^{\frac{t}{2}}\right\|_{L^{p / t}}^{1 / t} \\
\left\|M\left(\left|g_{1}\right|^{t}\right)\left(x_{1}\right)\right\|_{L^{p_{2} / t}\left(x_{1}\right)}^{1 / t}\left\|\left[\sum_{k}\left(M\left(\left|\tilde{\psi}_{k}(D) g_{2}\right|^{t}\right)\left(x_{2}\right)\right)^{\frac{2}{t}}\right]^{\frac{t}{2}}\right\|_{L^{q_{2} / t}\left(x_{2}\right)}^{1 / t} \\
\left\|M\left(\left|h_{1}\right|^{t}\right)\left(x_{1}\right)\right\|_{L^{p_{3} / t}\left(x_{1}\right)}^{1 / t}\left\|M\left(\left|h_{2}\right|^{t}\right)\left(x_{2}\right)\right\|_{L^{q_{3} / t}\left(x_{2}\right)}^{1 / t} \\
\lesssim \sup _{j, k}\left\|m_{j, k}\right\|_{H^{s_{1}, s_{2}}}\|f\|_{L^{p}}\left\|g_{1}\right\|_{L^{p_{2}}\left(x_{1}\right)}\left\|g_{2}\right\|_{L^{q_{2}}\left(x_{2}\right)}\left\|h_{1}\right\|_{L^{p_{3}\left(x_{1}\right)}}\left\|h_{2}\right\|_{L^{q_{3}}\left(x_{2}\right)},
\end{gathered}
$$

where we use the vector-valued Fefferman-Stein inequality, $2, p, p_{2}, q_{2}, p_{3}, q_{3}>t$.

That means for $I_{1}(a) \otimes I_{2}(b)$, we have proved the boundedness $L^{p} \times L_{x_{1}}^{p_{2}}\left(L_{x_{2}}^{q_{2}}\right) \times$ $L_{x_{1}}^{p_{3}}\left(L_{x_{2}}^{q_{3}}\right) \rightarrow L^{r}$ for $p, p_{2}, q_{2}, p_{3}, q_{3}>t$ and $\max \left(1,3 / s_{1}, 3 / s_{2}\right)<t<2$. Thus, if one takes $s_{1}, s_{2}$ to be properly large, one can take $t$ to be arbitrarily close to 1 , which means the mixed $L^{r}$ estimate can hold for $1<p, p_{2}, q_{2}, p_{3}, q_{3}<\infty$.

The above arguments, with some modifications, will be used to treat all the cases: $I_{1} \otimes I_{2}, I_{1} \otimes I I_{2}$ and $I I_{1} \otimes I I_{2}$. Here we briefly describe the modifications necessary for just a few of these cases.

We start by considering some of the other subcases in $I_{1} \times I_{2}$. For instance, if one considers $I_{1}(b) \times I_{2}(c)$, then

$$
\begin{aligned}
& A_{j, k}= \psi_{1}\left(D / 2^{j}\right) \psi_{2}\left(D / 2^{k}\right) T_{3}(f, g, h) \\
& \lesssim\left\|m_{j, k}\right\|_{H^{s_{1}, s_{2}}}\left(M_{s}\left(|f|^{t}\right)\left(x_{1}, x_{2}\right)\right)^{\frac{1}{t}}\left(M_{s}\left(\left|\tilde{\psi}_{j}(D) g\right|^{t}\right)\left(x_{1}, x_{2}\right)\right)^{\frac{1}{t}} \\
&\left(M_{s}\left(\left|\tilde{\psi}_{k}(D) h\right|^{t}\right)\left(x_{1}, x_{2}\right)\right)^{\frac{1}{t}} .
\end{aligned}
$$

Then

$$
\begin{aligned}
& \left\|T_{3}(f, g, h)\right\|_{L^{r}} \\
\lesssim & \left\|\left\{\sum_{j, k}\left|\psi_{1}\left(D / 2^{j}\right) \psi_{2}\left(D / 2^{k}\right) T_{3}(f, g, h)\right|^{2}\right\}^{\frac{1}{2}}\right\|_{L^{r}} \\
\lesssim & \sup _{j, k}\left\|m_{j, k}\right\|_{H^{s_{1}, s_{2}}}\left\|\left(M_{s}\left(|f|^{t}\right)\right)^{\frac{1}{t}}\right\|_{L^{p}} \|\left[\sum_{j}\left(M\left(\left|\tilde{\psi}_{j}(D) g_{1}\right|^{t}\right)\left(x_{1}\right)\right)^{\frac{2}{t}}\right]^{\frac{1}{2}}
\end{aligned}
$$




$$
\begin{aligned}
& \left(M\left(\left|g_{2}\right|^{t}\right)\left(x_{2}\right)\right)^{\frac{1}{t}} \cdot\left(M\left(\left|h_{1}\right|^{t}\right)\left(x_{1}\right)\right)^{\frac{1}{t}}\left[\sum_{k}\left(M\left(\left|\tilde{\psi}_{k}(D) h_{2}\right|^{t}\right)\left(x_{2}\right)\right)^{\frac{2}{t}}\right]^{\frac{1}{2}} \|_{L^{s}} \\
\lesssim & \sup _{j, k}\left\|m_{j, k}\right\|_{H^{s_{1}, s_{2}}}\|f\|_{L^{p}}\left\|g_{1}\right\|_{L^{p_{2}\left(x_{1}\right)}}\left\|g_{2}\right\|_{L^{q_{2}\left(x_{2}\right)}}\left\|h_{1}\right\|_{L^{p_{3}\left(x_{1}\right)}}\left\|h_{2}\right\|_{L^{q_{3}\left(x_{2}\right)}} .
\end{aligned}
$$

For the cases in $I I_{1} \times I I_{2}$, we first consider $I I_{1}(a) \times I I_{2}(b)$ for example. One can write

$$
\begin{aligned}
& T_{3}(f, g, h)\left(x_{1}, x_{2}\right) \\
= & \sum_{j, k} \int_{\mathbb{R}^{6}} m(\xi, \eta, \zeta) e^{i x_{1}\left(\xi_{1}+\eta_{1}+\zeta_{1}\right)} e^{i x_{2}\left(\xi_{2}+\eta_{2}+\zeta_{2}\right)} \psi_{j}\left(\xi_{1}\right) \tilde{\psi}_{j}\left(\eta_{1}\right) \psi_{k}\left(\eta_{2}\right) \tilde{\psi}_{k}\left(\zeta_{2}\right) \\
& \cdot \hat{f}\left(\xi_{1}, \xi_{2}\right) \hat{g}\left(\eta_{1}, \eta_{2}\right) \hat{h}\left(\zeta_{1}, \zeta_{2}\right) d \xi_{1} d \xi_{2} d \eta_{1} d \eta_{2} d \zeta_{1} d \zeta_{2} \\
= & \sum_{j, k} 2^{3 j+3 k} \int_{\mathbb{R}^{6}}\left(\mathcal{F}^{-1} m_{j, k}\right)\left(2^{j}\left(x_{1}-y_{1}\right), 2^{k}\left(x_{2}-y_{2}\right), 2^{j}\left(x_{1}-z_{1}\right), 2^{k}\left(x_{2}-z_{2}\right),\right. \\
& \left.2^{j}\left(x_{1}-w_{1}\right), 2^{k}\left(x_{2}-w_{2}\right)\right)\left(\psi_{j}(D) f\right)\left(y_{1}, y_{2}\right)\left(\tilde{\psi}_{j}(D) \psi_{k}(D) g\right)\left(z_{1}, z_{2}\right) \\
& \left(\tilde{\psi}_{k}(D) h\right)\left(w_{1}, w_{2}\right) d y_{1} d y_{2} d z_{1} d z_{2} d w_{1} d w_{2},
\end{aligned}
$$

where we take $\sum_{j} \psi_{j}(u)=1$ for $u \neq 0$, and take $\tilde{\psi}$ a properly chosen "completed" function by using properties that $\left|\xi_{1}\right|+\left|\eta_{1}\right|+\left|\zeta_{1}\right| \approx\left|\xi_{1}\right| \approx\left|\eta_{1}\right|,\left|\xi_{2}\right|+\left|\eta_{2}\right|+\left|\zeta_{2}\right| \approx$ $\left|\eta_{2}\right| \approx\left|\zeta_{2}\right|$. Using the control of Sobolev norm as before,

$$
\begin{aligned}
& \left|T_{3}(f, g, h)\left(x_{1}, x_{2}\right)\right| \\
\lesssim & \sup _{j, k}\left\|m_{j, k}\right\|_{H^{s_{1}, s_{2}}} \sum_{j, k}\left[M_{s}\left(\left|\tilde{\psi}_{j}(D) f\right|^{t}\right)\right]^{\frac{1}{t}}\left[M_{s}\left(\left|\tilde{\psi}_{j}(D) \psi_{k}(D) g\right|^{t}\right)\right]^{\frac{1}{t}}\left[M_{s}\left(\left|\tilde{\psi}_{k}(D) h\right|^{t}\right)\right]^{\frac{1}{t}} \\
\lesssim & \sup _{j, k}\left\|m_{j, k}\right\|_{H^{s_{1}, s_{2}}}\left\{\sum_{j}\left[M_{s}\left(\left|\tilde{\psi}_{j}(D) f\right|^{t}\right)\right]^{\frac{2}{t}}\right\}^{\frac{1}{2}}\left\{\sum_{j, k}\left[M_{s}\left(\left|\tilde{\psi}_{j}(D) \psi_{k}(D) g\right|^{t}\right)\right]^{\frac{2}{t}}\right\}^{\frac{1}{2}} \\
& \left\{\left\{\sum_{k}\left[M_{s}\left(\left|\tilde{\psi}_{k}(D) h\right|^{t}\right)\right]^{\frac{2}{t}}\right\}^{\frac{1}{2}},\right.
\end{aligned}
$$

where the Cauchy-Schwartz inequality is used. Then the Hölder's inequality gives

$$
\begin{aligned}
& \left\|T_{3}(f, g, h)\right\|_{L^{r}} \\
\lesssim & \sup _{j, k}\left\|m_{j, k}\right\|_{H^{s_{1}, s_{2}}}\left\|\left\{\sum_{j}\left[M_{s}\left(\left|\tilde{\psi}_{j}(D) f\right|^{t}\right)\right]^{\frac{2}{t}}\right\}^{\frac{1}{2}}\right\|_{L^{p}}
\end{aligned}
$$


BI-PARAMETER TRILINEAR FOURIER MULTIPLIERS WITH FLAG SYMBOL

$$
\begin{aligned}
& \left\|\left\{\sum_{j, k}\left[M_{s}\left(\left|\tilde{\psi}_{j}(D) \psi_{k}(D) g\right|^{t}\right)\right]^{\frac{2}{t}}\right\}^{\frac{1}{2}}\left\{\sum_{k}\left[M_{s}\left(\left|\tilde{\psi}_{k}(D) h\right|^{t}\right)\right]^{\frac{2}{t}}\right\}^{\frac{1}{2}}\right\|_{L^{s}} \\
& \lesssim \sup _{j, k}\left\|m_{j, k}\right\|_{H^{s_{1}, s_{2}}}\left\|\left\{\sum_{j}\left[M_{s}\left(\left|\tilde{\psi}_{j}(D) f\right|^{t}\right)\right]^{\frac{2}{t}}\right\}^{\frac{1}{2}}\right\|_{L^{p}} \\
& \left\|\left\{\sum_{j}\left[M\left(\left|\tilde{\psi}_{j}(D) g_{1}\right|^{t}\right)\right]^{\frac{2}{t}}\right\}^{\frac{1}{2}}\left[M\left(\left|h_{1}\right|^{t}\right)\right]^{\frac{1}{t}}\right\|_{L^{s}\left(x_{1}\right)} \\
& \left\|\left\{\sum_{k}\left[M\left(\left|\tilde{\psi}_{k}(D) g_{2}\right|^{t}\right)\right]^{\frac{2}{t}}\right\}^{\frac{1}{2}}\left\{\sum_{k}\left[M\left(\left|\tilde{\psi}_{k}(D) h_{2}\right|^{t}\right)\right]^{\frac{2}{t}}\right\}^{\frac{1}{2}}\right\|_{L^{s}\left(x_{2}\right)} \\
& \lesssim \sup _{j, k}\left\|m_{j, k}\right\|_{H^{s_{1}, s_{2}}}\left\|\left\{\sum_{j}\left[M\left(\left|\tilde{\psi}_{j}(D) f\right|^{t}\right)\right]^{\frac{2}{t}}\right\}^{\frac{1}{2}}\right\|_{L^{p}} \\
& \left\|\left\{\sum_{j}\left[M\left(\left|\tilde{\psi}_{j}(D) g_{1}\right|^{t}\right)\right]^{\frac{2}{t}}\right\}^{\frac{1}{2}}\right\|_{L^{p_{2}\left(x_{1}\right)}}\left\|\left[M\left(\left|h_{1}\right|^{t}\right)\right]^{\frac{1}{t}}\right\|_{L^{p_{3}\left(x_{1}\right)}} \\
& \left\|\left\{\sum_{k}\left[M\left(\left|\tilde{\psi}_{k}(D) g_{2}\right|^{t}\right)\right]^{\frac{2}{t}}\right\}^{\frac{1}{2}}\right\|_{L^{q_{2}\left(x_{2}\right)}}\left\|\left\{\sum_{k}\left[M\left(\left|\tilde{\psi}_{k}(D) h_{2}\right|^{t}\right)\right]^{\frac{2}{t}}\right\}^{\frac{1}{2}}\right\|_{L^{q_{3}\left(x_{2}\right)}} . \\
& \lesssim \sup _{j, k}\left\|m_{j, k}\right\|_{H^{s_{1}, s_{2}}}\|f\|_{L^{p}}\left\|g_{1}\right\|_{L^{p_{2}\left(x_{1}\right)}}\left\|g_{2}\right\|_{L^{q_{2}\left(x_{2}\right)}}\left\|h_{1}\right\|_{L^{p_{3}\left(x_{1}\right)}}\left\|h_{2}\right\|_{L^{q_{3}\left(x_{2}\right)}} \text {. }
\end{aligned}
$$

For other cases that belong to $I I_{1} \times I I_{2}$, we consider $I I_{1}(a) \times I I_{2}(c)$. One can obtain

$$
\begin{aligned}
& T_{3}(f, g, h)\left(x_{1}, x_{2}\right) \\
= & \sum_{j, k} \int_{\mathbb{R}^{6}} m(\xi, \eta, \zeta) e^{i x_{1}\left(\xi_{1}+\eta_{1}+\zeta_{1}\right)} e^{i x_{2}\left(\xi_{2}+\eta_{2}+\zeta_{2}\right)} \psi_{j}\left(\xi_{1}\right) \tilde{\psi}_{j}\left(\eta_{1}\right) \psi_{k}\left(\xi_{2}\right) \tilde{\psi}_{k}\left(\zeta_{2}\right) \\
& \cdot \hat{f}\left(\xi_{1}, \xi_{2}\right) \hat{g}\left(\eta_{1}, \eta_{2}\right) \hat{h}\left(\zeta_{1}, \zeta_{2}\right) d \xi_{1} d \xi_{2} d \eta_{1} d \eta_{2} d \zeta_{1} d \zeta_{2} \\
\lesssim & \sup _{j, k}\left\|m_{j, k}\right\|_{H^{s_{1}, s_{2}}} \sum_{j, k}\left[M_{s}\left(\left|\psi_{j}(D) \psi_{k}(D) f\right|^{t}\right)\right]^{\frac{1}{t}}\left[M_{s}\left(\left|\tilde{\psi}_{j}(D) g\right|^{t}\right)\right]^{\frac{1}{t}}\left[M_{s}\left(\left|\tilde{\psi}_{k}(D) h\right|^{t}\right)\right]^{\frac{1}{t}} \\
\lesssim & \sup _{j, k}\left\|m_{j, k}\right\|_{H^{s_{1}, s_{2}}}\left\{\sum_{j, k}\left[M_{s}\left(\left|\psi_{j}(D) \psi_{k}(D) f\right|^{t}\right)\right]^{\frac{2}{t}}\right\}^{\frac{1}{2}}\left\{\sum_{j}\left[M_{s}\left(\left|\tilde{\psi}_{j}(D) g\right|^{t}\right)\right]^{\frac{2}{t}}\right\}^{\frac{1}{2}} \\
& \left\{\left\{\sum_{k}\left[M_{s}\left(\left|\tilde{\psi}_{k}(D) h\right|^{t}\right)\right]^{\frac{2}{t}}\right\}^{\frac{1}{2}} .\right.
\end{aligned}
$$


where we use the properties that $\left|\xi_{1}\right|+\left|\eta_{1}\right|+\left|\zeta_{1}\right| \approx\left|\xi_{1}\right| \approx\left|\eta_{1}\right|,\left|\xi_{2}\right|+\left|\eta_{2}\right|+\left|\zeta_{2}\right| \approx$ $\left|\xi_{2}\right| \approx\left|\zeta_{2}\right|$ and $\tilde{\psi}$ is a proper "completed" function as before. Then as before Hölder's inequality gives

$$
\begin{aligned}
& \left\|T_{3}(f, g, h)\right\|_{L^{r}} \\
& \lesssim \sup _{j, k}\left\|m_{j, k}\right\|_{H^{s_{1}, s_{2}}}\left\|\left\{\sum_{j, k}\left[M_{s}\left(\left|\tilde{\psi}_{j}(D) \psi_{k}(D) f\right|^{t}\right)\right]^{\frac{2}{t}}\right\}^{\frac{1}{2}}\right\|_{L^{p}} \\
& \left\|\left\{\sum_{j}\left[M_{s}\left(\left|\tilde{\psi}_{j}(D) g\right|^{t}\right)\right]^{\frac{2}{t}}\right\}^{\frac{1}{2}}\left\{\sum_{k}\left[M_{s}\left(\left|\tilde{\psi}_{k}(D) h\right|^{t}\right)\right]^{\frac{2}{t}}\right\}^{\frac{1}{2}}\right\|_{L^{s}} \\
& \lesssim \sup _{j, k}\left\|m_{j, k}\right\|_{H^{s_{1}, s_{2}}}\left\|\left\{\sum_{j, k}\left[M\left(\left|\tilde{\psi}_{j}(D) \psi_{k}(D) f\right|^{t}\right)\right]^{\frac{2}{t}}\right\}^{\frac{1}{2}}\right\|_{L^{p}} \\
& \left\|\left\{\sum_{j}\left[M\left(\left|\tilde{\psi}_{j}(D) g_{1}\right|^{t}\right)\right]^{\frac{2}{t}}\right\}^{\frac{1}{2}}\right\|_{L^{p_{2}\left(x_{1}\right)}}\left\|\left[M\left(\left|h_{1}\right|^{t}\right)\right]^{\frac{1}{t}}\right\|_{L^{p_{3}\left(x_{1}\right)}} \\
& \left\|\left[M\left(\left|g_{2}\right|^{t}\right)\right]^{\frac{1}{t}}\right\|_{L^{q_{2}\left(x_{1}\right)}}\left\|\left\{\sum_{k}\left[M\left(\left|\tilde{\psi}_{k}(D) h_{2}\right|^{t}\right)\right]^{\frac{2}{t}}\right\}^{\frac{1}{2}}\right\|_{L^{q_{3}\left(x_{2}\right)}} . \\
& \lesssim \sup _{j, k}\left\|m_{j, k}\right\|_{H^{s_{1}, s_{2}}}\|f\|_{L^{p}}\left\|g_{1}\right\|_{L^{p_{2}\left(x_{1}\right)}}\left\|g_{2}\right\|_{L^{q_{2}\left(x_{2}\right)}}\left\|h_{1}\right\|_{L^{p_{3}\left(x_{1}\right)}}\left\|h_{2}\right\|_{L^{q_{3}\left(x_{2}\right)}} \text {. }
\end{aligned}
$$

Other cases in $I I_{1} \times I I_{2}$ can be treated similarly.

Now consider $I_{1} \times I I_{2}$, specifically $I_{1}(a) \times I I_{2}(c)$.

$$
\left\|T_{3}(f, g, h)\right\|_{L^{r}} \lesssim\left\|\left\{\sum_{j}\left|\psi_{1}\left(D / 2^{j}\right) T_{3}(f, g, h)\right|^{2}\right\}^{\frac{1}{2}}\right\|_{L^{r}} .
$$

Let

$$
\begin{aligned}
B_{j}= & \psi_{1}\left(D / 2^{j}\right) T_{3}(f, g, h)\left(x_{1}, x_{2}\right) \\
= & \int_{\mathbb{R}^{6}} m(\xi, \eta, \zeta) e^{i x_{1}\left(\xi_{1}+\eta_{1}+\zeta_{1}\right)} e^{i x_{2}\left(\xi_{2}+\eta_{2}+\zeta_{2}\right)} \psi_{j}\left(\xi_{1}+\eta_{1}+\zeta_{1}\right) \\
& \cdot \hat{f}\left(\xi_{1}, \xi_{2}\right) \hat{g}\left(\eta_{1}, \eta_{2}\right) \hat{h}\left(\zeta_{1}, \zeta_{2}\right) d \xi_{1} d \xi_{2} d \eta_{1} d \eta_{2} d \zeta_{1} d \zeta_{2} \\
= & \int_{\mathbb{R}^{6}} \sum_{k} m(\xi, \eta, \zeta) e^{i x_{1}\left(\xi_{1}+\eta_{1}+\zeta_{1}\right)} e^{i x_{2}\left(\xi_{2}+\eta_{2}+\zeta_{2}\right)} \psi_{j}\left(\xi_{1}+\eta_{1}+\zeta_{1}\right) \tilde{\psi}_{j}\left(\xi_{1}\right) \\
& \psi_{k}\left(\xi_{2}\right) \tilde{\psi}_{k}\left(\zeta_{2}\right) \hat{f}\left(\xi_{1}, \xi_{2}\right) \hat{g}\left(\eta_{1}, \eta_{2}\right) \hat{h}\left(\zeta_{1}, \zeta_{2}\right) d \xi_{1} d \xi_{2} d \eta_{1} d \eta_{2} d \zeta_{1} d \zeta_{2} \\
= & \sum_{k} 2^{3 j+3 k} \int_{\mathbb{R}^{6}}\left(\mathcal{F}^{-1} m_{j, k}\right)\left(2^{j}\left(x_{1}-y_{1}\right), 2^{k}\left(x_{2}-y_{2}\right), 2^{j}\left(x_{1}-z_{1}\right), 2^{k}\left(x_{2}-z_{2}\right),\right.
\end{aligned}
$$




$$
\left.2^{j}\left(x_{1}-w_{1}\right), 2^{k}\left(x_{2}-w_{2}\right)\right)\left(\tilde{\psi}_{j}(D) \psi_{k}(D) f\right)\left(y_{1}, y_{2}\right) g\left(z_{1}, z_{2}\right)\left(\tilde{\psi}_{k}(D) h\right)\left(w_{1}, w_{2}\right) d y d z d w
$$

where we use the properties that $\left|\xi_{1}\right|+\left|\eta_{1}\right|+\left|\zeta_{1}\right| \approx\left|\xi_{1}\right|,\left|\xi_{2}\right|+\left|\eta_{2}\right|+\left|\zeta_{2}\right| \approx\left|\xi_{2}\right| \approx\left|\zeta_{2}\right|$ and $\tilde{\psi}$ is a properly chosen "completed" function.

Then as before one can get

$$
\begin{gathered}
B_{j} \lesssim \sup _{k}\left\|m_{j, k}\right\|_{H^{s_{1}, s_{2}}} \sum_{k}\left(M_{s}\left(\left|\tilde{\psi}_{j}(D) \psi_{k}(D) f\right|^{t}\right)\left(x_{1}, x_{2}\right)\right)^{\frac{1}{t}}\left(M_{s}\left(|g|^{t}\right)\left(x_{1}, x_{2}\right)\right)^{\frac{1}{t}} \\
\left(M_{s}\left(\left|\tilde{\psi}_{k}(D) h\right|^{t}\right)\left(x_{1}, x_{2}\right)\right)^{\frac{1}{t}} \\
\lesssim \sup _{k}\left\|m_{j, k}\right\|_{H^{s_{1}, s_{2}}}\left\{\sum_{k}\left(M_{s}\left(\left|\tilde{\psi}_{j}(D) \psi_{k}(D) f\right|^{t}\right)\left(x_{1}, x_{2}\right)\right)^{\frac{2}{t}}\right\}^{\frac{1}{2}}\left(M_{s}\left(|g|^{t}\right)\left(x_{1}, x_{2}\right)\right)^{\frac{1}{t}} \\
\left\{\sum_{k}\left(M_{s}\left(\left|\tilde{\psi}_{k}(D) h\right|^{t}\right)\left(x_{1}, x_{2}\right)\right)^{\frac{2}{t}}\right\}^{\frac{1}{2}},
\end{gathered}
$$

where the Cauch-Schwartz inequality is used. Then

$$
\begin{aligned}
& \left\|T_{3}(f, g, h)\right\|_{L^{r}} \\
& \lesssim\left\|\left\{\sum_{j}\left|\psi_{1}\left(D / 2^{j}\right) T_{3}(f, g, h)\right|^{2}\right\}^{\frac{1}{2}}\right\|_{L^{r}} \\
& \lesssim \sup _{j, k}\left\|m_{j, k}\right\|_{H^{s_{1}, s_{2}}} \|\left\{\sum_{j, k}\left(M_{s}\left(\left|\tilde{\psi}_{j}(D) \psi_{k}(D) f\right|^{t}\right)\left(x_{1}, x_{2}\right)\right)^{\frac{2}{t}}\right\}^{\frac{1}{2}} \\
& \left(M_{s}\left(|g|^{t}\right)\left(x_{1}, x_{2}\right)\right)^{\frac{1}{t}} \cdot\left\{\sum_{k}\left(M_{s}\left(\left|\tilde{\psi}_{k}(D) h\right|^{t}\right)\left(x_{1}, x_{2}\right)\right)^{\frac{2}{t}}\right\}^{\frac{1}{2}} \|_{L^{r}} \\
& \lesssim \sup _{j, k}\left\|m_{j, k}\right\|_{H^{s_{1}, s_{2}}}\left\|\left\{\sum_{j, k}\left(M_{s}\left(\left|\tilde{\psi}_{j}(D) \psi_{k}(D) f\right|^{t}\right)\left(x_{1}, x_{2}\right)\right)^{\frac{2}{t}}\right\}^{\frac{1}{2}}\right\|_{L^{p}} \\
& \left\|\left(M_{s}\left(|g|^{t}\right)\left(x_{1}, x_{2}\right)\right)^{\frac{1}{t}} \cdot\left\{\sum_{k}\left(M_{s}\left(\left|\tilde{\psi}_{k}(D) h\right|^{t}\right)\left(x_{1}, x_{2}\right)\right)^{\frac{2}{t}}\right\}^{\frac{1}{2}}\right\|_{L^{s}} \\
& \lesssim \sup _{j, k}\left\|m_{j, k}\right\|_{H^{s_{1}, s_{2}}}\left\|\left\{\sum_{j, k}\left(M_{s}\left(\left|\tilde{\psi}_{j}(D) \psi_{k}(D) f\right|^{t}\right)\left(x_{1}, x_{2}\right)\right)^{\frac{2}{t}}\right\}^{\frac{1}{2}}\right\|_{L^{p}} \\
& \left\|\left(M\left(\left|g_{1}\right|^{t}\right)\left(x_{1}\right)\right)^{\frac{1}{t}} \cdot\left(M\left(\left|h_{1}\right|^{t}\right)\left(x_{1}\right)\right)^{\frac{1}{t}}\right\|_{L^{s}\left(x_{1}\right)}
\end{aligned}
$$




$$
\begin{aligned}
& \left\|\left(M\left(\left|g_{2}\right|^{t}\right)\left(x_{2}\right)\right)^{\frac{1}{t}} \cdot\left\{\sum_{k}\left(M\left(\left|\tilde{\psi}_{k}(D) h_{2}\right|^{t}\right)\left(x_{2}\right)\right)^{\frac{2}{t}}\right\}^{\frac{1}{2}}\right\|_{L^{s}\left(x_{2}\right)} \\
& \lesssim \sup _{j, k}\left\|m_{j, k}\right\|_{H^{s_{1}, s_{2}}}\left\|\left\{\sum_{j, k}\left(M_{s}\left(\left|\tilde{\psi}_{j}(D) \psi_{k}(D) f\right|^{t}\right)\left(x_{1}, x_{2}\right)\right)^{\frac{2}{t}}\right\}^{\frac{1}{2}}\right\|_{L^{p}} \\
& \left\|\left(M\left(\left|g_{1}\right|^{t}\right)\left(x_{1}\right)\right)^{\frac{1}{t}}\right\|_{L^{p_{2}\left(x_{1}\right)}} \cdot\left\|\left(M\left(\left|h_{1}\right|^{t}\right)\left(x_{1}\right)\right)^{\frac{1}{t}}\right\|_{L^{p_{3}\left(x_{1}\right)}} \\
& \left\|\left(M\left(\left|g_{2}\right|^{t}\right)\left(x_{2}\right)\right)^{\frac{1}{t}}\right\|_{L^{q_{2}\left(x_{2}\right)}} \cdot\left\|\left\{\sum_{k}\left(M\left(\left|\tilde{\psi}_{k}(D) h_{2}\right|^{t}\right)\left(x_{2}\right)\right)^{\frac{2}{t}}\right\}^{\frac{1}{2}}\right\|_{L^{q_{3}\left(x_{2}\right)}} \\
& \lesssim \sup _{j, k}\left\|m_{j, k}\right\|_{H^{s_{1}, s_{2}}}\|f\|_{L^{p}}\left\|g_{1}\right\|_{L^{p_{2}\left(x_{1}\right)}}\left\|g_{2}\right\|_{L^{q_{2}\left(x_{2}\right)}}\left\|h_{1}\right\|_{L^{p_{3}\left(x_{1}\right)}}\left\|h_{2}\right\|_{L^{q_{3}\left(x_{2}\right)}} .
\end{aligned}
$$

Similarly, for other cases in $I_{1} \times I I_{2}$, if we take $I_{1}(a) \times I I_{2}(b)$,

$$
\begin{aligned}
B_{j}:= & \psi_{1}\left(D / 2^{j}\right) T_{3}(f, g, h) \\
\lesssim & \sup _{k}\left\|m_{j, k}\right\|_{H^{s_{1}, s_{2}}}\left(M_{s}\left(\left|\tilde{\psi}_{j}(D) f\right|^{t}\right)\left(x_{1}, x_{2}\right)\right)^{\frac{1}{t}}\left\{\sum_{k}\left(M_{s}\left(\left|\psi_{k}(D) g\right|^{t}\right)\left(x_{1}, x_{2}\right)\right)^{\frac{2}{t}}\right\}^{\frac{1}{2}} \\
& \left\{\sum_{k}\left(M_{s}\left(\left|\tilde{\psi}_{k}(D) h\right|^{t}\right)\left(x_{1}, x_{2}\right)\right)^{\frac{2}{t}}\right\}^{\frac{1}{2}} .
\end{aligned}
$$

Then

$$
\begin{aligned}
& \left\|T_{3}(f, g, h)\right\|_{L^{r}} \\
\lesssim & \left\|\left\{\sum_{j}\left|\psi_{1}\left(D / 2^{j}\right) T_{3}(f, g, h)\right|^{2}\right\}^{\frac{1}{2}}\right\|_{L^{r}} \\
\lesssim & \sup _{j, k}\left\|m_{j, k}\right\|_{H^{s_{1}, s_{2}}} \|\left\{\sum_{j}\left(M_{s}\left(\left|\tilde{\psi}_{j}(D)\right|^{t}\right)\left(x_{1}, x_{2}\right)\right)^{\frac{2}{t}}\right\}^{\frac{1}{2}} \\
& \left\{\sum_{k}\left(M_{s}\left(\left|\psi_{k}(D) g\right|^{t}\right)\left(x_{1}, x_{2}\right)\right)^{\frac{2}{t}}\right\}^{\frac{1}{2}} \cdot\left\{\sum_{k}\left(M_{s}\left(\left|\tilde{\psi}_{k}(D) h\right|^{t}\right)\left(x_{1}, x_{2}\right)\right)^{\frac{2}{t}}\right\}^{\frac{1}{2}} \|_{L^{r}} \\
\lesssim & \sup _{j, k}\left\|m_{j, k}\right\|_{H^{s_{1}, s_{2}}}\left\|\left\{\sum_{j}\left(M_{s}\left(\left|\tilde{\psi}_{j}(D) f\right|^{t}\right)\left(x_{1}, x_{2}\right)\right)^{\frac{2}{t}}\right\}^{\frac{1}{2}}\right\|_{L^{p}} \\
& \left\|\left(M\left(\left|g_{1}\right|^{t}\right)\left(x_{1}\right)\right)^{\frac{1}{t}} \cdot\left(M\left(\left|h_{1}\right|^{t}\right)\left(x_{1}\right)\right)^{\frac{1}{t}}\right\| \|_{L^{s}\left(x_{1}\right)}
\end{aligned}
$$




$$
\begin{aligned}
& \left\|\left\{\sum_{k}\left(M\left(\left|\tilde{\psi}_{k}(D) g_{2}\right|^{t}\right)\left(x_{2}\right)\right)^{\frac{2}{t}}\right\}^{\frac{1}{2}} \cdot\left\{\sum_{k}\left(M\left(\left|\tilde{\psi}_{k}(D) h_{2}\right|^{t}\right)\left(x_{2}\right)\right)^{\frac{2}{t}}\right\}^{\frac{1}{2}}\right\|_{L^{s}\left(x_{2}\right)} \\
& \lesssim \sup _{j, k}\left\|m_{j, k}\right\|_{H^{s_{1}, s_{2}}}\|f\|_{L^{p}}\left\|g_{1}\right\|_{L^{p_{2}\left(x_{1}\right)}}\left\|g_{2}\right\|_{L^{q_{2}\left(x_{2}\right)}}\left\|h_{1}\right\|_{L^{p_{3}\left(x_{1}\right)}}\left\|h_{2}\right\|_{L^{q_{3}\left(x_{2}\right)}} .
\end{aligned}
$$

In this way such modification gives the desired mixed norm estimate.

\section{APPENDix C.}

In this section, we sketch how our reduction can be used to establish the weighted mixed norm estimates. Thus, the results of Appendix B are the unweighted estimates which we presented first for clarity.

Recall the Muckenhoupts $A_{p}$ weights.

\section{Definition 7 ( [17]).}

(a) We say a weight $w \geq 0$ belong to the Muckenhoupt class $A_{p}(\mathbb{R})(1<p<\infty)$ if

$$
\sup _{I}\left(\frac{1}{|I|} \int_{I} w(x) d x\right)\left(\frac{1}{|I|} \int_{I} w(x)^{\frac{1}{1-p}} d x\right)^{p-1}<\infty
$$

where the supremum is taken over all intervals in $\mathbb{R}$. Also, $w \geq 0$ belong to $A_{1}$ if there exists some $C>0$ such that

$$
\sup _{I} \frac{1}{|I|} \int_{I} w(x) d x \leq C w(x) .
$$

Then class $A_{\infty}$ is defined to be $A_{\infty}=\cup_{1 \leq p<\infty} A_{p}$.

(b) The weighted $L^{p}$ space is defined via the norm $\|f\|_{L_{w}^{p}(\mathbb{R})}=\left(\int_{\mathbb{R}}|f(x)|^{p} w(x) d x\right)^{\frac{1}{p}}$.

In the product setting, $A_{p}(\mathbb{R} \times \mathbb{R})$ is defined the same way, replacing the intervals $I$ by rectangles. Then we introduce several lemmas that will be useful later. Since they will be true for both $A_{p}(\mathbb{R} \times \mathbb{R})$ and $A_{p}(\mathbb{R})$, for convenience we will simply use the notation $A_{p}$.

Lemma C.1 ( [12]). Let $1<p<\infty$ and $w \in A_{p}$, then

(1) $w^{1-p^{\prime}} \in A_{p^{\prime}}$,

(2) $\exists 1<q<p$ such that $w \in A_{q}$.

Lemma C.2 ( [3]). Let $w_{j} \in A_{p_{j}}$ for $1 \leq j \leq m$ for some $1 \leq p_{1}, \ldots, p_{m} \leq \infty$. Then for any $\theta_{1}+\cdots \theta_{m}=1$ with $0<\theta_{1}, \ldots \theta_{m}<1$, there holds

$$
w_{1}^{\theta_{1}} \cdots w_{m}^{\theta_{m}} \in A_{\max \left(p_{1}, \ldots, p_{m}\right)} .
$$


Lemma C.3 ( [9]). Let $1<p, q<\infty$ and $w \in A_{p}$, the weighted vector-valued maximal inequality

$$
\left\|\left\{\sum_{j \in \mathbb{Z}}\left(M_{s} f_{j}\right)^{q}\right\}^{\frac{1}{q}}\right\|_{L^{p}(w)} \lesssim\left\|\left\{\sum_{j \in \mathbb{Z}}\left(f_{j}\right)^{q}\right\}^{\frac{1}{q}}\right\|_{L^{p}(w)},
$$

for all sequences $\left\{f_{j}\right\}_{j \in \mathbb{Z}}$ of locally integrable functions on $\mathbb{R} \times \mathbb{R}$.

Lemma C.4 ( [10]). Let $1<p<\infty$ and $w \in A_{p}$. Suppose $\psi_{i} \in \mathcal{S}(\mathbb{R})$ satisfies supp $\hat{\psi}_{i} \subseteq\left\{u \in \mathbb{R}: 1 / a_{i} \leq|u| \leq a_{i}\right\}$ for $a_{i}>1(i=1,2)$, then there holds

$$
\left\|\left\{\sum_{j, k \in \mathbb{Z}}\left|\psi_{1}\left(D / 2^{j}\right) \psi_{2}\left(D / 2^{k}\right) f\right|^{2}\right\}^{\frac{1}{2}}\right\|_{L^{p}(w)} \lesssim\|f\|_{L^{p}(w)} \text { for } f \in L^{p}(w) .
$$

Moreover, if $\sum_{k} \hat{\psi}_{i}\left(u / 2^{k}\right)=1$ for $u \neq 0(i=1,2)$, then

$$
\left\|\left\{\sum_{j, k \in \mathbb{Z}}\left|\psi_{1}\left(D / 2^{j}\right) \psi_{2}\left(D / 2^{k}\right) f\right|^{2}\right\}^{\frac{1}{2}}\right\|_{L^{p}(w)} \approx\|f\|_{L^{p}(w)} \text { for } f \in L^{p}(w) .
$$

Lemma C.5 ( [24]). Let $0<p<\infty$ and $w \in A_{\infty}$ and $\psi_{1}, \psi_{2}$ be as in the previous lemma. Then for a locally integrable function $f \in H^{p}(w)$, there holds

$$
\|f\|_{L^{p}(w)} \lesssim\left\|\left\{\sum_{j, k \in \mathbb{Z}}\left|\psi_{1}\left(D / 2^{j}\right) \psi_{2}\left(D / 2^{k}\right) f\right|^{2}\right\}^{\frac{1}{2}}\right\|_{L^{p}(w)} .
$$

In order to establish the weighted mixed norm estimate for (1.7) under the tensor product assumption $g_{1} \otimes g_{2}$ and $h_{1} \otimes h_{2}$, which is stated as Theorem [1.9, as before it suffices to prove the same boundedness property for our reduced operator (1.8) (as well as (B.2) by symmetry), and the bi-parameter trilinear multiplier $T_{3}$ defined in (B.1).

We first study the weighted estimate for the reduced operator (1.8). One will see, for technical purposes, we will first consider the Sobolev regularity for the symbols instead of the Hörmander type condition.

Proposition C.1. Let $g(x)=g_{1}\left(x_{1}\right) \otimes g_{2}\left(x_{2}\right), h(x)=h_{1}\left(x_{1}\right) \otimes h_{2}\left(x_{2}\right)$, and the multipliers in (1.8) satisfy the limited smoothness condition in the sense that for $3 / 2<s_{1} \leq 3,1<s_{2} \leq 2$,

$$
\sup _{j \in \mathbb{Z}}\left\|m_{j}^{\prime}\right\|_{H^{s_{1}}}<\infty, \quad \sup _{k \in \mathbb{Z}}\left\|m_{k}^{\prime \prime}\right\|_{H^{s_{2}}}<\infty .
$$

Assume that

$$
\min \left(p, p_{2}, p_{3}\right)>3 / s_{1} \quad \text { and } \quad w_{1}^{1}\left(x_{1}\right) \in A_{p s_{1} / 3}, w_{1}^{2}\left(x_{1}\right) \in A_{p_{2} s_{1} / 3}, w_{1}^{3}\left(x_{1}\right) \in A_{p_{3} s_{1} / 3},
$$




$$
\min \left(q_{2}, q_{3}\right)>2 / s_{2} \quad \text { and } \quad w_{2}^{1}\left(x_{2}\right) \in A_{\infty}, w_{2}^{2}\left(x_{2}\right) \in A_{q_{2} s_{2} / 2}, w_{2}^{3}\left(x_{2}\right) \in A_{q_{3} s_{2} / 2},
$$

then (1.8) maps $L^{p}\left(w_{1}^{1} \otimes w_{2}^{1}\right) \times L_{x_{1}}^{p_{2}}\left(w_{1}^{2}\right)\left(L_{x_{2}}^{q_{2}}\left(w_{2}^{2}\right)\right) \times L_{x_{1}}^{p_{3}}\left(w_{1}^{3}\right)\left(L_{x_{2}}^{q_{3}}\left(w_{2}^{3}\right)\right) \rightarrow L^{r}\left(w_{1} \otimes w_{2}\right)$ for $\frac{1}{p}+\frac{1}{p_{2}}+\frac{1}{p_{3}}=\frac{1}{p}+\frac{1}{q_{2}}+\frac{1}{q_{3}}=\frac{1}{r}$ with $0<r<\infty, 1<p, p_{2}, p_{3}, q_{2}, q_{3}<\infty$, where

$$
\begin{aligned}
& w_{1}\left(x_{1}\right)=\left(w_{1}^{1}\right)^{r / p} \cdot\left(w_{1}^{2}\right)^{r / p_{2}} \cdot\left(w_{1}^{3}\right)^{r / p_{3}} \\
& w_{2}\left(x_{2}\right)=\left(w_{2}^{1}\right)^{r / p} \cdot\left(w_{2}^{2}\right)^{r / q_{2}} \cdot\left(w_{2}^{3}\right)^{r / q_{3}} .
\end{aligned}
$$

In particular, by taking $w_{1}^{1}=w_{1}^{2}=w_{1}^{3}=w_{1} \in A_{\min \left(p s_{1} / 3, p_{2} s_{1} / 3, p_{3} s_{1} / 3\right)}, w_{2}^{1}=$ $w_{2}^{2}=w_{2}^{3}=w_{2} \in A_{\min \left(q_{2} s_{2} / 2, q_{3} s_{2} / 2\right)}$, (1.8) $\operatorname{maps} L^{p}\left(w_{1} \otimes w_{2}\right) \times L_{x_{1}}^{p_{2}}\left(w_{1}\right)\left(L_{x_{2}}^{q_{2}}\left(w_{2}\right)\right) \times$ $L_{x_{1}}^{p_{3}}\left(w_{1}\right)\left(L_{x_{2}}^{q_{3}}\left(w_{2}\right)\right) \rightarrow L^{r}\left(w_{1} \otimes w_{2}\right)$.

Proof. As in the proof Proposition B.1, we just need to apply an iteration argument and use the single-parameter weighted estimate.

$$
\begin{aligned}
& \left\|T_{1}\left(f\left(\cdot, x_{2}\right), g_{1}(\cdot), h_{1}(\cdot)\right)\left(x_{1}\right) \cdot T_{2}\left(g_{2}, h_{2}\right)\left(x_{2}\right)\right\|_{L^{r}\left(w_{1} \otimes w_{2}\right)}^{r} \\
& =\int\left|T_{1}\left(f\left(\cdot, x_{2}\right), g_{1}(\cdot), h_{1}(\cdot)\right)\left(x_{1}\right)\right|^{r} w_{1}\left(x_{1}\right)\left|T_{2}\left(g_{2}, h_{2}\right)\left(x_{2}\right)\right|^{r} w_{2}\left(x_{2}\right) d x_{1} d x_{2} \\
& =\int\left(\int\left|T_{1}\left(f\left(\cdot, x_{2}\right), g_{1}(\cdot), h_{1}(\cdot)\right)\left(x_{1}\right)\right|^{r} w_{1}\left(x_{1}\right) d x_{1}\right)\left|T_{2}\left(g_{2}, h_{2}\right)\left(x_{2}\right)\right|^{r} w_{2}(x) d x_{2} \\
& \lesssim \int\left\|f\left(\cdot, x_{2}\right)\right\|_{L_{x_{1}}^{p}\left(w_{1}^{1}\right)}^{r}\left\|g_{1}\right\|_{L_{x_{1}}^{p_{2}\left(w_{1}^{2}\right)}}^{r}\left\|h_{1}\right\|_{L_{x_{1}}^{p_{3}}\left(w_{1}^{3}\right)}^{r}\left|T_{2}\left(g_{2}, h_{2}\right)\left(x_{2}\right)\right|^{r} w_{2}\left(x_{2}\right) d x_{2} \\
& =\int\left\|f\left(\cdot, x_{2}\right)\right\|_{L_{x_{1}}^{p}\left(w_{1}^{1}\right)}^{r}\left|T_{2}\left(g_{2}, h_{2}\right)\left(x_{2}\right)\right|^{r} w_{2}\left(x_{2}\right) d x_{2} \cdot\left\|g_{1}\right\|_{L_{x_{1}}^{p_{2}\left(w_{1}^{2}\right)}}^{r}\left\|h_{1}\right\|_{L_{x_{1}}^{p_{3}}\left(w_{1}^{3}\right)}^{r} \\
& \lesssim\left(\int\left\|f\left(\cdot, x_{2}\right)\right\|_{L_{x_{1}}^{p}\left(w_{1}^{1}\right)}^{p} w_{2}^{1}\left(x_{2}\right) d x_{2}\right)^{\frac{r}{p}}\left(\int\left|T_{2}\left(g_{2}, h_{2}\right)\left(x_{2}\right)\right|^{s_{0}}\left(w_{2}^{2}\right)^{s_{0} / q_{2}} \cdot\left(w_{2}^{3}\right)^{s_{0} / q_{3}} d x_{2}\right)^{\frac{r}{s_{0}}} \\
& \cdot\left\|g_{1}\right\|_{L_{x_{1}}^{p_{2}}}^{r}\left\|h_{1}\right\|_{L_{x_{1}}^{p_{3}}}^{r} \\
& \lesssim\|f\|_{L^{p}\left(w_{1}^{1} \otimes w_{2}^{1}\right)}^{r}\left\|g_{2}\right\|_{L_{x_{2}}^{q_{2}\left(w_{2}^{2}\right)}}^{r}\left\|h_{2}\right\|_{L_{x_{2}}^{q_{3}\left(w_{2}^{3}\right)}}^{r}\left\|g_{1}\right\|_{L_{x_{1}}^{p_{2}\left(w_{1}^{2}\right)}}^{r}\left\|h_{1}\right\|_{L_{x_{1}}^{p_{3}\left(w_{1}^{3}\right)}}^{r},
\end{aligned}
$$

where $1<p, p_{2}, p_{3}, q_{2}, q_{3}<\infty, \frac{1}{p_{2}}+\frac{1}{p_{3}}=\frac{1}{q_{2}}+\frac{1}{q_{3}}=\frac{1}{s_{0}}$, and we just use the Hölder's inequality, the weighted norm estimate of the classical one-parameter trilinear Fourier multiplier $T_{1}$ and bilinear multiplier $T_{2}$, i.e., under condition (C.1) there holds

$$
\left\|T_{1}\left(f_{1}, f_{2}, f_{3}\right)\right\|_{L^{r}\left(w_{1}\right)} \lesssim\left\|f_{1}\right\|_{L^{p}\left(w_{1}^{1}\right)}\left\|g_{1}\right\|_{L^{p_{2}\left(w_{1}^{2}\right)}}\left\|h_{1}\right\|_{L^{p_{3}\left(w_{1}^{3}\right)}}
$$

and under condition (C.2) there holds

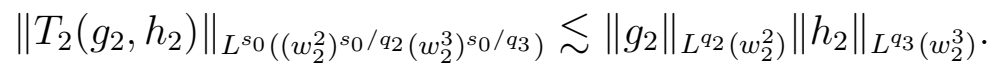

Such single-parameter estimates can be found, e.g., in [3, 11]. 
We note that weighted estimates for the classical bi-parameter and trilinear Fourier multipliers were established in [3]. For the weighted mixed norm estimate for the bi-parameter and trilinear operator $T_{3}$, we have the following

Proposition C.2. Let $g(x)=g_{1}\left(x_{1}\right) \otimes g_{2}\left(x_{2}\right), h(x)=h_{1}\left(x_{1}\right) \otimes h_{2}\left(x_{2}\right)$ and the symbol in $T_{3}$ satisfy the limited smoothness condition in the sense that for $3 / 2<s \leq 3$

$$
\sup _{j, k \in \mathbb{Z}}\left\|m_{j, k}\right\|_{H^{s, s}}<\infty \text {. }
$$

Assume that

$$
\begin{aligned}
& \min \left(p, p_{2}, p_{3}\right)>3 / s \quad \text { and } \quad w_{1}^{1}\left(x_{1}\right) \in A_{p s / 3}, w_{1}^{2}\left(x_{1}\right) \in A_{p_{2} s / 3}, w_{1}^{3}\left(x_{1}\right) \in A_{p_{3} / 3}, \\
& \min \left(p, q_{2}, q_{3}\right)>3 / s \quad \text { and } \quad w_{2}^{1}\left(x_{2}\right) \in A_{p s / 3}, w_{2}^{2}\left(x_{2}\right) \in A_{q_{2} s / 3}, w_{2}^{3}\left(x_{2}\right) \in A_{q_{3} s / 3} \text {, }
\end{aligned}
$$

then $T_{3}$ maps $L^{p}\left(w_{1}^{1} \otimes w_{2}^{1}\right) \times L_{x_{1}}^{p_{2}}\left(w_{1}^{2}\right)\left(L_{x_{2}}^{q_{2}}\left(w_{2}^{2}\right)\right) \times L_{x_{1}}^{p_{3}}\left(w_{1}^{3}\right)\left(L_{x_{2}}^{q_{3}}\left(w_{2}^{3}\right)\right) \rightarrow L^{r}\left(w_{1} \otimes w_{2}\right)$ for $\frac{1}{p}+\frac{1}{p_{2}}+\frac{1}{p_{3}}=\frac{1}{p}+\frac{1}{q_{2}}+\frac{1}{q_{3}}=\frac{1}{r}$ with $0<r<\infty, 1<p, p_{2}, p_{3}, q_{2}, q_{3}<\infty$, where

$$
\begin{aligned}
& w_{1}\left(x_{1}\right)=\left(w_{1}^{1}\right)^{r / p} \cdot\left(w_{1}^{2}\right)^{r / p_{2}} \cdot\left(w_{1}^{3}\right)^{r / p_{3}} \\
& w_{2}\left(x_{2}\right)=\left(w_{2}^{1}\right)^{r / p} \cdot\left(w_{2}^{2}\right)^{r / q_{2}} \cdot\left(w_{2}^{3}\right)^{r / q_{3}} .
\end{aligned}
$$

In particular, by taking

$$
\begin{aligned}
& w_{1}^{1}=w_{1}^{2}=w_{1}^{3}=w_{1} \in A_{\min \left(p s / 3, p_{2} s / 3, p_{3} s / 3\right),} \\
& w_{2}^{1}=w_{2}^{2}=w_{2}^{3}=w_{2} \in A_{\min \left(p s / 3, q_{2} s / 3, q_{3} s / 3\right)},
\end{aligned}
$$

$T_{3}$ maps $L^{p}\left(w_{1} \otimes w_{2}\right) \times L_{x_{1}}^{p_{2}}\left(w_{1}\right)\left(L_{x_{2}}^{q_{2}}\left(w_{2}\right)\right) \times L_{x_{1}}^{p_{3}}\left(w_{1}\right)\left(L_{x_{2}}^{q_{3}}\left(w_{2}\right)\right) \rightarrow L^{r}\left(w_{1} \otimes w_{2}\right)$,

Proof. As in the proof of Proposition B.3, we decompose the symbol $m$ by using the appropriate cutoff functions, and obtain the corresponding different groups.

We first consider the cases that belong to $I_{1} \otimes I_{2}$, and we take $I_{1}(a) \otimes I_{2}(b)$ for example. Note that by Lemma C.2 $w_{1}\left(x_{1}\right), w_{2}\left(x_{2}\right) \in A_{\infty}$. Then Lemma C.5 implies

$$
\left\|T_{3}(f, g, h)\right\|_{L^{r}\left(w_{1} \otimes w_{2}\right)} \lesssim\left\|\left\{\sum_{j, k}\left|\psi_{1}\left(D / 2^{j}\right) \psi_{2}\left(D / 2^{k}\right) T_{3}(f, g, h)\right|^{2}\right\}^{\frac{1}{2}}\right\|_{L^{r}\left(w_{1} \otimes w_{2}\right)}
$$

Recall we set in Proposition B.3

$$
\begin{aligned}
A_{j, k} & :=\left|\psi_{1}\left(D / 2^{j}\right) \psi_{2}\left(D / 2^{k}\right) T_{3}(f, g, h)\right| \\
& \lesssim\left\|m_{j, k}\right\|_{H^{s, s}}\left(M_{s}\left(\left|\tilde{\psi}_{j}(D) f\right|^{t}\right)\left(x_{1}, x_{2}\right)\right)^{\frac{1}{t}}\left(M_{s}\left(\left|\tilde{\psi}_{k}(D) g\right|^{t}\right)\left(x_{1}, x_{2}\right)\right)^{\frac{1}{t}} \\
& \left(M_{s}\left(|h|^{t}\right)\left(x_{1}, x_{2}\right)\right)^{\frac{1}{t}}
\end{aligned}
$$

where we need $\max (1,3 / s)<t<2$, which now is actually $3 / s<t<2$, since we have assumed $s \leq 3$. Using Hölder's inequality, we have 


$$
\begin{aligned}
& \left\|T_{3}(f, g, h)\right\|_{L^{r}\left(w_{1} \otimes w_{2}\right)} \\
\lesssim & \left\|\left\{\sum_{j, k}\left|\psi_{1}\left(D / 2^{j}\right) \psi_{2}\left(D / 2^{k}\right) T_{3}(f, g, h)\right|^{2}\right\}^{\frac{1}{2}}\right\|_{L^{r}\left(w_{1} \otimes w_{2}\right)} \\
\lesssim & \sup _{j, k}\left\|m_{j, k}\right\|_{H^{s, s}} \|\left[\sum_{j}\left(M_{s}\left(\left|\tilde{\psi}_{j}(D) f\right|^{t}\right)\left(x_{1}, x_{2}\right)\right)^{\frac{2}{t}}\right]^{\frac{1}{2}}\left(M\left(\left|g_{1}\right|^{t}\right)\left(x_{1}\right)\right)^{\frac{1}{t}} \\
\cdot\left[\sum_{k}\left(M\left(\left|\tilde{\psi}_{k}(D) g_{2}\right|^{t}\right)\left(x_{2}\right)\right)^{\frac{2}{t}}\right]^{\frac{1}{2}}\left(M\left(\left|h_{1}\right|^{t}\right)\left(x_{1}\right)\right)^{\frac{1}{t}}\left(M\left(\left|h_{2}\right|^{t}\right)\left(x_{2}\right)\right)^{\frac{1}{t}} \|_{L^{r}\left(w_{1} \otimes w_{2}\right)} & \left\|\left[\sum_{j}\left(M_{s}\left(\left|\tilde{\psi}_{j}(D) f\right|^{t}\right)\left(x_{1}, x_{2}\right)\right)^{\frac{2}{t}}\right]^{\frac{1}{2}}\right\|_{L^{p}\left(w_{1}^{1} \otimes w_{2}^{1}\right)} \\
\lesssim \sup _{j, k}\left\|m_{j, k}\right\|_{H^{s, s}} \| & \left(M\left(\left|g_{1}\right|^{t}\right)\left(x_{1}\right)\right)^{\frac{1}{t}}\left[\sum_{k}\left(M\left(\left|\tilde{\psi}_{k}(D) g_{2}\right|^{t}\right)\left(x_{2}\right)\right)^{\frac{2}{t}}\right]^{\frac{1}{2}}
\end{aligned}
$$

where $1 / p+1 / s_{0}=1 / r$, i.e., $1 / p_{2}+1 / q_{2}=1 / p_{3}+1 / q_{3}=1 / s_{0}$. Then using the tensor product setting, the above can be estimated by

$$
\begin{aligned}
& =\sup _{j, k}\left\|m_{j, k}\right\|_{H^{s, s}}\left\|\left[\sum_{j}\left(M_{s}\left(\left|\tilde{\psi}_{j}(D) f\right|^{t}\right)(x)\right)^{\frac{2}{t}}\right]^{\frac{1}{2}}\right\|_{L^{p}\left(w_{1}^{1} \otimes w_{2}^{1}\right)}
\end{aligned}
$$

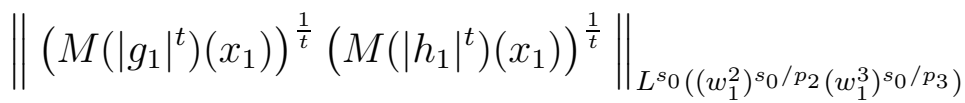

$$
\begin{aligned}
& \left\|\left[\sum_{k}\left(M\left(\left|\tilde{\psi}_{k}(D) g_{2}\right|^{t}\right)\left(x_{2}\right)\right)^{\frac{2}{t}}\right]^{\frac{1}{2}}\left(M\left(\left|h_{2}\right|^{t}\right)\left(x_{2}\right)\right)^{\frac{1}{t}}\right\|_{L^{s_{0}}\left(\left(w_{2}^{2}\right)^{s_{0}} / q_{2}\left(w_{2}^{3}\right)^{s_{0} / q_{3}}\right)} .
\end{aligned}
$$

Again by Hölder's inequality, we have

$$
\begin{aligned}
\lesssim \sup _{j, k}\left\|m_{j, k}\right\|_{H^{s, s}}\left\|\left[\sum_{j}\left(M_{s}\left(\left|\tilde{\psi}_{j}(D) f\right|^{t}\right)(x)\right)^{\frac{2}{t}}\right]^{\frac{1}{2}}\right\|_{L^{p}\left(w_{1}^{1} \otimes w_{2}^{1}\right)} \\
\quad\left\|\left(M\left(\left|g_{1}\right|^{t}\right)\left(x_{1}\right)\right)^{\frac{1}{t}}\right\|_{L^{p_{2}\left(w_{1}^{2}\right)} \|}\left\|\left[\sum_{k}\left(M\left(\left|\tilde{\psi}_{k}(D) g_{2}\right|^{t}\right)\left(x_{2}\right)\right)^{\frac{2}{t}}\right]^{\frac{1}{2}}\right\|_{L^{q_{2}\left(w_{2}^{2}\right)}}
\end{aligned}
$$




$$
\begin{aligned}
& \left\|\left(M\left(\left|h_{1}\right|^{t}\right)\left(x_{1}\right)\right)^{\frac{1}{t}}\right\|_{L^{p_{3}\left(w_{1}^{3}\right)}}\left\|\left(M\left(\left|h_{2}\right|^{t}\right)\left(x_{2}\right)\right)^{\frac{1}{t}}\right\|_{L^{q_{3}\left(w_{2}^{3}\right)}} \\
& =\sup _{j, k}\left\|m_{j, k}\right\|_{H^{s, s}}\left\|\left[\sum_{j}\left(M_{s}\left(\left|\tilde{\psi}_{j}(D) f\right|^{t}\right)(x)\right)^{\frac{2}{t}}\right]^{\frac{t}{2}}\right\|_{L^{p / t}\left(w_{1}^{1} \otimes w_{2}^{1}\right)}^{1 / t} \\
& \left\|M\left(\left|g_{1}\right|^{t}\right)\left(x_{1}\right)\right\|_{L^{p_{2} / t}\left(w_{1}^{2}\right)}^{1 / t}\left\|\left[\sum_{k}\left(M\left(\left|\tilde{\psi}_{k}(D) g_{2}\right|^{t}\right)\left(x_{2}\right)\right)^{\frac{2}{t}}\right]^{\frac{t}{2}}\right\|_{L^{q_{2} / t}\left(w_{2}^{2}\right)}^{1 / t} \\
& \left\|M\left(\left|h_{1}\right|^{t}\right)\left(x_{1}\right)\right\|_{L^{p_{3} / t}\left(w_{1}^{3}\right)}^{1 / t}\left\|M\left(\left|h_{2}\right|^{t}\right)\left(x_{2}\right)\right\|_{L^{q_{3} / t}\left(w_{2}^{3}\right)}^{1 / t} \\
& \lesssim \sup _{j, k}\left\|m_{j, k}\right\|_{H^{s, s}}\|f\|_{L^{p}\left(w_{1}^{1} \otimes w_{2}^{1}\right)}\left\|g_{1}\right\|_{L^{p_{2}\left(w_{1}^{2}\right)}}\left\|g_{2}\right\|_{L^{q_{2}\left(w_{2}^{2}\right)}}\left\|h_{1}\right\|_{L^{p_{3}\left(w_{1}^{3}\right)}}\left\|h_{2}\right\|_{L^{q_{3}\left(w_{2}^{3}\right)}} \text {. }
\end{aligned}
$$

In order to get the last estimate, it needs that $2, p, p_{2}, p_{3}, q_{2}, q_{3}>t$, and

$$
w_{1}^{1} \otimes w_{2}^{1} \in A_{p / t}(\mathbb{R} \times \mathbb{R}), \quad w_{1}^{2} \in A_{p_{2} / t}, \quad w_{1}^{3} \in A_{p_{3} / t}, \quad w_{2}^{2} \in A_{q_{2} / t}, \quad w_{2}^{3} \in A_{q_{3} / t} .
$$

To see why the above can be achieved, first consider the stated assumptions for the weights

$$
\begin{gathered}
\min \left(p, p_{2}, p_{3}, q_{2}, q_{3}\right)>3 / s, 3 / 2<s \leq 3, \quad \text { and } \\
w_{1}^{1}, w_{2}^{1} \in A_{p s / 3}, w_{1}^{2} \in A_{p_{2} s / 3}, w_{1}^{3} \in A_{p_{3} s / 3}, w_{2}^{1} \in A_{p s / 3}, w_{2}^{2} \in A_{q_{2} s / 3}, w_{2}^{3} \in A_{q_{3} s / 3} .
\end{gathered}
$$

The condition (C.5) means it's possible to choose some $t$ with $3 / s<t<\min \left(2, p, p_{2}, p_{3}, q_{2}, q_{3}\right)$, i.e., $1 / \min \left(2, p, p_{2}, p_{3}, q_{2}, q_{3}\right)<1 / t<s / 3$. For condition (C.6), Lemma C.1 implies there exists $\tau_{i}^{j}<s / 3(1 \leq i, j \leq 3)$ such that

$w_{1}^{1} \in A_{p \tau_{1}^{1}}, w_{2}^{1} \in A_{p \tau_{2}^{1}}, w_{1}^{2} \in A_{p_{2} \tau_{1}^{2}}, w_{1}^{3} \in A_{p_{3} \tau_{1}^{3}}, w_{2}^{1} \in A_{p \tau_{2}^{1}}, w_{2}^{2} \in A_{q_{2} \tau_{2}^{2}}, w_{2}^{3} \in A_{q_{3} \tau_{2}^{3}}$.

Now we pick $t$ with $1 / t$ sufficiently close to $s / 3$, such that $\tau_{i}^{j}<1 / t$ for $1 \leq i, j \leq 3$.

Then it follows that

$$
w_{1}^{1}, w_{1}^{2} \in A_{p / t}, \quad w_{1}^{2} \in A_{p_{2} / t}, \quad w_{1}^{3} \in A_{p_{3} / t}, \quad w_{2}^{2} \in A_{q_{2} / t}, \quad w_{2}^{3} \in A_{q_{3} / t},
$$

which implies (C.4).

Using the same argument, we can deal with the cases in other groups. Note that the above verification for condition (C.4) works actually for all the cases, thus we will not repeat it in the rest of the proof. For the operators in $I I_{1} \times I I_{2}$, we consider $I I_{1}(a) \times I I_{2}(b)$ for example. Recall we can write

$$
\begin{aligned}
& \left|T_{3}(f, g, h)\left(x_{1}, x_{2}\right)\right| \\
\lesssim & \sup _{j, k}\left\|m_{j, k}\right\|_{H^{s, s}}\left\{\sum_{j}\left[M_{s}\left(\left|\tilde{\psi}_{j}(D) f\right|^{t}\right)\right]^{\frac{2}{t}}\right\}^{\frac{1}{2}}\left\{\sum_{j, k}\left[M_{s}\left(\left|\tilde{\psi}_{j}(D) \psi_{k}(D) g\right|^{t}\right)\right]^{\frac{2}{t}}\right\}^{\frac{1}{2}}
\end{aligned}
$$




$$
\cdot\left\{\sum_{k}\left[M_{s}\left(\left|\tilde{\psi}_{k}(D) h\right|^{t}\right)\right]^{\frac{2}{t}}\right\}^{\frac{1}{2}},
$$

where the Cauchy-Schwartz inequality is used. Then the Hölder's inequality gives

$$
\begin{aligned}
& \left\|T_{3}(f, g, h)\right\|_{L^{r}\left(w_{1} \otimes w_{2}\right)} \\
& \lesssim \sup _{j, k}\left\|m_{j, k}\right\|_{H^{s, s}}\left\|\left\{\sum_{j}\left[M_{s}\left(\left|\tilde{\psi}_{j}(D) f\right|^{t}\right)\right]^{\frac{2}{t}}\right\}^{\frac{1}{2}}\right\|_{L^{p}\left(w_{1}^{1} \otimes w_{2}^{1}\right)} \|\left\{\sum_{j, k}\left[M_{s}\left(\left|\tilde{\psi}_{j}(D) \psi_{k}(D) g\right|^{t}\right)\right]^{\frac{2}{t}}\right\}^{\frac{1}{2}}
\end{aligned}
$$

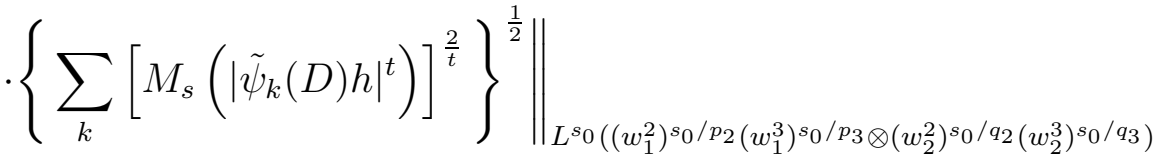

$$
\begin{aligned}
& \lesssim \sup _{j, k}\left\|m_{j, k}\right\|_{H^{s, s}}\left\|\left\{\sum_{j}\left[M\left(\left|\tilde{\psi}_{j}(D) f\right|^{t}\right)\right]^{\frac{2}{t}}\right\}^{\frac{1}{2}}\right\|_{L^{p}\left(w_{1}^{1} \otimes w_{2}^{1}\right)} \\
& \left\|\left\{\sum_{j}\left[M\left(\left|\tilde{\psi}_{j}(D) g_{1}\right|^{t}\right)\right]^{\frac{2}{t}}\right\}^{\frac{1}{2}}\right\|_{L^{p_{2}\left(w_{1}^{2}\right)}}\left\|\left[M\left(\left|h_{1}\right|^{t}\right)\right]^{\frac{1}{t}}\right\|_{L^{p_{3}\left(w_{1}^{3}\right)}} \\
& \left\|\left\{\sum_{k}\left[M\left(\left|\tilde{\psi}_{k}(D) g_{2}\right|^{t}\right)\right]^{\frac{2}{t}}\right\}^{\frac{1}{2}}\right\|_{L^{q_{2}\left(w_{2}^{2}\right)}}\left\|\left\{\sum_{k}\left[M\left(\left|\tilde{\psi}_{k}(D) h_{2}\right|^{t}\right)\right]^{\frac{2}{t}}\right\}^{\frac{1}{2}}\right\|_{L^{q_{3}\left(w_{2}^{3}\right)}} . \\
& \lesssim \sup _{j, k}\left\|m_{j, k}\right\|_{H^{s, s}}\|f\|_{L^{p}\left(w_{1}^{1} \otimes w_{2}^{1}\right)}\left\|g_{1}\right\|_{L^{p_{2}\left(w_{1}^{2}\right)}}\left\|g_{2}\right\|_{L^{q_{2}\left(w_{2}^{2}\right)}}\left\|h_{1}\right\|_{L^{p_{3}\left(w_{1}^{3}\right)}}\left\|h_{2}\right\|_{L^{q_{3}}\left(w_{2}^{3}\right)} \text {. }
\end{aligned}
$$

Then for the situations in $I_{1} \times I I_{2}$. We consider $I_{1}(a) \times I I_{2}(c)$ for an example. Recall

$$
\begin{aligned}
& B_{j}:=\psi_{1}\left(D / 2^{j}\right) T_{3}(f, g, h)\left(x_{1}, x_{2}\right) \\
& \lesssim \sup _{k}\left\|m_{j, k}\right\|_{H^{s, s}}\left\{\sum_{k}\left(M_{s}\left(\left|\tilde{\psi}_{j}(D) \psi_{k}(D) f\right|^{t}\right)\left(x_{1}, x_{2}\right)\right)^{\frac{2}{t}}\right\}^{\frac{1}{2}}\left(M_{s}\left(|g|^{t}\right)\left(x_{1}, x_{2}\right)\right)^{\frac{1}{t}} \\
& \cdot\left\{\sum_{k}\left(M_{s}\left(\left|\tilde{\psi}_{k}(D) h\right|^{t}\right)\left(x_{1}, x_{2}\right)\right)^{\frac{2}{t}}\right\}^{\frac{1}{2}},
\end{aligned}
$$

Then

$$
\begin{aligned}
& \left\|T_{3}(f, g, h)\right\|_{L^{r}\left(w_{1} \otimes w_{2}\right)} \\
\lesssim & \left\|\left\{\sum_{j}\left|\psi_{1}\left(D / 2^{j}\right) T_{3}(f, g, h)\right|^{2}\right\}^{\frac{1}{2}}\right\|_{L^{r}\left(w_{1} \otimes w_{2}\right)}
\end{aligned}
$$




$$
\begin{aligned}
& \lesssim \sup _{j, k}\left\|m_{j, k}\right\|_{H^{s, s}}\left\|\left\{\sum_{j, k}\left(M_{s}\left(\left|\tilde{\psi}_{j}(D) \psi_{k}(D) f\right|^{t}\right)\left(x_{1}, x_{2}\right)\right)^{\frac{2}{t}}\right\}^{\frac{1}{2}}\right\|_{L^{p}\left(w_{1} \otimes w_{2}\right)} \\
& \left\|\left(M\left(\left|g_{1}\right|^{t}\right)\left(x_{1}\right)\right)^{\frac{1}{t}}\right\|_{L^{p_{2}\left(w_{1}^{2}\right)}} \cdot\left\|\left(M\left(\left|h_{1}\right|^{t}\right)\left(x_{1}\right)\right)^{\frac{1}{t}}\right\|_{L^{p_{3}\left(w_{1}^{3}\right)}} \\
& \left\|\left(M\left(\left|g_{2}\right|^{t}\right)\left(x_{2}\right)\right)^{\frac{1}{t}}\right\|_{L^{q_{2}\left(w_{2}^{2}\right)}} \cdot\left\|\left\{\sum_{k}\left(M\left(\left|\tilde{\psi}_{k}(D) h_{2}\right|^{t}\right)\left(x_{2}\right)\right)^{\frac{2}{t}}\right\}^{\frac{1}{2}}\right\|_{L^{q_{3}\left(w_{2}^{3}\right)}} \\
& \lesssim \sup _{j, k}\left\|m_{j, k}\right\|_{H^{s, s}}\|f\|_{L^{p}\left(w_{1} \otimes w_{2}\right)}\left\|g_{1}\right\|_{L^{p_{2}\left(w_{1}^{2}\right)}}\left\|g_{2}\right\|_{L^{q_{2}\left(w_{2}^{2}\right)}}\left\|h_{1}\right\|_{L^{p_{3}\left(w_{1}^{3}\right)}}\left\|h_{2}\right\|_{L^{q_{3}\left(w_{2}^{3}\right)}} .
\end{aligned}
$$

We are now ready to achieve the main goal of this section, namely, establishing the weighted mixed norm estimate for operator (1.7). Since we are less concerned with the limited smoothness of the Hörmander condition of the symbols in the above two propositions, one can simply take $s_{1}=3$ and $s_{2}=2$ in Proposition C.1, and $s=3$ in Proposition C.2, then Theorem 1.9 follows immediately.

\section{REFERENCES}

[1] C.Benea and C. Muscalu, Quasi-Banach valued inequalities via the helicoidal method, J. Funct. Anal. 273 (2017), no. 4, $1295 \mathrm{C} 1353$.

[2] Á. Bényi, D. Maldonado, V. Naibo and H. Torres, On The Hörmander Classes of Bilinear Pseudodifferential Operators, Integr. Equ. Oper. Theory 67 (2010), 341-364.

[3] J. Chen and G. Lu, Hörmander type theorems for multi-linear and multi-parameter Fourier multiplier operators with limited smoothness, Nonlinear Analysis 101 (2014), 98-112.

[4] R. Coifman and Y. Meyer, Au delá des opérateurs pseudo-differentiels. (French) [Beyond pseudodifferential operators] With an English summary. Astérisque, 57. Société Mathématique de France, Paris, 1978.

[5] R. R. Coifman and Y. Meyer, On commutators of singular integrals and bilinear singular integrals, Trans. Amer. Math. Soc. 212 (1975), 315-331.

[6] R. R. Coifman and Y. Meyer, Au-delà des operateurs pseudo-differentiels. Second Edition. Asterisque 57, 1978.

[7] W. Dai and G. Lu, $L^{p}$ estimates for multi-linear and multi-parameter pseudo-differential operators, Bull. Soc. Math. France 143 (2015), no. 3, 567-597. arXiv:1308.4062.

[8] J. Duoandikoetxea, Fourier Analysis, Grad. Stud. Math, vol 29, Amer. Math. Soc, Providence, RI, 2001.

[9] C. Fefferman, E.M. Stein, Some maximal inequalities, Amer. J. Math. 93 (1971), 107-115.

[10] R. Fefferman, E.M. Stein, Singular integrals on product spaces, Adv. Math. 45 (1982) $117 \mathrm{C} 143$.

[11] M. Fujita, N. Tomita, Weighted norm inequalities for multilinear Fourier multipliers, Trans. Amer. Math. Soc. 364 (12) (2012) 6335C6353.

[12] J. Garcĺa-Cuerva, J.L. Rubio de Francia, Weighted Norm Inequalities and Related topics, in: North-Holland Math. Stud., North-Holland, 1985.

[13] L. Grafakos and R. H. Torres, Multilinear Calderón-Zygmund theory, Adv. in Math. 165 (2002),124-164. 
[14] C. Kenig and E. M. Stein, Multilinear estimates and fractional integration, Math. Res. Lett. 6 (1999), 1-15.

[15] G. Lu and L. Zhang, $L^{p}$ estimate for a trilinear pseudo-differential operator with flag symbols, Indiana University Mathematics Journal, 66 (2017), no. 3, 877-900.

[16] A. Miyachi and N. Tomita, Estimates for trilinear flag paraproducts on $L^{\infty}$ and Hardy spaces, Math. Z. (2016) 282: 577-613.

[17] B. Muckenhoupt, Weighted norm inequalities for the Hardy maximal function, Trans. Amer. Math. Soc. 165 (1972) 207C226.

[18] C. Muscalu, Paraproducts with flag singularities I. A case study, Rev. Mat. Iberoam. 23 (2007), no. 2, 705-742.

[19] C. Muscalu, Flag paraproducts, Harmonic analysis and partial differential equations, 131-151, Contemp. Math., 505, Amer. Math. Soc., Providence, RI, 2010.

[20] C. Muscalu and W. Schlag, Classical and Multilinear Harmonic Analysis, II, Cambridge Studies in Advanced Mathematics, vol. 138, Cambridge University Press, Cambridge, 2013.

[21] C. Muscalu, J. Pipher, T. Tao, C. Thiele, Bi-parameter paraproducts, Acta. Math. 193 (2004), 269-296.

[22] C. Muscalu, J. Pipher, T. Tao, C. Thiele, Multi-parameter paraproducts, Rev. Mat. Iberoam. 22 (2006), no. 3, 963-976.

[23] C. Muscalu, Y. Zhai, Five-linear singular integrals of Brascamp-Lieb type, https://arxiv.org/abs/2001.09064

[24] Z. Ruan, Weighted Hardy spaces in the there-parameter case, J. Math. Anal. Appl. 367 (2010) $625 \mathrm{C} 639$.

[25] E. M. Stein, Harmonic Analysis: Real Variable Methods, Orthogonality, and Oscillatory Integrals, Princeton University Press, Princeton (1993).

[26] Y. Zhai, Study of bi-parameter flag paraproducts and bi-parameter stopping-time algorithms., Ph.D. Thesis, Cornell Univ.

Department of Mathematics, University of Connecticut, Storrs, CT 06269

E-mail address, G. Lu: guozhen.lu@uconn.edu

Department of Mathematics, Brown University, Providence, Ri 02912

E-mail address, J. Pipher: jpipher@math.brown.edu

Department of Mathematical Sciences, Binghamton University, Binghamton, Ny 13902

E-mail address, L. Zhang: lzh@math.binghamton.edu 